\title{
A BIOLOGICALLY INSPIRED CMOS IMAGE SENSOR
}





\title{
A BIOLOGICALLY INSPIRED CMOS IMAGE SENSOR
}

\author{
PROEFSCHRIFT
}

ter verkrijging van de graad van doctor aan de Technische Universiteit Delft, op gezag van de Rector Magnificus prof. ir. K.C.A.M. Luyben, voorzitter van het College voor Promoties, in het openbaar te verdedigen op vrijdag 4 februari 2011 om 10.00 uur

door

Mukul SARKAR

Master of Science in Biomedical Engineering University of Technology Aachen, Germany

geboren te Puratan Degree, West Bengal, India. 
Dit proefschrift is goedgekeurd door de promotor:

Prof. dr. ir. A.J.P. Theuwissen

Samenstelling promotiecommissie:

Rector Magnificus

voorzitter

Prof. dr. ir. A.J.P. Theuwissen Technische Universiteit Delft, promotor

Prof. dr. ir. H. Thienpont Vrije Universiteit Brussel, België

Prof. dr. J. Van der Spiegel University of Pennsylvania, USA

Prof. dr. T. Delbruck

ETH Zürich, Schweiz

Prof. dr. ir. C. Van Hoof

KU Leuven/imec, België

Prof. dr. K.A.A. Makinwa

Technische Universiteit Delft

Dr. G. Meynants

CMOSIS, België

Prof. dr. P.J. French

Technische Universiteit Delft, reservelid

Copyright (c) 2011 by M. Sarkar

(Coverpage photo courtesy museum in Germany)

All rights reserved. No part of the material protected by this copyright notice may be reproduced or utilized in any form or by any means, electronic or mechanical, including photocopying, recording or by any information storage and retrieval system, without the prior permission of the author.

ISBN

PRINTED IN NETHERLANDS 
To my family 



\section{Table of contents}

1 Introduction 1

1.1 Perception of vision . . . . . . . . . . . . . . 2

1.2 Challenges and Motivation . . . . . . . . . . 5

1.2.1 Wide field of view . . . . . . . . . . 7

1.2.2 Motion detection . . . . . . . . . . . . . . 7

1.2.3 High sensitivity to low light intensity . . . . . . 8

1.2.4 Polarization . . . . . . . . . . . . . . . 9

1.3 Thesis Organization . . . . . . . . . . . . . . . 10

1.4 References. . . . . . . . . . . . . . . . . 11

\section{Design of a CMOS image sensor for an artificial} $\begin{array}{ll}\text { compound eye } & 15\end{array}$

2.1 Natural compound eye . . . . . . . . . . . . . . . . . . . . . . . . . . . .

2.1 .1 Apposition eye . . . . . . . . . . . . 17

2.1.2 Superposition eye . . . . . . . . . . . . . . 17

2.2 Artificial compound eye . . . . . . . . . . . . . . . . 19

2.3 Micro-optics design . . . . . . . . . . . . . . . . . . . . . . . . . . . . . . . 22

2.4 Photodetector . . . . . . . . . . . . . . . 25

2.5 Sensor overview . . . . . . . . . . . . . . 31

2.5.1 Pixel architecture . . . . . . . . . . . 33 
2.5.2 Pixel operation . . . . . . . . . . . . . . . . 34

2.5.2.1 Image capture phase . . . . . . . . . . . . 34

2.5.2.2 Image storage phase . . . . . . . . . . . 37

2.5.2.3 Image binarization phase . . . . . . . . 40

2.5.2.4 Image readout phase . . . . . . . . . . . . 40

2.5.3 Pixel layout . . . . . . . . . . . . . . . . 41

2.5.4 Row/Column addressing logic . . . . . . . . . . 43

2.5.5 Analog signal chain . . . . . . . . . . . . . . 45

2.5.5.1 Delta differential sampling $(D D S) \ldots \ldots$. . . 46

2.5.5.2 Output amplifier . . . . . . . . . . . . . 49

2.5.6 Digital signal chain . . . . . . . . . . . . . . . . . . 49

2.5.6.1 Digital counter . . . . . . . . . . . 51

2.6 Image sensor characterization . . . . . . . . . . . . 52

2.6 .1 Test setup . . . . . . . . . . . . . . 53

2.6.2 Measurements . . . . . . . . . . . . 55

2.6.2.1 Photo conversion characteristics . . . . . . 55

2.6.2.2 Photon transfer curve PTC . . . . . . . . 56

2.7 Conclusions . . . . . . . . . . . . . . . . . . . . . . . . . . 59

2.8 References . . . . . . . . . . . . . . . . . . . . . 60

3 Design of a CMOS polarization sensor $\quad 63$

3.1 Polarization vision $\ldots \ldots \ldots \ldots$

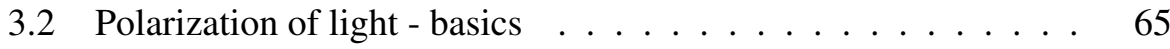

3.2.1 Polarization of light from an unpolarized beam . . . . 67

3.2.1.1 Polarization by absorption . . . . . . . . . . 67

3.2.1.2 Polarization by reflection $\ldots \ldots$. . . . . 68

3.2.1.3 Polarization by refraction . . . . . . . . 68

3.2.1.4 Polarization by scattering . . . . . . . . . . 69

3.2.2 Polarization of light - representation . . . . . . . . . 70 
3.3 Polarization vision in compound eyes . . . . . . . . . 73

3.4 Polarization cameras . . . . . . . . . . . . . . . . . 74

3.5 Wire-grid polarizer . . . . . . . . . . . . 77

3.5.1 Transmittance efficiency and extinction ratio of wiregrid polarizers $\ldots \ldots \ldots \ldots$. . . . . . . 81

3.6 Design of a polarization sensor . . . . . . . . . . . . . 84

3.7 Polarization measurements . . . . . . . . . . . . . . . 86

3.7.1 Measurement setup . . . . . . . . . . . . . . 88

3.7.2 Analog polarization measurements . . . . . . . . . 89

3.8 Wavelength selection using metal grid . . . . . . . . . . . 93

3.9 Conclusions . . . . . . . . . . . . . . . . . . . . . . . 101

3.10 References . . . . . . . . . . . . . . . . . . 101

\section{Material classification using CMOS polarization} sensor

4.1 Introduction . . . . . . . . . . . . . . . . . 106

4.2 Polarization and Fresnel coefficients . . . . . . . . . . . . 107

4.2.1 Polarization properties of a reflected light . . . . . . 109

4.2.2 Fresnel reflectance model . . . . . . . . . . . . . . 114

4.3 Material classification measurements . . . . . . . . . . . . . 116

4.3.1 Measurement setup . . . . . . . . . . . . . . . . 117

4.3.2 Polarization transmittance . . . . . . . . . . . 117

4.3.3 Material classification using the degree of polarization . 120

4.3.4 Material classification using the Stokes parameters _ . . 122

4.3.5 Material classification using polarization Fresnel ratio . 125

4.4 Metal classification . . . . . . . . . . . . . . . . . . . . 128

4.4.1 Metal classification using PFR . . . . . . . . . . 130

4.4.2 Metal classification using degree of polarization . . . . . 131

4.5 Conclusions . . . . . . . . . . . . . . . . . . . . . . . . 134 
4.6 References . . . . . . . . . . . . . . . . . . . . 135

5 Navigation using CMOS polarization sensor $\quad 137$

5.1 Introduction . . . . . . . . . . . . . . . 138

5.2 Celestial compass based on skylight polarization _ . . . . . 141

5.3 Navigation using polarized light by insects . . . . . . . . . . . 145

5.4 Navigation using polarized light for autonomous agents . . . . 148

5.5 Polarization based compass . . . . . . . . . . . . . . . . 151

5.5.1 Measurement setup . . . . . . . . . . . . . . 151

5.5.2 Measurement results . . . . . . . . . . . . . . 151

5.6 Incoming light ray direction detection and sun position detection 156

5.6 .1 Measurement setup . . . . . . . . . . . . . 157

5.6.2 Measurement results for incoming light ray directions . 157

5.6.2.1 Incoming light ray direction detection using ellipticity angle . . . . . . . . . 158

5.6.2.2 Incoming light ray direction detection using azimuthal angle . . . . . . . . . . 159

5.7 Real-time implementation of navigation compass . . . . . . . 164

5.8 Conclusions . . . . . . . . . . . . . . . . . . . . . . . 167

5.9 References. . . . . . . . . . . . . . . . . . . . 168

6 Motion detection and digital polarization $\quad 173$

6.1 Motion detection . . . . . . . . . . . . . . . . . 174

6.1.1 Motion detection - models . . . . . . . . . . . . . 177

6.2 Motion detection - differential imaging . . . . . . . . . . . . 179

6.3 Motion detection - optical flow . . . . . . . . . . . . . . 182

6.3.1 Motion in vertical direction - collision detection . . . . . 184 6.3.1.1 Elementary motion detector (EMD) . . . . . . 184

6.3.1.2 Proposed EMD model for collision detection . 186 
6.3.2 Motion in horizontal direction . . . . . . . . . . . 192

6.4 Illumination invariant and high dynamic range motion detection 194

6.5 Digital polarization . . . . . . . . . . . . . . 201

6.6 Conclusions . . . . . . . . . . . . . . . . . . 203

6.7 Reference . . . . . . . . . . . . . . 205

\section{Conclusions and future work 209}

7.1 Summary of achievements . . . . . . . . . . . . 210

7.1.1 Sensing polarization information . . . . . . . . 210

7.1.2 Using polarization information in material classification 211

7.1.3 Using polarization information in navigation . . . . . 211

7.1.4 Regarding motion/collision detection . . . . . . . 212

7.2 Future works . . . . . . . . . . . . . . . . 213

7.2.1 Multichannel imaging system . . . . . . . . . . . 213

7.2.1.1 Image mapping and distortion correction . . . 214

7.2.2 Wide field of view imaging system with polarization sensitivity . . . . . . . . . . . . . . 218

7.2.3 High angular resolution imaging system with polarization sensitivity . . . . . . . . . . . . . . . 219

7.2.3.1 Real time material classification . . . . . . . 220

7.2.3.2 Real time navigation . . . . . . . . . . . 221

7.2.3.3 Real time sun position detection . . . . . . . 222

7.3 Reference ...................... 223

Summary

Samenvatting 
List of publications

About the author 


\section{1}

\section{Introduction}

Visual perception is the ability to detect light and interpret it. The early explanation of vision was provided by two major ancient Greek schools of thought. One believed in the "emission theory" championed by scholars like Euclid and Ptolemy, according to which vision occurs when light rays emanate from the eyes and intercepted by visual objects. The other school championed by scholars like Aristotle and Galen, believed in "intromission" where vision occurs by something entering the eyes representative of the object [1.1]. The Persian scholar Ibn al-Haytham ('Alhazen') is credited for refining the intromission theory into the modernly accepted theory of perception of vision [1.2]. In his most influential "Book of Optics", he defines vision to be due to the light from objects entering the eye [1.3], [1.4].

Perception is unavoidably selective; one cannot see all there is to see. Visual perception is not merely a translation of retinal stimuli. As John Berger and Jean Mohr [1.5] quote "A photograph quotes from appearances, but in quoting, simplifies them". The primary objective of this thesis is to simplify the perceived visual world (i.e. the photograph) for machine vision applications.

This chapter presents a short overview of various perceptions of vision prevalent in different optical vision systems. The motivation and challenges to design an optical vision system based on the compound eye of the insects as opposed to the more conventional approach of mimicking single aperture eyes is presented. 


\subsection{Perception of vision}

Objects become visible through many different phenomena of light example reflection, refraction. The way in which creatures see differs in regard to their shape, perception, color visualization, resolution and depth perception. Most animals see the world in fuzzy shades of gray while some animals can see in total darkness, or even see colors beyond the human visual spectrum. Hunting birds use binocular vision to spot prey from thousands of feet above. Dogs and cats are color blind but have better peripheral and night vision than humans and are more sensitive to movements. The sensitivity of owls is 50-1000 times greater to low light intensities than unaided human night vision. Snakes have thermal pits in addition to normal eyes extending their spectral sensitivity into the infrared. Horses and zebras have their eyes pointing sideways giving them outstanding peripheral vision thus warning them of the presence of predators.

Eyes can be simply defined as organs or visual systems for spatial vision. There are fundamentally two different ways in which high resolution spatial vision can be achieved: either by increasing the number of photoreceptors in the visual system or by multiplying the visual system in its entirety [1.6]. These two alternatives lead to simple or single chambered eyes and compound eyes, respectively. The human eye is a single chambered eye with a lens and works much like a camera as shown in figure 1.1(a). Animals with such eyes comprise less than $6 \%$ of the species in the animal kingdom. More than $77 \%$ of the known animal species are insects and crustaceans with compound eyes as shown in figure 1.1(b). Such compound or complex eyes are composed of numerous simple single aperture eyes.

The performance of any eye is principally affected by three structural and two environmental features [1.7]. Among the former are (a) the angular spacing of the receptors, which determines the spatial resolution; (b) the quality of the optical structures; (c) the diameter of the photoreceptors. Among the latter are (a) the amount of light available to the receptors, and (b) motion (self or image) [1.8].

The term "resolution" is used in a loose way to mean "ability to resolve fine details" [1.8]. Resolving power or visual acuity is used to describe the smallest single object that an eye can detect. The two fundamental limitations to the resolving power or resolution of eyes are the wave (diffraction) and the particle (photon noise) nature of light. The wide lens aperture in humans provides a very fine resolution due to the narrow airy disc of a point object caused by diffraction. An airy disc is a diffraction pattern resulting from a uniformly illuminated circular aperture which has a bright region in the center with concentric rings 


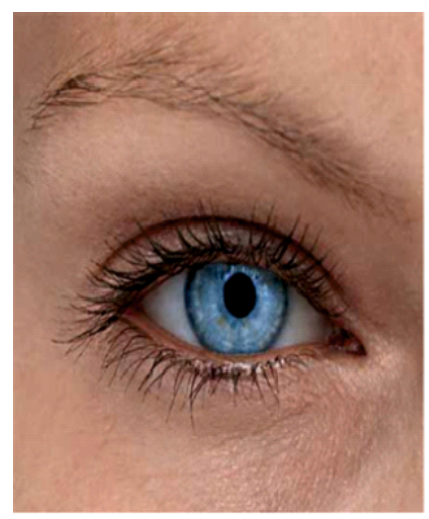

\section{Lens eye}

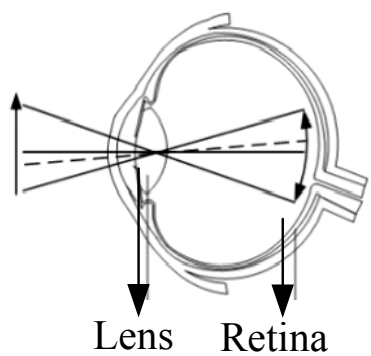

(a)
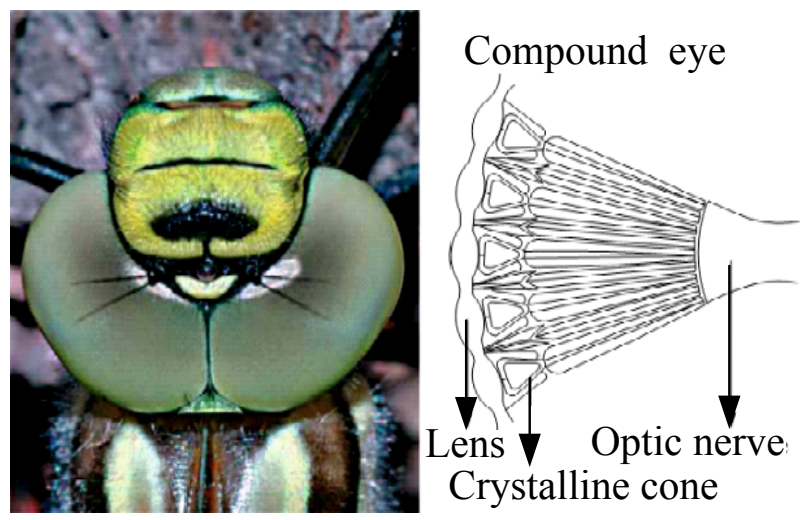

(b)

Figure 1.1: (a) Single aperture eye, (b) Compound eye [1.9].

around. The resolution of the small individual lenses of the compound eye is diffraction limited to about $1^{\circ}$.

The other limitation of the resolution is the photon noise. Each photoreceptor can only detect a finite number of quanta, and any such count is associated with an uncertainty. Effectively, this limits the ability to detect differences in luminance (contrasts) across the image. For a given size, an eye can split up the captured light between many receptor cells (high spatial resolution), or it can use the light to activate fewer receptor cells more strongly (high sensitivity). The luminance generated by natural light sources varies over eight orders of magnitude between sunlight and starlight [1.7]. In 1941, Hecht et al. [1.10] 
discovered that human vision is limited in dim light by the small numbers of available photons. At the absolute human threshold, each receptor receives one photon on an average for every 40 minutes and this is the same for insects [1.10]. The uncertainty in the detection of the number of quanta (photons), increases in low light conditions, thus degrading the visual signal-to-noise ratio [1.11], [1.12]. As there needs to be a certain amount of light captured in order to be reliably detected, the eyes of nocturnal animals tend to be optimized for sensitivity rather than spatial resolution [1.8]. The visual sensitivity of the compound eye is enhanced by the summation of the spatial information in the neighboring visual channels [1.13], [1.14], [1.15].

The visual acuity depends on both the spatial and the temporal resolution of the eye, as visual systems often detect and respond to objects that move relative to the animal itself [1.16]. As an object moves across the visual field, the individual receptor channels are progressively turned on and off due to a series of changes in the light intensity. This creates a "flicker-effect" known as the flicker frequency rate. The temporal resolving power for moving processes of a compound eye is considerably higher than for a single aperture eye, because of the high flicker frequency fusion rate. With their high speed of reaction, flying insects can resolve 250 images per second. In contrast the human eye sees only about 24 images per second. Eyes with high flicker fusion frequencies are able to recover responsiveness in shorter times than those with lower fusion frequency. Thus compound eyes are ideal for motion detection.

Motion blur is the smearing of a moving visual image due to the finite width of the impulse response of the photoreceptor. The combination of low spatial and high temporal resolutions makes the insects' visual system less sensitive to motion blur than the human visual system. Theoretically, a fly's vision becomes blurred at an angular velocity of about $1.5^{\circ} / 7 \mathrm{~ms}=200^{\circ} / \mathrm{s}$, while in humans this occurs at $2^{\prime} / 25 \mathrm{~ms}=1.4^{\circ} / \mathrm{s}$. The wide field of view of the compound eye also aids in motion detection. The field of view of a compound eye is as close to $360^{\circ}$ with respect to a two-dimensional plane, whereas the field of view of human eyes is around $160-208^{\circ}$.

Although an image of sorts forms in the eye, it is now accepted that for the majority of insects an image per se has no physiological significance. Figure 1.2 shows artistic impression of two images, as perceived by a housefly. The real function of the compound eye appears to be that of movement perception.

Besides being excellent in motion detection, compound eyes are also able to detect polarization. Light emanating from the sky and reflected light from a watery surface or shiny leaves is polarized i.e. it has greater vibration in some 

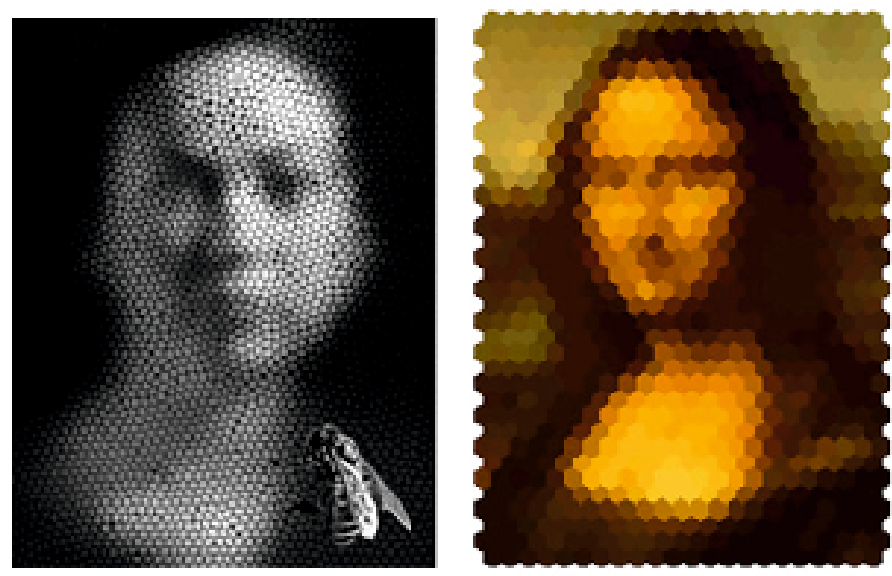

Figure 1.2: An artistic impression of Insects (housefly's) vision [1.17].

planes than in others. The microvillar organization of the insect's photoreceptors makes its visual systems inherently sensitive to the plane of polarization of light.

\subsection{Challenges and Motivation}

The natural evolution of eyes to increase the spatial resolution by either increasing the number of photoreceptors in the visual system or by multiplying the visual system in its entirety has been of interest in the image sensor research field over the last 25 years. The increase of photoreceptors can be compared to the present day phenomenon of the "mega-pixel-race" and the design of autonomous multiple visual systems can be related loosely to the present day "neuromorphic image sensors". The neuromorphic approach implements specialized sensory processing function inspired by biological systems in analog electronic circuits. Neuromorphic vision sensors process images directly at the focal plane with circuits that try to emulate the first stages of visual processing in biological systems.

The perceptual world of animals is very different from that of humans as explained in section 1.1. Insects in general are exquisitely sensitive to image motion, which provides them with useful cues for avoiding obstacles and distance based landing. Thus insects' eyes are better suited for compact, robust and cheap vision systems.

The photoreceptor in a visual system is used to capture and convert the light photons to electric charge. The two most common semiconductor based 
photoreceptors used for artificial vision systems are CCD (charge coupled device) and CMOS (Complementary Metal Oxide Semiconductor) image sensors. CCDs produce a uniform output increasing the image quality, as all the pixels are only devoted to capture light. However CCDs usually have very limited output nodes, often only one for the pixel's charge to be converted into voltage to be sent offchip. Also, CCDs have to be read out completely, and thus are inconvenient for region based readouts, affecting the output bandwidth of the sensor. They also require supporting circuits which increase the complexity and size of the camera design, thereby increasing the cost and power consumption. CMOS image sensors on the other hand use generic fabrication process, and in principle work very similarly to CCD sensors. In addition to being less expensive when compared to CCDs, they also allow for the integration of processing circuitry with the photoreceptor. CMOS image sensors also allow for random accessibility, enhancing the output bandwidth. The image quality of a CMOS image sensor is however not at par with that of a CCD as the in-pixel and in-column circuitry often produces a "fixed pattern noise" in the image.

Over the last decade, the resolution of the image sensor has been constantly increasing. Digital cameras with 12 Megapixels or more are now available in the market. To maintain the image quality with increasing resolution or decreasing pixel size, the hardware and firmware performance has to be increased, significantly affecting the complexity, size, cost and power consumption of the system.

Another area of research in vision systems has focused on neuromorphic or intelligent ways of processing the visual data. Until recently the artificial eye has been modeled based on the single aperture eye of the humans, wherein the output of the video-camera is scanned by a variety of spatial and temporal filters. This needs processing of huge volumes of data, defeating the purpose of having simple low power high sensitive vision sensors. Insects use minimal computations even for complicated functions like vision guided flying and thus are more suited for machine vision applications than single aperture eyes.

As we have discussed in the previous section, the compound eyes of insects offer low spatial resolution compared to single aperture eye but also offer other advantages for machine vision applications. These include:

- Wide field of view

- Better suited to detect moving object

- Higher sensitivity to light intensity

- Ability to detect polarization information

The focus of the thesis is to study each of the above mentioned advantages and to investigate new concepts and design techniques to implement the advantages 
of the compound eye on conventional CMOS image sensors. Each of these advantages will be addressed in detail in this thesis.

\subsubsection{Wide field of view}

A wide field of view is required in many applications like endoscopy, surveillance and automobile cameras. The most popular method to obtain this is image mosaicing, wherein multiple images are 'stitched' to obtain a larger image. This has a great redundancy in data, with each point being captured multiple times.

In comparison to the human eye which uses a spherical volume, compound eyes use only a spherical shell, permitting a large field of view but requiring less signal processing. Compound eyes have a field of view of near $360^{\circ}$ obtained by the superposition of multiple simple lenses. Two such artificial insect facet eyes with this ability are the cluster eye and the artificial apposition compound eye (APCO) [1.18]. The cluster eye is inspired by the apposition eye and consists of three micro-lens array of different pitch. The optical axis of these micro-lenses are titled with respect to each other, and each micro-lens samples a small part of the object. The number of micro-lenses used determines the sensitivity and resolution. The $A P C O$ system is also based on the apposition eye where each channel samples one direction in space. The APCO system consists of a microlens array and a pinhole array with a slightly different pitch than the micro-lens array. The pinhole array defines the sensitivity and resolution. Both cluster eye and the APCO system has lower field of view $(F O V)$ because the lenses are not tilted outwards, as is the case in insects.

The major technical drawback with wide angular field of view in artificial compound eyes is that the spherical optical world is mapped onto flat surfaces of the photo detector. The curved base of natural insect eyes offers several advantages including panoramic vision. Panoramic vision would mean far more information with which to monitor and control one's movement in the world. From a technological point of view, special fabrication techniques are being developed to have curved photodetectors, which would aid in the wide field of view vision [1.19].

\subsubsection{Motion detection}

In conventional machine vision, motion is detected using a CMOS or CCD image sensor which samples the visual field at tens to thousands of times per second. This generates a huge volume of data to be processed for recognition and 
localization of the target. The precision of the system has a positive correlation with the computation payload. The higher the precision required, the higher is the payload.

The high flicker fusion frequency of the compound eye allows for much faster processing of the images than the single chambered eyes. The reaction time reported for honeybees to an object suddenly appearing in its visual field is $0.01 \mathrm{~s}$ while that for humans is $0.05 \mathrm{~s}$. Additionally the division of the scene into multiple sub-images allows parallel image processing and non sequential readout of the selected sub-images. This facilitates high speed object tracking while reducing image processing bottlenecks.

There are numerous architectures for motion detection based on the compound eye. Optical flow measurements have been explored by [1.20], [1.21], while frame differencing is used in [1.22]. Multi-resolution architecture has been proposed leading to variable spatial acuity imaging or region based motion detection [1.23], [1.24]. A CMOS image sensor with integrated circuits for basic decision making and image compression on the focal plane has also been presented [1.25]. The correlation motion detectors have been used to explain the direction selective motion using elementary motion detectors (EMD) [1.26]. One of the objectives of this thesis is to investigate on the algorithms to design a fast and simple motion detection algorithm with minimal complexity and processing.

\subsubsection{High sensitivity to low light intensity}

A real-world scene is composed of varying levels of brightness within it. The human eye has a very high dynamic range that enables it to detect subtle variation in the brightness and interpret scenes under different illumination conditions. However, while human vision is very poor in low light conditions some insects are known to have very good night vision. In dim light, to increase the visual signal-to-noise ratio, the visual system has to collect as much light as possible. The receptive field is optically enhanced in insects by summing the outputs of neighboring visual channels (spatial summation) or by increasing the sampling time also known as exposure period (temporal summation). The spatiotemporal summation can extend vision to light intensities more than 100,000 times dimmer than when relied on optics alone [1.27]. In apposition eyes, wider facets and wider photoreceptors can increase the sensitivity by one to two log units [1.28]. Receptor integration time may increase up to five fold in the dark. The enhancement of visual sensitivity by summation has negative effects on the spatial and temporal resolution. 
As vision based safety measures for automotives eventually become mandatory in Europe starting 2013 [1.29], imaging innovations in low light vision systems will have to be in place. The current area of CMOS image sensors in the automotive industry focuses on high dynamic range and near infra-red type of image sensors. The dynamic range of an artificial optical system can be enhanced either by changes in the exposure period or with an adaptive photodetector [1.30]. These sensors coupled with night vision ability and more signal processing for intelligent decisions within the image sensor would be a value addition in assisted driving.

\subsubsection{Polarization}

The three basic characteristics of light are intensity, color and polarization. Polarization provides a more general description of light than either the intensity or the color alone, and can therefore provide richer sets of descriptive physical constraints for the interpretation of the imaged scene. Further a polarization sensitive image sensor along with the ability to measure intensity and color will make it possible to sense the complete set of electromagnetic parameters of light incident on the camera.

The presence of linearly polarized light (the most common type of polarization in nature) in the optical environment can be qualitatively demonstrated by the use of a linear polarizer. The latest polarization image sensors utilize optical imaging systems that are external to the detectors [1.31]. These polarization filters are either single or multi-axis arrays which measure the polarization information in real time. The compactness of design and the speed at which polarized images are generated can be enhanced greatly by incorporating an array of microscopic polarization filtering optics directly onto a photosensitive chip. Micro-polarizers can be made either from organic materials [1.32] or using metallic wire grid available with the standard CMOS technology [1.33].

Light rays get reflected when they strike a reflecting surface, and the reflected light is polarized depending on the incident light and the nature of the reflecting surface. This polarized component can be used to detect the nature of the surface, for example to discriminate between a metal and a dielectric surface [1.34].

The polarized light in the optical environment is also known to be used by insects such as desert ants for navigation. The ability of the desert ant to navigate effortlessly in complex environments has been a subject of research in robotics [1.35]. The current vision sensors for navigation use a generalized algorithm of capturing two-dimensional images using a standard CMOS or a 
CCD camera and then processing those captured images for vector calculations to determine the motion and direction vector. This involves intensive processing along with high power consumption, as most of the post processing of the images is done in the digital domain on a FPGA or a microcontroller. Some of the cheap computational strategies that insects use to navigate have been already modeled in the past [1.36], [1.37].

In conclusion, the focus of this thesis can be summarized as

- Design of an image sensor with an intelligent or a smart pixel, to extend the functionality of the active pixel. The presence of a large number of active elements will limit the resolution. However as a large number of images can be processed at the pixel level, the overall output bandwidth of the image sensor will be improved. Furthermore pixel level processing will also reduce the amount of digital computations helping to design low power fast motion detectors.

- Design of a polarization detection sensor using a metallic wire grid using the metal layers available with a standard CMOS technology. By spatially orienting the micro-polarizer in varying directions polarization information from the optical world can be obtained. Further it is desired to show the real time detection and analysis of polarization information in machine vision applications like material classification and navigation.

\subsection{Thesis Organization}

This thesis consists of seven chapters. Chapter two begins with an overview of the compound eye, following which a description of the artificial compound eye designed in collaboration with the Vrije Universiteit Brussel (VUB) is presented. The design and operation of the designed image sensor along with the performance characterization are also described.

Chapter three begins with an overview of the basic theory behind the polarization of light and its states. The absorption of the incident electromagnetic waves using specifically aligned metallic grid layers and its effect on the transmission intensity and wavelength are discussed using theoretical simulations. The micropolarizer realized in the standard CMOS process and placed on top of the photodiode with varying transmission axis is described and characterized. The polarization information detected using the wire grid micro-polarizer can be used for many applications. Two such applications, material classification and navigation, are presented in chapter four and five respectively. 
Chapter four begins with the overview of the Fresnel reflections of a polarized light ray after specular reflection. The metallic wire grid micro-polarizer in the designed image sensor allows computing parallel and perpendicular Fresnel reflection coefficients along with the maximum and the minimum transmitted irradiance after reflection from the material surface. Using these measured parameters, various methods described in the literature are used to test the ability of the polarized reflected light to distinguish between a metallic and a dielectric surface. The measured coefficients are further shown to allow for differentiating between various metallic surfaces based on conductivity.

Chapter five begins with the introduction to the natural navigation patterns as exhibited by insects like Cataglyphis fortis. Navigation using the polarized state of the incoming light ray is proposed. The azimuthal and the elliptical position of the incoming light rays can be detected using the polarized state of the incoming light ray. This can further be used to detect the position of the sun, as naturally available skylight is always polarized and the degree of polarization depends on the position of the sun.

Chapter six presents the ability of the image sensor to detect motion based on optical flow and differential imaging. The one-dimensional binary optical flow is used to detect collision (vertical motion) and also horizontal motion. The dynamic range of a sensor plays an important role in detecting motion. Partial charge transfer mechanism is shown to increase the dynamic range and also shows invariance to the changes in the background illumination. The onedimensional optical flow varies with the polarization angle and can be used to present polarization in digital form.

Chapter seven presents the final conclusions of the thesis along with suggestions for further research that can be pursued.

\subsection{References}

[1.1] http://en.wikipedia.org/wiki/Visual_perception.

[1.2] A. Sabra, The Optics of Ibn al-Haytham. London: Warburg Institute, 1989. ISBN: 0854810722.

[1.3] A. Sabra, "Ibn al-haytham, brief life of an arab mathematician: died circa 1040," Harvard Magazine, pp. 54-55, 2003.

[1.4] A. Sabra, "Ibn al-haytham's criticisms of ptolemy's optics," Journal of the History of Philolosophy, vol. 4, pp. 145-149, 1966.

[1.5] J. Berger and J. Mohr, Another Way of Telling. New York: Pantheon, 1982. 
[1.6] D. Nilsson, "The evolution of eyes and visually guided behaviour," Philosophical Transactions of the Royal Society London B: Biological Sciences, vol. 364, no. 1531, pp. 2833-2847, 2009.

[1.7] M. Land and D. Nilsson, Animal eyes. Oxford, UK: Oxford University Press, 2002. ISBN: 0198575645.

[1.8] M. Land, "Visual acuity in insects," Annual Review of Entomology, vol. 42, no. 1, pp. 147-177, 1977.

[1.9] "Innovation," Carl Zeiss Magazine, no. 17, pp. 16-17, 2006.

[1.10] M. Pirenne, Vision and the Eye. London: Chapman and Hall, 1967.

[1.11] H. de Vries, "The quantum character of light and its bearing upon threshold of vision, the differential sensitivity and visual acuity of the eye," Physica, vol. 10, pp. 553-564, 1943.

[1.12] A. Rose, "The relative sensitivities of television pickup tubes, photographic film, and the human eye," Proceedings of the Institute of Radio Engineering, vol. 30, no. 6, pp. 293-300, 1942.

[1.13] M. Srinivasan, S. Laughlin, and A. Dubs, "Predictive coding: A fresh view of inhibition in the retina," Philosophical Transactions of the Royal Society London B: Biological Sciences, vol. 216, no. 1205, pp. 427-459, 1982.

[1.14] Y. Tsukamoto, R. Smith, and P. Sterling, "Collective coding of correlated cone signals in the retinal ganglion cell," Proceedings of the National Academy of Sciences America, vol. 87, pp. 1860-1864, 1990.

[1.15] J. van Hateren, "A theory of maximizing sensory information," Biological Cybernetics, vol. 68, no. 1, pp. 23-29, 1992.

[1.16] M. Srinivasan and G. Bernard, "The effect of motion on visual acuity of the compound eye: a theoretical analysis," Vision Research, vol. 15, pp. 515-525, 1975.

[1.17] www.admin.cam.ac.uk/news/press/dpp/2000061501.

[1.18] J. Duparre, M. Eisener, and K. Weible, "Miniaturized imaging systems," Microelectronic Engineering, vol. 67-68, no. 1, pp. 461-472, 2003.

[1.19] H. C. Ko, M. Stoykovic, J. Song, V. Malyarchuk, W. Choi, C. Yu, J. G. III, J. Xiao, S. Wang, Y. Huang, and J. Rogers, "A hemispherical electronic eye camera based on compressible silicon optoelectronics," Nature, vol. 454, pp. 748-753, 2008. 
[1.20] A. Moini, A. Bouzerdoum, K. Eshraghian, A. Yakovleff, X. Nguyen, A. Blanksby, R. Beare, D. Abbott, and R. Bogner, "An insect vision-based motion detection chip," Journal of Solid-State Circuits, vol. 32, no. 2, pp. 279-284, 1997.

[1.21] S. Mehta and R. Cummings, "Normal optical flow measurement on a CMOS APS imager," Proceedings of International Symposium on Circuits and Systems, vol. 4, pp. 848-851, 2004.

[1.22] V. Milirud, L. Fleshel, W. Zhang, G. Jullien, and O. Pecht, "A wide dynamic range CMOS active pixel sensor with frame difference," Proceedings of International Symposium on Circuits and Systems, vol. 1, pp. 588-591, 2005.

[1.23] S. Kemeny, R. Panicacci, B. Pain, L. Matthies, and E. Fossum, "Multiresolution image sensor," IEEE Transactions on Circuits and Systems for Video Technology, vol. 7, no. 4, pp. 575-583, 1997.

[1.24] F. Saffih and R. Hornsey, "Multiresolution CMOS image sensor," Technical Digest of SPIE Opto-Canada, pp. 425-428, 2002.

[1.25] U. Mallik, M. Clapp, E. Choi, G. Cauwenberghs, and R. Cummings, "Temporal change threshold detection imager," IEEE International SolidState Circuits Conference, Digest of Technical Papers, vol. 1, pp. 362-363, 2005.

[1.26] A. Borst, "Correlation versus gradient type motion detectors: the pros and cons," Philosophical Transactions of the Royal Society London B: Biological Sciences, vol. 362, no. 1479, pp. 369-374, 2007.

[1.27] E. Warrant, "Seeing better at night:life style, eye design and the optimum strategy of spatial and temporal summation," Vision Research, vol. 39, no. 9, pp. 1611-1630, 1999.

[1.28] D. Williams, "Changes of photoreceptor performance associated with the daily turnover of photoreceptor membrane in locusts," Journal of Comparative Physiology A: Sensory, Neural, and Behavioral Physiology, vol. 150, pp. 509-519, 1983.

[1.29] L. Robin and J. Baron, "CMOS image sensors technologies and market 2010 report," tech. rep., Market research reports, Yole dveloppement, 2010.

[1.30] A. Darmont, "Methods to extend the dynamic range of snapshot active pixels sensors," Proceedings of SPIE, International Society of Optical Engineering, vol. 6816, pp. 681603.1-681603.11, 2008. 
[1.31] A. Andreou and Z. Kalayjian, "Polarization imaging: principles and integrated polarimeters," IEEE Sensors Journal, vol. 2, no. 6, pp. 566-576, 2002.

[1.32] X. Zhao, F. Boussaid, A. Bermak, and V. Chigrinov, "Thin photopatterned micropolarizer array for CMOS image sensors," IEEE Photonics Technology Letters, vol. 21, no. 12, pp. 805-807, 2009.

[1.33] T. Tokuda, H. Yamada, K. Sasagawa, and J. Ohta, "Polarization analyzing CMOS image sensor with monolithically embedded polarizer for microchemistry systems," IEEE Transactions on Biomedical Circuits and Systems, vol. 3, no. 5, pp. 259-266, 209.

[1.34] L. Wolff, "Polarization based material classification from specular reflection," IEEE Transactions on Pattern Analysis and Machine Intelligence, vol. 12, no. 11, pp. 1059-1071, 1990.

[1.35] R. Wehner, B. Michel, and P. Antonsen, "Visual navigation in insects: coupling of egocentric and geocentric information," Journal of Experimental Biology, vol. 199, pp. 129-140, 1996.

[1.36] D. Lambrinos, M. Maris, H. Kobayashi, T. Labhart, R. Pfeifer, and R. Wehner, "An autonomous agent navigating with a polarized light compass," Adaptive Behaviour, vol. 6, no. 1, pp. 131-161, 1997.

[1.37] K. Usher, P. Ridley, and P. Corke, "A camera as a polarized light compass: preliminary experiments," Proceedings of Australian Conference on Robotics and Automation, pp. 116-120, 2001. 


\section{2}

\section{Design of a CMOS image sensor for an artificial compound eye}

The compound eye is one of the most compact vision systems found in nature. The eye is a compound of individual lenses, each one with their own photoreceptor arrays. This visual system allows an insect to fly with a limited intelligence and brain processing power. In comparison to single-aperture eyes, compound eyes have much wider field of view $(F O V)$, better capability to detect moving objects, higher sensitivity to light intensity, but much lower spatial resolution.

Section 2.1 of this chapter discusses the nature and types of natural compound eye of insects. Section 2.2 gives an overview of the design necessities of an artificial compound eye. The two main design elements of an artificial compound eye are the micro-optical lens and the photodetector array. The micro-optical lens is briefly described in section 2.3 . Section 2.4 describes the design of a $128 \times 128$ pixel CMOS image sensor chip with in-pixel analog memory and a 1-bit dynamic memory in a standard 0.18 $\mu \mathrm{m}$ 1-poly-3-metal CMOS CIS (CMOS Image Sensor) process. The characterization and measurement results of the image sensor are also presented in section 2.6. 


\subsection{Natural compound eye}

The compound eye of an insect is a faceted structure consisting of individual, radially-arranged optical channel units called ommatidia. Each ommatidium captures light from a certain cone of angle of incidence contributing a spot to the visual image, which consists of the total inputs from all the ommatidia in the eye resulting in a mosaic like picture of the world. At the micro level, compound eyes' individual viewing channels on curved lenses have minimal aberrations, or focusing errors that cause blurring.

Each ommatidium as shown in figure 2.1 consists of a lens that forms an image onto the tip of the light sensitive visual cells rhabdom, a transparent crystalline cone to guide the light and absorptive pigments between the ommatidium [2.1]. The light sensitive parts of the visual cells are microvillis, which are an array of tubelike membranes containing the visual pigment rhodopsin. The visual pigments (rhabdomeres) are highly refractive materials, and are ideal for collection the available light with minimal losses as the available light keeps getting totally internally reflected. This visual information is transduced into electrical signals by the photoreceptor cells at the level of the retina. This transduced signal from the retina is processed by three layers of the visual ganglia: the lamina, the medulla, and the lobula complex.

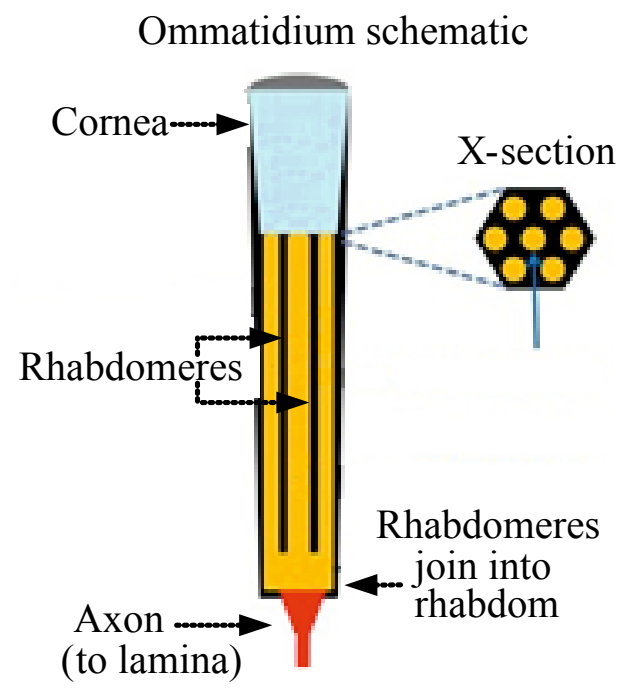

Figure 2.1: Schematic of an ommatidium [2.2]. 
Compound eyes can broadly be classified into two types: apposition and superposition types of compound eyes [2.3].

\subsubsection{Apposition eye}

In an apposition eye, each optical channel has its own receptor. The rhabdomeres and cones touch each other and there exists an absorptive screening pigment between the ommatidia. This reduces the amount of light that can reach a rhabdom from cones other than the one above it, thus keeping the angle of acceptance of light of each ommatidium low. In such eyes each optic unit represents only one sample point. Apposition eyes are generally found in dayactive insects such as ants, wasps, dragonflies, bees and cockroaches.

\section{Apposition compound eye}

Lens Ommatidia

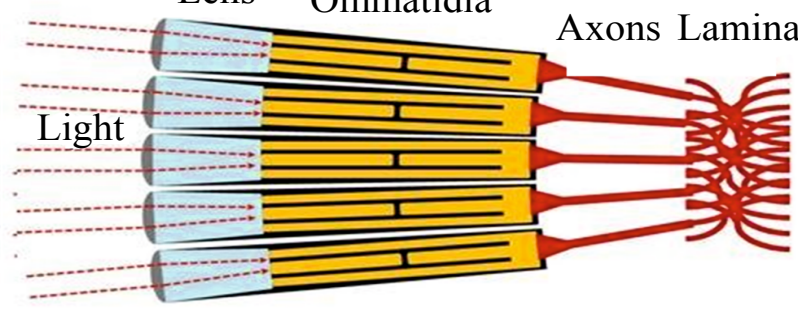

Figure 2.2: Apposition eye [2.2].

\subsubsection{Superposition eye}

In a superposition compound eye the light from different adjacent channels is guided to a common receptor. The superposition eyes are typically more sensitive to light, and this type is found mainly in animals that live under low light conditions, such as moths and crustaceans living in dim mid-water regions of the ocean. Superposition compound eyes can be subdivided into three types: refractive, reflective and neural as shown in figure 2.3. Refractive superposition eyes have an open space between the lens array and the rhabdom (array of photoreceptors). In reflective superposition eyes, the lenses are actually mirrors that reflect the light from a certain direction to a location on the rhabdom. In a neural superposition eye neural circuits puts together the signals from the individual small eye facets. 

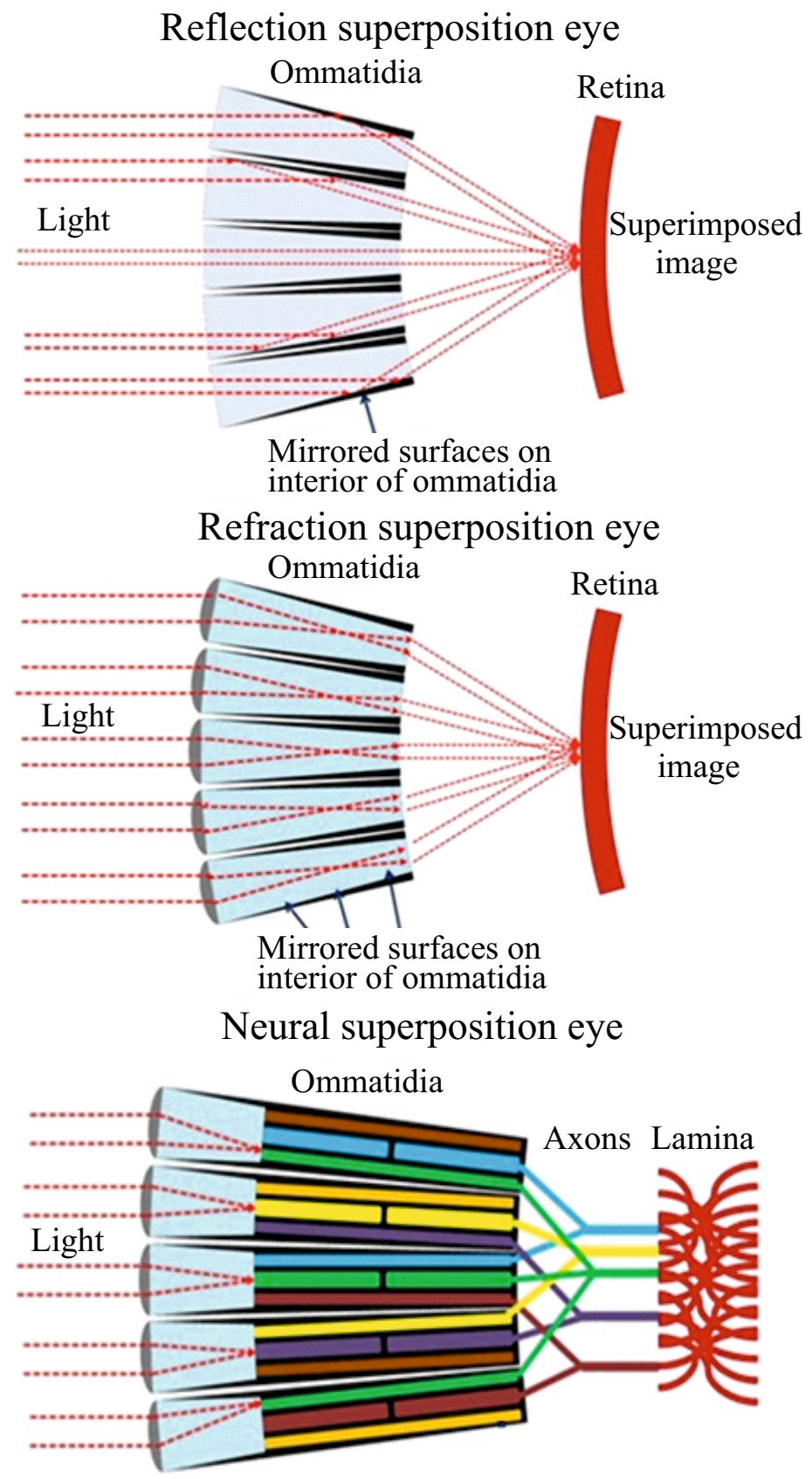

Figure 2.3: Superposition eyes: reflection, refraction and neural type [2.2]. 


\subsection{Artificial compound eye}

Early research in machine vision was aimed at developing systems for general purpose vision. The algorithms implemented were aimed at extracting as much information as possible from a single scene, or from a small number of scenes captured at fixed locations. Over the last few years the research has focused more towards active vision systems where there is an interaction between the viewing device and the external world. Traditionally, problems in machine vision have been tackled through reasoning that is based primarily on geometry, mathematics and logic, with little consideration of how these problems might be solved by natural visual systems. This approach produces solutions that are often computationally complex, sensitive to noise, and sometimes too general purpose to be really effective. There is a growing trend towards exploring strategies for artificial vision that take advantage of the biological systems wherein there is more interaction between the viewing device and the external world.

The artificial compound eye has a number of advantages over ordinary single chamber optical system. Since it is a single sensor with multiple lenses each eyelet lens contributes its own sub-image from different directions to a large fieldof-view, which facilitates sub-imaging and parallel image processing.

Research on artificial compound eyes falls into two categories: (a) multiple camera systems wherein the scene is electronically distributed across multiple lenses using multiple detector arrays or (b) single camera system wherein the scene is optically distributed across multiple lenses using a single detector array. Most of the compound eye literature falls into the first category, although the second category has been catching up in the last few years [2.4].

The model of an artificial visual system based on the single camera system with multiple lenses/aperture to transfer the image through separated optical channels [2.5] is shown in figure 2.4(a). The artificial apposition compound eye consists of a micro-lens array positioned on a substrate, preferably with optical isolation of the channels, and an optoelectronic detector array of different pitch in the micro-lenses' focal plane. The pitch difference enables the different viewing directions of each optical channel. Each channel's optical axis points in a different direction with the optical axes of the channels directed outward if the pitch of the receptor array is smaller than that of the micro-lens array.

A major difference between the insect facet eyes and the artificial eyes is the shape of the detector which is flat in the artificial case and curved for the insect eyes. The arrangement of the microlens layer and the detector layer in a natural compound eye is shown in figure 2.4(b). Each optical channel focuses 


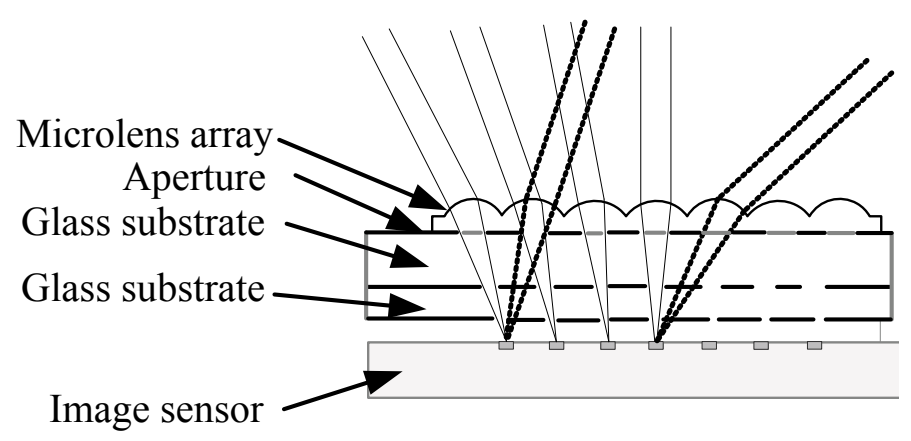

(a)

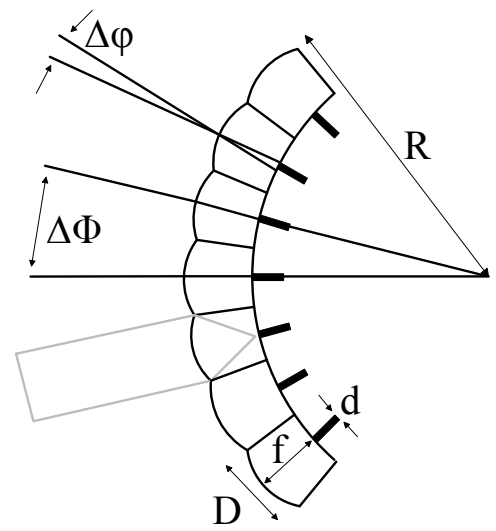

(b)

Figure 2.4: ((a) Structure of an artificial compound eye (b) structure of a natural compound eye.

the light coming from the object points lying on the channels' optical axis. Due to this bending, each ommatidium points towards a different angular direction. Therefore these compound eyes exhibit a very large field of view (FOV) while the single channels are working on-axis and are not suffering from off-axis aberrations [2.6]. The field of view of an eye is defined as the angle in object space, over which objects are observable by the eye. From a technological point of view it is more difficult to work with a curved CMOS detector than with a flat one, although progress has been made in this field [2.7].

A theoretical model of a multichannel or multiple apertures imaging system 
similar to the compound eye of insects is shown in figure 2.5. In the multiple lens system each lens, helps to form a sub image of the object seen with a different angle in the field of view.

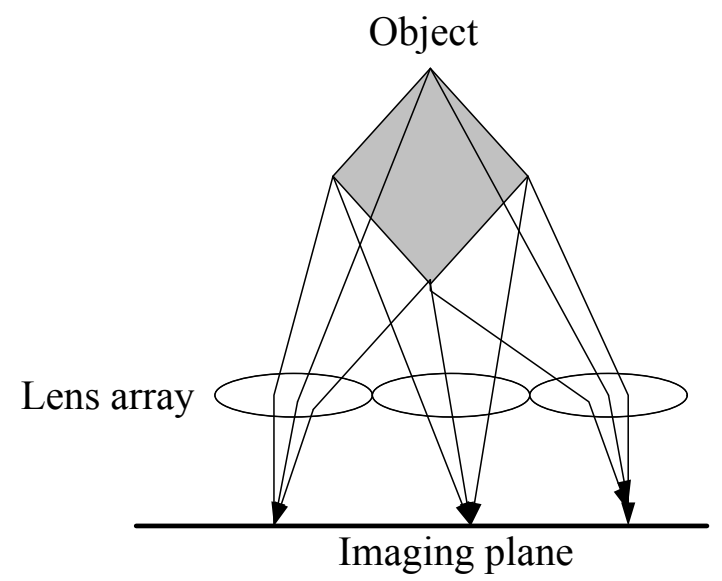

Figure 2.5: The multiple lenses capture each pyramid of the same object.

If each lens in the lens array has $n$ sub-pixels along one dimension then the spatial resolution of the system is reduced by a factor of $n$, producing low resolution sub-images at each lens [2.8]. This photographic technique has already been described as "Integral Photography" (because the resulting integral image is the sum of individual image) by G. Lipmann and H. E. Ives. Many possible implementation of such a multiple lens imaging system can be found in literature today [2.8], [2.9], [2.10]. Due to the reduced imaging field for each micro-lens in multichannel imaging, the requirements on the micro-lenses are relatively moderate, although high resolution is still required. Implementation of the multichannel imaging system can be significantly cheaper than the conventional approach of using multiple single-axis cameras. The problems of multichannel imaging are related to crosstalk and a limited interconnection distance.

The two major design elements of an artificial optical visual system as shown in figure 2.5 are the micro-lens array and photoreceptors or the image sensor. As stated in chapter 1 , the performance of the eyes depend on the angular spacing of the receptors, which determines how finely an image can be resolved. The interommatidial angle in insects is typically $1^{\circ}(0.0175 \mathrm{rad})$ and thus an angular resolution of near $1^{\circ}$ is desired for the micro-lens array. The angular resolution is defined as the smallest angular difference between two incident beams which results in non-overlapping point spread functions $(P S F s)$. PSF is defined as the distribution of the relative intensity over relative distance. Usually for every 
$F O V$, diffraction is more important than geometrical abberations, however optical simulations showed that for a $F O V$ of near $25^{\circ}$, geometrical abberation becomes more important than the diffraction. Thus for $F O V$ greater then $25^{\circ}$, the angular resolution worsens. To have the desired angular resolution of $1^{\circ}$, individual micro-lens with a FOV of $25^{\circ}$ was designed. An array of such individual microlenses was used to enhance the total field of view of the micro-optical system. The design of the micro-lens array is discussed in section 2.3.

Due to robustness and cost effectiveness we chose to work with a flat CMOS detector. From the optical simulations, it was found that in flat facets (detectors), due to the diffraction issues reducing the facet dimensions below $25 \mu \mathrm{m}$ doesn't help to obtain a better focusing. Furthermore the dimensions should not be smaller than $10 \mu \mathrm{m}$. In curved facets however a better focussing is obtained for facets larger than $25 \mu \mathrm{m}$. To utilize the entire area of $5 \mathrm{~mm} \times 5 \mathrm{~mm}$ available in a multiple wafer run $(M P W)$ from Europractice, an imaging array of $128 \times 128$ was selected with a pixel pitch of $25 \mu \mathrm{m}$ and a photodiode area of $10 \mu \mathrm{m} \times 10 \mu \mathrm{m}$. The designed image sensor is described in detail in section 2.5.

\subsection{Micro-optics design}

In this section, the micro-optical lens designed by Els Moens from the TONA group of Vrije Universiteit Brussel (VUB) as part of a collaborative project is described briefly. The system uses a micro-lens array to form multiple images, which are captured on a photo-detector array. This emulates the compound eyes of insects. The goal is to design a multichannel imaging system with a wide field of view, in this case near $124^{\circ}$ with an angular resolution of less than $1^{\circ}$ and also to find a good trade-off between the image resolution and sensitivity. A brief overview of the micro-lens design is presented in this section.

The technological problem of mapping the spherical world onto the planar photodetector surface is solved by dividing the detector plane into 25 zones and an array of $5 \times 5$ micro-lenses covering all the zones. Each zone has a total FOV of near $25^{\circ}$, and capture the light from certain angles with a resolution of $1^{\circ}$, while there are $25 \times 25$ pixels under each micro-lens.

Figure 2.6 shows the 25 separate channels designed to map the spherical word onto the planar surface. Each point in the far field of the image sensor can be described by two spherical coordinates: the angles phi $(\varphi)$ or azimuthal angle and theta $(\theta)$ or the elevation angle. The CMOS detector is divided into 25 equal zones and each zone maps a circle with radius equivalent to the elevation angle $\varphi$. A quarter of the total $F O V$, is separated into 9 channels by varying $\theta$ in three 
steps for $\varphi=25^{\circ}$ and in five steps for $\varphi=50^{\circ}$ as shown in figure 2.6.

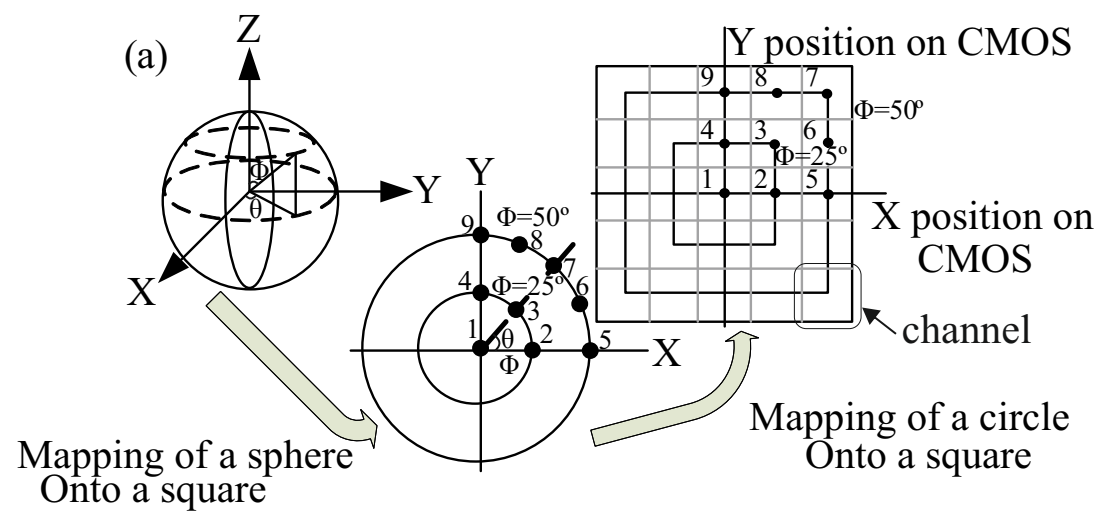

(b)

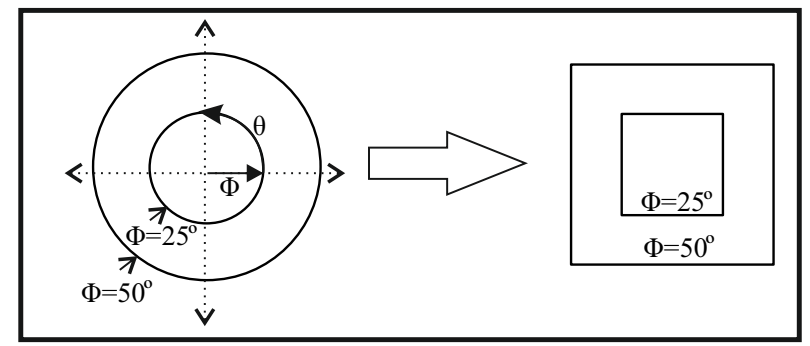

Figure 2.6: Angle to position transformation: (a) Mapping of a sphere onto a square. (b) Angles phi $(\varphi)$ and theta $(\theta)$ for the center directions of angles of incidence for the first nine channels.

To sample a wide field of view (FOV) of $124^{\circ}$, an overlap of the different angular regions mapped by the separate optical channels is allowed. Figure 2.7 shows a quarter of this total $F O V$. It is very difficult to avoid overlap when we want to cover the complete $F O V$ due to the problem of mapping a spherical angular distribution to a square detector.

The microlens array can either have one lens for one angle, where each lens samples one direction in space or one lens for more angle, where each lens samples more directions in space. Real insect eyes use one or more lenses to sample the light from one direction in space, the focus is still good as the curved facet allows the light to be focused on the center of the detector. For flat facet, this can be achieved by tilting the zones to improve focussing, but fabrication is too difficult and the detection plane should be tilted too. Thus in order to achieve the desired $1^{\circ}$ angular resolution, a two lens structure is used as shown 


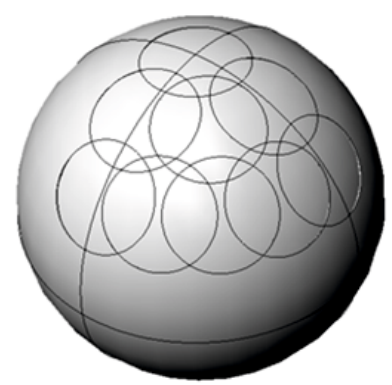

Figure 2.7: Overlap of the different sub-FOVs.

in figure 2.8(a). The function of the first lens is to capture the correct part of light from the total FOV and to have some focusing towards the second lens. The second lens focuses the light towards a spot on the detector. The scanning electron microscopy (SEM) photographs of the top and bottom lenses of the optical channels are shown in the figure 2.8(b), (c). The lenses, substrates and pedestals are fabricated in poly-methyl-methacrylate (

Figure 2.9 shows the simulated light spots on the detector. The central spot and the outer spots originating from the incident rays for which the channel was optimized, are shown. The other spots come from incident rays with an angular difference of $1^{\circ}$. It is clear that all the spots are distinguishable on the detector.

A quantitative merit function for the image quality is the contrast of the image, represented by the modulation transfer function $(M T F)$. The simulated MTF for the described lens array (channels 1-9, as shown in figure 2.6) is shown in figure 2.10.

A maximum contrast of 0.3 line pairs per degree $\left(\mathrm{LP} /{ }^{\circ}\right)$ and an angular resolution of $1^{\circ}$ (down to $0.5^{\circ}$ for some channels) with a total $F O V$ of $124^{\circ}$ have been presented [2.11]. This system is comparable in resolution to others found in literature, however the FOV is much better [2.4], [2.5].

As discussed, to sample the wide field of view, an overlap of the different angular regions mapped by the separate optical channels is allowed. This means that certain directions will be sampled by more than one pixel of the CMOS detector. However these distorted images can be corrected because there is a direct relationship between the angle of incidence and position on the detector. It was not possible to test the micro-lens array with the photodetector array, due to the delay in fabrication of micro-lens array, however the image distortion correction algorithms are described in detail in section 7.2 to be carried out as a future work. 


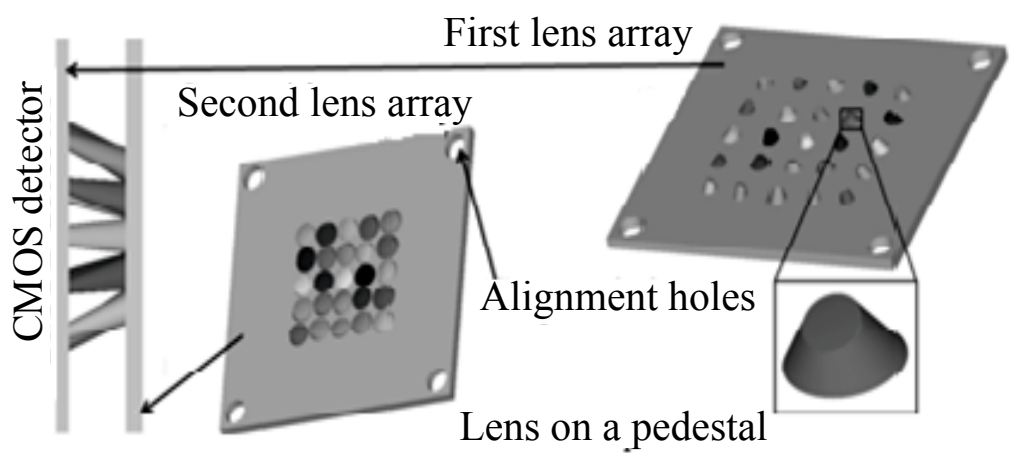

(a)

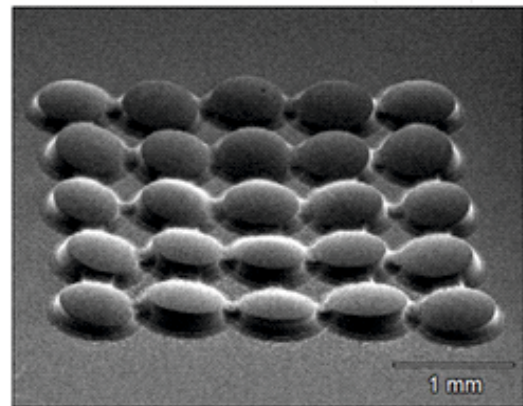

(b)

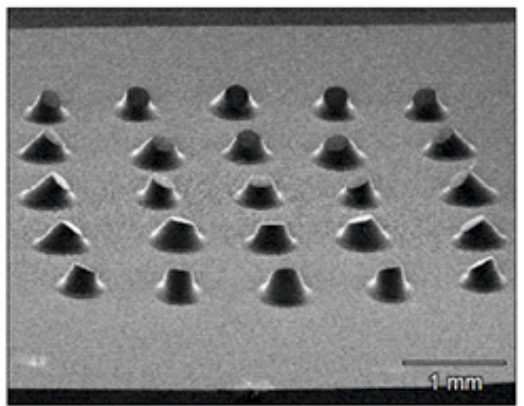

(c)

Figure 2.8: (a) Cross-section of the two lens structure, first lens array, capturing the total FOV, second lens array focusing the light on to the detector with $1^{\circ}$ angular resolution (b) SEM photograph of the first lens array (c) SEM photograph of the second lens array [2.11].

\subsection{Photodetector}

The photoelectric effect is the basic physical process of light detection in solid state photodetectors. The incident electromagnetic wave (light) is converted into an electrical signal by releasing and accelerating current-conducting carriers within the semiconductor [2.12]. In semiconductor such as silicon, there are few free electrons thus the valence band is completely filled and the conduction band is empty, and these two bands are separated by an energy gap. In order to generate an electrical signal, electrons have to be excited from the valence band into the conduction band.

When light is incident on a semiconductor, it may be reflected, absorbed or transmitted. The energy of the absorbed light particles (photons) forces the 


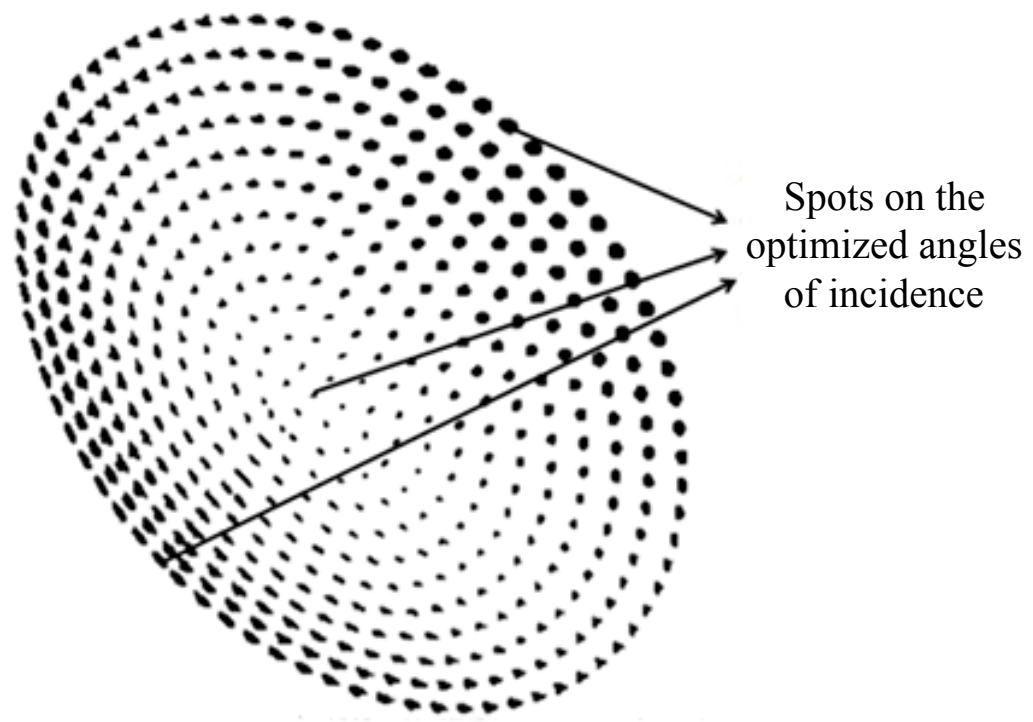

Figure 2.9: Channel seven: Light spots on the detector. There is an angular resolution of $1^{\circ}$.

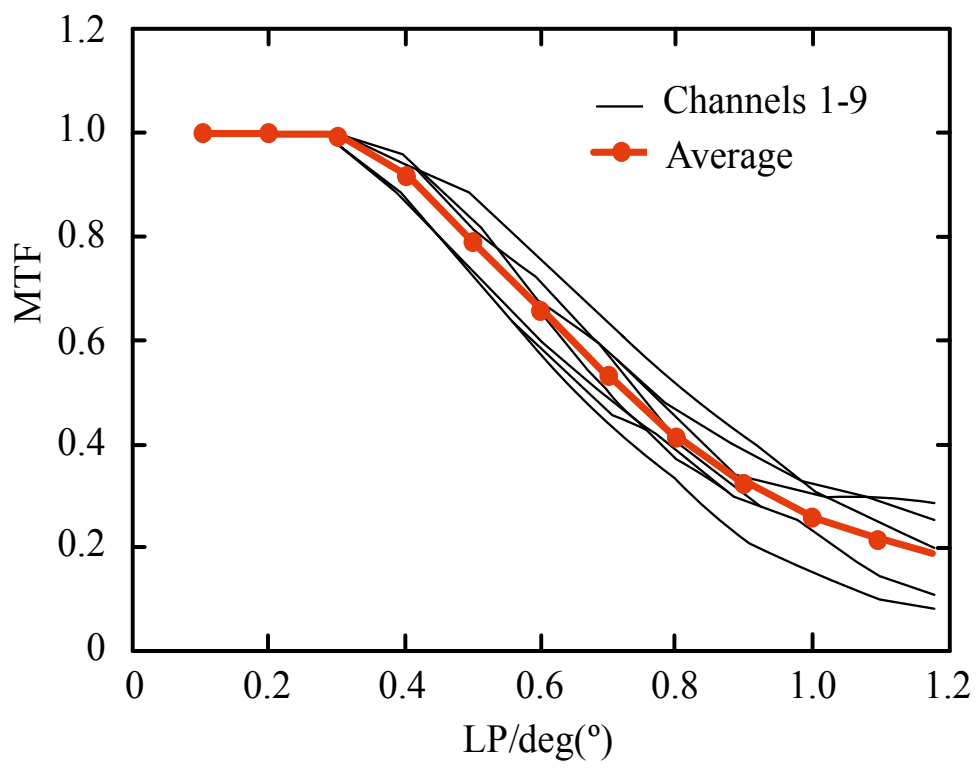

Figure 2.10: MTF for the different channels of the double tilted lens design. 
electrons to excite from the valence band into the conduction band when the absorbed energy is higher than the energy gap, thus producing electron-hole pairs. In silicon, the photon energy should be $\geq 1.119 \mathrm{eV}$ at $300^{\circ} \mathrm{K}$, which corresponds to a light wavelength of $\lambda=1050 \mathrm{~nm}$. The number of electrons excited thermally from the valence band into the conduction band in silicon is given by:

$$
n_{i}=2\left(\frac{2 \pi m_{e} k T}{h^{2}}\right)^{3 / 2} \exp \left(\frac{E_{g}}{2 k T}\right)\left(\approx 10^{10} / \mathrm{cm}^{3} \text { at } T=300^{\circ} \mathrm{K}\right)
$$

where $n_{i}$ is the number of thermally excited electrons, $h$ is the Plank's constant, $k$ is Boltzmann's constant, $T$ is the absolute temperature, $E_{g}$ is the band gap energy and $m_{e}$ is the effective mass of the electron.

Silicon based photodiodes are sensitive to light in the wide spectral range of 200-1200nm, which extends from deep ultraviolet through the visible to the near infrared. The most popular types of semiconductor based photodetectors used in image sensors are the reverse-biased $p-n$ junction photodiode and the $p^{+} / n / p$ pinned photodiode. Though several other photosensitive structures are possible in a standard $n$-well CMOS process, here the discussion will be limited to $p-n$ and $p^{+} / n / p$ junction photodiodes.

A $p$ - $n$ junction is formed when an $n$-type semiconductor is placed in contact with a $p$-type semiconductor. Since the $n$-type region has excess of electrons, the concentration gradient causes the electrons to diffuse from the $n$-type region to $p$-type region and the holes diffuse from the $p$-type to the $n$-type region. This diffusion process is continued till equilibrium is reached when the electric field of these carriers, is equal to the concentration gradient thus forming a junction. In order to function as a photodiode, the $p-n$ junction is reverse biased. This forms a depletion region between the $p$-type region and the $n$-type region which is depleted of mobile charges. Light absorbed in the depletion region generates electron-hole pairs, which contribute to the generation of the photocurrent as shown in figure 2.11 .

The photodiode acts as a capacitor with the boundaries of the depletion region as the two plates of a parallel plate capacitor. At any applied reverse bias voltage $V$ the capacitance for a step junction is given by:

$$
C=\frac{K_{s} \epsilon_{0}}{W_{d}} A_{j}=K_{s} \epsilon_{0} A_{j}\left[2 K_{s} \epsilon_{0} P \mu\left(V+V_{b i}\right)\right]^{-1 / 2}
$$

where $W_{d}$ is the depletion region width, $A_{j}$ is the diffused area, $K_{s}$ the dielectric constant, $\epsilon_{0}$ the permittivity of free space, $P$ the resistivity of the substrate, $\mu$ the 


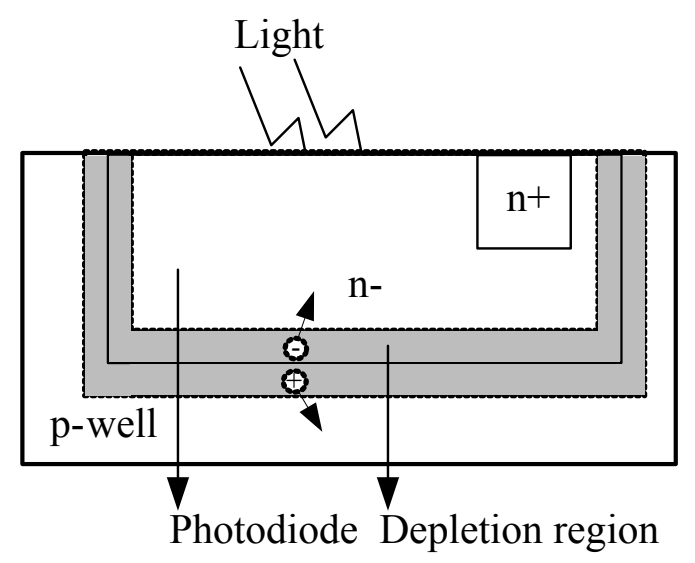

Figure 2.11: n-well/p-well (sub) photodiode.

mobility of the majority carriers in the substrate, $V_{b i}$ is the built-in voltage and $V$ is the applied voltage across the junction.

Photodiodes are usually operated in photoconductive mode, where, a reverse voltage is applied to the diode and the resulting photocurrent is measured. The dependence of the photocurrent on the light power can be very linear over six or more orders of magnitude.

The photocurrent of an ordinary photodiode is very small (in the range of femto- to picoamperes) under typical illumination conditions. Thus, a charge integration based operation is common in many active pixel sensors [2.13]. The basic idea of direct integration is illustrated in figure2.12(a).

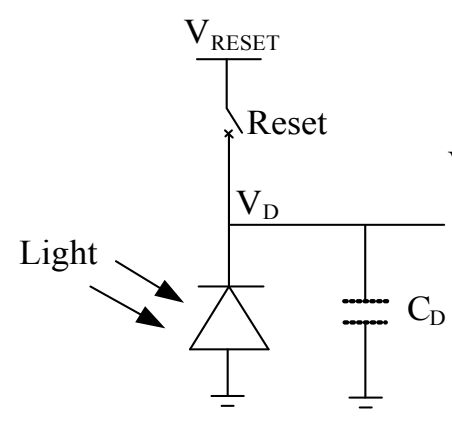

(a)

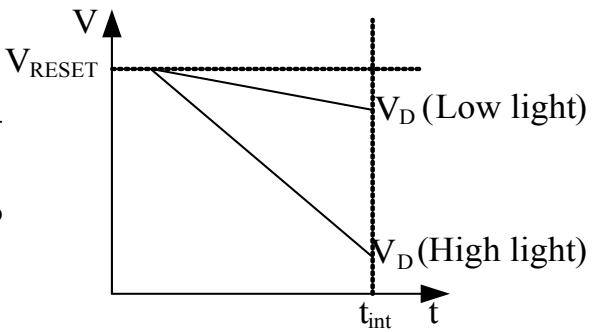

(b)

Figure 2.12: (a) Pixel in integration mode of operation (b) charge versus time for two photocurrent value. 
The photodiode is first reset to a voltage $V_{R E S E T}\left(V_{D}=V_{R E S E T}\right)$. The reset switch is then opened (disconnected) and the diode capacitance $C_{D}$ is discharged by the photocurrent. This capacitance consists of the capacitance of any connected device at the node $V$ plus the photodiode junction capacitance itself. We can write:

$$
C(V) \frac{d V(t)}{d t}=-i_{\text {photo }}-i_{d a r k}
$$

where $i_{\text {photo }}$ is the photogenerated current and $i_{\text {dark }}$ is the current generated in dark.

The photocurrent is directly proportional to the illumination levels, thus the resulting voltage drop across the photodiode capacitance $C_{D}$ is proportional to the light intensity and the integration time. Thus to measure the light intensity, the change in the voltage across the photodiode after a certain integration time $\left(t_{i n t}\right)$ is measured. Examples of the voltage versus time characteristics for low light and high light conditions are shown in the figure 2.12(b) [2.14].

The pinned photodiode $p^{+} / n / p$ provides an improved photoresponsivity compared to standard $p-n$ photodiode [2.15] due to its low dark current and good blue response. The concept of pinned photodiodes is borrowed from surface pinned interline CCD structures. It needs additional wafer implantation steps and an additional transistor (transfer gate) compared to the standard $p-n$ photodiodes to improve noise performance, particularly reset noise, to reduce image lag and to suppress the dark currents [2.16]. The additional transistor allows extended functionalities such as electronic shuttering and dynamic range extension [2.17]. The cross section of a pinned photodiode is shown in the figure 2.13, showing also the transfer gate $(T G)$.

In a pinned photodiode the $n^{-}$terminal is separated from the surface by a $p^{+}$ pinning layer. The $p^{+}$pinning layer is pinned to the substrate voltage. The $p^{+} / n$ well photodiodes where the $p^{+}$in enclosed within its own $n$-well is not used as pinned photodiode. The $p^{+} / n-w e l l$ photodiodes are not suitable for dense arrays because of the huge separation required between two wells. The passivation of defect and surface states at the $\mathrm{Si} / \mathrm{SiO}_{2}$ interface in the pinned photodiodes contributes to the lowering of the thermal noise and dark currents as explained later. The transfer gate separates the floating diffusion node from the photodiode. This allows for the optimization of the floating diffusion node capacitance by sizing the $T G$ transistor. The transfer gate is an asymmetric device which couples the photodiode and the floating diffusion node.

The pinning voltage for the $p^{+}$pinning layer is $1 \mathrm{~V}$ for the selected technology. 


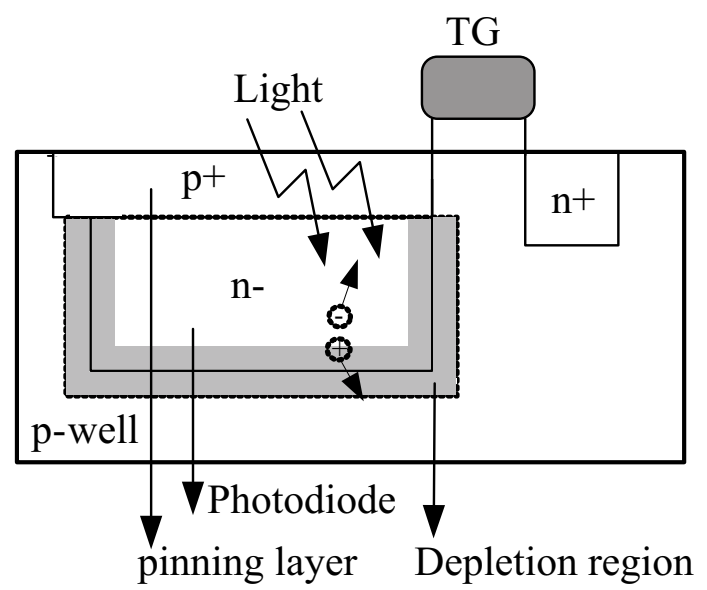

Figure 2.13: $\left(p^{+} / n\right.$-well/p-well(sub) photodiode.

When the photodiode is reset to the pinning voltage, a full depletion of the photodiode is achieved. In the full depletion conditions the depletion regions of $p^{+}-n^{-}$and $n^{-}-p$ meet, eliminating the majority carrier in the $n$ region. A full depletion condition of the pinned photodiodes is preferred, to suppress dark currents and to reduce the $k T C$ noise component due to the mobile electrons left after reset. If the pinned photodiode is not fully depleted, the mobile electrons left would contribute to additional $k T C$ noise after reset. However since the pinning voltage of the photodiode is fixed, the charge transfer efficiency from the photodiode to the floating diffusion node is low which lowers the saturation charge or well capacity of the pinned photodiodes. Well capacity is defined as the maximum amount of charge (in electrons) that can be held by the integration capacitance of the photodiode. The well capacity of a pinned photodiode can be represented by $Q_{P D}=\left(V_{\text {pinning }}-V_{\text {blooming }}\right) C_{P D}$, where $V_{\text {pinning }}$ is the pinning voltage, $V_{\text {blooming }}$ is the blooming voltage of the pixel and $C_{P D}$ is the capacitance of the photodiode. The reduction of $V_{\text {blooming }}$ is limited by the minimum voltage required for stopping the photo-generated electrons from overflowing into the silicon substrate during high illuminations. Therefore, to increase the well capacity of the pixel, $V_{\text {pinning }}$ or $C_{P D}$ should be increased. However, it is very difficult to increase the charge storage capacitance as the pixel size shrinks and a fixed $V_{\text {pinning }}$ results in a low well capacity. At the end of the exposure time, photogenerated carriers are accumulated and transferred to the floating diffusion $n^{+}(F D)$ by opening the transfer gate $T G$. 


\subsection{Sensor overview}

The pinned photodiode described in section 2.4 is used to design a prototype image sensor using $0.18 \mu \mathrm{m} 3$-metal-1-poly CIS process from UMC. The sensor architecture of the designed image sensor is shown in figure 2.14.

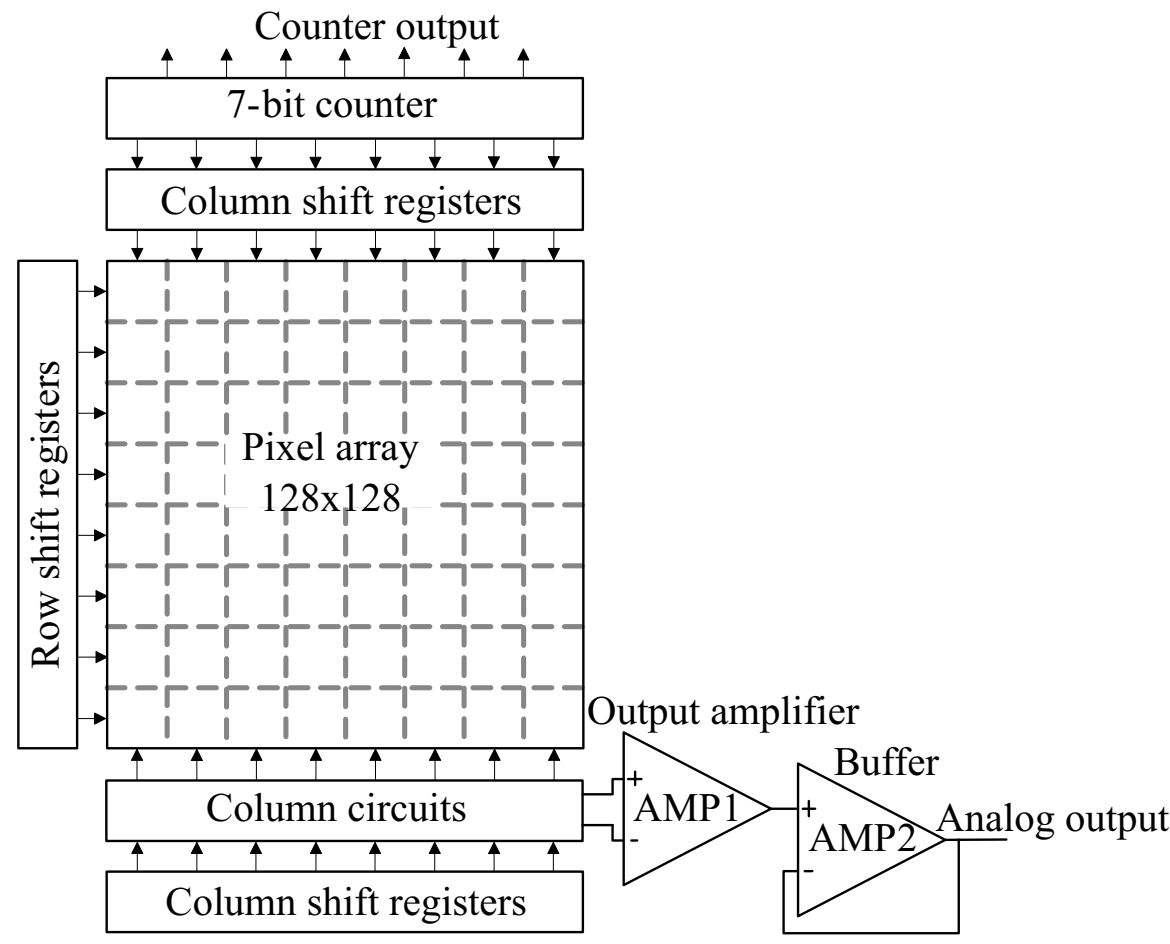

Figure 2.14: Sensor architecture.

The chip is divided into four main blocks:

1. the pixel array,

2. the analog readout circuit,

3. the digital readout circuit and

4. the row select logic plus timing control block.

The pixel array is located at the center, with the photodiodes and their associated circuitry for analog and digital computations. It contains an array of 128 by 128 pixels. Each pixel contains a pinned photodiode and 33 transistors including $T G$ to perform low level image processing. The size of the pixel is 
$25 \mu \mathrm{m} \times 25 \mu \mathrm{m}$ and the size of the photodiode is $10 \mu \mathrm{m} \times 10 \mu \mathrm{m}$ which corresponds to a $16 \%$ pixel fill factor. One half of the pixel array of the designed image sensor is used to analyze polarization information using metallic wire grid structures explained in chapter 3 . The other half is available for normal imaging applications.

The analog readout circuit is placed below the pixel array. It consists of column-wise double differential sampling circuits, an output amplifier, a buffer and a column shift register. The analog output provides the analog voltage at each pixel.

The digital readout circuit is placed at the top and consists of a 7-bit counter and a column shift register. The 7-bit counter is used to count the number of active high pixels in each row.

Finally, the left side is dedicated to the row select logic and timing control blocks to address each row of pixels sequentially.

Table 2.1 summarizes the specifications of the sensor.

\begin{tabular}{|l|l|}
\hline Process & $0.18 \mu \mathrm{m} 1 \mathrm{P} 3 \mathrm{M}$ UMC CIS process \\
\hline Active imager size & $3.2 \mathrm{~mm}(\mathrm{H}) \times 3.2 \mathrm{~mm}(\mathrm{~V})$ \\
\hline Chip Size & $4 \mathrm{~mm}(\mathrm{H}) \times 5 \mathrm{~mm}(\mathrm{~V})$ \\
\hline Active pixels & $128 \times 128$ \\
\hline Pixel size & $25 \mu \mathrm{m} \times 25 \mu \mathrm{m}$ \\
\hline Shutter type & Global shutter \\
\hline Modes of operation & $\begin{array}{l}\text { Double differential sampling (DDS) } \\
\text { Differential imaging (DI) }\end{array}$ \\
\hline Maximum data rate/master clock & $64 \mathrm{MPS} / 32 \mathrm{MHz}$ \\
\hline Supply voltage & $1.8 \mathrm{~V}$ \\
\hline Fill Factor & $16 \%$ \\
\hline Pixel type & $33 \mathrm{~T}(\mathrm{Custom}$ designed) \\
\hline Target frame rate & $30 \mathrm{fps}$ \\
\hline
\end{tabular}

Table 2.1: Sensor specifications.

The image sensor is operated in global shutter mode. In the global shutter operation, the entire imaging array is globally reset before integration and all rows start and stop integrating at the same time. A rolling shutter on the other hand has a start and stop time shifted for every row by one time unit with respect to the previous row. Global shuttering avoids image smear or distortion of fastmoving objects during readout as all the pixels are reset and integrated over the same interval. While rolling shutter is good for static or slow moving objects 
however they produces image smear and are incompatible with CDS (Correlated double sampling) processing.

\subsubsection{Pixel architecture}

A pixel can either be a passive pixel (PPS) or an active pixel. A passive pixel is composed of a photodiode and an addressing transistor. The PPS is very simple and compact however it suffers from noise problems due to the mismatch of the small photodiode and the large column capacitance. In contrast to a passive pixel, an active pixel (APS) incorporates a buffer (or amplifier) in the pixel which solves the noise problems due to the large column capacitances. The pixel used in the design is a smart pixel which is a form of an active pixel sensor. Smart pixels are able to perform parallel processing of large pixilated images, thus reducing the amount of signal sent off-chip. Although this type of pixel significantly improves the performance of the pixel, it severely limits the fill factor [2.18].

The desired functionalities of the compound eye of the insects namely, motion detection, high sensitivity to light intensity and polarization as described in section 1.2 are to be replicated in the pixel. The polarization is detected using the metals layers of standard CMOS and is described in detail in chapter 3. As discussed in the section 1.1, insects detect motion based on the change in state of its individual receptors creating a "flickering effect". To reproduce this effect, inpixel memories are needed to detect change in the state of the pixel by comparing current image with previous image. Further as the insects sees things as only "on" and "off", which digitally means ' 1 ' or ' 0 ', the stored images of the current and the previous image need to be binarized. Further to enhance the sensitivity to low light intensities, the dynamic range of the image sensor is extended using partial charge transfer mechanism. In partial charge transfer, the accumulated photoelectrons resulting from the incident illumination is sampled multiple times. The design choice to have in-pixel memories for detecting motion provides for storing the multiple samples obtained using partial charge transfer mechanism to enhance the sensitivity. In the following sub-sections the design of the pixel and the pixel operation is explained in detail.

Figure 2.15 shows the transistor level circuit diagram of the pixel. In the subsequent sections, a simplified version of the pixel architecture will be used to explain the pixel operation. 


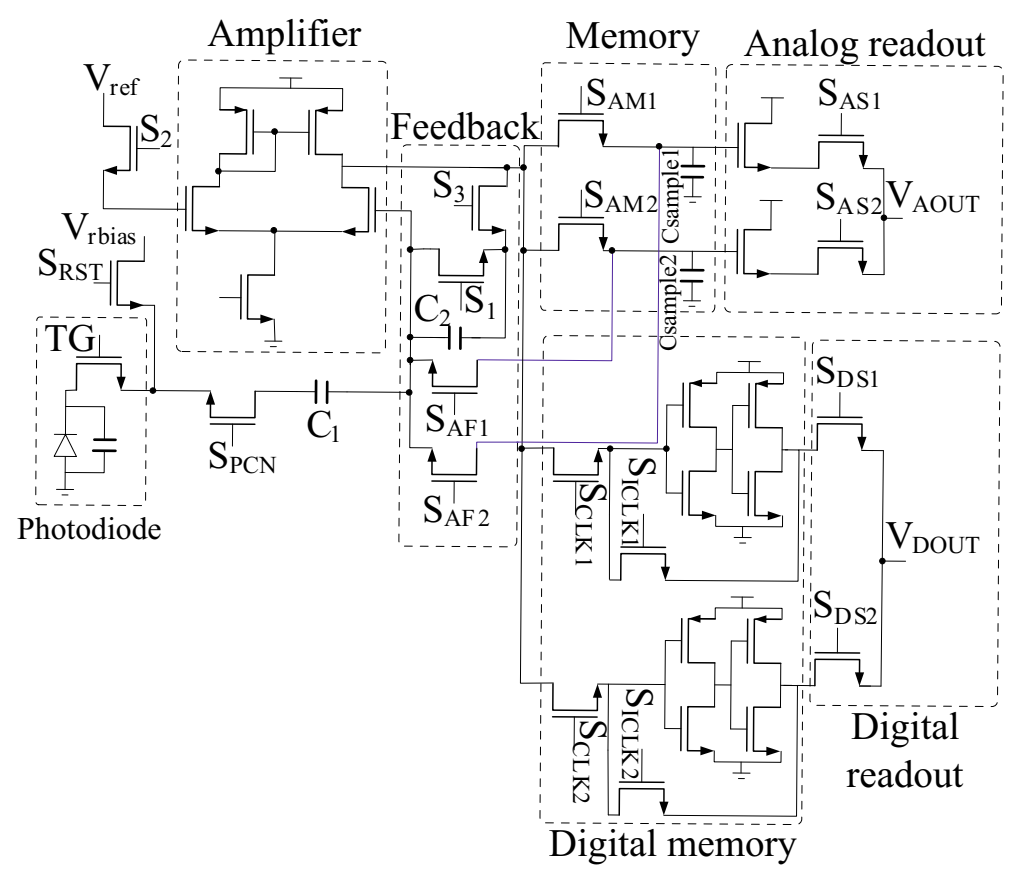

Figure 2.15: Pixel schematic.

\subsubsection{Pixel operation}

The operation of the pixel can be divided into four phases: image capture, image storage, digitization and readout. The image capture phase includes the reset of the photodiode and integration of the available photons in the photodiode. The image storage phase transfers the signal integrated at the photodiode from the sense node to the available analog memory. The digitization phase digitizes the stored analog signal. During the readout phase the data is transferred to the analog and digital signal processors via the respective column buses.

\subsubsection{Image capture phase}

The light sensing part used for the pixel is a $p^{+} / n / p$ pinned photodiode. The cross section of the pinned photodiode is shown in the figure 2.16, together with the transfer gate $(T X)$ and the reset switch $\left(S_{R S T}\right)$.

The image capture phase begins with a reset of the pixel by closing the $\left(S_{R S T}\right)$ switch. The voltage at the floating diffusion node $(F D)$ is set to the reset voltage 


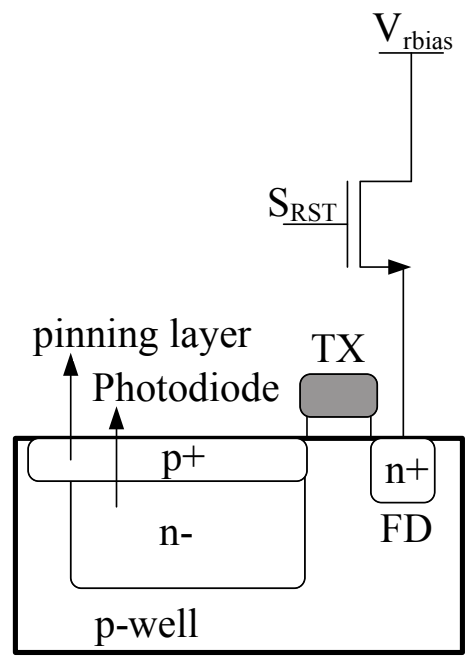

Figure 2.16: Pinned photodiode with reset.

$V_{\text {rbias }}$. To ensure that the reset transistor switches properly, the voltage $V_{R S T}$ at the gate of the reset transistor needs to be a threshold voltage $\left(V_{T H}\right)$ above $V_{\text {rbias }}$. This is also known as "hard reset". Another form of reset is the "soft reset" where the gate and the drain potential of the reset transistor are at the same level. Soft reset has a noise advantage over hard reset because the electrons in soft reset can only move uni-directionally from the photodiode to the reset transistor compared to the bi-directional motion of the electrons in hard reset. However a soft reset produces a higher image lag and also decreases the output voltage swing possible at the FD node thus hard reset is used in this design.

For the hard reset, $V_{\text {rbias }}$ can be expressed as

$$
V_{\text {rbias }}=-V_{R S T}-\left(V_{T H}+V_{\text {ovd }}\right)
$$

where $V_{T H}$ is the threshold voltage of the reset transistor (including body effect) and $V_{o v d}$ is its overdrive voltage. A NMOS transistor is used as the reset switch instead of a PMOS transistor in this design as a PMOS transistor needs an additional $n$-well, which would not only increase the pixel area but would also contribute to the noise by the additional electrons generated by the captured photons in the $n$-well. The reset transistor is a thick oxide transistor capable of handling $3.3 \mathrm{~V}$ provided by the $0.18 \mu \mathrm{m}$ CIS technology. The voltage $V_{R S T}$ is set to $3.3 \mathrm{~V}$ and $V_{\text {rbias }}$ at $1.8 \mathrm{~V}$ as $V_{T H}+V_{\text {ovd }}$ would not exceed $1.5 \mathrm{~V}$ for the chosen technology. The reset transistor is designed with minimum dimensions ensuring 
that there are few charges in the channel when it is conducting, and reducing the charge injection when it is turned off. When $S_{R S T}$ is closed (connected) the transfer gate $T X$ is also closed, to effectively reset the photodiode to its pinning voltage. For the designed photodiode the pinning voltage is at $1 \mathrm{~V}$. The transfer gate has a channel width of $10 \mu \mathrm{m}$ and a channel length of $1 \mu \mathrm{m}$. The wider transfer gate helps the charge transfer from the photodiode to the floating diffusion node. The floating diffusion capacitance must be large enough to hold all the charges transferred from the photodiode. However, it cannot be made too large as it decreases the conversion gain.

When the transfer gate opens, the photodiode starts accumulating the photogenerated charge. The time spent accumulating charge is referred to as integration time or exposure period. At the end of the integration time, the accumulated charge is transferred to the $F D$ by closing the transfer gate $T X$. Figure 2.17 shows the potential diagram of the pinned photodiode for complete charge transfer. When the photodiode is fully depleted, after the charge transfer it returns to its pinning voltage as shown in figure 2.17(a). After the photodiode is reset to its pinning voltage, charges start to accumulate on the photodiode as shown in figure 2.17(b). The amount of charge collected is directly proportional to the illumination level till the photodiode reaches saturation when the full well is reached.
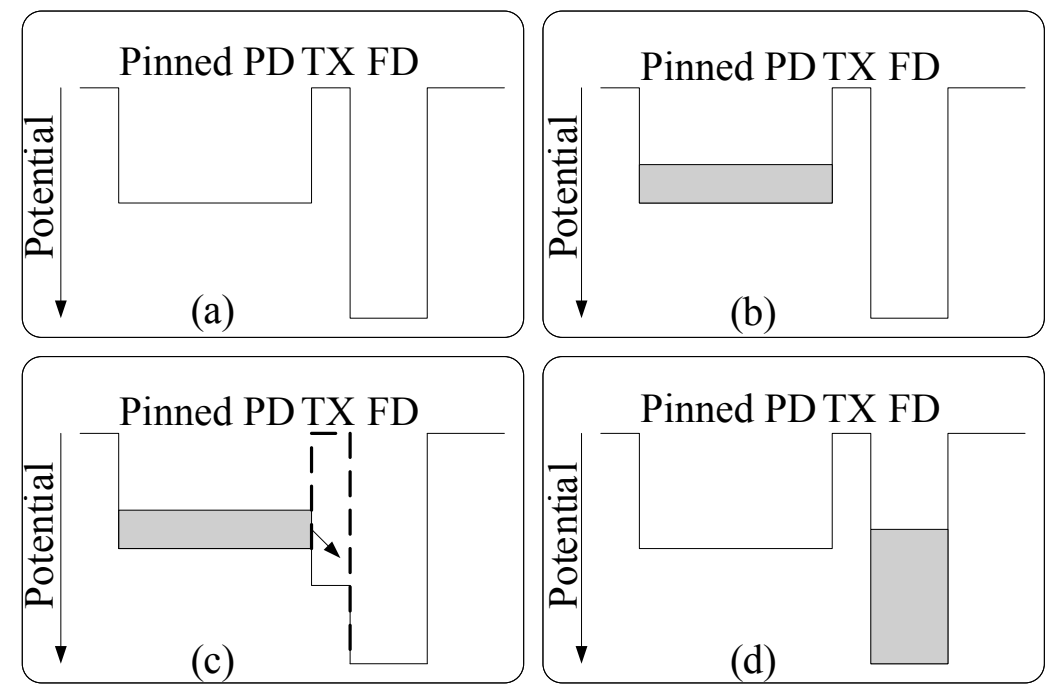

Figure 2.17: Pinned photodiode operation a) before charge integration, $b$ ) during and after charge integration, c) during charge transfer, d) after charge transfer. 
At the end of the integration period, the transfer gate $T X$ is closed, which allows the transfer of accumulated charges from the photodiode into the floating diffusion node as shown in figure 2.17(c) and (d).

Besides a complete charge transfer, the photodiode can also transfer accumulated charges partially after each exposure period. This is known as partial charge transfer mode of operation, where the total exposure period is composed of multiple shorter exposure periods. After each small exposure the accumulated charges in the photodiode are transferred to the $F D$ node. The operation of the pixel in the partial charge transfer mode when sampled twice is shown in figure 2.18 .

Figure 2.18(a) shows the depleted state of the photodiode. The charge is accumulated (figure 2.18(b)), and after a certain accumulation time $\left(T_{1}\right)$ the transfer gate is closed (figure 2.18(c)) transferring the accumulated charge into the floating diffusion (figure 2.18(d)). After the first transfer, the photodiode continues to accumulate charge until the end of the exposure time $\left(T_{F T}\right)$. The accumulated charge is transferred to the floating diffusion by closing the transfer gate as shown in the figure $2.18(\mathrm{e})-(\mathrm{h})$. Thus the charges are transferred to the $F D$ at two time instances: $\left(T_{1}\right)$ and $\left(T_{F T}\right)$.

\subsubsection{Image storage phase}

The simplified circuit for the image storage phase along with the corresponding timing diagram is shown in the figure 2.19. In the image storage phase, the voltage change from the reset level at the $F D$ node due to the transferred photocharge is sampled onto one of the two available sampling capacitors $C_{\text {sample }}$ when the switch $S_{A M}$ is closed in figure 2.19 .

The amplifier $A M P$ used is a simple differential amplifier. It serves two functions: amplification and comparison. When $S_{3}$ is closed, the amplifier works in closed loop configuration. When the analog signal voltages at the sense node are sampled onto the analog memories, the amplifier amplifies the signal at the sense node by the closed loop gain provided by the capacitors $C_{1}$ and $C_{2}$. The pixel is designed with a closed loop gain $\left(A_{P I X E L}\right)$ of 7 . The switch $S_{2}$ connects the globally distributed reference voltage $V_{\text {ref }}$ to the non-inverting terminal of $A M P$ and the switch $S_{P C N}$ is used to connect or disconnect the pinned photodiode to the the $A M P$.

Autozeroing is used to reduce the effects of the input-referred dc offset voltage $\left(V_{o f f}\right)$. When $S_{1}$ and $S_{3}$ are closed, the amplifier is connected as a voltage follower and the offset voltage $V_{\text {off }}$ is stored on the capacitor $C_{1}$. The charge 

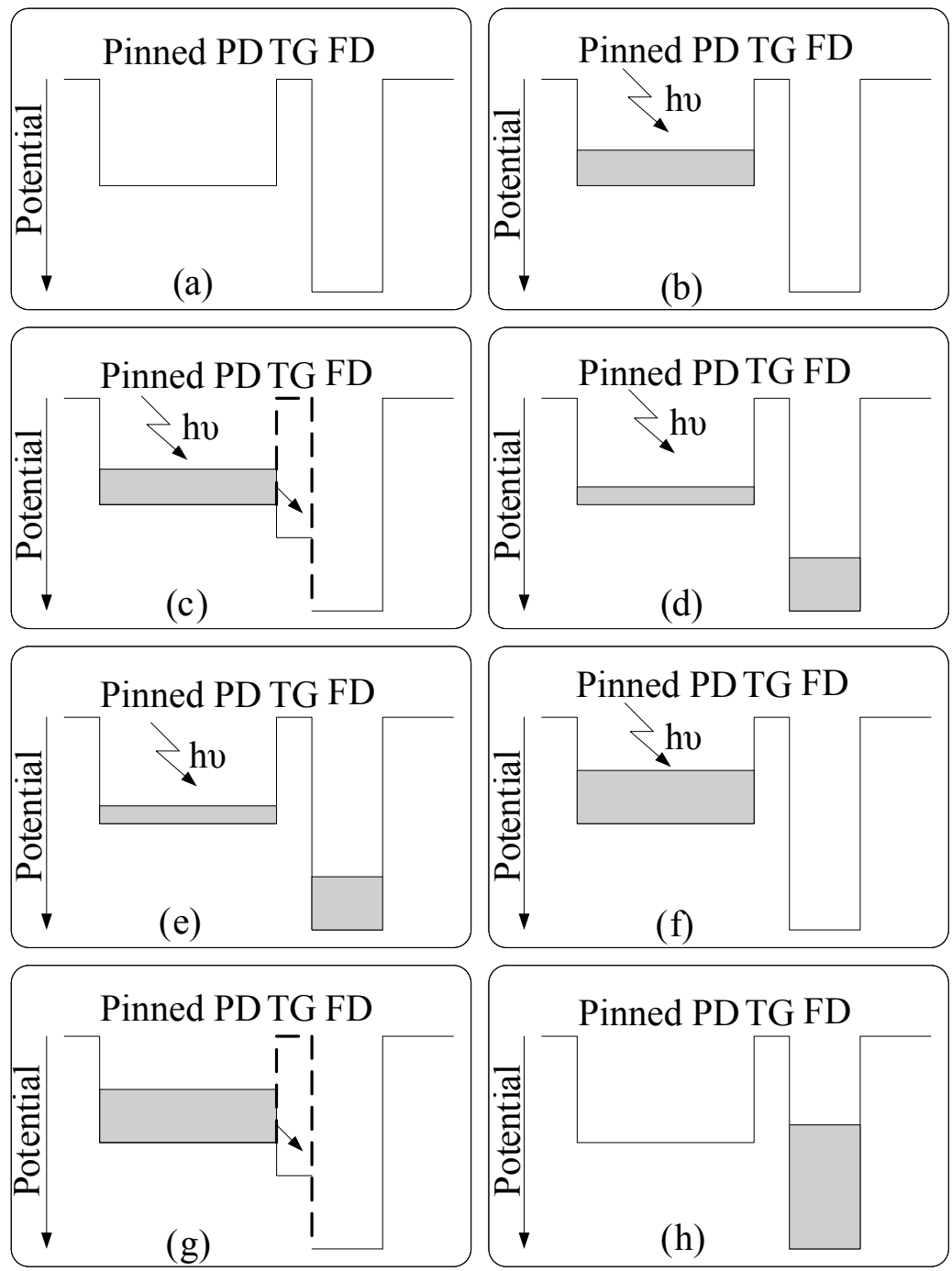

Figure 2.18: Pinned photodiode operation in partial charge transfer mode.

equations when $S_{1}$ and $S_{3}$ are closed can be written as

$$
Q_{1}=C_{1}\left(V_{\text {ref }}+V_{o f f}-V_{\text {rbias }}\right)+C_{2} V_{o f f}
$$

where $Q_{1}$ is the offset charge, $V_{\text {ref }}$ is the reference voltage applied to the noninverting terminal of $A M P, V_{o f f}$ is the offset voltage, $V_{\text {rbias }}$ is the reset voltage and $C_{1}$ and $C_{2}$ are the capacitors. When the signal is sampled, switch $S_{1}$ is 

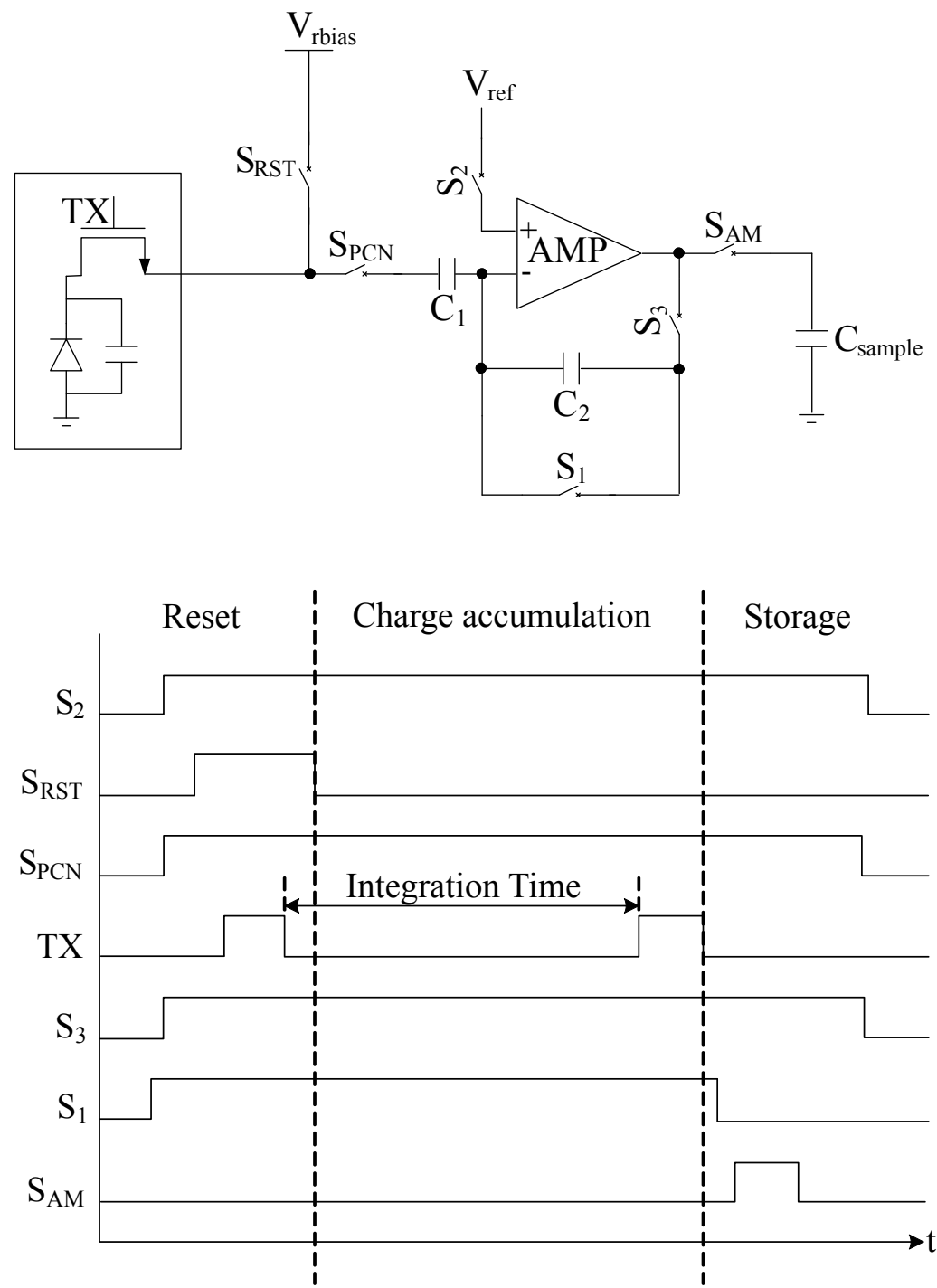

Figure 2.19: Pixel in image storage phase and associated timing diagram.

opened and the charge equation can be written as

$$
Q_{2}=C_{1}\left(V_{r e f}+V_{o f f}-V_{\text {sig }}\right)+C_{2}\left(V_{\text {ref }}+V_{\text {off }}-V_{\text {out }}\right)
$$

Because the change in charge in the capacitors is reflected in the output voltage 
$V_{\text {out }}$, the output voltage can be derived from equation (2.5) and (2.6) and is free of offset voltage of the amplifier.

$$
V_{\text {out }}=\frac{C_{1}}{C_{2}}\left(V_{\text {rbias }} V_{\text {sig }}\right)+V_{\text {ref }}
$$

The designed image sensor can be operated in two modes: double differential mode $(D D S)$ and differential imaging mode $(D I)$. In case of $D D S$ each pixel samples the voltage at the $F D$ node twice: once after reset and again after the exposure period and it stores the sampled values in the two available analog memories. In the differential mode two samples are stored in the analog memories after different exposure periods. The $D D S$ mode of operation is further elaborated in section 2.5.5.1 while the DI mode of operation is explained in chapter 6 .

\subsubsection{Image binarization phase}

The simplified schematic of the pixel in the image binarization phase and its associated timing diagram is shown in the figure 2.20.

After an image is captured, the photodiode is disconnected from the processing elements by opening the switch $S_{P C N}$. The analog signal sampled on $C_{\text {sample }}$ is compared with a fixed reference voltage $V_{\text {ref }}$ when the switch $S_{A F}$ is closed, by using the amplifier as a comparator. The globally distributed reference voltage $V_{r e f}$, is connected to each pixel's comparator non-inverting input while the feedback signal stored in the analog memory is connected to the inverting input of the comparator. The resulting binary output voltage is stored in the 6T-SRAM cell when the switch $S_{C L K}$ is closed. Two such SRAM cells are available in each pixel to store the binary data of the two sampling capacitors. Writing into the memory is locally controlled by the comparator. The switch $S_{A M}$ is always open in the binarization phase.

\subsubsection{Image readout phase}

The final phase of the pixel operation is the readout phase when each pixel is read out sequentially. The source follower $S F$ loads the column bus $V_{A O U T}$ via the analog row selection switch $S_{A S}$ with the voltage sampled on the sampling capacitor during readout of the pixel as shown in figure 2.18. The switch $S_{D S}$ loads the column bus $V_{D O U T}$ with the binary value stored in the SRAM cell. The 

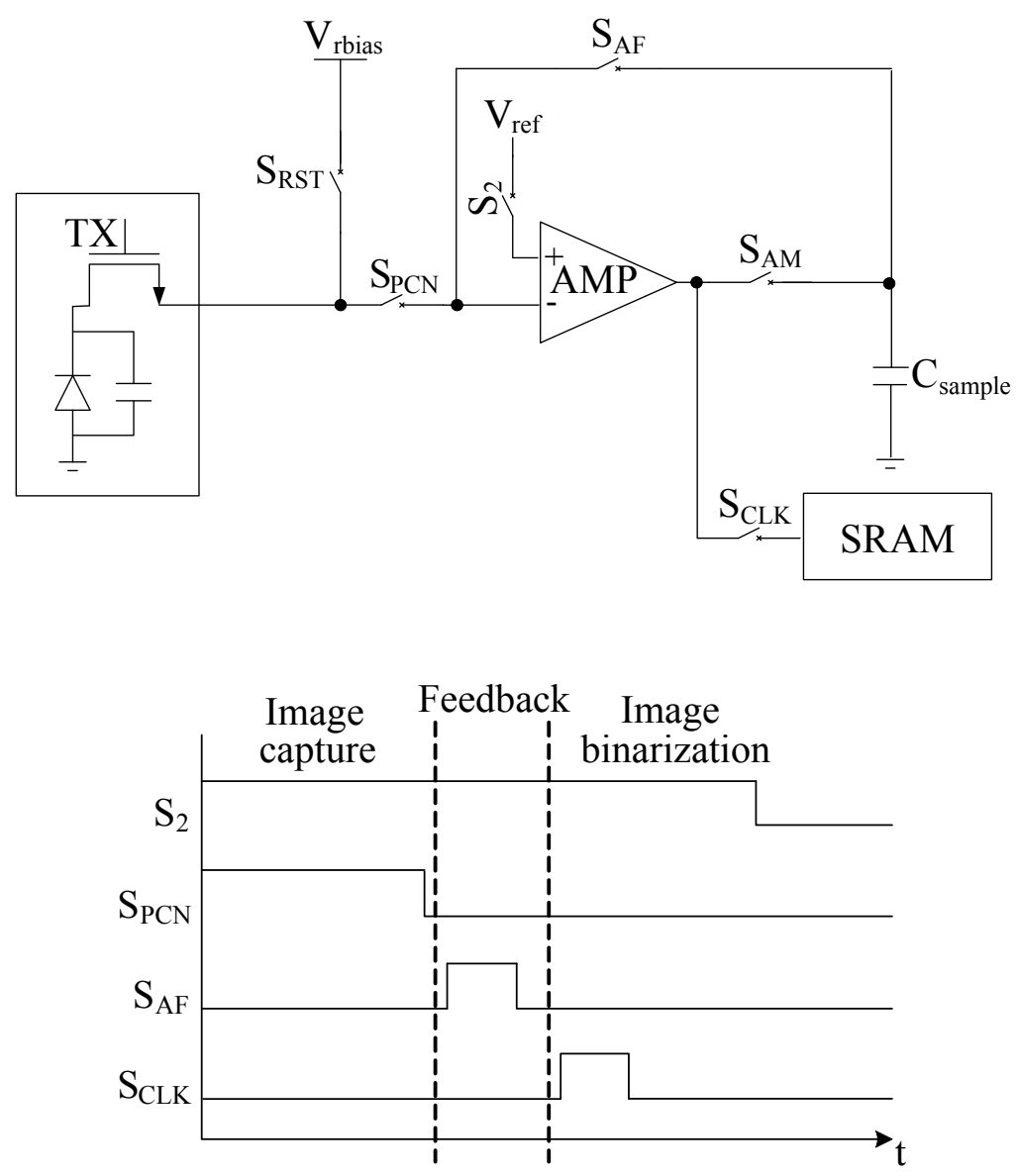

Figure 2.20: Pixel in image storage phase and associated timing diagram.

control signals for switches $S_{A S}$ and $S_{D S}$ are generated by the row addressing logic explained in section 2.5.4.

\subsubsection{Pixel layout}

Figure 2.22 shows the layout of a single pixel block. The large square at the center is the pinned photodiode with a dimension of $10 \mu \mathrm{m}$ by $10 \mu \mathrm{m}$. The analog memories $C_{\text {sample }}$ are implemented with Metal-Metal (MiM) capacitors and are placed below the photodiode in figure 2.22. The amplifier/comparator is placed on the left hand side while the digital memory is placed on the right hand side. 


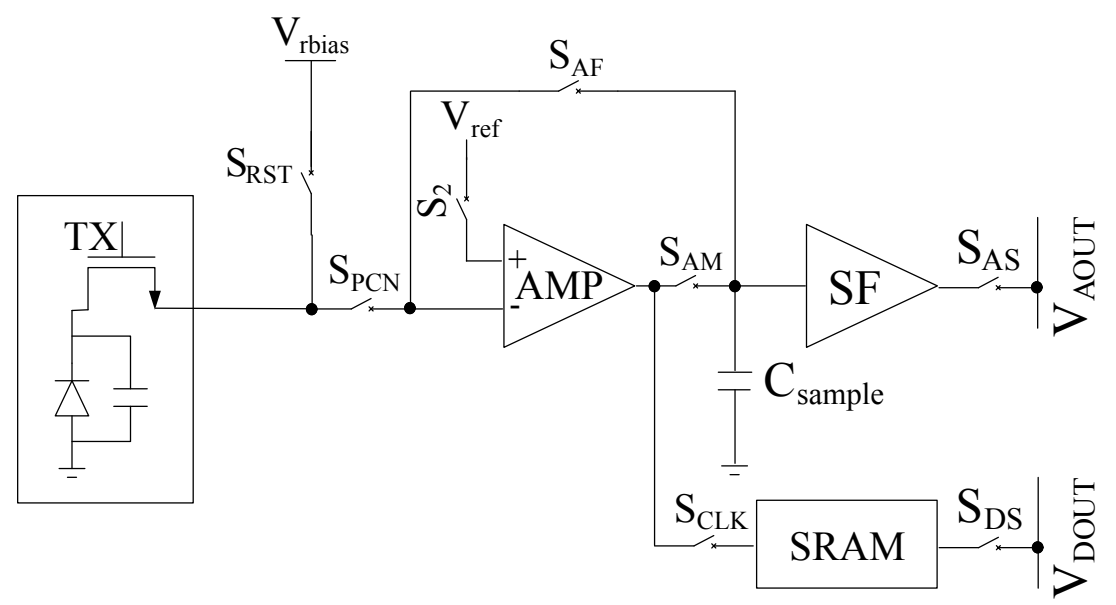

Figure 2.21: Pixel (Image) readout phase.

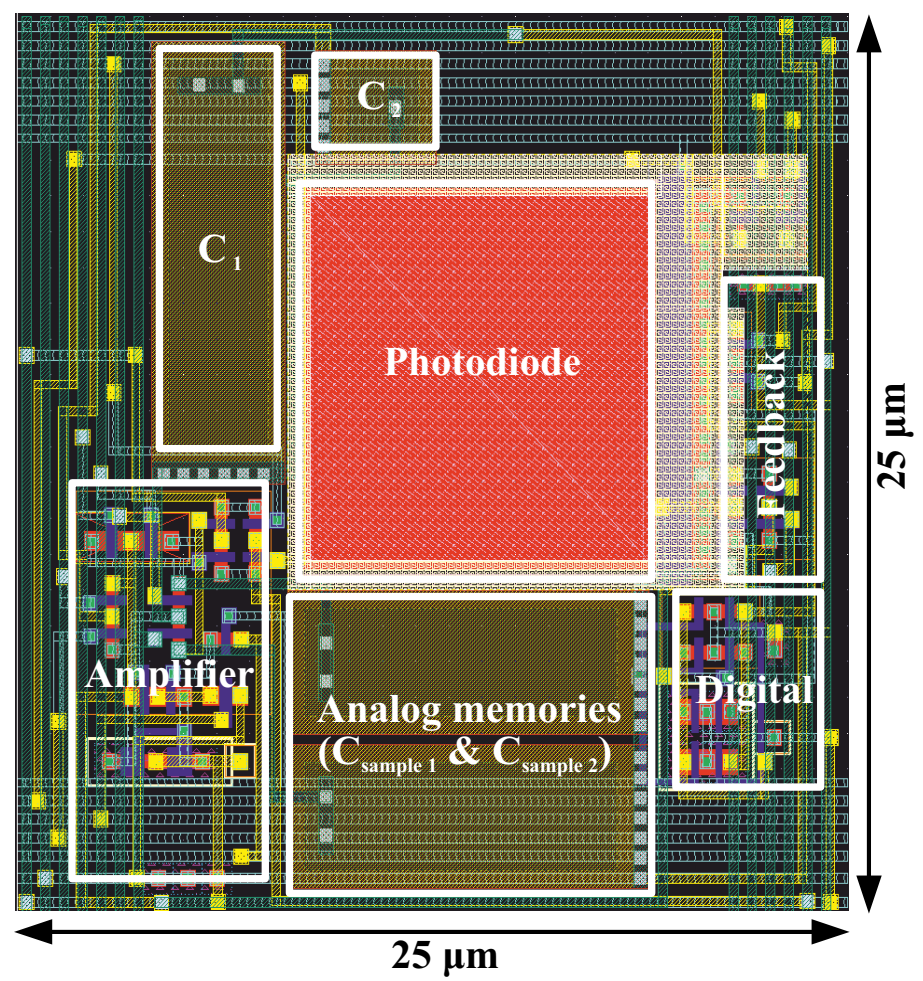

Figure 2.22: Pixel schematic and layout. 


\subsubsection{Row/Column addressing logic}

Several architectures for pixel addressing are available in CMOS image sensors. The row select logic used in the designed image sensor is column parallel readout architecture. Column parallel readout architecture for a $128 \times 128$ pixel array is shown in figure 2.23. The row address block generates the row select pulse to select a row and the column address pulse generates the column select pulse to select the column in the selected row to be read.

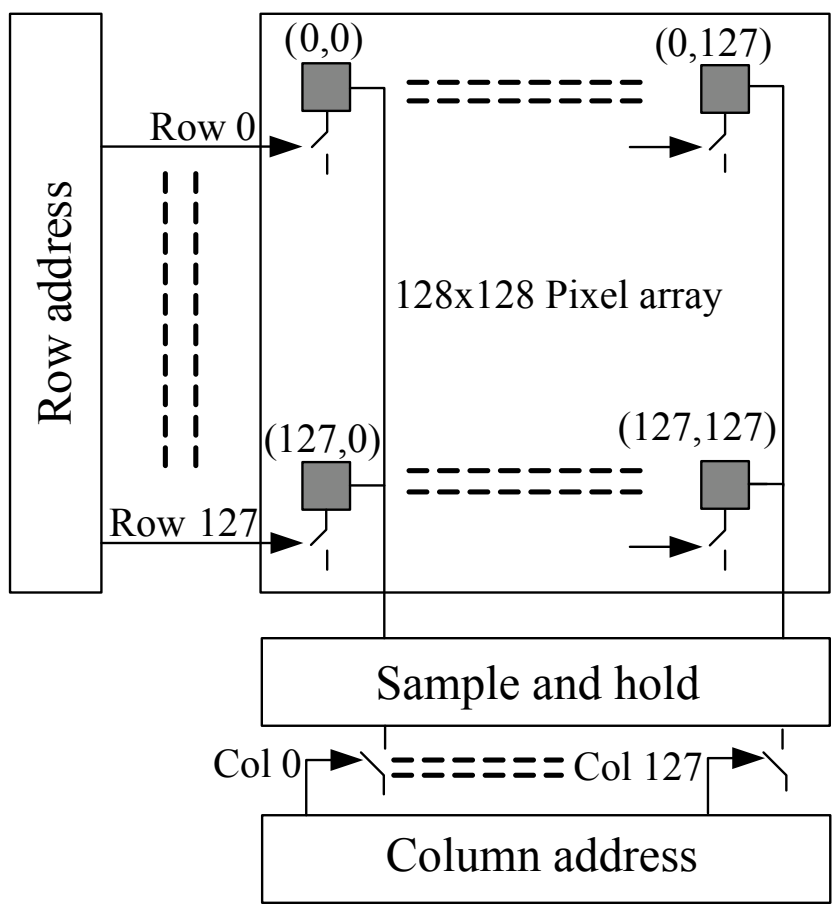

Figure 2.23: Column parallel readout architecture.

The column parallel readout is progressive in nature. Progressive scan readout of an area format CMOS image sensor involves selecting a row and simultaneously reading and processing all pixels in the selected row. Column readout is sequential in nature for the designed imager, where each pixel is readout serially. The progressive scan readout sequence is shown in the figure 2.24.

In image sensors, two readout control structures are commonly used to generate the control signals for selecting the columns and the rows: shift registers and 


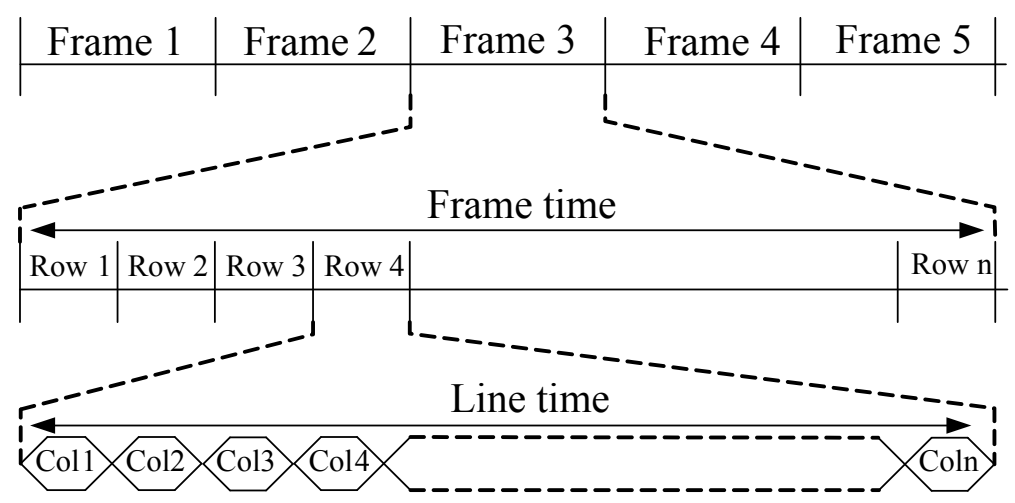

Figure 2.24: Progressive scan readout.

decoders. The advantage of using shift registers over decoders is that shift registers are easy to implement and use fewer transistors. However random access to pixels is not possible with shift registers as they produce only sequential output from the first element to the last one. Decoders are preferred for random access readout. However in this design shift registers are preferred for its simplicity in implementation. In this design, the row selection logic circuit is composed of a non-overlapping clock generator block and a shift register block, which is a cascade of shift registers. Figure 2.25 shows the implemented shift register.

The shift register is a serial-in serial-out type. The data is delayed by one clock cycle at each stage. PHII and PHI2 are non-overlapping clocks. When the clock PHII is high the input signal to the shift register $V_{I N}$ is saved in the INVI and $I N V 2$ loop. This saved signal is shifted out as an output $V_{O U T}$ when $P H I 2$ is high via the INV3 and INV4 loop.

The non-overlapping clock signals $P H I I$ and $P H I 2$ are generated on-chip using the non-overlapping clock generation circuit shown in the figure 2.26. The non-overlapping clock generation circuit is implemented with $N A N D$ gates and inverters. The non overlapping clocks $P H I 1$ and $P H I 2$ are fed to the shift register block to shift the row read control signal $R O W \_R D \_S I G N A L$ to generate sequential outputs to read one row at a time. Both the analog signal processor and the digital signal processor in figure 2.14 have individual column select pulses generation circuit to select one column at a time. These circuits are similar to those used in the row select logic. 

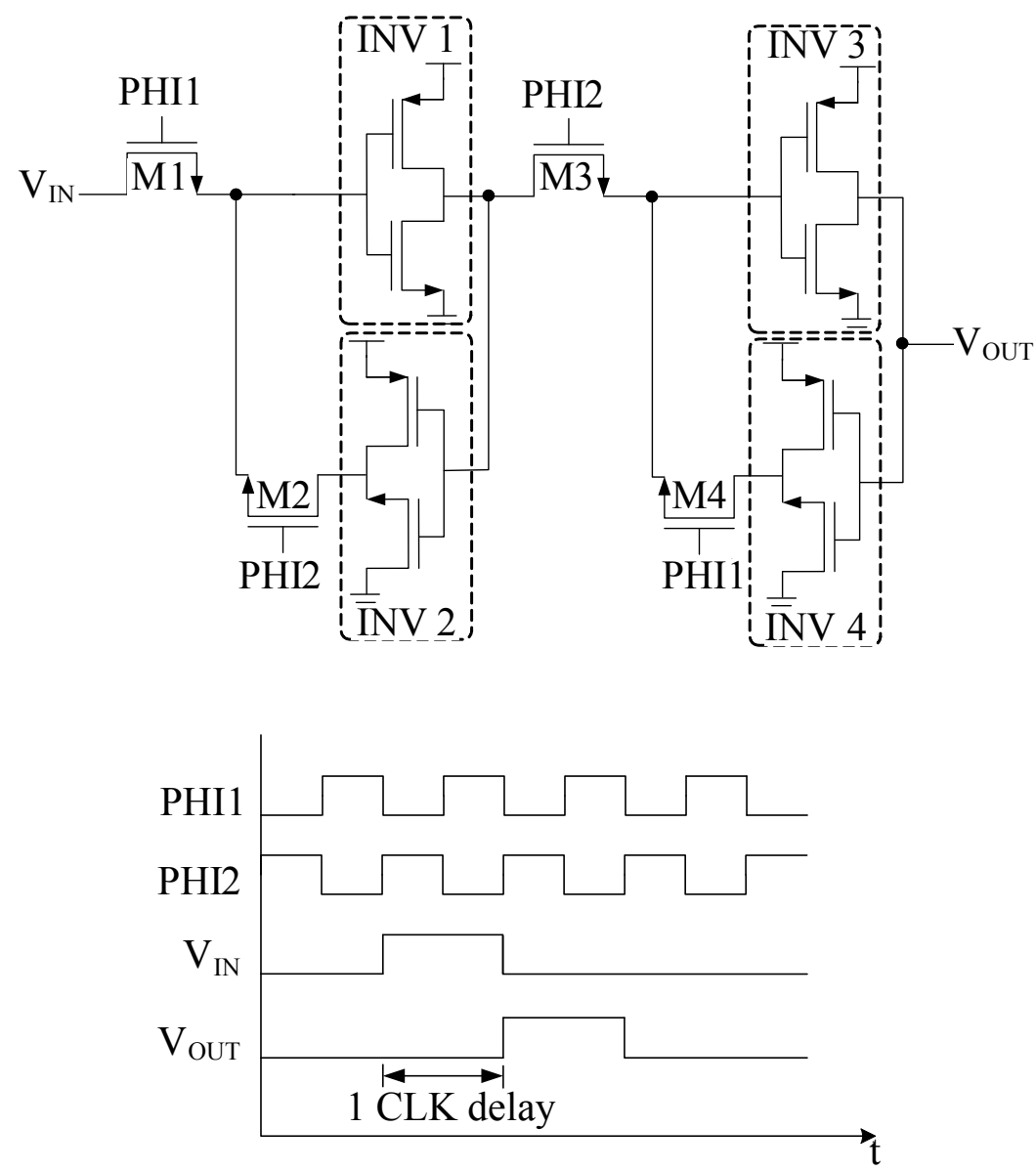

Figure 2.25: Shift register and associated clock timing.

\subsubsection{Analog signal chain}

The simplified analog signal chain is shown in the figure 2.27. In the analog signal chain the analog signals stored in the analog memories $C_{\text {sample } 1}$ and $C_{\text {sample } 2}$ in the pixel are transferred to the sample and hold capacitors $C_{1}$ and $C_{2}$ in the column differential sampling circuit when the pixel is read. The stored signals in the sample and hold capacitors are buffered to the output amplifier $A 3$. The differential output generated by $A 3$ is then buffered to the chip output pad using the voltage follower $A 4$. 


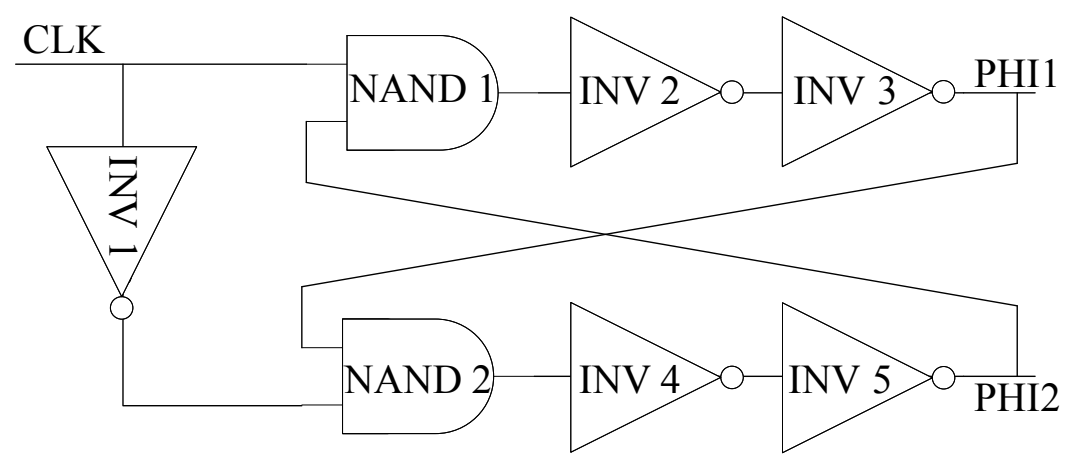

Figure 2.26: Non-overlapping clock generation circuit.

\subsubsection{Delta differential sampling (DDS)}

Delta differential sampling is based on using two successive track-and-hold $(T / H)$ operations to reduce the low frequency noise and fixed pattern noise inherent in standard CMOS image sensor outputs. DDS reduces noise by sampling each pixel twice, once immediately after reset and again after exposure. The array is readout row wise, where the reset and the signal levels in the in-pixel memories are transferred to the column circuits one row at a time. However since all the pixels are reset at the same time the imaging array as a whole has a global shutter. By storing the pixel value after reset in a track-and-hold circuit, the pixel level fixed pattern noise can be subtracted from the actual pixel signal at exposure for a very accurate measurement.

The schematic of the sample and hold circuitry used in the designed image sensor is shown in figure 2.28. The signal on the pixel analog column bus $V_{A O U T}$ is sampled to $C_{1}$ when both the $S_{S H R}$ switch and the row select switch $S_{A S 1}$ are closed and to $C_{2}$ when the $S_{S H S}$ switch and the row select switch $S_{A S 2}$ are closed. The crowbar switch $S_{C A L}$ is opened when the pixel output line is sampled on the sample and hold capacitors.

The bottom plates of the sample and hold capacitors are connected to the externally generated reference voltages $V_{R E F_{-} R}$ and $V_{R E F_{-} S}$ to reduce the substrate noise [2.19]. As the bottom plates of the sample and hold capacitor sees a fixed reference voltage, the substrate noise through the the parasitic capacitor between the bottom plate and the substrate is of little importance. The voltage values used for $V_{R E F_{-} R}$ and $V_{R E F_{-} S}$ is $500 \mathrm{mV}$, to provide enough bias voltage to the voltage followers $A 1$ and $A 2$. The crowbar switch $S_{C A L}$ shorts the two storage 


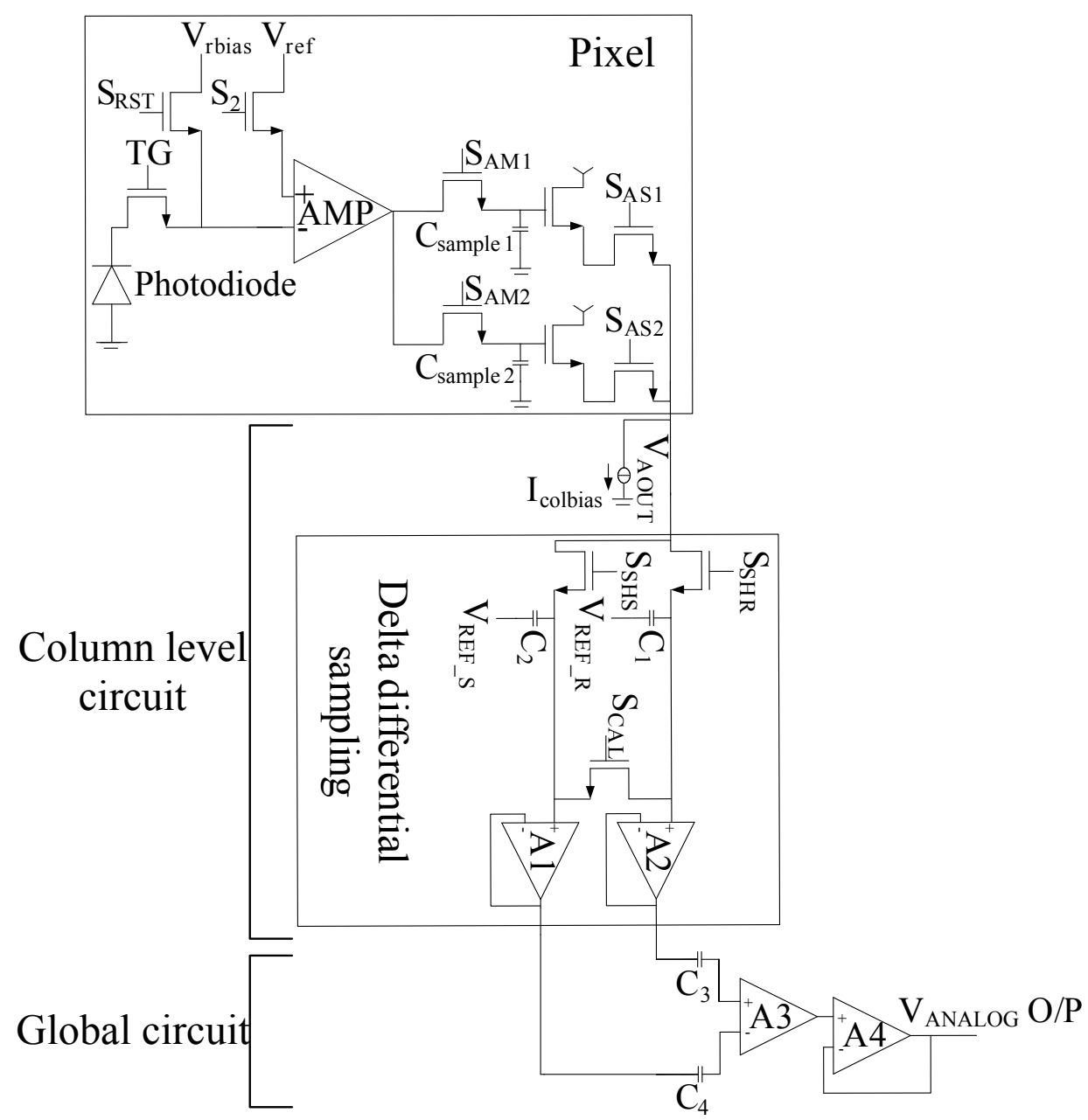

Figure 2.27: Analog signal chain.

capacitors to compensate for the offsets of the voltage followers $A 1$ and $A 2$.The timing diagram for the differential sampling operation and the $S_{C A L}$ switch clock timing to read one row is shown in the figure 2.29.

The minimum value of the sample and hold capacitors are determined by $k T C$ noise limitations. A 500fF hold and sample Metal-Metal capacitor is chosen, which gives a noise of $90 \mu \mathrm{V}$. The output of the column sample and hold capacitors are fed into the output amplifier through the voltage followers $A 1$ and $A 2$. The voltage followers $A 1$ and $A 2$ are similar to the $O T A$ used in the pixel to 


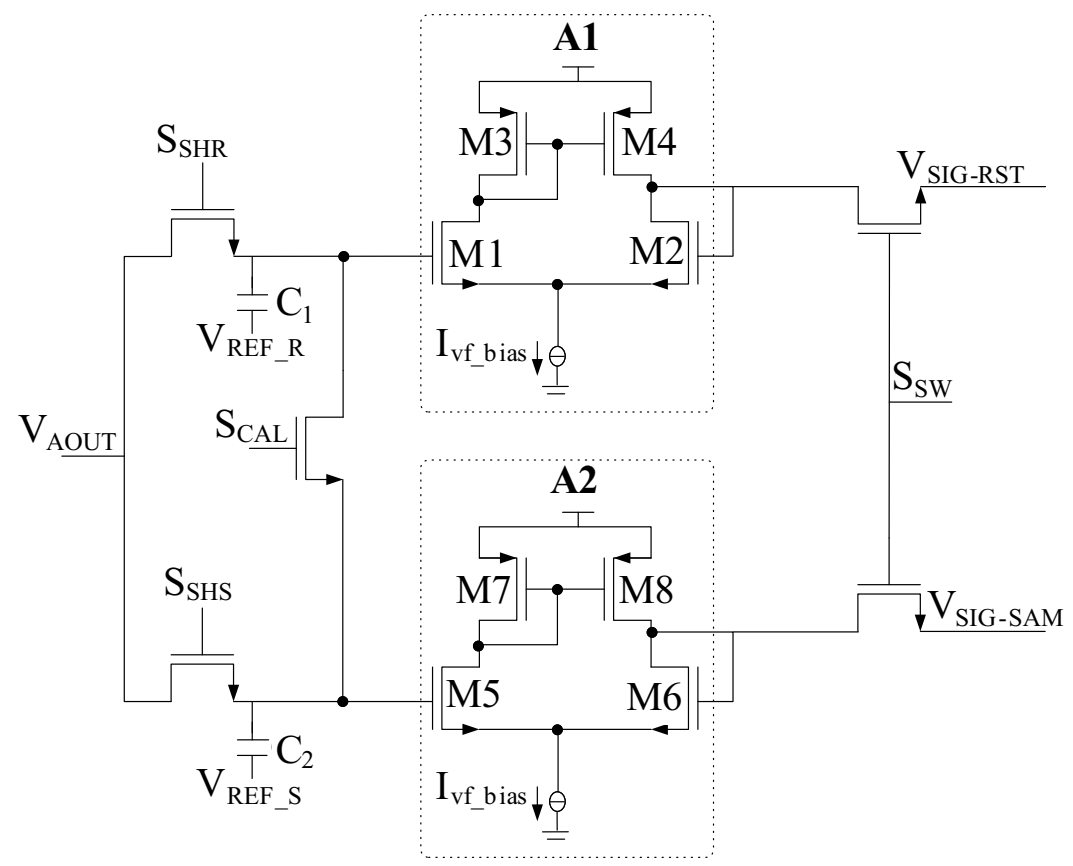

Figure 2.28: Analog signal chain.

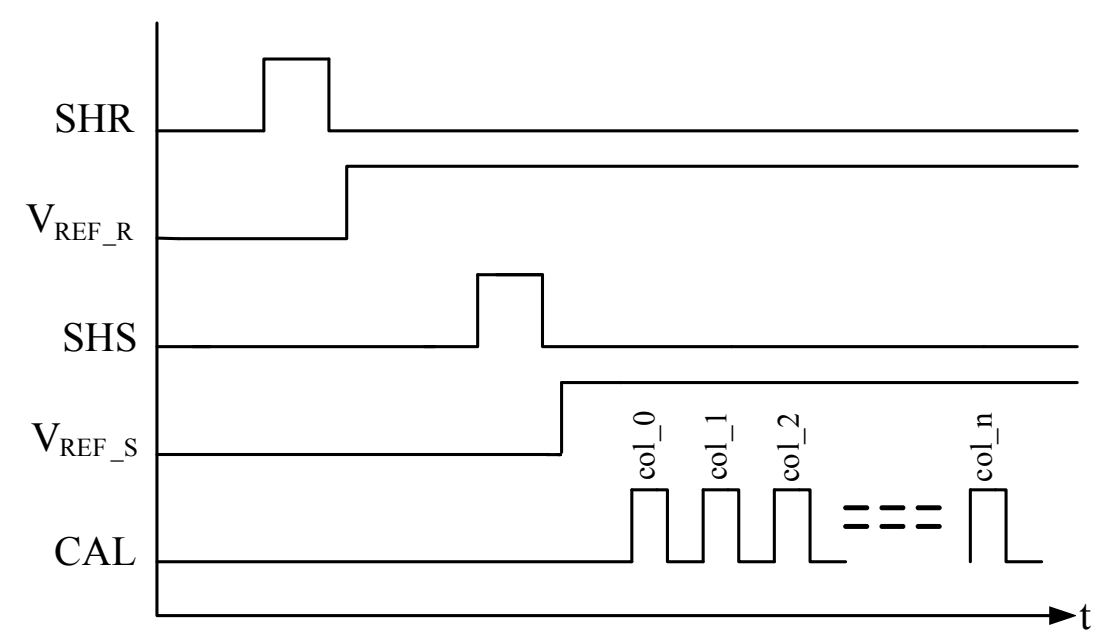

Figure 2.29: DDS timing diagram.

shorten the design time. 
The crowbar operation is initiated when $S_{C A L}$ is closed. After the sampling of the column output on the sample and hold capacitor, the $S_{C A L}$ switch is closed shorting the two capacitors. The output of the differential output amplifier changes depending on the voltage difference sampled on the sample and hold capacitors. The switch $S_{S W}$ is used to transfer the voltages from the delta differential circuit to the output amplifier circuit.

\subsubsection{Output amplifier}

The overall block diagram of the output differential amplifier $A 3$ is shown in figure 2.30.The signals $V_{S I G_{-} S A M}$ and $V_{S I G_{-} R S T}$ are the signals in the sample and hold capacitors of the delta differential sampling circuit. The signals are stored on the left plates of the capacitors $C_{3}$ and $C_{4}$ respectively as shown in figure 2.30. Before the differential signals $V_{S I G_{-} S A M}$ and $V_{S I G_{-} R S T}$ are amplified, the amplifier $A 3$ is connected in autozero mode to store the offset on the top plate of the capacitor $C_{6}$. Figure 2.30 also shows the clocking diagram of the switches $S_{M 1}, S_{M 2}$ and $S_{C A L}$ (from delta differential circuit block) and the corresponding variations in the signals in the sample and hold channels along with the obtained $V_{A N A L O G}$ output. The $S_{M 1}$ and $S_{M 2}$ transistors have minimum channel dimensions to reduce the charge injection when the switches are closed.

The amplifier A3 is a simple differential OTA with an $N$-type input stage. The values of $C_{3}$ and $C_{4}$ are 250fF. The feedback capacitor $C_{6}$ sets the voltage gain. $C_{6}$ is chosen to be $125 \mathrm{fF}$, to provide for a closed loop gain $\left(A_{O U T P U T}\right)$ of 2. A $125 \mathrm{fF}$ capacitor $C_{5}$ couples the DC reference voltage $V_{R E F 2}$. The reference voltages $V_{R E F 1}$ and $V_{R E F 2}$ are generated externally.

\begin{tabular}{|l|l|}
\hline$C_{3}, C_{4}$ & $250 \mathrm{fF}$ \\
\hline$C_{5}, C_{6}$ & $125 \mathrm{fF}$ \\
\hline Gain $\left(A_{\text {OUTPUT }}\right)$ & 2 \\
\hline
\end{tabular}

Table 2.2: Design values for figure 2.30.

\subsubsection{Digital signal chain}

The simplified digital signal chain is shown in figure 2.31. The binary data from the two SRAM cells is loaded onto the digital column bus DOUT. The tri-state buffer is used to invert the incoming digital signal and the $R S$ flip flop synchronizes the tri-state buffer output for the counter. A 7-bit counter counts 

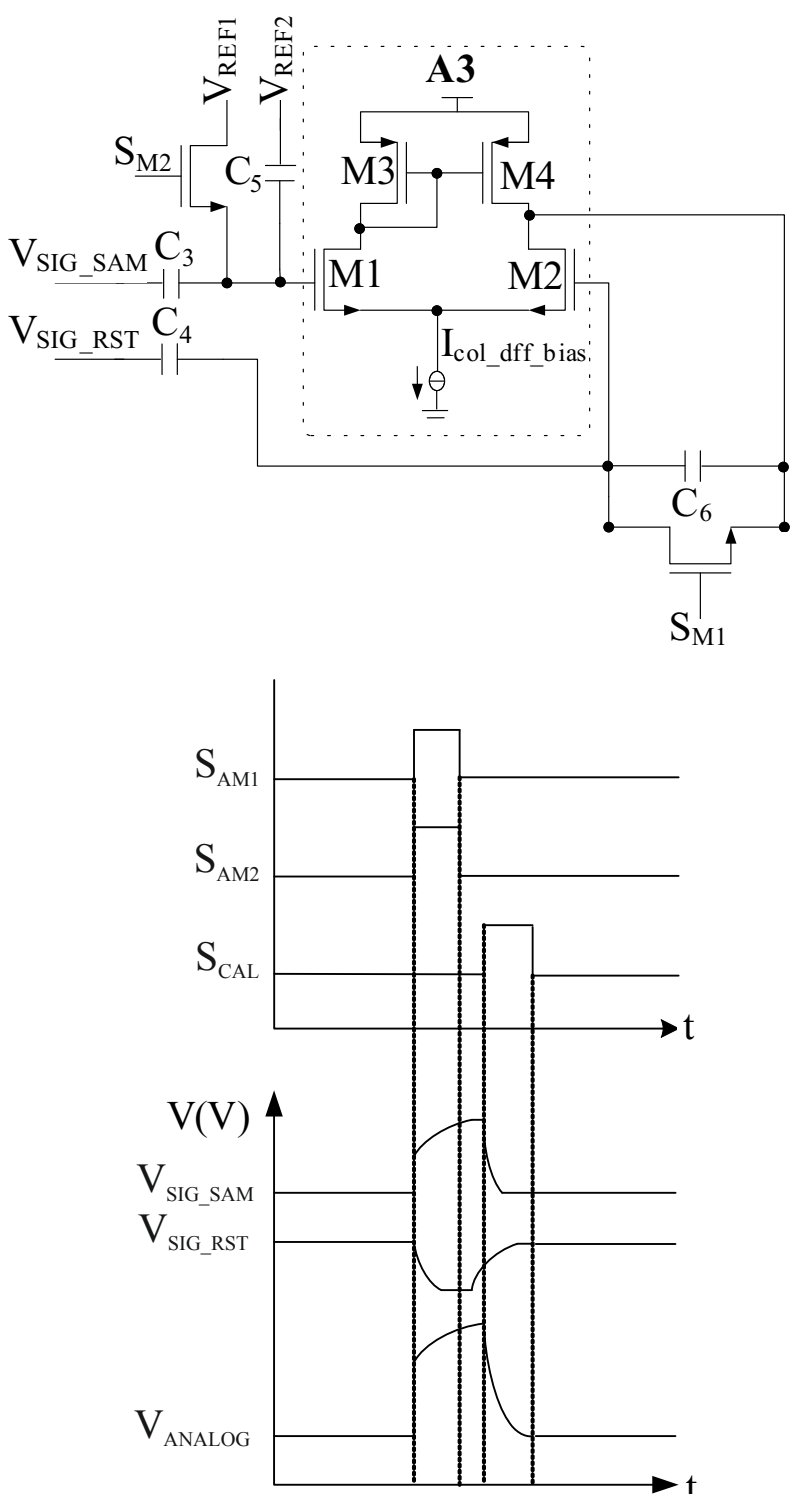

Figure 2.30: Output amplifier and associated timing and signal voltages.

the number of active high pixels in each row. The outputs of the counter and the digital output are buffered out to the chip output pins. 


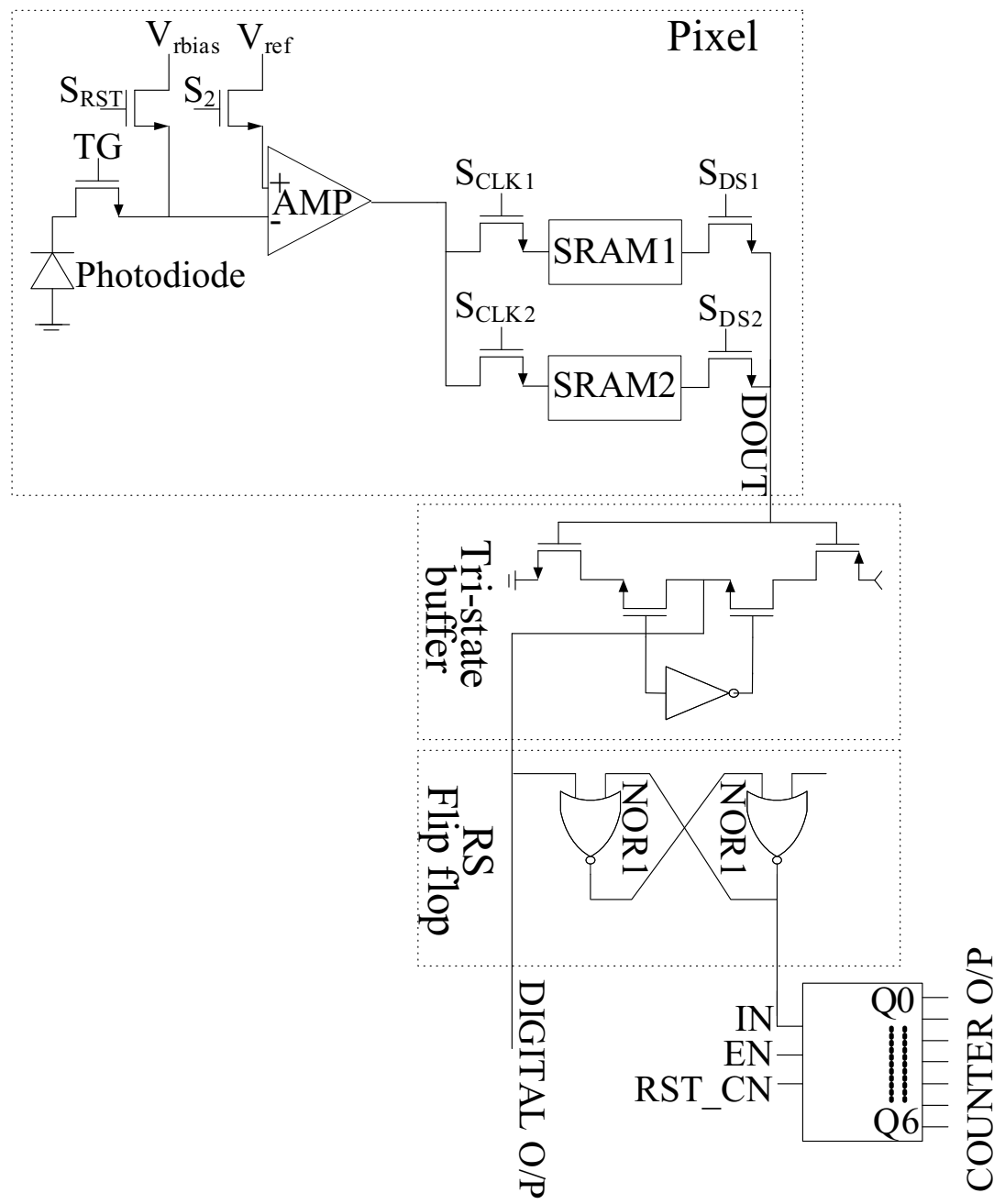

Figure 2.31: Digital signal chain.

\subsubsection{Digital counter}

The designed counter is a synchronous up counter with an reset input. The reset option allows resetting the counter to an all-zero state when desired. The 7bit digital counter is implemented using 7 modified $J$ - $K$ flip flops with their input shorted for toggle operation and additional $A N D$ gates as shown in the figure 2.32. The outputs of the counter $Q_{0}, Q_{1}, Q_{2}, Q_{3}, Q_{4}, Q_{5}$ and $Q_{6}$ are available for external interface to the FPGA for digital processing. 


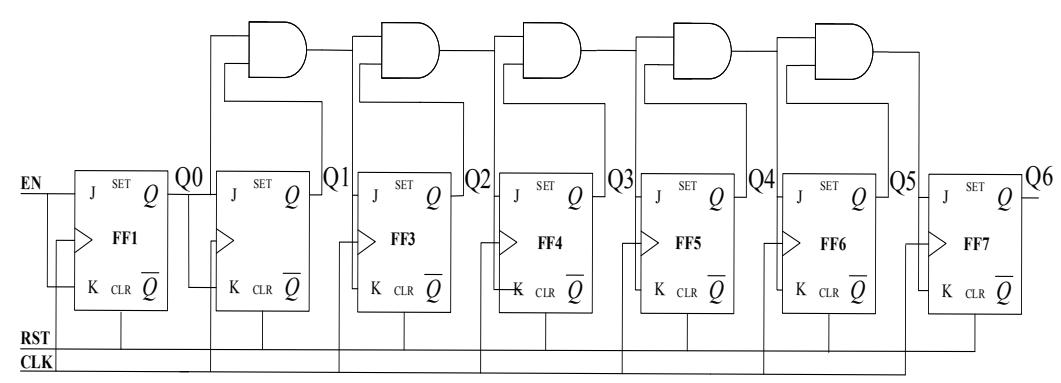

Figure 2.32: Digital counter.

The $J-K$ flip flop has been designed following a master/slave approach. Another alternative could be edge sensitive flip flops. However for the edge sensitive flip flops to work properly sharp clock edges are required and the threshold voltage of inverters and other gates be adjusted properly. Also the delay time through the two inverters at the input is critical. For these problems with clock and timing requirements, edge-sensitive flip-flops are not used in the design. The $J$ - $K$ flip flop can easily be toggled and thus is used in the counter in our design.

The output of the counter is all zero when the $R S T \_C N$ (RST to the $J-K$ flip flop) is held at logic high.

The pixels in the column are read out sequentially. When the digital output from each pixel in the column selected by the column select signal is high and the $R S \_C L K$ of the $R S$ flip flop is low then the output of the $R S$ flip flop is set high which increments the counter.

\subsection{Image sensor characterization}

A chip micro-photograph of the fabricated image sensor is shown in figure 2.33. The chip was manufactured in a $0.18 \mu \mathrm{m}$ 1-poly 3-metals CIS CMOS image sensor technology. The imaging array has $128 \times 128$ pixels with a pixel pitch of $25 \mu \mathrm{m}$. The chip size is $5 \mathrm{~mm} \times 4 \mathrm{~mm}$. The device is enclosed in a 68 pin JLCC (J Leaded Chip Carrier) package with a size of $25 \mathrm{~mm} \times 25 \mathrm{~mm}$. The chip also contains test structures which are used for testing pixels with different shapes of pinned photodiodes.

The characterization parameters for an image sensor are related to the parameters of the image quality and also the intended application. The parameters of interest for our application are the photoconversion characteristics, the sensitivity, the photon transfer curve to determine the conversion gain, and the dark current. 


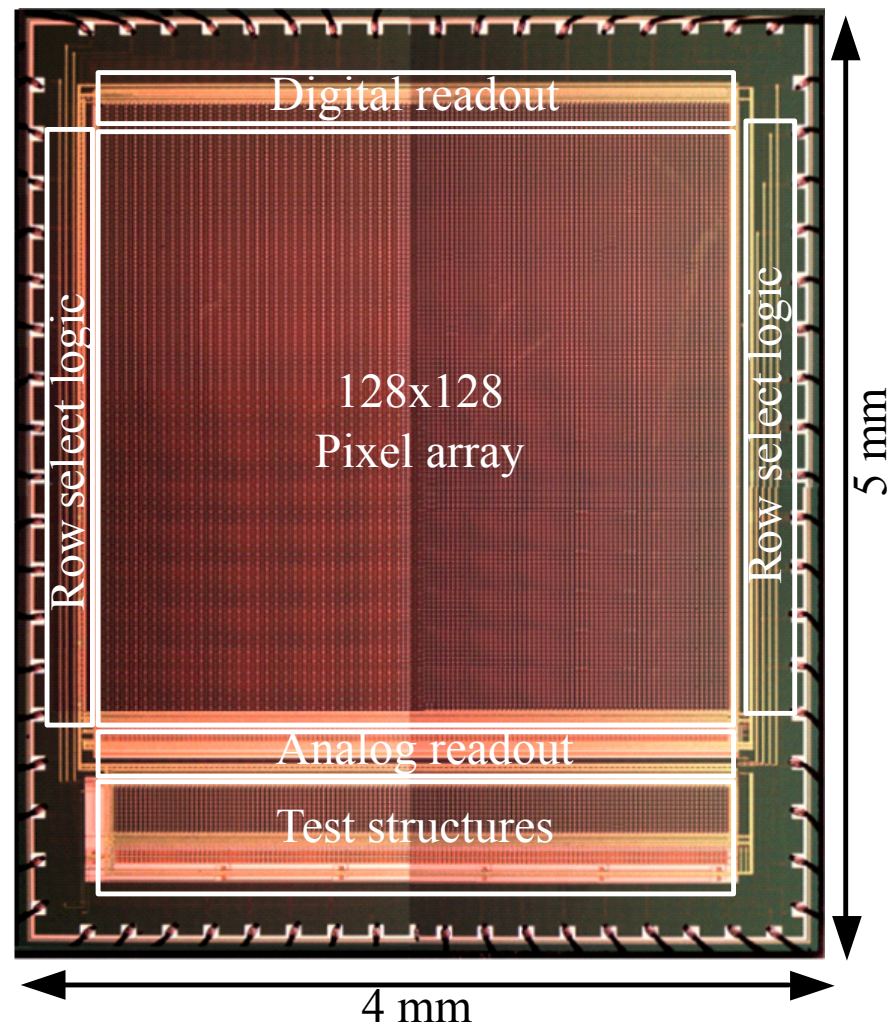

Figure 2.33: Die photograph of the sensor.

\subsubsection{Test setup}

Figure 2.34 shows the experimental platform used to characterize the image sensor. Two Xilinx Virtex-II pro development boards [2.20] are used to generate clocks and control signals for the image sensor test board, $A D C$ and frame grabber. Two FPGA boards are needed as the Virtex-II pro development boards have only 35 valid I/O pins for external interface, while over 50 valid I/O pins are needed for the entire test setup. An external $A D C$ is used to digitize the output signal of the image sensor. The $A D C$ used is a 12-bit $A D C$ from analog devices (AD9821) [2.21]. The frame grabber used in the measurement setup is an IC-PCI frame grabber installed in the PC.

DALSA's LABVIEW user interface is used to view the acquired image, the row and column waveform, histogram of each frame and average of the frame data. The mean and standard deviation of each row/column or frame is obtained 


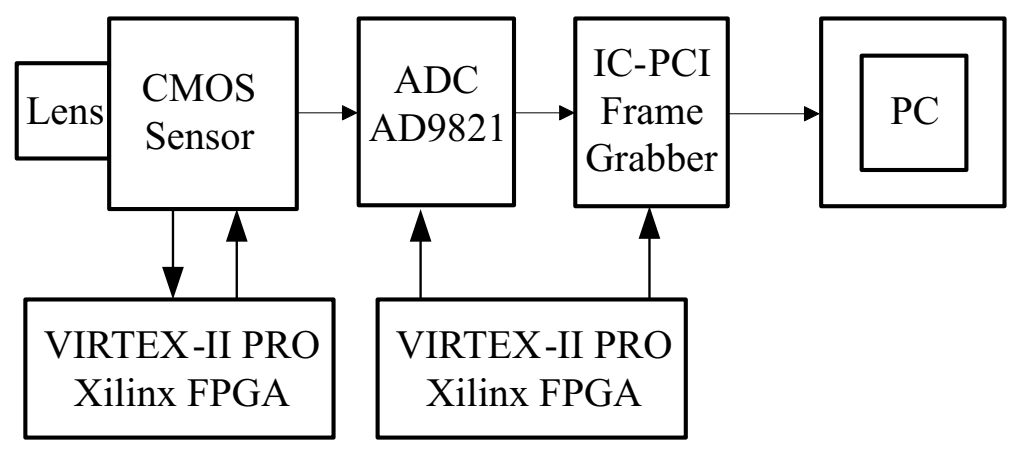

Figure 2.34: Image sensor characterization setup.

from this interface. The user interface also allows arithmetic operation like averaging and subtraction of pixels or frame data. The black level is an extra offset introduced in the pixel values. The user interface also provides controls to the frame grabber. The frame grabber can be operated in snap or grab mode. When the snap mode is selected, the existing image in the image window will be replaced by a new captured image. In grab mode the images are captured and displayed continuously.

For the experiments, an average measurement of 30 images was obtained to remove the random noise. All measurements were done at room temperature. If a pixel information is represented by $p(x, y)$ where $x$ and $y$ are the row and column number of the sensor array, then the average of a pixel over $N$ frames is given by

$$
p_{\text {avg }}(x, y)=\frac{1}{N} \sum_{n=1}^{N} p_{n}(x, y)
$$

A sensor array of size $X$ by $Y$ pixels is selected for most measurements, where $X$ and $Y$ are the total number of rows and columns respectively. The average of the array of pixels over $N$ frames is then computed as

$$
p_{\text {avg }}(X, Y)=\frac{1}{X Y N} \sum_{n=1}^{N} \sum_{x=0}^{X-1} \sum_{y=0}^{Y-1} p_{n}(x, y)
$$




\subsubsection{Measurements}

The image sensor has been tested and characterized and shown to be functional. A sample image of a projected text and human hand obtained from the image sensor with an integration time $20 \mathrm{~ms}$ is shown in the figure 2.35 .
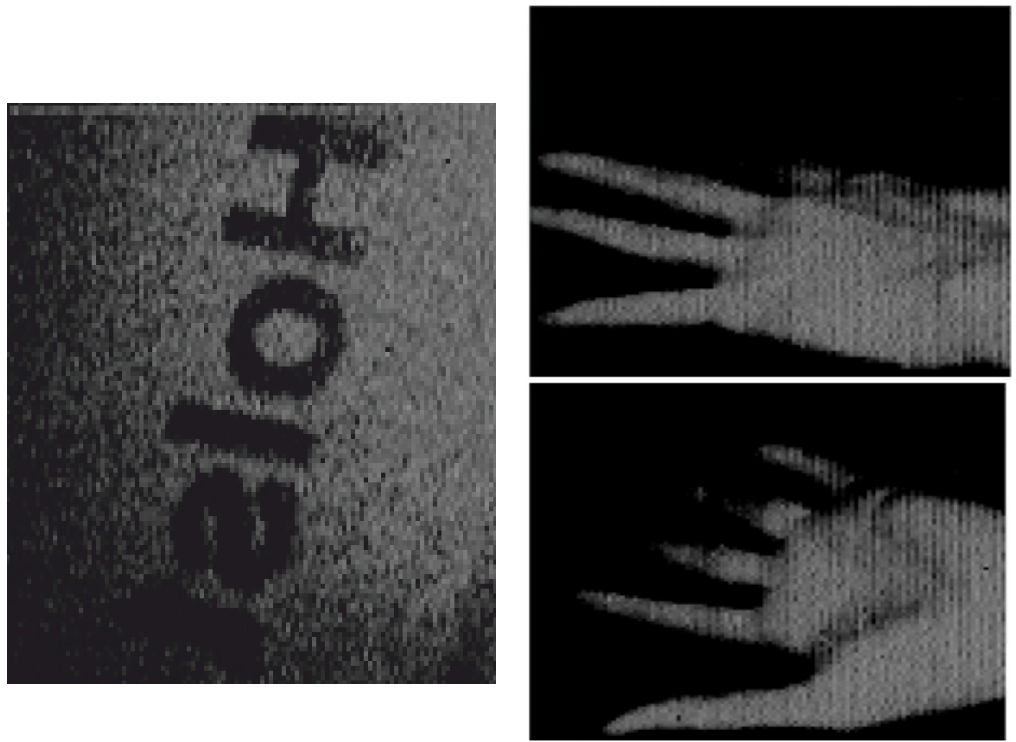

Figure 2.35: Sample image.

\subsubsection{Photo conversion characteristics}

The photo-conversion characteristics show the relationship between the output voltage and the incident photon flux. The incident photon flux is a function of the integration time. The photo-conversion characteristic for the image sensor when operated in delta differential sampling mode is shown in figure 2.36. The signal increases linearly before it reaches saturation. The measured maximum output voltage swing from the photo-conversion characteristics is $488 \mathrm{mV}$. This saturation voltage is limited by the column amplifier, which needs to drive the output voltage buffer in figure 2.27. The photosensitivity which refers to the ability to capture the desired details at a given scene illumination is obtained by dividing the slope of the photo conversion characteristics by the gain of the external $A D C\left(A_{A D C}\right)$. The digitally controlled variable gain amplifier of the $A D C$ is set to a digital code of 256 which implies an $A D C$ gain $\left(A_{A D C}\right)$ of 2.8. 
The sensitivity is thus calculated to be $13.94 \mathrm{mV} / \mathrm{ms}$ or $2.14 \mathrm{~V} / \mathrm{lux} . \mathrm{s}$.

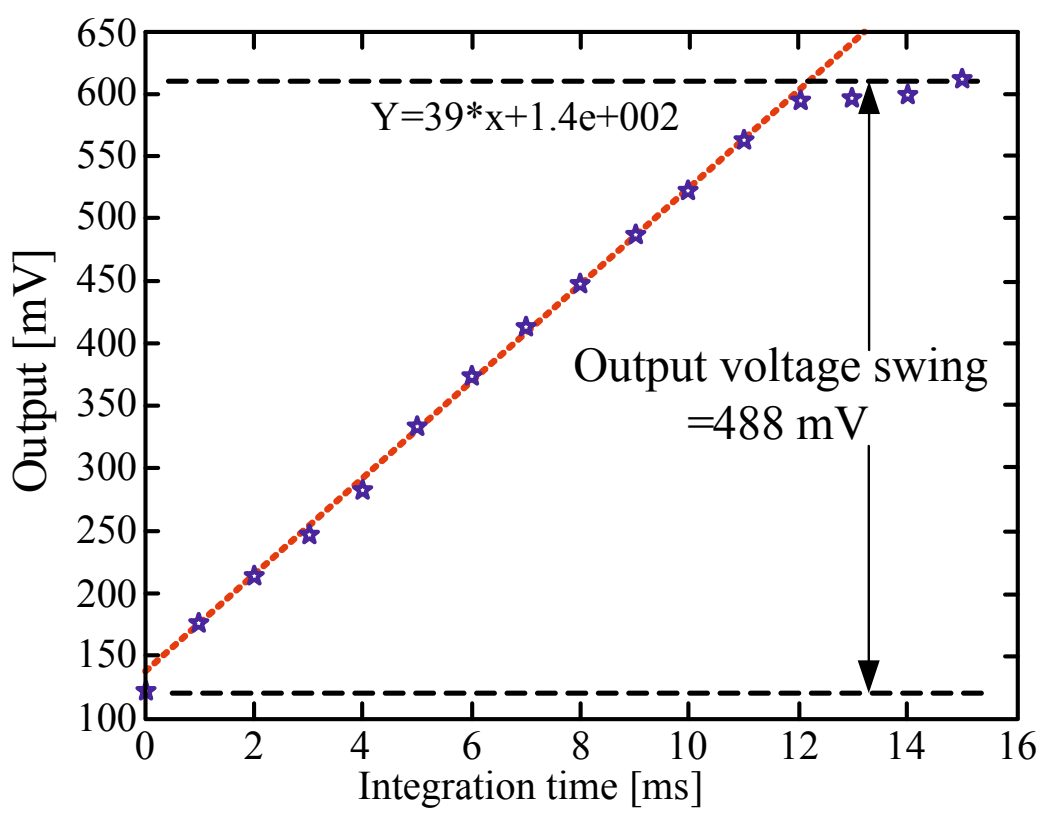

Figure 2.36: Photo conversion characteristics.

\subsubsection{Photon transfer curve PTC}

The photon transfer curve [2.22] is a very important method to calculate the various parameters and also to test the overall functionality of the pixel array of the sensor. The Photon Transfer Curve (PTC) is a log log plot of noise versus signal to cover the entire dynamic range of the imager. The standard deviation of the noise as a function of the average signal for a group of pixels contained in the array image is plotted.

For a typical array there are three distinct noise regimes. The first regime is the read noise floor measured under dark conditions. Read noise is the noise that is invariant of signal level and thus dominates at very low signal levels. The read noise is the random noise associated with the sensor output amplifier and its signal processing electronics and represents noise sources that are independent of the signal level. The noise floor (read noise) limits the image quality in the dark regions of an image and increases with exposure time due to the pixel dark current shot noise. As the illumination is increased, the dominating noise is the photon 
shot noise. This is the noise associated with the random arrival of the photons on the array and thus related to the input illumination. The process of random arrival of photons is governed by Poisson statistics, which means that the rms value of the shot noise is equal to the square root of the mean number of photons incident on a given pixel. Thus the shot noise profile becomes a straight line with a slope of $1 / 2$ on the $\log$-log curve. The third regime is associated with fixed pattern or pixel non-uniformity noise which results from sensitivity differences in each of the pixels. These differences are caused by the manufacturing process and are due to process variations, photo mask misalignments, etc. which cause each individual pixel to have a slightly difference sensitivity to the others pixel on the array. Pixel non-uniformity noise, also known as Photo Response NonUniformity or $P R N U$, is directly proportional to the signal level. The measured Photon Transfer Curve (PTC) is shown in figure 2.37.

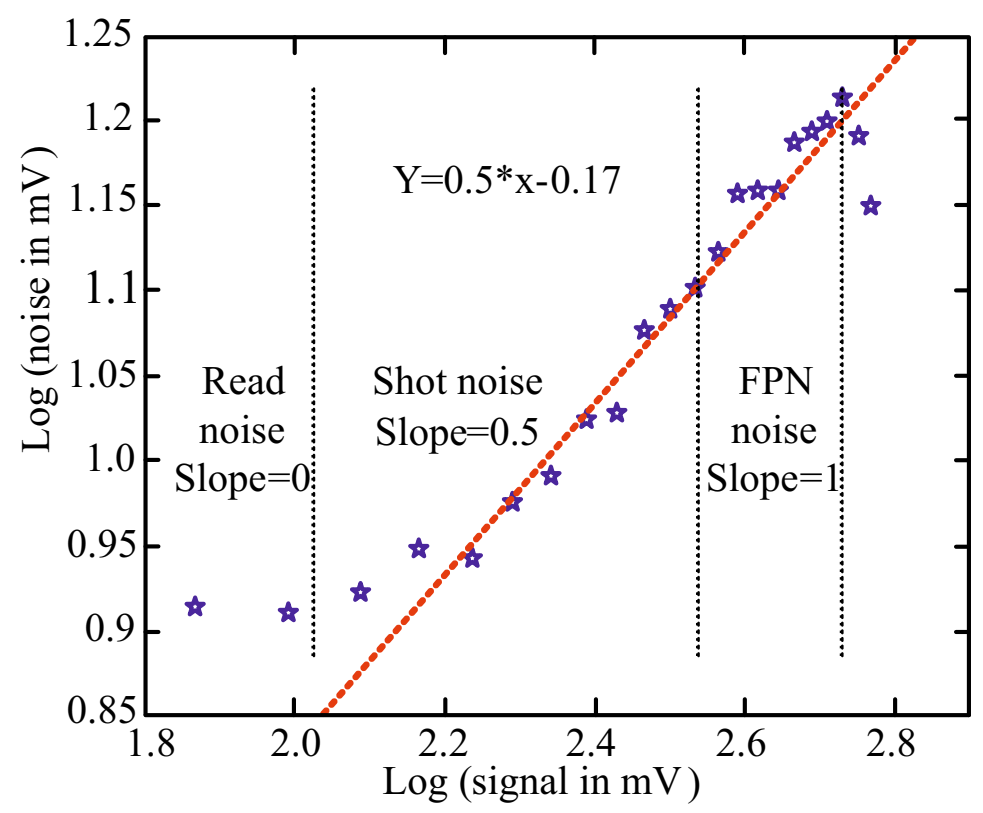

Figure 2.37: Photon transfer characteristics.

The conversion gain $(C G)$ of the designed image sensor is measured by extrapolating the photon shot noise back to the $\mathrm{X}$ axis intercept $(1 / 100.34$ in the case of figure 2.37) and dividing it by the sensor gain $\left(A_{S E N S O R}=A_{P I X E L}\right.$ x $A_{\text {OUTPUT }}$ x $\left.A_{A D C}\right)$. The measured conversion gain is $11.6 \mu \mathrm{V} / e^{-}$. The read noise shows the minimum detectable signal which is used for dynamic range 
calculations. The input referred noise of the pixel is measured to be $18 e^{-}$, which is the noise measured at the output of the $A D C$ divided by the sensor gain $\left(A_{S E N S O R}\right)$.

The reset noise is given by

$$
\overline{v_{\text {reset }}}=\sqrt{\frac{v_{T}}{C G}}
$$

where $v_{\text {reset }}$ is the reset noise, $v_{T}$ is the thermal voltage which is $26 \mathrm{mV}$ at room temperature of $27^{\circ} \mathrm{C}$. The reset noise $v_{\text {reset }}$ is calculated to be $47 e^{-}$. The reset noise is suppressed by the delta differential sampling operation.

The dynamic range of the sensor is determined by the full well capacity and the readout noise floor. The full well capacity is limited by the voltage swing and the charge storage capacitor. The capacitance at the sense node $\left(C_{F D}\right)$ is inversely proportional to $C G\left(C_{F D}=q / C G\right)$, where $q$ is the elementary charge. The calculated sense node capacitance is $14.5 \mathrm{fF}$. The full well or the saturation point of the pixel is the point where the PTC curve peaks. The full well from figure 2.34 is derived to be $43186 e^{-}$.

Dynamic range is an important figure of merit for image sensors. It expresses the ability to capture dark as well as bright sections within an image while maintaining the highest fidelity. For a linear image sensor, the dynamic range is the ratio of the largest possible signal divided by the smallest possible signal it can detect. The largest possible signal is directly proportional to the full well capacity of the pixel. The lowest signal is the noise level when the sensor is not exposed to any light, also called the "noise floor". Dynamic range $(D R)$ can be expressed as [2.23]

$$
\begin{aligned}
D R & =20 \log _{10} \frac{i_{\text {max }}}{i_{\text {min }}} \\
& =20 \log _{10} \frac{Q_{\text {sat }}-\left(i_{d c} \times t_{\text {int }}\right)}{\sqrt{\left(q \times i_{d c} \times t_{\text {int }}\right)+\sigma_{\text {read }}^{2}+\sigma_{\text {reset }}^{2}+\sigma_{F P N}^{2}}}
\end{aligned}
$$

where $Q_{s a t}$ is the well capacity, $i_{d c}$ is the dark current generated by the photodiode, $t_{\text {int }}$ is the integration time, $q$ is the elementary charge, $\sigma_{\text {read }}^{2}, \sigma_{\text {reset }}^{2}$ and $\sigma_{F P N}^{2}$ are the variances of the readout noise, reset noise and fixed pattern noise $(F P N)$ respectively. The overall dynamic range of the sensor is $45 \mathrm{~dB}$. At the sense node of the pixel, the input referred noise is $18 e^{-}$, which gives a dynamic range of $68 \mathrm{~dB}$. The dynamic range decreases for increasing integration time due 
to the effect of dark current.

Thermally generated charge, in addition to the photogenerated charge is accumulated over time at the sense node. This thermally generated current is known as dark current. The dark current charges the pixel capacitance even when there is no light on the pixel, limiting the sensor performance at low illuminations. The amount of dark charge produced is proportional to the integration time. The dark current is measured by shielding the image sensor from any light and acquiring a set of image data for different integration times. The rate of output change or the dark current measured is $18 \mathrm{pA} / \mathrm{cm}^{2}$ or $0.32 \mathrm{mV} / \mathrm{ms}$ at $27^{\circ} \mathrm{C}$.

The total power consumption measured is $100 \mathrm{~mW}$ at $30 \mathrm{fps}$. The high power consumption is due to the buffer amplifier/comparator inside the pixel which is used for in-pixel image processing. The buffer in the pixel consumes $4.14 \mu \mathrm{W}$, thus for the complete array the total consumption of all buffers is $67.8 \mathrm{~mW}$.

The measured performance parameters of the image sensor are summarized in table 2.3.

\begin{tabular}{|l|l|}
\hline Parameters & Measured \\
\hline Sensitivity & $13.94 \mathrm{mV} / \mathrm{ms}$ \\
\hline SNR & $33 \mathrm{~dB}$ \\
\hline Conversion gain & $11.3 \mu \mathrm{V} / e^{-}$ \\
\hline Read Noise (after CDS) & $18 e^{-}$at pixel level \\
\hline Dark Current @ 27 ${ }^{\circ} \mathrm{C}$ & $18 p \mathrm{~A} / \mathrm{cm}^{2}$ \\
\hline Dynamic Range (after CDS) & $68 \mathrm{~dB}$ at pixel level \\
\hline Sense Node Cap. & $14.5 \mathrm{fF}$ \\
\hline Full well & $43186 e^{-}$ \\
\hline
\end{tabular}

Table 2.3: Sensor performance characteristics

\subsection{Conclusions}

Any optical vision system consists of a micro-lens array and a CMOS image sensor. An microlens array with a field of view of $124^{\circ}$ and a maximum contrast of $0.3 \mathrm{LP} /{ }^{\circ}$ has been designed by $V U B$ as part of collaboration. The CMOS image sensor was designed using the $0.18 \mu \mathrm{m}$ CMOS CIS process from UMC. The pixel is a smart pixel with the ability to perform binarization, store both analog and binary data for current and previous frames. The sensor can be operated in either conventional single charge transfer mode or partial charge transfer mode. The sensor was characterized and was shown to be working. 


\subsection{References}

[2.1] M. Land and R. Fernald, "The evolution of eyes," Annual Review of Neuroscience, vol. 15, pp. 1-29, 1992.

[2.2] http://watchingtheworldwakeup.blogspot.com.

[2.3] M. Land and D. Nilsson, Animal Eyes. Oxford: Oxford University Press, 2002. ISBN:0198575645.

[2.4] P. Baker, C. Fermller, and Y. Aloimonos, "A spherical eye from multiple cameras (or how to make better models)," Proceedings of Conference on Computer Vision and Pattern Recognition, vol. 1, pp. 576-583, 2001.

[2.5] J. Duparre, P. Schreiber, P. Dannberg, T. Scharf, P. Pellia, R. Volkel, H. Herzig, and A. Brauer, "Artificial compound eyes - different concepts and their application to ultra flat image acquisition sensors," Proceedings of MOEMS and Miniaturized Systems IV SPIE, vol. 5346, pp. 89-100, 2004.

[2.6] T. Hessler, M. Rossi, J. Pedersen, M. Gale, M. Wegner, and H. Tiziani, "Microlens arrays with spatial variation of the optical functions," Digest of Topical Meeting on Microlens Array, vol. 13, pp. 42-47, 1997.

[2.7] H. C. Ko, M. Stoykovic, J. Song, V. Malyarchuk, W. M. Choi, C. Yu, J. G. III, J. Xiao, S.Wang, Y. Huang, and J. Rogers, "A hemispherical electronic eye camera based on compressible silicon optoelectronics," Nature, vol. 454, pp. 748-753, 2008.

[2.8] E. Adelson and J. Wang, "Single lens stereo with a plenoptic camera," IEEE Transaction on Pattern Analysis and Machine Intelligence, vol. 14, no. 2, pp. 99-106, 1992.

[2.9] J. Tanida and K. Yamada, "Thin observation module by bound optics (TOMBO): Concept and experimental verification," Applied Optics, vol. 40, pp. 1806-1813, 2001.

[2.10] K. Fife, A. E. Gamal, and H. Wong, "A 3d multi aperture image sensor architecture," Proceedings of Custom Integrated Circuits Conference, pp. 281-284, 2006.

[2.11] E. Moens, Y. Meuret, H. Ottevaere, M. Sarkar, D. S. S. Bello, P. Merken, and $\mathrm{H}$. Thienpont, "An insect eye-based image sensor with large field of view," Proceedings of SPIE, vol. 7716, pp. 7716-85, 2010. 
[2.12] A. Einstein, "Ueber eines die erzeuhung und verwandlung des lichtes betreffendedn heuristischen gesichtspunkt," Annalen der Physik, vol. 17, pp. 123-148, 1905.

[2.13] G. Weckler, "Operation of p-n junction photodetectors in a photon flux integrating mode," IEEE Journal of Solid-State Circuits, vol. 2, pp. 65-73, 1967.

[2.14] A. Gamal and H. Eltoukhy, "CMOS image sensors," IEEE Circuits and Device Magazine, pp. 6-20, 2005.

[2.15] B. Burkey, W. Chang, J. Littlehale, T. Lee, T. Tredwell, J. Lavine, and E. Trabka, "The pinned photodiode for an interline transfer CCD imager," Proceeding of International Electronics and Device Meeting, Technical Digest, pp. 28-31, 1984.

[2.16] N. Teranishi, A. Kohono, Y. Ishihara, E. Oda, and K. Arai, "No image lag photodiode structure in the interline CCD image sensor," Proceeding of International Electron Device Meeting, Technical Digest, pp. 324-3271, 1982.

[2.17] S. Decker, R. McGrath, K. Brehmer, and C. Sodini, "A 256 x 256 CMOS imaging array with wide dynamic range pixels and column-parallel digital output," IEEE Journal of Solid State Circuits, vol. 33, no. 12, pp. 20812091, 1998.

[2.18] E. Fossum, "CMOS active pixel image sensors," Nuclear Instruments and Methods in Physics Research, vol. 395, no. 12, pp. 291-297, 1997.

[2.19] R. Baker, CMOS Circuit Design, Layout, and Simulation. New York: IEEE Press, 2005. ISBN:047170055X.

[2.20] Xilinx Virtex-II Pro FPGA user guide. http://www.xilinx.com/support/documentation/user_guides/ug012.pdf.

[2.21] AD921:12-Bit 40MSPS ISP, Datasheet Rev0, 2002. http://www.analog.com/static/imported-files/Data_Sheets/AD9821.pdf.

[2.22] J. Janesick, Scientific charge coupled devices. SPIE Press, 2001. ISBN:9780819436986.

[2.23] X. Liu and A. E. Gamal, "Synthesis of high dynamic range motion blur free image from multiple captures," IEEE Transactions on Circuit and SystemsI, vol. 50, no. 4, pp. 530-539, 2003. 


\section{Design of a CMOS polarization sensor}

Compound eyes found in insects, besides performing their function in forming images and motion detection are also sensitive to other properties of light, i.e. the wavelengths or vector of skylight polarization. Polarization provides additional visual information than intensity and wavelength and also a more general description of light, and therefore it provides richer sets of descriptive physical constraints for the interpretation of the imaged scene.

In this chapter polarization of light waves will be discussed. The mechanism of polarization vision in the compound eyes of insects will also be discussed. Polarization vision is described in section 3.1 and a brief introduction to polarization of light is presented in section 3.2. Polarization vision in compound eyes and polarization cameras are presented in sections 3.3 and 3.4 respectively. The theory behind wire-grid polarizers is discussed in section 3.5 and a one dimensional wire-grid polarized implemented using standard CMOS metal wiring layers is presented in section 3.6. The performance characteristics of such a wire-grid polarizer are also presented in section 3.7. In the final section simulation studies using two dimensional metal layers to selectively transmit specific wavelengths are shown. 


\subsection{Polarization vision}

The three basic characteristics of light are intensity, wavelength or color and polarization. The intensity or brightness is an time-averaged expression of the amount of light emitted from the surface of the light source per unit area. The colors of the visible spectrum stretch from violet, with the shortest wavelength, to red, with the longest. Polarization is the plane of vibration of the electric field vector of light.

According to [3.1], "polarization vision" is the ability to discriminate between two monochromatic lights of the same intensity, but with a different angle of polarization and/or degree of linear polarization. Polarization vision is a generalization of intensity vision and analogous to color vision. Color vision is useful in segregating scenes and in recognition of objects while polarization vision discriminates reflectances from a scene of similar brightness [3.1]. The study of polarization is more general than that of intensity or color and can simplify some of the visual tasks (for example region and edge segmentation, material classification etc.) which are more complicated or practically infeasible when limited to intensity and color information. Because intensity is the compression of polarization information, a polarization camera can also function as a conventional intensity camera. The intensity vision methods can be implemented by such a camera either alone or together with polarization vision methods.

Practically all light that is reflected in most environments is partially polarized, which means that it can be represented by the sum of unpolarized states and completely linear polarized states. Even though the sun itself produces fully unpolarized light, partially polarized light is abundant in natural scenes [3.2]. The various forms of polarization in natural scenes are discussed in detail in section 3.2. In this thesis, the term "polarized light" will refer to partially polarized light.

Polarization-dependent optical systems generally fall into three classes:

a) systems which measure polarization properties and are therefore sensitive to any system-induced polarization variation,

b) systems which accurately measure intensities and are therefore sensitive to transmissions which change with polarization state, and

c) systems which route light based on its polarization state and are therefore sensitive to any system-induced polarization variation [3.3].

A polarization dependent optical system similar to type b), which measures the intensities varying with the polarization state, has been designed in this work. 


\subsection{Polarization of light - basics}

Light, viewed classically, can be considered as a transverse wave wherein the underlying oscillations of the electric and magnetic fields happen along directions perpendicular to the direction of propagation of the wave. Longitudinal waves, in contrast, have oscillations confined in the direction of the propagation as in sound waves. Polarization is a phenomenon peculiar to transverse waves. It is the distribution of the electric field in the plane normal to the propagation direction. An unpolarized or randomly polarized electromagnetic wave is one in which the orientation of the electric vector changes randomly.

The mathematical representation of a plane wave propagating in the $z$ direction is

$$
\vec{E}=E_{0} \cos \left(k z-\omega t+\varphi_{0}\right)
$$

The electric vector $\vec{E}$ may be decomposed into parallel $E_{x}$ and perpendicular $E_{y}$ components as

$$
\vec{E}=E_{x} \vec{x}+E_{y} \vec{y}
$$

where $E_{x}$ and $E_{y}$ are expressed in the form

$$
\begin{aligned}
& E_{x}=E_{x 0} \cos \left(k z-\omega t+\varphi_{x 0}\right) \\
& E_{y}=E_{y 0} \cos \left(k z-\omega t+\varphi_{y 0}\right)
\end{aligned}
$$

From equations (3.3) and (3.4) we have

$$
\begin{aligned}
\frac{E_{x}}{E_{x 0}} & =\cos (\zeta) \cos \left(\varphi_{x 0}\right)-\sin (\zeta) \sin \left(\varphi_{x 0}\right) \\
\frac{E_{y}}{E_{y 0}} & =\cos (\zeta) \cos \left(\varphi_{y 0}\right)-\sin (\zeta) \sin \left(\varphi_{y 0}\right)
\end{aligned}
$$

where $\zeta=k z-\omega t . \quad$ From equations (3.5) and (3.6) we get

$$
\left(\frac{E_{x}}{E_{x 0}}\right)^{2}+\left(\frac{E_{y}}{E_{y 0}}\right)^{2}-2\left(\frac{E_{x}}{E_{x 0}}\right)\left(\frac{E_{y}}{E_{y 0}}\right) \cos (\delta)=\sin ^{2}(\delta)
$$


where $\delta=\varphi_{x 0}-\varphi_{y 0}$ is called the phase shift.

Equation (3.7) defines an ellipse, thus the polarized electromagnetic wave of this form is known as elliptically polarized wave. The polarization ellipse is very useful since it allows the description of all states of a completely polarized light wave using a single equation. However this representation is inadequate because the light vector propagating in space traces an ellipse or any special form of an ellipse example, the circle or a straight line in a time interval of the order of $10^{-15} \mathrm{sec}$ which is very short to be able to trace the ellipse. Furthermore, the polarization ellipse only represents a completely polarized light and cannot be used to describe either unpolarized light or partially polarized light.

The circular form of the polarization ellipse can be obtained if the phase shift satisfies $\delta=n \pi / 2$, where $(\mathrm{n}=+/-1,+/-3 \ldots)$ and $E_{x 0}=E_{y 0}=E_{0}$. Then $\sin (\delta)=$ \pm 1 and $\cos (\delta)=0$, and thus equation (3.7) becomes

$$
E_{x}^{2}+E_{y}^{2}=E_{0}^{2}
$$

Equation (3.8) defines a circle, thus the polarized electromagnetic wave of this form is known as circularly polarized wave.

The linear form of the polarization ellipse is obtained if the phase shift $\delta=n \pi$, where $(\mathrm{n}=0,+/-1,+/-2 \ldots)$, then $\sin (\delta)=0$ and $\cos (\delta)= \pm 1$, thus equation (3.7) becomes

$$
\left(\frac{E_{x}}{E_{x 0}}+\frac{E_{y}}{E_{y 0}}\right)^{2}=0 \quad \text { or } \quad E_{y}= \pm \frac{E_{y 0}}{E_{x 0}} E_{x}
$$

Equation (3.9) defines a straight line, thus the polarized electromagnetic wave of this form is known as linearly polarized wave. A linearly polarized wave has its electric field vibrating in the same direction at all times at a particular point. A linearly polarized light is often referred to as plane polarized light or simply polarized light.

Linear polarization can be of two types, vertically or $s$-polarized ${ }^{1}$ and horizontally or $p$-polarized. A vertically polarized wave is one for which the electric field lies only in the $x$ - $z$ plane as shown in figure 3.1(a). Horizontally polarized wave is one for which the electric field lies only in the $y-z$ plane as shown in figure 3.1(b).

Elliptically and circularly polarized light is rare in nature [3.4] and thus the discussion will be limited to the detection and analysis of linear polarization in

\footnotetext{
${ }^{1}$ The name $s$-polarized comes from German senkrecht meaning perpendicular and $p$ is parallel polarized with respect to the plane of incidence.
} 

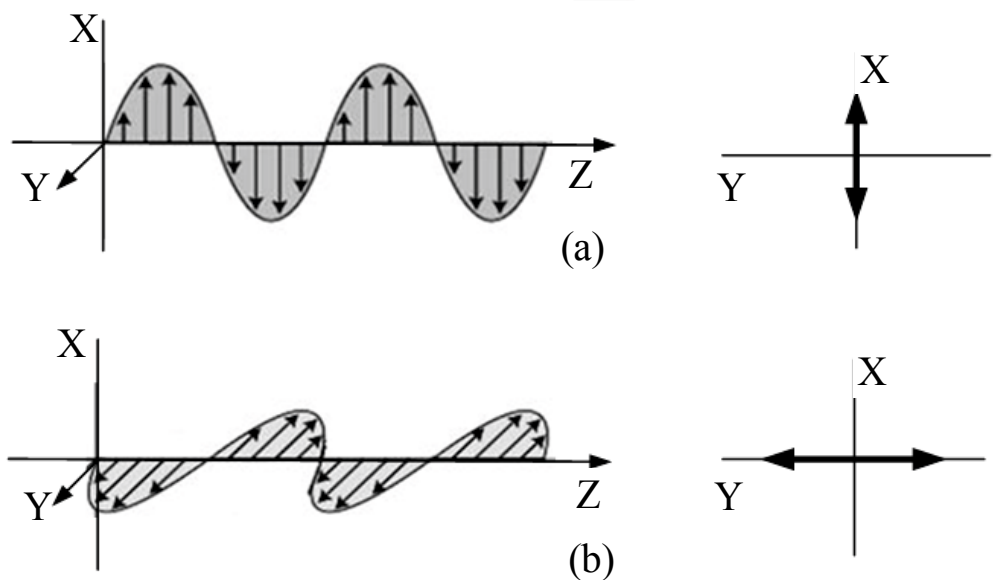

Figure 3.1: Linear polarization, (a) s-polarized wave (b) p-polarized wave.

this thesis.

\subsubsection{Polarization of light from an unpolarized beam}

A linearly polarized beam is obtained from an unpolarized beam by removing all waves from the beam except those whose electric field vectors oscillate in a single plane. A polarized light from an unpolarized light can be achieved through a linear polarizer either by the phenomenon of absorption, reflection, refraction or scattering.

\subsubsection{Polarization by absorption}

The most common technique for producing polarized light is by passing the unpolarized light through a material that absorbs electric field vibrating in all directions except the vibrations in a plane parallel to a certain direction. Such a device is also called a polarizer. One such type of polarizing filter was invented by Edwin H. Land in 1928 and was called Polaroid. A Polaroid consists of long-chain organic molecules that are aligned in one direction, placed in a plastic sheet. They form a closely spaced linear grid, which allows the passage of light vibrating only in the same direction as the grid while absorbing the vibrations in other direction as shown in figure 3.2. 


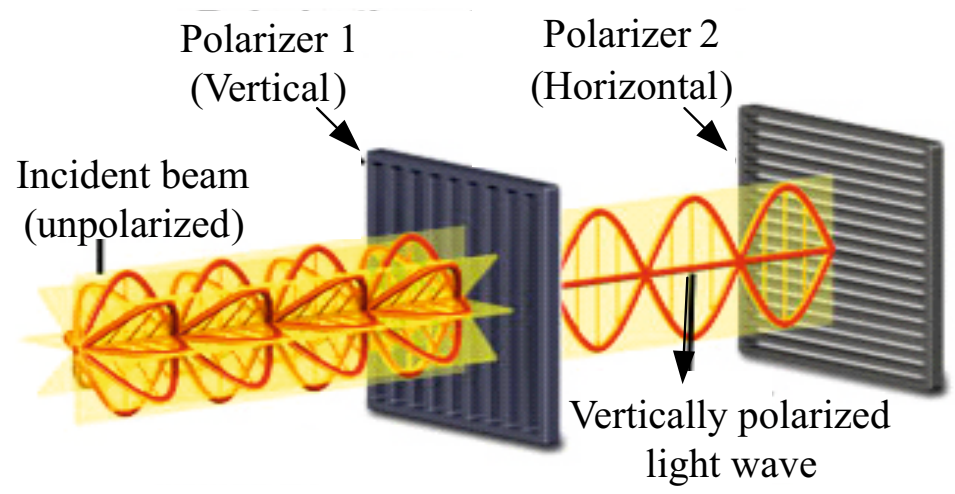

Figure 3.2: Polarization by absorption [3.5].

\subsubsection{Polarization by reflection}

When an unpolarized light beam is reflected from a surface, the reflected light may be completely polarized, partially polarized, or unpolarized, depending on the angle of incidence. For an angle of incidence of $0^{\circ}$, the reflected light is unpolarized, while for other values of angle of incidence, the reflected light is polarized with a vibration direction parallel to the reflecting surface as shown in figure 3.3. If part of the incident light enters the material, the refracted light will also be polarized with a vibration direction perpendicular to the path of the refracted ray.

\subsubsection{Polarization by refraction}

Optically anisotropic materials have different optical properties in different directions. Double refraction is an optical property where a single ray of unpolarized light entering an anisotropic medium is split into two rays, each travelling in different direction as shown in figure 3.4. Such materials with different indices of refraction associated with different crystallographic directions are known as birefringent materials. Birefringent uni-axial crystalline materials have a unique axis of symmetry, known as the optic axis where no double refraction occurs. The propagation of light along the optic axis would be independent of its polarization; its electric field is everywhere perpendicular to the optic axis and it is called the ordinary- or $O$-ray. The light wave with its electric field parallel to the optic axis is called the extraordinary- or $E$-ray. When an unpolarized light enters a birefringent crystal, the ordinary wave will 


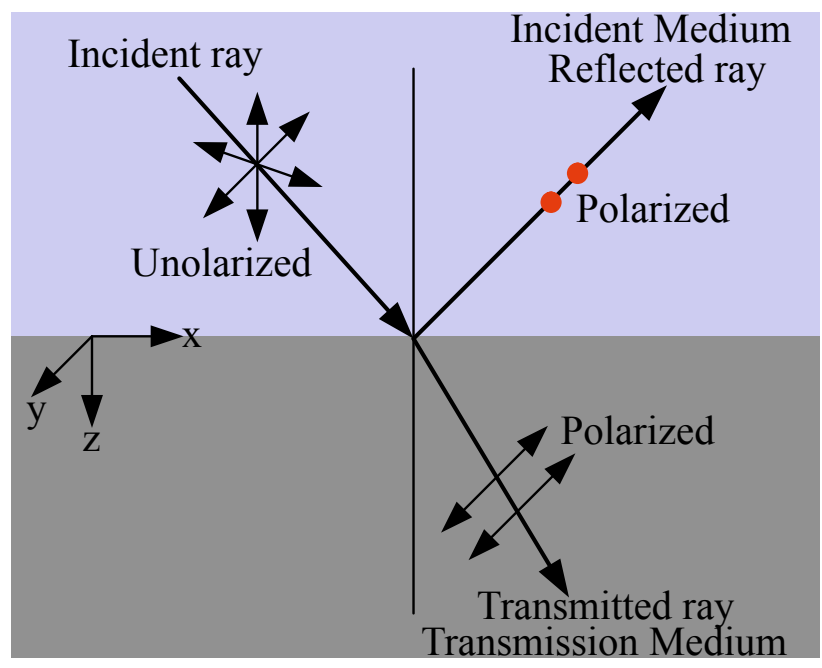

Figure 3.3: Polarization by reflection.

pass straight through, while the extraordinary wave is displaced by a distance depending on the thickness of the crystal and the angle of refraction.

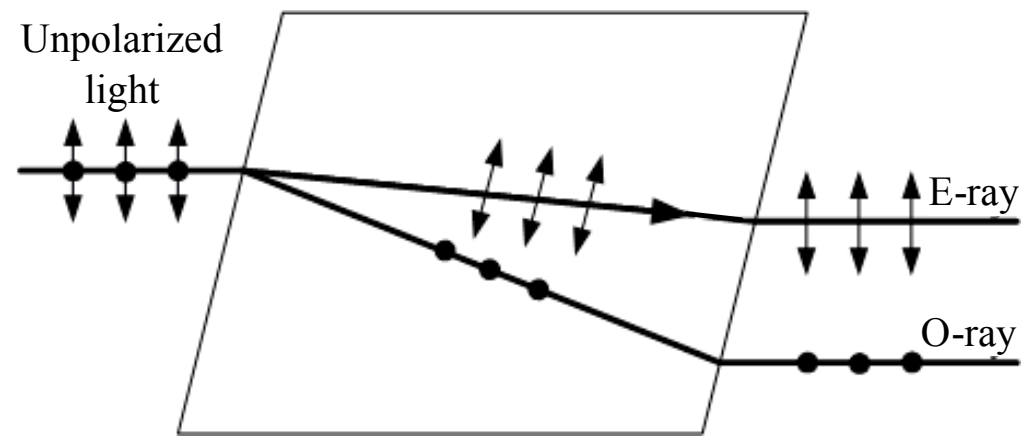

Figure 3.4: Polarization by double refraction.

\subsubsection{Polarization by scattering}

Polarization or depolarization also occurs when light is scattered while traveling through a medium. When light strikes the atoms of a material, it often sets the electrons of those atoms into vibration. The vibrating electrons then produce their 
own electromagnetic wave which is radiated outward in all directions. This newly generated wave strikes neighboring atoms, forcing their electrons into vibrations at the same original frequency. These vibrating electrons produce another electromagnetic wave which is once more radiated outward in all directions. This absorption and re-emission of light waves causes the light to be scattered about the medium. This scattered light is partially polarized.

\subsubsection{Polarization of light - representation}

The state of the polarization as seen from equation (3.7) can be completely defined by four parameters: two amplitudes, the magnitude and the sign of the phase shift. The phase differences are difficult to measure and are not additive when many independent light waves are mixed. For this reason, Stokes parameters introduced in 1852 by Sir George Gabriel Stokes [3.6] are often used to define the state of the polarization. They provide a phenomenological description of the polarization state of light, involving only the optical intensity that can be experimentally determined. Poincaré sphere was developed by Henri Poincaré in 1892 [3.7], and is used to visualize the orientation and elongation of the polarization ellipse. In this section, the Stokes parameters are related to intensity and the orientation angles of the Poincare sphere.

The polarization ellipse discussed in section 3.2 describes the polarized light in terms of its amplitude. The amplitude of the optical field cannot be observed, but it is possible to observe and measure its intensity, which is equal to the time average of the square of the field amplitudes. This can be done by taking a time average of the time dependent quantities in equation (3.7). The time average of the field components are defined by the following equation:

$$
\left\langle E_{x}(z, t) E_{y}(z, t)\right\rangle=\lim _{T \rightarrow \infty}\left(\frac{1}{T} \int_{0}^{\infty} E_{x}(z, t) E_{y}(z, t) d t\right)
$$

where $T$ is the time of the measurement. To evaluate equation (3.7) the denominator is first removed by multiplying by the factor $4 E_{x 0}{ }^{2} E_{y 0}{ }^{2}$. We then have

$$
\begin{aligned}
& 4 E_{y 0}{ }^{2}\left\langle E_{x}^{2}(z, t)\right\rangle+4 E_{x 0}^{2}\left\langle E_{y}^{2}(z, t)\right\rangle \\
& \quad-8 E_{x 0} E_{y 0}\left\langle E_{x}(z, t) E_{y}(z, t)\right\rangle \cos (\delta)=\left(2 E_{x 0} E_{y 0} \sin \delta\right)^{2}
\end{aligned}
$$


Using equations (3.3), (3.4) and (3.10), the time averages in equation (3.11) are found to be

$$
\begin{aligned}
\left\langle E_{x}{ }^{2}(z, t)\right\rangle & =\frac{1}{2} E_{x 0}{ }^{2} \\
\left\langle E_{y}{ }^{2}(z, t)\right\rangle & =\frac{1}{2} E_{y 0}{ }^{2} \\
\left\langle E_{x}{ }^{2}(z, t) E_{y}{ }^{2}(z, t)\right\rangle & =\frac{1}{2} E_{x 0} E_{y 0} \cos \delta
\end{aligned}
$$

Substituting equation (3.12) in (3.11) and rearranging we get

$$
\begin{aligned}
\left(E_{x 0}{ }^{2}+E_{y 0}\right)^{2}-\left(E_{x 0}{ }^{2}-E_{y 0}{ }^{2}\right)^{2} & \\
& -\left(2 E_{x 0} E_{y 0} \cos \delta\right)^{2}=\left(2 E_{x 0} E_{y 0} \sin \delta\right)^{2}
\end{aligned}
$$

From equation (3.13) we can define

$$
S_{0}^{2}=S_{1}^{2}+S_{2}^{2}+S_{3}^{2}
$$

where

$$
\begin{aligned}
S_{0} & =E_{x 0}{ }^{2}+E_{y 0}{ }^{2} \\
S_{1} & =E_{x 0}{ }^{2}-E_{y 0}{ }^{2} \\
S_{2} & =2 E_{x 0} E_{y 0} \cos \delta \\
S_{3} & =2 E_{x 0} E_{y 0} \sin \delta
\end{aligned}
$$

The parameters $S_{0}, S_{1}, S_{2}$ and $S_{3}$ are known as the Stokes polarization parameters for a plane wave.

The first parameter expresses the total intensity of the optical field while the remaining three parameters describe the polarization state of light. The Stokes vector helps in the measurement of

i.) the intensity of light

ii.) the degree of linear polarization with respect to vertical and horizontal axes

iii.) the degree of linear polarization with respect to the axes oriented at $+45^{\circ}$ and $-45^{\circ}$

iv.) the degree of left and right circular polarizations 
One of the main advantages of the Stokes formalism is that it allows for the determination of the degree of polarization $(D O P)$ of any optical signal directly from its Stokes components. In most cases, an electromagnetic wave consists of the incoherent superposition of a fully polarized wave and of a fully unpolarized wave. The $D O P$ quantifies that fraction of the total optical signal that is actually polarized, with:

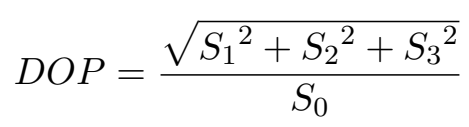

The $D O P$ of a fully polarized optical signal is equal to one, whereas the $D O P$ of an unpolarized optical signal is zero. In a linearly polarized light beam,the circular and elliptical polarizations are zero. Its degree of polarization is thus often referred to as degree of linear polarization $(D O L P)$. The degree of linear polarization of a light beam is defined by:

$$
D O L P=\frac{S_{1}}{S_{0}}
$$

To visualize the polarization ellipse of a propagating polarized light wave characterized by an azimuthal angle and an ellipticity angle, Poincaré sphere as shown in figure 3.5 is used. On the sphere, the longitude lines represent the ellipticity angle $\chi$. and the latitude lines represent the azimuthal angle $\psi$. The Stokes parameters are the Cartesian coordinates of a space in which any completely polarized light beam is represented by a point on a sphere with unit radius around the origin.

The Cartesian coordinates $(x, y, z)$ of the Stokes parameters are mapped to the spherical coordinates $(I, \psi, \chi)$ of the Poincaré sphere [3.8] of unit radius using the following equations

$$
\begin{aligned}
& S_{1}=\delta \cos (2 \psi) \cos (2 \chi) \\
& S_{2}=\delta \sin (2 \psi) \cos (2 \chi) \\
& S_{3}=\delta \sin (2 \chi)
\end{aligned}
$$

The Stokes parameter $S_{0}$ represents the radius of the Poincare sphere, while $S_{1}$, $S_{2}$ and $S_{3}$ represent the coordinates of a point on the surface of the sphere. Each point on the surface of the sphere corresponds to a state of the polarization of the light. Right circular polarization is represented at the north pole and left circular 


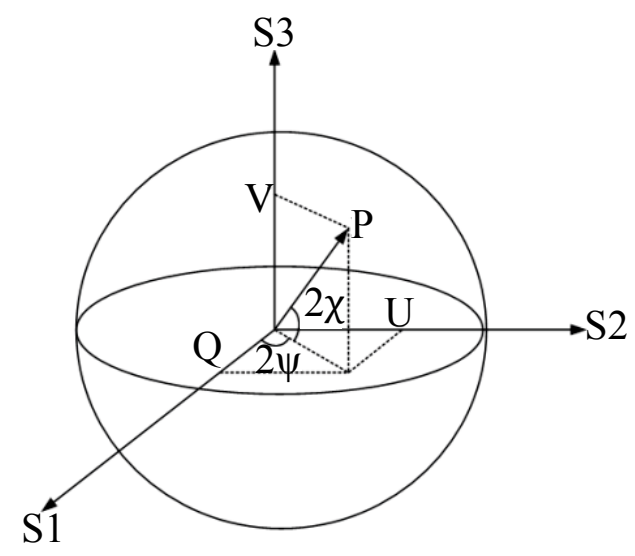

Figure 3.5: Poincaré sphere representation.

polarization at the south pole of the sphere. Linear polarization is represented along the equator of the sphere. Elliptical polarization of all possible azimutal and ellipticity angles are represented by other points on the surface of the sphere. The polarization azimuth angle $\psi$ and the ellipticity angle $\chi$ can then be derived from equation (3.18) as:

$$
\begin{aligned}
\sin (2 \chi) & =\frac{S_{3}}{\sqrt{S_{1}^{2}+S_{2}^{2}+S_{3}^{2}}} \\
\tan (2 \chi) & =\frac{S_{2}}{S_{1}}
\end{aligned}
$$

The parameter $\chi$ varies from $+45^{\circ}$ to $-45^{\circ}$. It is positive for right-handed polarization forms and negative for left-handed polarization forms. The parameter $\psi$ varies from $0^{\circ}$ to $180^{\circ}$, it is $0^{\circ}$ for parallel polarization and $90^{\circ}$ for perpendicular polarization.

\subsection{Polarization vision in compound eyes}

The most important use of the sophisticated eyes of many higher vertebrates and many invertebrates are in image formation and motion detection. They are however also able to detect the other properties of light such as the wavelength (color) or the vector of polarization. The sensitivity to polarization has been shown to be useful in a number of visual functions including increase in contrast, orientation, navigation, prey detection, predator avoidance and intra-species 
signaling.

It was shown in chapter 2 that the compound eyes of insects are formed by hundreds or thousands of simple eyes called ommatidia. Each of these subunits has its own lens, crystalline cone, and several long visual cells arranged in a star pattern. The light sensitive parts of the visual cells are the microvilli which are an array of tube-like membranes where the pigment rhodopsin is located. In all animals, invertebrates and vertebrates, the visual pigment rhodopsin is present in the photoreceptor membrane of the visual cells. These photoreceptors are preferentially sensitive to a specific $e$-vector direction due to their strong dipole moment. They are maximally excited when the electrical vector (e-vector) axis is parallel to the dipole axis. In vertebrates, these photoreceptors are oriented perpendicular to the paths of the incoming light rays, presenting a random array and thus are typically insensitive to polarized light. In invertebrates, the photoreceptors are aligned parallel to the microvillar axis. Thus if all microvilli of a single photoreceptor cell are parallel, the cell will respond most strongly to incoming polarized light with its $e$-vector aligned parallel to the microvillus, thus exhibit polarization sensitive behavior [3.9].

The detection of polarization in the eyes of insects is mediated through the ommatidia present in the dorsal rim area $(D R A)$ of the compound eye. In each ommatidium, the photoreceptors come in two sets that have their microvilli oriented at about $90^{\circ}$ to each other. The receptors are thus tuned to mutually orthogonal $e$-vectors promoting polarization antagonism [3.10]. The high polarization sensitivity of the DRA is attributed to the microvilli being aligned along the rhabdomere. The polarization sensitivity of the ommatidia outside the DRA of the compound eye is weak, because of the misalignment of the microvilli [3.10]. The variations in the ommatidia in the DRA and outside of the compound eye of the field cricket are shown in figure 3.6.

\subsection{Polarization cameras}

The polarization state of light can be easily visualized using polarization filters. A polarization filter has the property to either transmit light vibrating in one direction producing linearly polarized light or light with a sense of rotation producing circularly polarized light. Basically a polarization filter transforms polarization information into intensity difference information. This is important because humans are able to perceive intensity information but not polarization information.

A polarization camera is a generalization of the conventional intensity camera. 


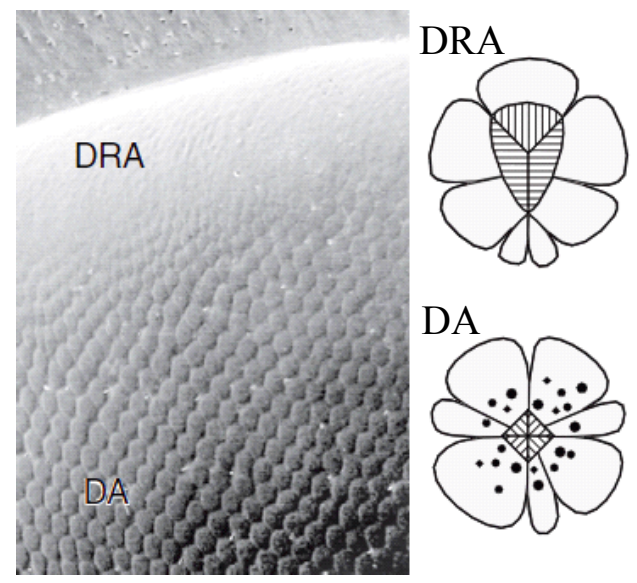

Figure 3.6: Cross section of ommatidia in the DRA and outside DRA (DA) regions of the compound eye of the field cricket in the dorsal most part of the compound eye [3.10].

To obtain parameters describing the polarization state of the monochromatic light, a set of measurements has to be taken from different positions of the polarizers, waveplates and photodetectors. Therefore, data acquisition time depends on how fast the polarizers, waveplates and photodetectors are moved to their various measurement positions. In addition, the precision of positioning the polarizers, waveplates and photodetectors influences the results of measurements.

State of the art polarization image sensors consist of a photodetector array, such as a CMOS or a CCD sensor array, and a single or multiaxis micropolarizer array to measure the polarization information in real time. Based on the position of the micropolarizers and the mode of data acquisition, the current state-ofthe-art polarization image sensors can be divided into two types: time domain multiplexed mode sensors and spatial domain multiplexed mode sensors.

The first group of polarization sensors includes standard imaging sensors coupled with electrically or mechanically controlled polarization filters [3.11]. It is operated in time domain multiplexed mode, where in, the externally coupled linear polarizer is rotated in front of either a single photodetector or a complete photodetector array. The main disadvantages these systems is that a mechanical or electrical rotation of the polarizer filter is required, thus it is not suitable for real time design. Multiple images with different orientation of the polarization filter are required for the measurement of partial linear polarization, increasing the frame time by the total number of orientation measurements desired. Further as the precision of the position of the polarizers effects the measurement results, 
these systems often produces erroneous results. However these systems have higher spatial resolution as the whole imaging array images only one orientation at one time.

The second group of polarization sensors includes image sensors where the linear micro-polarizer is either fabricated directly on the sensor array or on a substrate and then flip-chip bonded with the sensor array. Monolithic integration of micropolarization filters with CMOS imaging sensors is currently a subject of intense research. A plane coated with micrometer-scale polarizing elements in a variety of orientations is called a micropolarizer array. They operate in spatial domain multiplexed mode where, the linear polarizers are oriented in different directions with respect to the photodiodes and are directly fabricated on top of the photodiode. Micropolarizers have been fabricated with metal grids and organic materials. Incorporating pixel-pitch-matched polarization filters at the focal plane for visible spectrum has been explored with birefringent materials [3.12] and thinfilm polarizers [3.13], [3.14].

Photolithographically patterning polymer polarization film to make micropolarizer arrays was proposed by Faris [3.15]. Polymer film micropolarizer arrays have been successfully deployed in display applications, but their use in imaging is more challenging. For imaging, the thickness of the polarizing element has to be much smaller than the pixel size to have a large light collection angle. Polymer polarizers are made by mechanically stretching the polymer film. It is difficult to manufacture uniform stretched films with a thickness of less than $10 \mu \mathrm{m}$ [3.16]. Another main disadvantage of the thinfilm polymer polarization imaging sensors has been the complex fabrication techniques required to merge CMOS technology with polymer filters. These fabrication steps are prone to misalignment errors, which can degrade the sensing capabilities of the polarization imaging sensor. Furthermore, the thick multilayer filter array, which is around 10 micron of thickness, is prone to optical crosstalk and limits the extinction ratios of the sensors [3.17]. The spatial orientation of the linear polarizer filter results in reduced spatial resolution. However since the linear polarizers are directly incorporated on top of the photodiode, the temporal resolution is increased allowing for faster polarization imaging and also contributes to the compactness of the design. The precision of the system also increases as the position of the polarizer is fixed.

Metal-grid polarizers for electromagnetic radiation have been used for a long time and can be dated back to Hertz [3.18]. Metal-grid micropolarizer arrays are often used for infrared imaging polarimetry [3.19], but they were not very effective in the visible wavelength range. However with the scaling of the modern 
CMOS processes the metal planes normally used for interconnections when specifically aligned are now becoming suitable for polarization transmission [3.20], [3.21]. Wire grid polarizers are discussed in detail in section 3.5.

Table 3.1 summarizes the discussion on different types of linear micropolarizers.

\begin{tabular}{|l|l|l|l|}
\hline & External filter & $\begin{array}{l}\text { Organic micro- } \\
\text { polarizer }\end{array}$ & $\begin{array}{l}\text { Metallic wire } \\
\text { grid polarizer }\end{array}$ \\
\hline Operating mode & Time domain & Spatial domain & Spatial domain \\
\hline $\begin{array}{l}\text { Mechanical } \\
\text { intervention }\end{array}$ & Required & Not required & Not required \\
\hline Extinction ratio & Very high & High & Low \\
\hline Spatial resolution & High & Low & Low \\
\hline Temporal resolution & Low & High & High \\
\hline $\begin{array}{l}\text { Additional fabrication } \\
\text { required? }\end{array}$ & $\begin{array}{l}\text { No (externally } \\
\text { coupled) }\end{array}$ & Yes & $\begin{array}{l}\text { No (Standard } \\
\text { CMOS metal } \\
\text { layers are used) }\end{array}$ \\
\hline
\end{tabular}

Table 3.1: Comparison of linear polarizers.

\subsection{Wire-grid polarizer}

A wire-grid is used to denote a planar structure composed of a series of parallel wires or strips made of a conducting material. Wire-grid polarizers are inorganic optical polarization devices made of aluminum. Aluminum is usually chosen for the wire-grid polarizer in the visible region of the electromagnetic spectrum as it is highly reflective and absorbs only a small portion of the incident electromagnetic wave in the visible region. Furthermore they are able to withstand high temperatures, while the performance of organic polarizers is typically degraded at high temperatures [3.22]. The aluminum wire-grid polarizer also provides high transmission efficiency and polarization extinction ratio.

Normally, such a structure would function as a simple diffraction grating, but when the pitch or period of the wires is less than half the wavelength of the incoming light, it becomes a polarizer. Wire-grid polarizers are extensively used as polarization sensitive elements in various applications because of their excellent polarization performance and planar structure that allows them to be easily pixilated and integrated into other optoelectronics devices. A schematic view of a wire-grid polarizer is shown in figure 3.7. 


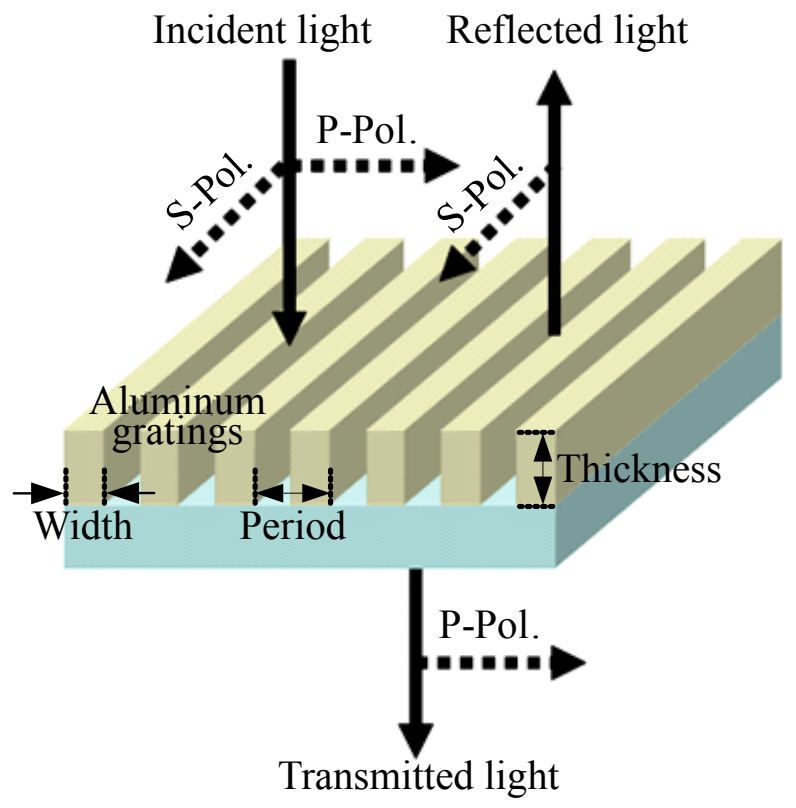

Figure 3.7: Wire-grid polarizer.

The polarization effects that occur when an electromagnetic wave is transmitted through a wire-grid having a grid pitch smaller than the wavelength of the incident electromagnetic wave was first observed by Heinrich Hertz in 1888 [3.18]. When light is incident on the wire-grid polarizer, the light which passes through the grating structure is affected depending on the size, shape and spacing of the grating structure. The wire-grid polarizer transmits electromagnetic waves whose electric field is perpendicular to the grid wires ( $T M$ polarization) and reflects or radiates off the electromagnetic waves with an electric field parallel to the wires (TE polarization). The wire-grid polarizer acts like a mirror for reflected $s$-plane polarized light and as a dielectric for the $p$-plane polarized light as shown in the figure 3.7. For the one dimensional grating shown in figure 3.7, appropriate selection of dimensions of the wire-grid will result in behavior as a polarizer, waveplate or polarization dependent filter.

The theoretical explanation for the behavior observed for the transmission of the electromagnetic wave through a wire-grid polarizer was provided by Horace Lamb in 1898 [3.23]. For a grating of strips, the transmission coefficients were 
shown to be

$$
\begin{aligned}
& T_{\|}=\frac{y^{2}}{1+y^{2}} ; y=\frac{2 D}{\lambda} \ln \left[\sec \pi\left(\frac{1}{2}-\frac{A}{D}\right)\right] \\
& T_{\perp}=\frac{1}{1+x^{2}} ; x=\frac{2 D}{\lambda} \ln \left[\sec \left(\frac{\pi A}{D}\right)\right]
\end{aligned}
$$

where $A$ is the half-width of the strip, $D$ is the grating space, $\lambda$ is the wavelength of the incident light, $T_{\|}$and $T_{\perp}$ are the transmission coefficients of the electric field parallel and perpendicular to the grid wires respectively. The equation shows the dependence of the transmitted intensity on the size of the wire-grid. The equations also show that for $\lambda \gg D$, the transmission coefficient of the electric field parallel to the wire-grid is nearly zero; thus the transmitted electric field is completely polarized with only the orthogonal electric field present.

The wire-grid polarizer structure acts as a diffraction grating whose interaction with incident light can be modeled by rigorous application of the diffraction grating theory and the boundary conditions of Maxwell's equations. If $t$ is the grating width and a is the grating period $(t+D)$, the refractive indices of the $T E$ wave $n_{t e}$ (the electric vector parallel to the grating grooves) and the TM wave $n_{t m}$ (the electric field vector normal to the grating groove) are expressed as:

$$
\begin{aligned}
& n_{t e}{ }^{2}=R n_{1}{ }^{2}+(1-R) n_{2}{ }^{2} \\
& n_{t m}{ }^{2}=\frac{n_{1}{ }^{2} n_{2}{ }^{2}}{R n_{2}{ }^{2}+(1-R) n_{1}{ }^{2}}
\end{aligned}
$$

where $n_{1}$ is the dielectric constant of the grating material, $n_{2}$ is the dielectric constant of the fill material and $R$ is the ratio of the grating width to the grating period $R=t / a$.

The key parameters that determine the performance of a wire-grid polarizer are its period (pitch), linewidth, and depth of the grid elements.

The depth of the grid elements (thickness of aluminum) should be enough to be optically opaque and it should also be able to achieve the desired transmission and extinction ratio. The wire height of a visible spectrum wire-grid polarizer is typically between $100 \mathrm{~nm}$ and $200 \mathrm{~nm}$. The wire-grid polarizer aspect ratio is the ratio of the wire thickness to the width of the wire. For a visible spectrum wiregrid polarizer the aspect ratios usually range from $2: 1$ to $25: 1$. The linewidth is the width of the polarizer material. Typically the linewidth for visible spectrum 
wire-grid polarizers is approximately $60 \mathrm{~nm}$, although this may vary based upon the desired performance of the polarizer. The duty cycle is defined as the ratio of line width and the pitch. It is basically the amount of space one wire occupies compared to the pitch. The duty cycle range generally used to manufacture visible spectrum wire-grid polarizers is from $40 \%$ to $60 \%$. The wire-grid polarizer duty cycle affects the reflection and transmission efficiencies as well as the reflection and transmission extinction ratios.

Pitch (period) is defined as the distance between the center of two consecutive polarizer wires. The wavelength for which a given grid will act as a useful polarizer is $\lambda=2 d$, where $d$ is the grid spacing [3.24]. The pitch needs to be at least three times smaller than the smallest wavelength that is being polarized for optimum performance. For example, to efficiently polarize a spectrum with a minimum wavelength of $400 \mathrm{~nm}$, the wire-grid polarizer should have a pitch of at most $133 \mathrm{~nm}$.

Standard CMOS processes use metal layers to connect circuit elements. The cross-sectional view of the metal layers of the standard CMOS process technology from $0.18 \mu \mathrm{m}$ UMC is shown in the figure 3.8.

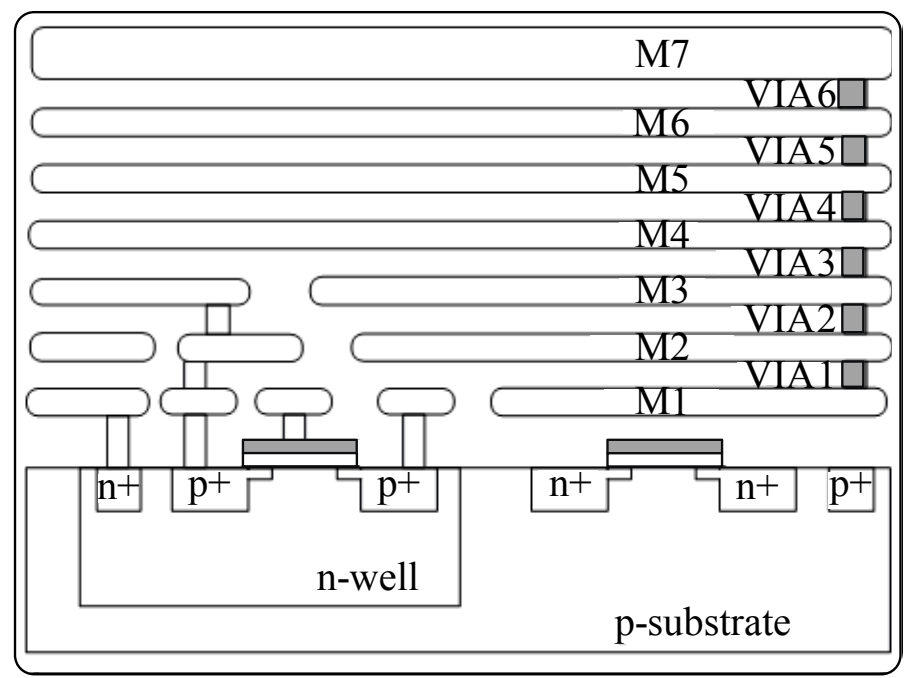

Figure 3.8: Standard CMOS metal layer arrangement.

In most CMOS manufacturing processes, the electrical connections between pixel components are routed through a series of metal layers that are between the imaging element of the system (lens) and the photodetector. It is preferred to have the opening between the light sensitive portion of the photodetector and the 
lens to be free of any metal interconnects to increase the quantum efficiency. In modern manufacturing processes, the metal lines within the pixel are comparable or smaller than the wavelength of visible light (400nm to $700 \mathrm{~nm})$. At these scales, the metal layers are not as opaque to visible light as bulk metal, and variations in the pattern control the probability that incident light at a particular wavelength will influence the photodetector.

With the scaling of CMOS technologies, the distance between metal layers also scales, opening up the possibility of using them in a grid structure for the absorption of electromagnetic waves.

\subsubsection{Transmittance efficiency and extinction ratio of wire- grid polarizers}

When randomly polarized light is transmitted through an ideal wire-grid polarizer, the electric fields orthogonal to the wires will be transmitted and electric fields parallel to the wire will be reflected, as seen in section 3.5. In practice however, wire-grid polarizers transmit some undesired parallel electric fields while reflecting some desired orthogonal electric field and also absorbing some of the incident light. Thus the transmitted electric field through a wiregrid polarizer can be modeled as having two components, an electric vector associated with the parallel electric field and an electric vector associated with the orthogonal electric field that are each scaled by a scalar value. The scalar value is proportional to the square root of the intensity associated with each polarization. Equation (3.24) is the resulting electric vector equation for unpolarized light passing through a wire-grid polarizer.

$$
\vec{E}=t(\hat{p} \cdot \vec{E}) \hat{p}+c(\hat{r} \cdot \vec{E}) \hat{r}
$$

where $E$ is the resultant electric field after transmission through the wire-grid polarizer and the scalar value for the parallel electric field is $t$ and the scalar value for the orthogonal electric field is $c$.

The irradiance of the plane polarized light passing through a polarizer shown in figure 3.9 is given by the "law of Malus" [3.25].

If the transmission axis of the polarization filter is parallel to the $x$-axis of the incident polarized light the transmitted intensity is given by equation (3.25).

$$
I=I_{0} \cos ^{2}(\varphi)
$$




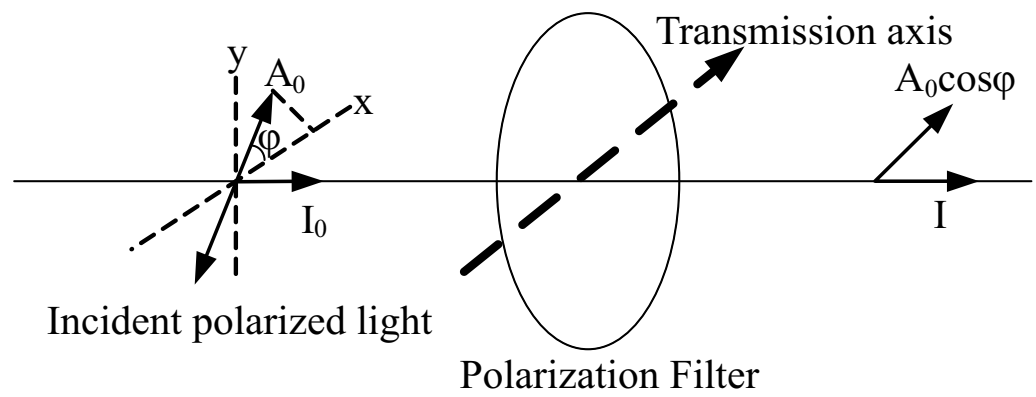

Figure 3.9: Intensity transmission through a polarizer, Malus law.

where $I$ is the transmitted intensity, $I_{0}$ is the maximum transmitted intensity and $\varphi$ is the angle between the polarizer's transmission axis and the plane of polarization of the incident light. Thus the maximum intensity that can be sensed by the detector is dependent of the angle of the transmission axis of the polarization filter and would result in a cosine wave with varying transmission axis angle.

Based on the transmission of intensity at a given transmission axis of a linear polarizer, linear polarizers are characterized by two main specifications: transmittance efficiency and extinction or contrast ratio.

The wire-grid polarizer's transmittance efficiency is the fraction of the total incident light that is transmitted through the linear polarizer. The maximum transmission in the linear polarizer is obtained when the polarizer orientation is aligned parallel to the orientation of the linear polarized component and the minimum is obtained when the polarizer orientation is aligned perpendicular to the linear polarized component of light.

The extinction ratio $(E R)$ is a measure of the polarization contrast of a linear polarizer and it is defined as the intensity ratio between the maximum and minimum throughputs of the polarizer when the wire-grid is aligned and crossed with respect to the light beam. The accuracy of the wire-grid polarizer's extinction ratio measurement depends on several factors such as the detector precision, analyzer alignment, stray light variations and light source stability. In an ideal testing environment with no light source or detector drift or background noise variations, the extinction ratio can be simply defined as

$$
E R=\frac{I_{p}}{I_{s}}
$$

where $I_{p}$ is the maximum transmittance parallel to the polarized beam and $I_{s}$ 
is the minimum transmittance perpendicular to the plane of the polarized beam. The contrast ratio for polarizers varies depending on the polarizer, ranging from around 10 to over $10^{7}$.

Extinction ratios can be further enhanced using two wire-grid polarizers in series with parallel transmission axis, where the overall extinction ratio is the product of the extinction ratios of the individual polarizers.

The transmittance of the transmagnetic (TM) polarization component is also important, since poor $T M$ transmittance would require a highly sensitive sensor element even with an excellent $E R$. In an ideal wire-grid polarizer $50 \%$ of the light intensity would be reflected in the s-polarization state and $50 \%$ transmitted in the $p$-polarization state. The polarizer would have a transmission efficiency of $100 \%$ and an infinite extinction ratio. Since unpolarized natural light consists of two orthogonal linear polarizations, polarization of light can only provide $50 \%$ of the light in the desired polarization state.

A novel wire grid polarization image sensor combining CCD imaging technology with nanotechnology has been recently been presented [3.26]. The aluminum nanowire optical filters are monolithically integrated directly on top of the imaging array as close as possible to the photosensitive element using a modified procedure of interference lithography. Fabricating these metallic nanowires with sub-wavelength dimensions has become feasible with the maturity of nanofabrication technologies and techniques [3.22]. The optical nanowire polarization filter made by first depositing a 70nm thin film of aluminum followed by $30 \mathrm{~nm}$ thin film deposition of $\mathrm{SiO}_{2}$ using $e$-beam evaporation. The maximum extinction ratio reported for an incident light wavelength of $625 \pm 5 \mathrm{~nm}$ and is 58 \pm 1.2 across the imaging array. The extinction ratios for green $(515 \pm 5 \mathrm{~nm})$ and blue $(460 \pm 5 \mathrm{~nm})$ wavelengths are $44 \pm 0.7$ and $30 \pm 0.5$ respectively. For incident light ( $625 \pm 5 \mathrm{~nm}$ wavelength) perpendicular to the surface of the imaging sensor, the extinction ratios are maximum and equal to 58 [3.26].

A high resolution liquid crystal micropolarimeter for visible linear $\left(0^{\circ}, 90^{\circ}\right)$ and circular polarization (right handed) imaging in real time has also been reported [3.27]. An extinction ratio as high as $\sim 1100$ at the wavelength of $500 \mathrm{~nm}$ is reported. The polarization filter is implemented by micro-patterning a liquid crystal layer on top of a $45^{\circ}$ oriented ultra-thin metal wire-grid polarizer. Large area aluminum nanowire grid with $40 \mathrm{~nm} / 78 \mathrm{~nm}$ space and $118 \mathrm{~nm}$ pitch, fabricated using full wafer immersion interference lithography is also available [3.22]. The wire grid is shown to perform as a highly efficient optical polarizer for deep ultraviolet down to $\sim 250 \mathrm{~nm}$. The average extinction ration at $300 \mathrm{~nm}$ is reported to be $\sim 400$. 
The metallic wire grid linear polarizers can also be made using the metal planes normally used for interconnections in modern CMOS processes with gatelengths of $180 \mathrm{~nm}$ or below. Because the size of these metal planes are smaller than the wavelength of visible light, they show a unique transmission behavior for optical waves. By proper design of the structures with gratings or holes in one or more metal layers, optical functionality such as polarization transmission can be achieved. A polarization-analyzing CMOS image sensor using $0.35 \mu \mathrm{m}$ 2-poly 4-metal standard CMOS technology was reported by [3.20], [3.21]. The sensor featured a monolithically embedded polarizer. The angle of the embedded polarizer was varied to realize a real time absolute measurement of the incident polarization angle. The extinction ratio of the embedded polarizer reported was as small as 2. Although the pixel-level performance was limited, the estimation schemes based on the variation of the polarizer angle provided promising performance for uniform illuminations.

Besides transmittance efficiency and extinction ratio, linear polarizers are also characterized using the degree of polarization $(D O P)$. The degree of polarization of a wire-grid polarizer in terms of maximum and minimum transmittance is given by

$$
E R=\frac{I_{p}-I_{s}}{I_{p}+I_{s}}
$$

where $I_{p}$ is the maximum transmittance parallel to the polarized beam and $I_{s}$ is the minimum transmittance perpendicular to the plane of the polarized beam.

Typically, for a totally polarized light a $D O P$ close to one is obtained while for a completely unpolarized light a $D O P$ of near zero is desired.

\subsection{Design of a polarization sensor}

In section 3.5 , its shown that to selectively absorb the electromagnetic waves in the visible spectrum the grid pitch should be very small. Thus a lower technology nodes, much lower than $0.18 \mu \mathrm{m}$, would be ideal to design the wire grid micropolarizers. However the CIS (CMOS Image sensor) process of UMC with 3-metal layers provide only $0.18 \mu \mathrm{m}$ photodiodes, the photodiodes for technology nodes below $0.18 \mu \mathrm{m}$ are not very well characterized. In a $0.18 \mu \mathrm{m}$ standard CMOS process the minimum allowable distance between two metals is $0.24 \mu \mathrm{m}$. A one-dimensional wire-grid polarizer implemented using the bottom metal layer (M1) is shown in the figure 3.10. 


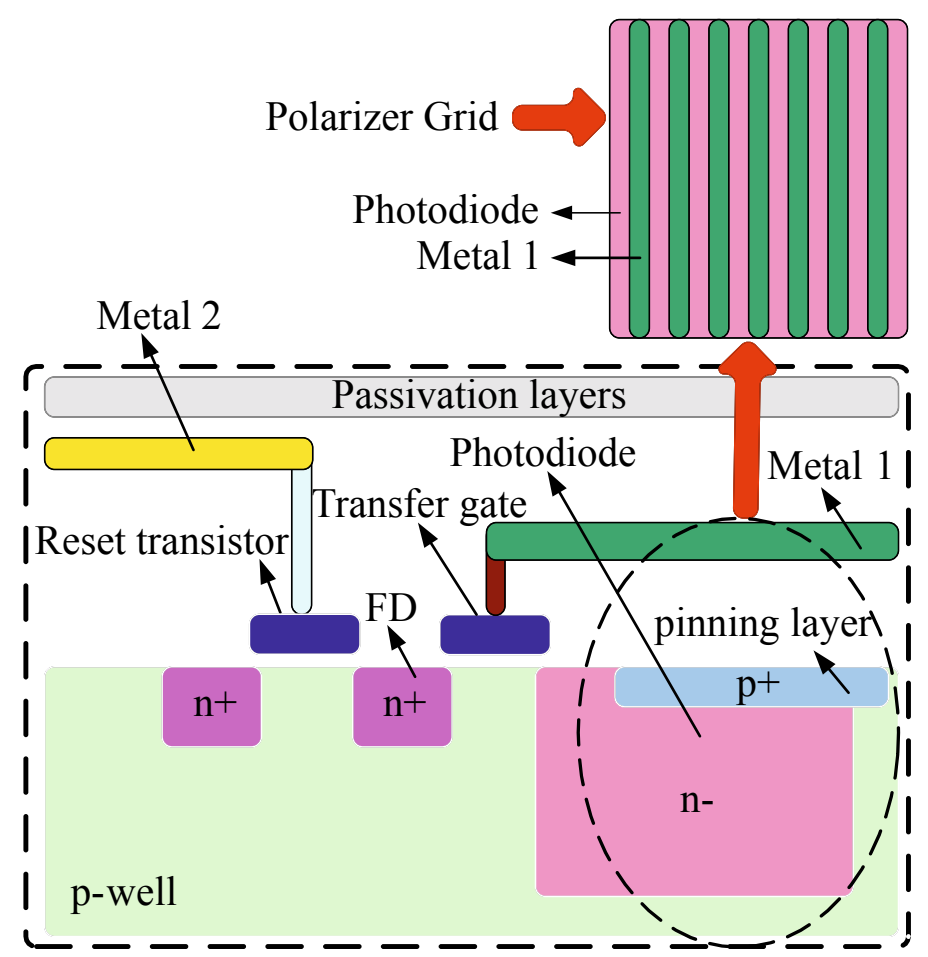

Figure 3.10: Pixel cross-section and top view of the implemented wire-grid polarizer.

The wire-grid polarizer is implemented over the pixel described in chapter 2. The thickness of the metal layer M1 used to create the wire-grid polarizer is $0.35 \mu \mathrm{m}$ while the width of metal wire is $0.24 \mu \mathrm{m}$. The choice of the thickness and the width of the metal wire are limited by the choice of the technology. The aspect ratio for the designed wire-grid micro-polarizer is 1.5:1. The duty cycle of the designed wire-grid micro-polarizer is $50 \%$ with a pitch of $0.48 \mu \mathrm{m}$. The wiregrid parameters are summarized in table 3.2. The grid metal thickness is fixed by the CMOS process metal thickness. Based on the selected grid width and height, the incident $p$-polarized light whose electric field is parallel to the grating is mostly transmitted and the s-polarized whose electric field is perpendicular to the grating is mostly transmitted and detected by the photodiode.

Figure 3.11(a) shows the implementation of the polarization analyzing CMOS image sensor operated in spatial mode. The wire-grid micro-polarizer was created with varying orientations over the pixel array to enable real time measurement of the polarization information. The active pixel array of 128 by 


\begin{tabular}{|l|l|}
\hline Metal thickness & $0.35 \mu \mathrm{m}$ \\
\hline Metal width & $0.24 \mu \mathrm{m}$ \\
\hline Aspect ratio & $1.5: 1$ \\
\hline Grid pitch & $0.48 \mu \mathrm{m}$ \\
\hline Duty cycle & $50 \%$ \\
\hline
\end{tabular}

Table 3.2: Wire-grid parameters.

128 pixels was split into two regions of $64 \times 128$ pixels. One region of $64 \times 128$ pixels was used for normal imaging applications as described in chapter 2 with the photodiode free of wire-grid micro-polarizer while the other region was made polarization sensitive by using the wire-grid micro-polarizer on top of the photodiode.

The polarization analyzing region was further split into two regions of $64 \times 64$ arrays, referred to as sense region 1 and 2 respectively. A $2 \times 2$ pixel array in the sense region 1 consists of two pixels (A and $\mathrm{B}$ ) measuring the intensity, while the other two measure the $0^{\circ}(\mathrm{D})$, and $90^{\circ}(\mathrm{C})$ polarized intensity respectively. A $2 \times 2$ pixel array in the sense region 2 has one pixel recording the intensity of the light (A) while the other three record the $0^{\circ}$ (B), $45^{\circ}$ (C) and $90^{\circ}$ (D) polarized intensity.

Figure 3.11(b) shows the transmission image showing the attenuation of the light in the regions where the photodiode has integrated metallic wire-grid polarizer. The sense region 2 has three pixels with wire-grid polarizer thus the attenuation of light is greater compared to sense region 1.

\subsection{Polarization measurements}

A partially linearly polarized light has three descriptors: intensity, degree of polarization and $e$-vector angle. These descriptors are measured using the variations in the pixel intensity with the changes in the transmission axis of the linear polarizers. The transmission efficiency is a measure of the partially linearly polarized light intensity that has been transmitted through the linear polarizer. The transmission efficiency, extinction ratio and degree of polarization of the designed wire-grid micro-polarizer are measured and are found to be fully functional as a linear polarizer. 


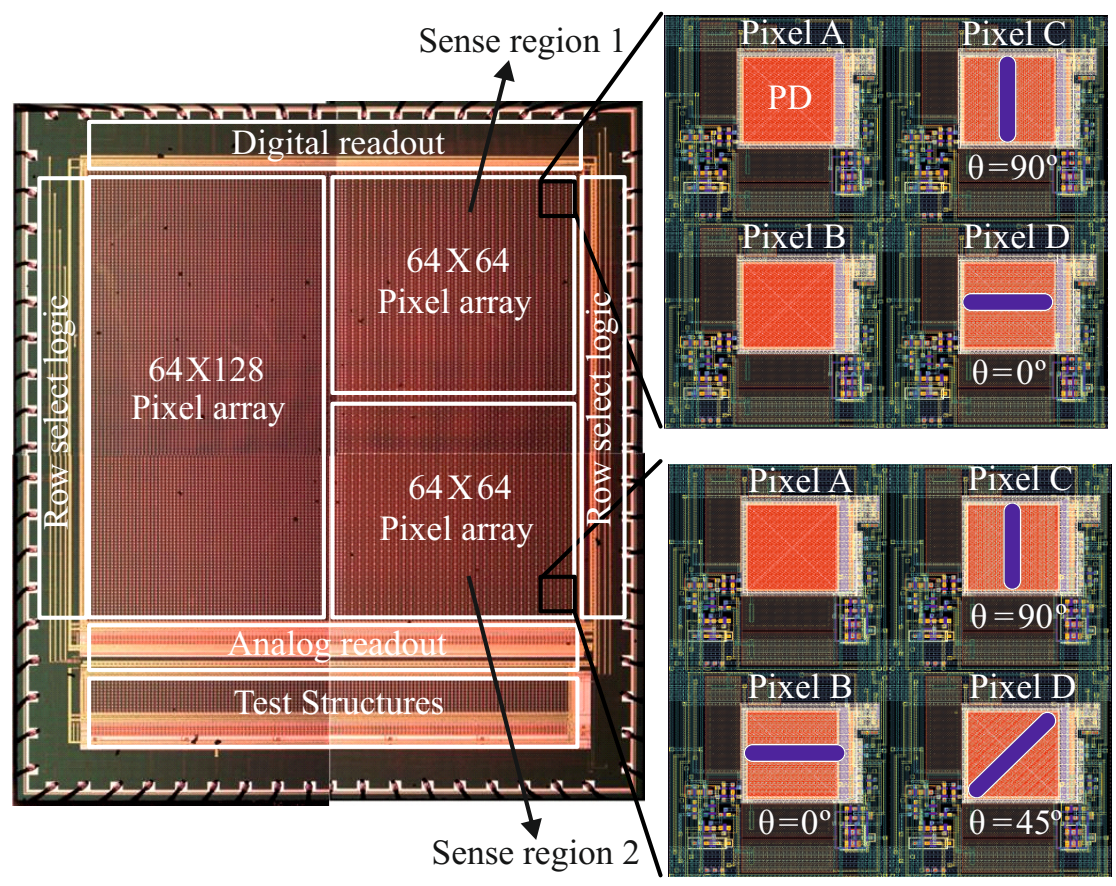

(a)

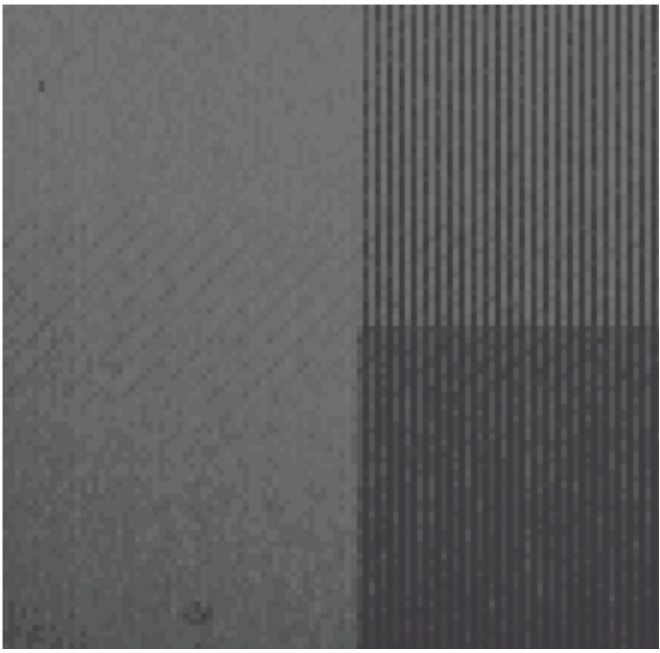

(b)

Figure 3.11: (a) Sensor regions with different polarizing angles, (b) transmission photograph of the wire-grid polarizer. 


\subsubsection{Measurement setup}

The measurement setup for measuring the transmittance and the extinction ratio is shown in figure 3.12. In comparison to the measurement setup shown in the figure 2.34, here an additional linear external polarizer is used. The sensor was illuminated with a polarized light obtained by passing the light from a DC light source through a linear polarizer. The DC light source used is an Philips halogen $12 \mathrm{~V}, 20 \mathrm{~W}$ bulb, set to radiate $400 \mu \mathrm{W} / \mathrm{cm}^{2}$. The optical power was measured using the optical power meter TQ8210. The transmission axis of the linear polarizer was then varied from $0^{\circ}$ to $180^{\circ}$ in steps of $15^{\circ}$ to vary the polarization angle of the light reaching the image sensor.

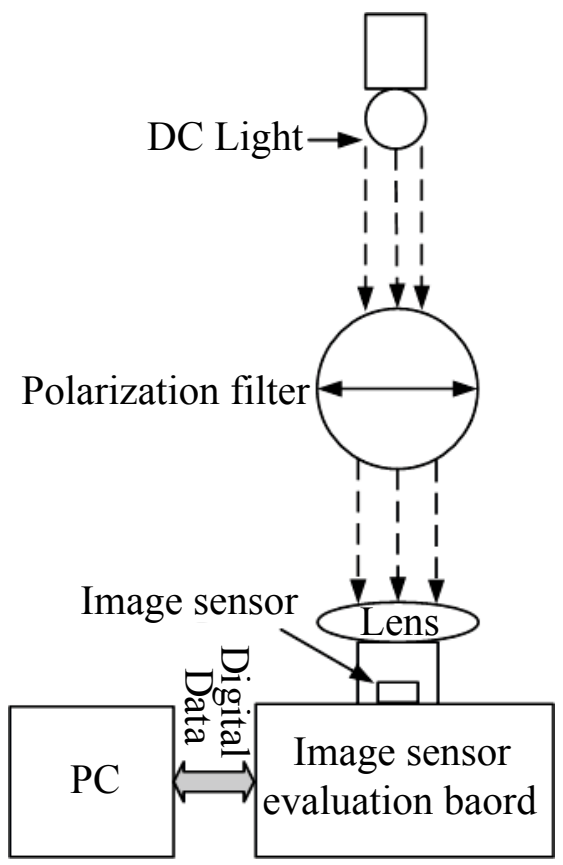

Figure 3.12: Wire-grid measurement setup.

The corresponding analog output of the pixels sensitive to $0^{\circ}$ and $90^{\circ}$ in the polarization sense region 1 and $0^{\circ}, 45^{\circ}$ and $90^{\circ}$ in the polarization sense region 2 (see section 3.6) are recorded. The analog signal from the image sensor is digitized using an external $A D C$ and then analyzed using a PC. The obtained output is normalized with respect to the intensity obtained at the intensity sensitive pixel, to compensate for any variation in the light intensity and pixel sensitivity over the entire imaging array. The normalized output is the 
transmittance of the wire-grid polarizer.

An average value of the intensities of the pixels sensitive to $0^{\circ}$ and $90^{\circ}$ in the sense region 1 and the pixels sensitive to $0^{\circ}, 45^{\circ}$ and $90^{\circ}$ in the sense region 2 is computed for 30 frames using the following equation:

$$
p_{\text {avg }(\text { with linear polarizer })}(x, y)=\frac{1}{N} \sum_{n=1}^{N} p_{n}(x, y)
$$

where $p_{n}(x, y)$ is the measured pixel intensity in frame $n, x$ and $y$ correspond to the row and column address of the pixel in the sensor array and $N$ is the number of frames selected. At the beginning of the experiment the mean of an array of $20 \times 20$ pixels without the linear polarizer is calculated as in equation (3.29). The result is used as a normalization factor.

$$
p_{\text {avg }(\text { without linear polarizer })}(x, y)=\frac{1}{X Y N} \sum_{n=1}^{N} \sum_{X=0 Y=0}^{X-1 Y-1} \sum_{n} p_{n}(x, y)
$$

where $X$ and $Y$ are the pixel array dimensions and $N$ is the total number of frames used to compute the mean. The normalized intensity is then obtained by dividing the mean pixel intensity with the linear polarizer (equation (3.28)) by the mean pixel intensity without the linear polarizer (equation (3.29)), as shown in equation (3.30)).

$$
\text { Normalized } p_{\text {avg }}(x, y)=\frac{\frac{1}{N} \sum_{n=1}^{N} p_{n}(x, y)}{\frac{1}{X Y N} \sum_{n=1}^{N} \sum_{X=0}^{X-1 Y-1} \sum_{X=0} p_{n}(x, y)}
$$

\subsubsection{Analog polarization measurements}

The polarization transmission path of the incident light ray in the figure 3.12 can be simplified as shown in the figure 3.13. The polarizer is the external polarization filter and the analyzer is the metallic wire-grid polarization filter patterned on top of the photodiodes of the designed polarization image sensor. When an unpolarized light wave with intensity $I_{0}$ is incident on the polarizer, the intensity of the transmitted light wave is $I_{1}$. When this light wave is further incident on the analyzer the intensity $I_{2}$ is obtained after transmission, which is 
then detected by the detector.

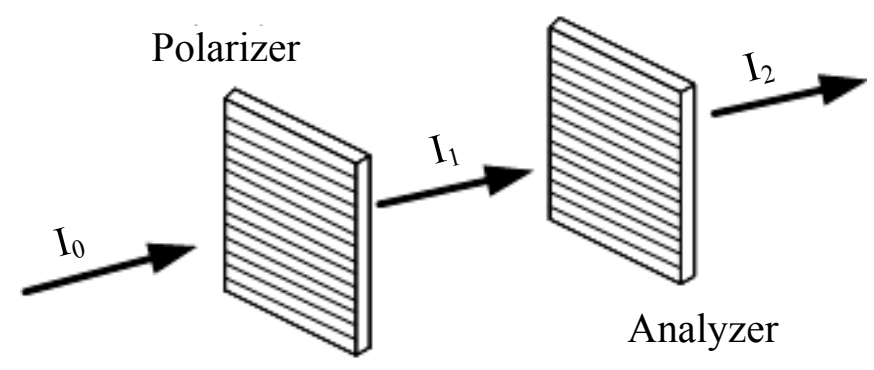

Figure 3.13: Polarizer and analyzer arrangement.

In the polarization sense region 1 of the image sensor (figure 3.11a), to measure the $90^{\circ}$ polarization behavior, the transmission axis of the polarizer was varied from $0^{\circ}$ to $180^{\circ}$ and the corresponding analog output of the pixel with a $90^{\circ}$ transmission axis analyzer was measured. For the $0^{\circ}$ polarization behavior, the transmission axis of the polarizer was varied from $0^{\circ}$ to $180^{\circ}$ and the corresponding analog output of the pixel with a $0^{\circ}$ transmission axis analyzer was measured. The normalized transmittance as a function of the transmission axis of the linear polarizer (incident polarization angle) for the sense region 1 is shown in figure 3.14. The transmitted radiance of a linearly polarized light through the linear polarizer at a pixel array in an image is seen to vary sinusoidally as function of the polarizer transmission axis.

The maximum $I_{\max }$ of the sinusoid occurs when the polarizer orientation is parallel to the orientation of the linear polarized component and the minimum $I_{\min }$ occurs when the polarizer orientation is perpendicular to the linear polarized component. In the sense region 1 , for $0^{\circ}$ polarization the maximum transmittance achieved is $38.9 \%$ and the minimum transmittance observed is $7 \%$. For $90^{\circ}$ polarization the maximum transmittance obtained is $44.4 \%$ and the minimum transmittance is $0.1 \%$.

The experiment was then repeated in the polarization sense region 2 of the image sensor (figure 3.11a). The normalized transmittance as a function of the transmission axis of the linear polarizer (incident polarization angle) for the sense region 2 is shown in figure 3.15. For the polarization sense region 2 , for $0^{\circ}$ polarization the maximum transmittance obtained was $38.4 \%$ while the minimum transmittance was $5.45 \%$. For $90^{\circ}$ polarization the maximum transmittance observed was $42.4 \%$ while the minimum transmittance observed is $0.6 \%$.

The extinction ratio can be calculated using equation (3.26). The calculated 


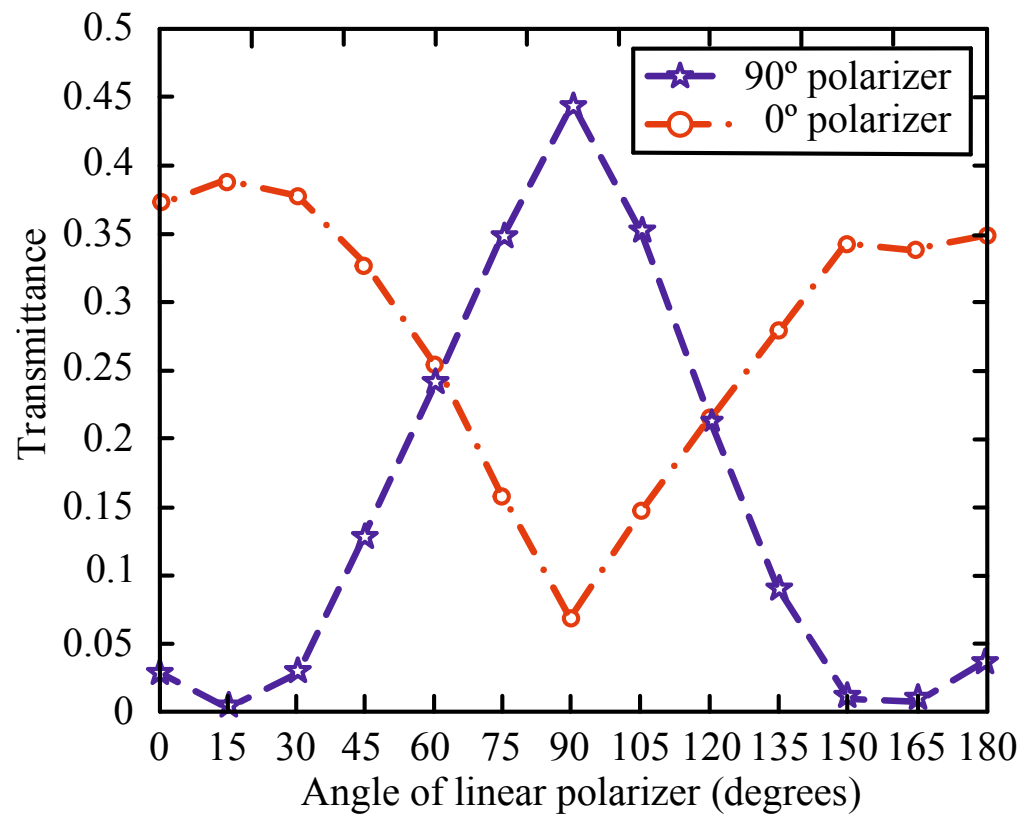

Figure 3.14: Polarization sense region 1: $90^{\circ}$ and $0^{\circ}$ polarization transmittance.

extinction ratio for the linear polarizer in the sense region 1 is 6.3 and in the sense region 2 is 7.7 [3.28] at $\lambda=550 \mathrm{~nm}$. The obtained extinction ratios are higher than reported in literature for a similar polarization detection CMOS image sensor, where an extinction ratio of 2.3 is presented in [3.20], [3.21] with a $0.95 \mu \mathrm{m}$ wire grid pitch designed in a $0.35 \mu \mathrm{m}$ 2-poly 4-metal standard CMOS at $\lambda=589 \mathrm{~nm}$ and 6 at $\lambda=650 \mathrm{~nm}$ in [3.29] using a photo-patterned micropolarizer array.

The absorption of the $E M$ waves to completely $s$-polarize or $p$-polarize the transmitted wave through the wire-grid is dependent on the pitch of the wiregrid and so the extinction ratio also varies with the pitch. In our case, a higher extinction ratio is obtained than previously reported in literature because of the lower wire-grid pitch used in the image sensor. The mean maximum $\left(T_{\max }\right)$ and the minimum transmittance $\left(T_{\min }\right)$ for $0^{\circ}$ and $90^{\circ}$ polarization sensitive pixels in the polarization region 1 and 2 are shown in the table 3.3.

\begin{tabular}{|c|c|c|c|c|c|}
\hline Region & $T_{\max }\left(0^{\circ}\right)$ & $T_{\min }\left(0^{\circ}\right)$ & $T_{\max }\left(90^{\circ}\right)$ & $T_{\min }\left(90^{\circ}\right)$ & $E R$ \\
\hline 1 & 0.389 & 0.07 & 0.444 & 0.01 & 6.3 \\
\hline 2 & 0.384 & 0.0545 & 0.424 & 0.06 & 7.7 \\
\hline
\end{tabular}

Table 3.3: Transmittance (\%) and extinction ratio (ER). 


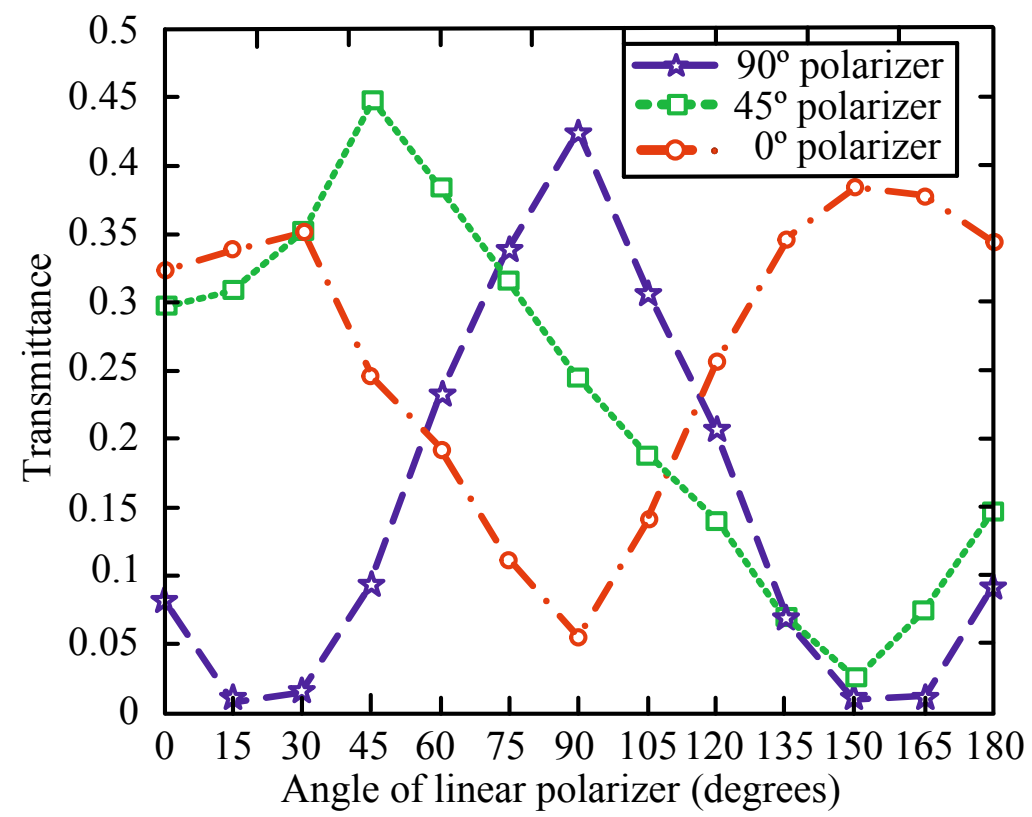

Figure 3.15: Polarization sense region $2: 90^{\circ}, 45^{\circ}$ and $0^{\circ}$ polarization transmittances.

The sense region 2 has an additional linear polarizer oriented at $45^{\circ}$. The maximum and the minimum transmittance efficiency for the $45^{\circ}$ sensitive pixel are $44.6 \%$ and $2 \%$. The extinction ratio is thus calculated to be 22 .

A performance comparison of the designed polarization sensor with the available wire grid polarizers in literature is shown in table 3.4

The degree of polarization variation for the sense region 1 and 2 is shown in the figure 3.16. For a perfect polarizer $I_{s}$ in equation (3.27) is equal to 1 , which means full transmission of polarized light whose electric field is perpendicular to the wire-grid and $I_{p}$ is equal to 0 , meaning complete blockage of the polarized light whose electric field is parallel to the wire-grid.

The degree of polarization is shown to vary between 1 and -0.8 . Ideally it is expected to vary between 1 and -1 . The maximum value of the $D O P$ is obtained at $0^{\circ}$ and $180^{\circ}$ while the minimum is obtained at $90^{\circ}$ angle of the linear polarizer. 
3.8. Wavelength selection using metal grid

\begin{tabular}{|l|l|l|l|l|}
\hline & {$[3.26]$} & {$[3.27]$} & {$[3.21]$} & This work \\
\hline Imager type & CCD & CMOS & CMOS & CMOS \\
\hline Polarizer type & $\begin{array}{l}\text { Aluminum } \\
\text { nanowires }\end{array}$ & $\begin{array}{l}\text { Micro- } \\
\text { patterning } \\
\text { liquid crystal } \\
\text { metal wire- } \\
\text { polarizer }\end{array}$ & $\begin{array}{l}\text { Standard } \\
\text { CMOS } \\
\text { metal } \\
\text { layers }\end{array}$ & $\begin{array}{l}\text { Standard } \\
\text { CMOS } \\
\text { metal } \\
\text { layers }\end{array}$ \\
\hline $\begin{array}{l}\text { Spatial } \\
\text { orientations }\end{array}$ & $\begin{array}{l}0^{\circ}, 45^{\circ}, \\
90^{\circ} \& 135^{\circ}\end{array}$ & $\begin{array}{l}0^{\circ}, 90^{\circ} \& \\
\text { right-handed } \\
\text { circular } \\
\text { polarized }\end{array}$ & $0^{\circ} \& 90^{\circ}$ & $\begin{array}{l}0^{\circ}, 45^{\circ} \\
\& 90^{\circ}\end{array}$ \\
\hline Pixel pitch & $7.4 \mu \mathrm{m}$ & $5 \mu \mathrm{m}$ & $20 \mu \mathrm{m}$ & $25 \mu \mathrm{m}$ \\
\hline Grating thickness & $0.14 \mu \mathrm{m}$ & $5 \mu \mathrm{m}$ & - & $0.35 \mu \mathrm{m}$ \\
\hline Grating width & $0.75 \mu \mathrm{m}$ & - & $0.6 \mu \mathrm{m}$ & $0.24 \mu \mathrm{m}$ \\
\hline Grating pitch & $0.14 \mu \mathrm{m}$ & - & $1.2 \mu \mathrm{m}$ & $0.48 \mu \mathrm{m}$ \\
\hline Extinction ratio & $\sim 60$ & $\begin{array}{l}\sim 1100 \\
(\lambda=500 \mathrm{~nm})\end{array}$ & $\begin{array}{l}2.3 \\
(\lambda=633 \mathrm{~nm})\end{array}$ & $\begin{array}{l}6.6 \& 7.7 \\
(\lambda=550 \mathrm{~nm})\end{array}$ \\
\hline
\end{tabular}

Table 3.4: Performance comparison of the available polarization sensors.

\subsection{Wavelength selection using metal grid}

The extinction ratio of a wire-grid polarizer depends on the incident wavelength and oblique angle incidence of the incident electromagnetic wave. The transmission decreases rapidly for the $s$-polarization with increasing wavelength or decreasing width of the opening between the metal wire-grid [3.30]. In contrast, the transmission of the $p$-polarized wave remains high for long wavelengths. The $E R$ is found to be wavelength dependent: a low $E R$ value is obtained for shorter wavelengths [3.30]. Transmission for $p$-polarization is at least two orders of magnitude higher than $s$-polarization in the $600-900 \mathrm{~nm}$ range. To control the incident wavelength, regular metal grids have been used for a long time as filters, transmitting some wavelength and blocking others. The selective transmission of the wavelength can further be used as a color filter.

A novel technique for color detection using metal gratings on top of the photodetectors in standard CMOS technology was first introduced in 2001 [3.31]. The metal gratings are two-dimensional structures with different periodicity. Metal gratings have not been used for color filtering until recent years because the grid spacing must be very small for visible wavelengths; this has been difficult until $0.18 \mu \mathrm{m}$ CMOS technology became available [3.32]. The metal layers are 


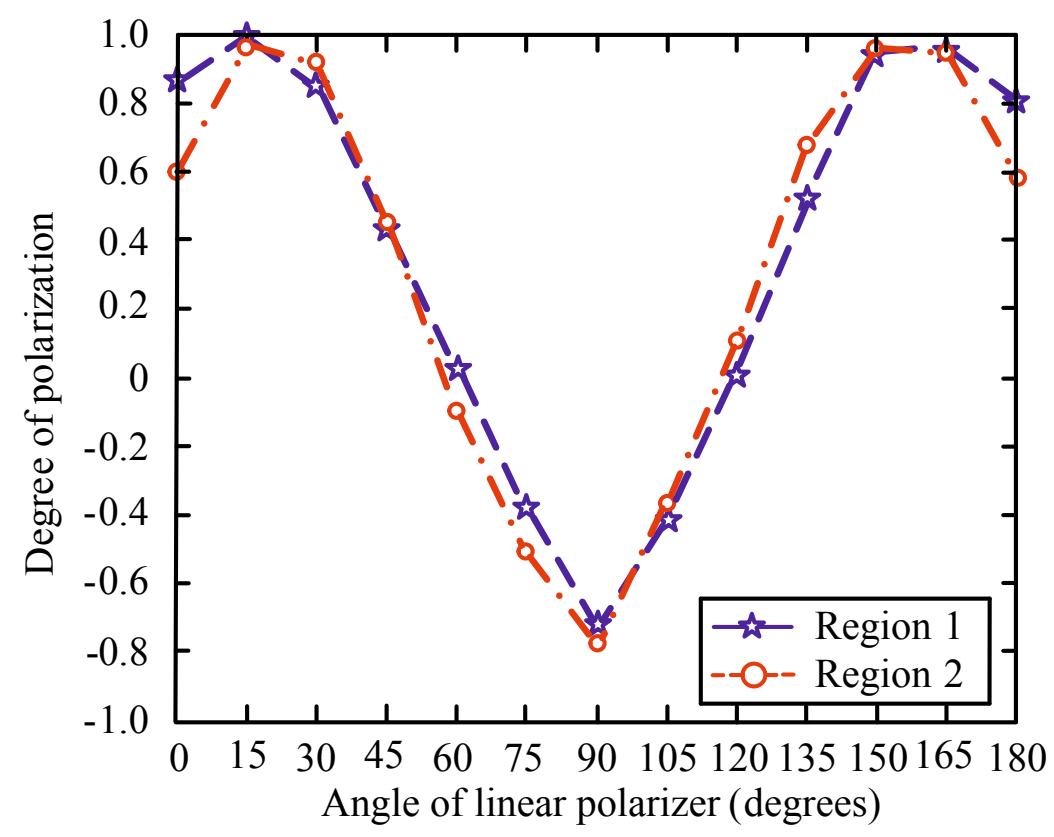

Figure 3.16: Degree of polarization of the wire-grid.

deposited on top of the photodetectors, and since commercial IC fabrication processes have between two and eight layers of metal available, integrating metal grid light filters with the detectors eliminates the need for additional color filter array manufacturing steps. The short distance between the light filter and the photodetector has the advantage of reducing color crosstalk and pixel vignetting [3.31]. The light efficiency decreases due to the loss of light in the path from a light source to the pixel in the presence of wire-grid polarizer's. However for applications like for example $L C D s$ which contains two layers of polarizer's and a color sheet the light efficiency is very low, near $10 \%$. The patterned metal gratings on the pixel will help to detect both the polarization and the color allowing for increased light efficiency compared to conventional LCDs type applications and also allow for multispectral imaging.

A simulation was performed to check the feasibility of selective transmittance of a desired wavelength (color filter) using one-dimension or two-dimensional metal patterns on top of the photodetector in a CMOS or CCD image sensor pixel. A dual layer aluminum grid with a width of $0.2 \mu \mathrm{m}$ and a spacing of $0.1 \mu \mathrm{m}$ is used as a basic structure to design a color filter as shown in figure 3.17.

A two-dimensional forward difference time domain (FDTD) method of solving 


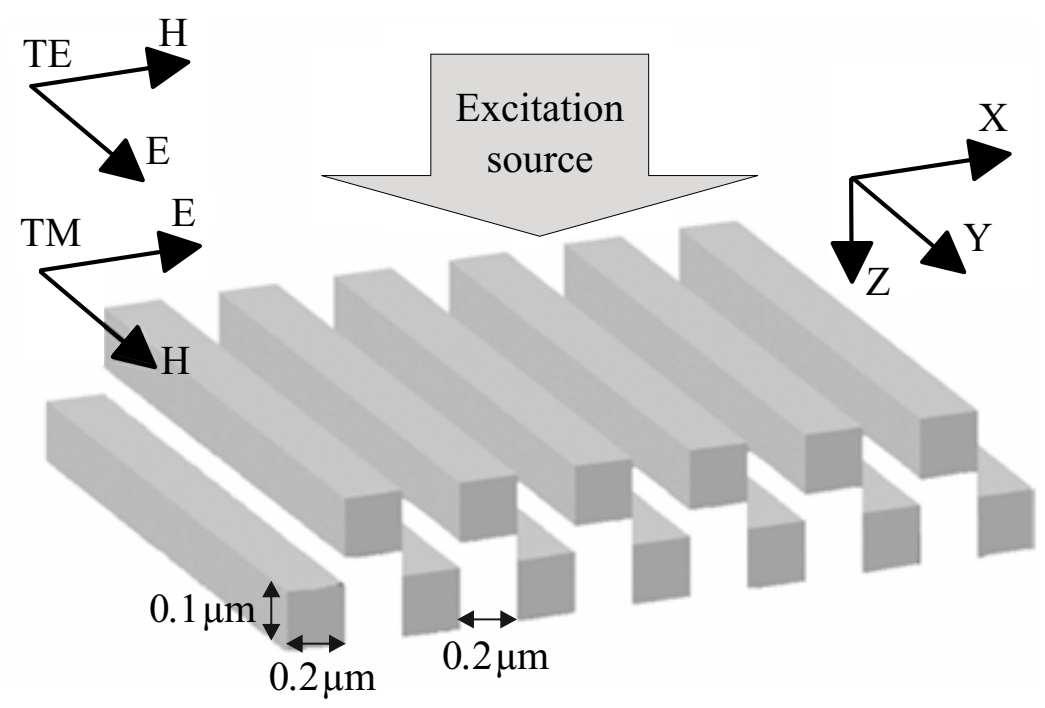

Figure 3.17: 2-layer wire-grid polarizer.

the Maxwell's equations for the incident electromagnetic wave field is used to simplify the simulations. FDTD is a time-domain approach to numerically solve the Maxwell's equations by continuously solving the electric and magnetic fields of the incident electromagnetic field over time to simulate the propagation of the waves.

A broadband Gaussian-modulated pulsed light source expressed by equation (3.31) is used to illuminate the wire-grid filter along the positive $z$ direction.

$$
T(t)=\exp \left[-\frac{1}{2}\left(\frac{t-t_{\text {off }}}{t_{w}}\right)\right] \sin (\omega t)
$$

where $t_{\text {off }}$ is the offset time, $t_{w}$ the half width of the pulse, and $\omega$ the central frequency of the source. Using this broadband excitation source, information of all the frequencies could be obtained in a single calculation. Due to the invariance of the structure along the $y$-axis, the transverse-magnetic (TM) mode, consisting of the field components $E_{x}, H_{y}$ and $E_{z}$ and the transverse-electric (TE) mode, consisting of $E_{y}, H_{y}$ and $E_{z}$ are completely decoupled.

Figure 3.18 shows the transmittance and reflection of an excitation signal polarized in $0^{\circ}$ and $90^{\circ}$, applied in the perpendicular $(z)$ direction to the metal grid structure with $0^{\circ}$ phase shift between the two metal layers (with the edge of the top and bottom metal layer coinciding). Figure 3.18(a) shows that for a $0^{\circ}$ 
polarized incident signal, the wire-grid structure transmits all the three primary colors. However for $90^{\circ}$ polarized incident signal, figure 3.18(b), the wire-grid filter shows a reduced transmission for wavelengths of 530nm and above, thus effectively working as a blue filter with transmission maximum between $400 \mathrm{~nm}$ and 500nm.

The transmittance and reflection of the $0^{\circ}$ and $90^{\circ}$ polarized excitation signals through the metallic wire-grid when the top metal layer in figure 3.17 is shifted by $0.1 \mu \mathrm{m}$ in the $x$ direction to have a $90^{\circ}$ phase shift between the two metal layers (an edge distance of $0.1 \mu \mathrm{m}$ between the top and the bottom metal layers) are shown in the figure 3.19. In this arrangement, for the $0^{\circ}$ polarized incident signal the maximum transmission peak occurs between $590 \mathrm{~nm}$ to $620 \mathrm{~nm}$ thus transmitting the red color of the visible spectrum while attenuating the green and the blue light. For $90^{\circ}$ polarized incident signal the wavelengths above $530 \mathrm{~nm}$ are completely blocked thus effectively blocking the red and the green color of the visible spectrum while transmitting the blue color.

The transmittance and reflection characteristics obtained for $0^{\circ}$ and $90^{\circ}$ polarized excitation signal through the metallic wire-grid when the top metal layer in figure 3.17 is further shifted by $0.1 \mu \mathrm{m}$ to obtain an $180^{\circ}$ phase shift between the two metal layers (edge distance of $0.2 \mu \mathrm{m}$ between the top and the bottom metal layers) are shown in the figure 3.20. In this configuration both for the $0^{\circ}$ and $90^{\circ}$ polarized incident signal only the blue region of the visible spectrum is transmitted while the red and the green are effectively attenuated or blocked.

The results demonstrate transmittance and responsivities potentially suitable for color filtering. By controlling the wavelength responsivity in the pixel design process, the polarization information for the responsive wavelength can also be fixed. There is a loss of transmission efficiency due to the increased attenuation and thus this will not be suited for high resolution imaging but it would help in polarization imaging.

Besides depending on the wavelength of the incident light, the $E R$ is also a function of the angle of incidence of the light ray. At a specular interface between a dielectric and absorbing material such as a metal or semiconductor, the angle of reflection of an incident light is equal but opposite to the angle of incidence, while the transmitted ray is refracted in accordance with Snell's law. The reflectance and transmittance are a function of the angle of incidence and the real and complex refractive indices of the materials. They are also functions of the polarization of the incident ray in accordance with the Fresnel's formulae. At the Brewster's angle, the reflected light is completely polarized perpendicular to 
the plane of incidence. Brewster's angle depends on the indices of refraction of the incident medium $\left(n_{1}\right)$ and the reflecting medium $\left(n_{2}\right)$. A detailed review of the Fresnel's equations and Brewster's angle is provided in chapter 4.

The polarization effects increase with increasing angle of incidence of the incident light [3.33]. At angles greater than $0^{\circ}$, the component of lightwaves vibrating parallel to the plane of incidence and reflection ( $p$-plane) will be filtered differently than the component vibrating perpendicular to the plane of incidence ( $s$-plane). The reflectance of the $p$-polarization decreases with increasing angle of incidence to a minimum, at the quasi polarizing angle, and then increases towards one as the polarizing angle approaches $90^{\circ}$. The reflectance of the $s$-polarization monotonically increases towards one as the polarizing angle approaches $90^{\circ}$. The extinction ratio is shown to rapidly decrease for higher incident angles, for very high incident angles the polarization information is completely lost due to the pixel crosstalk [3.26].

Furthermore both polarization components also undergo a phase shift on reflection upon the angle of incidence. The $s$-polarization phase shift increases monotonically towards $\pi$ radians and that of the $p$-polarization decreases monotonically towards zero as the polarizing angle approaches $90^{\circ}$. The change in the polarization behavior with changes in the angle of incidence affects the extinction ratios of the linear polarizers.

A wide field of view camera with a high angular resolution would be able to capture the polarization information over a wide field of view and this would naturally increase the sensitivity of the polarization or $E R$ measurements. 


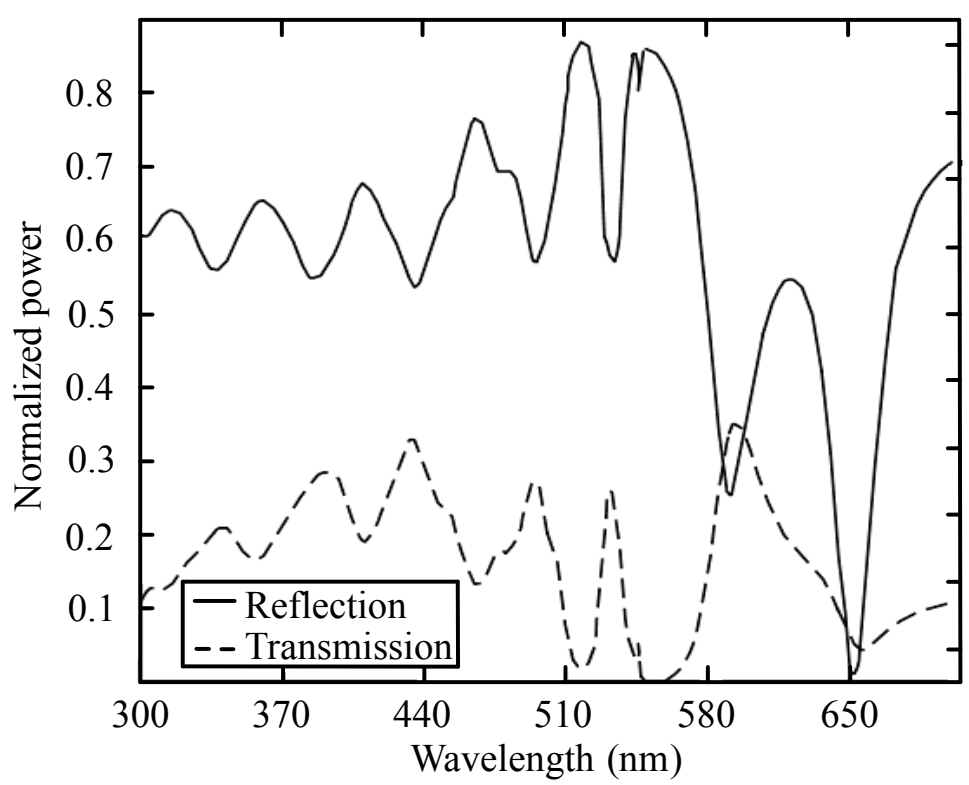

(a)

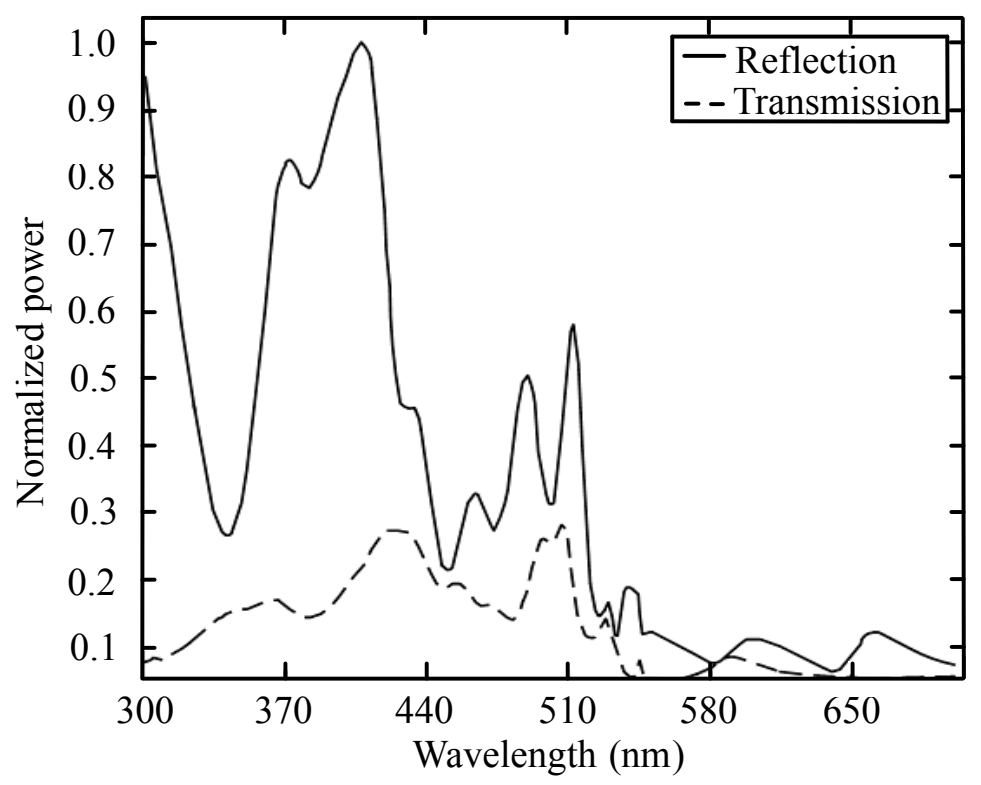

(b)

Figure 3.18: $0^{\circ}$ phase shift between the metal layers, reflection and transmission of (a) $0^{\circ}$ polarized signal $\left.b\right) 90^{\circ}$ polarized signal. 


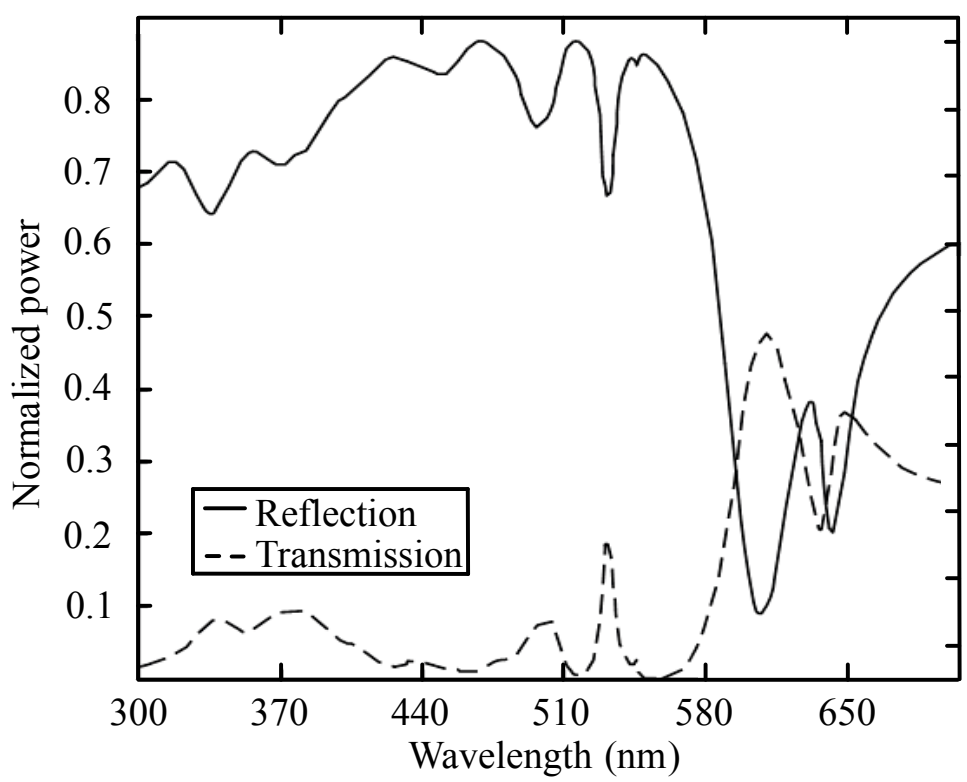

(a)

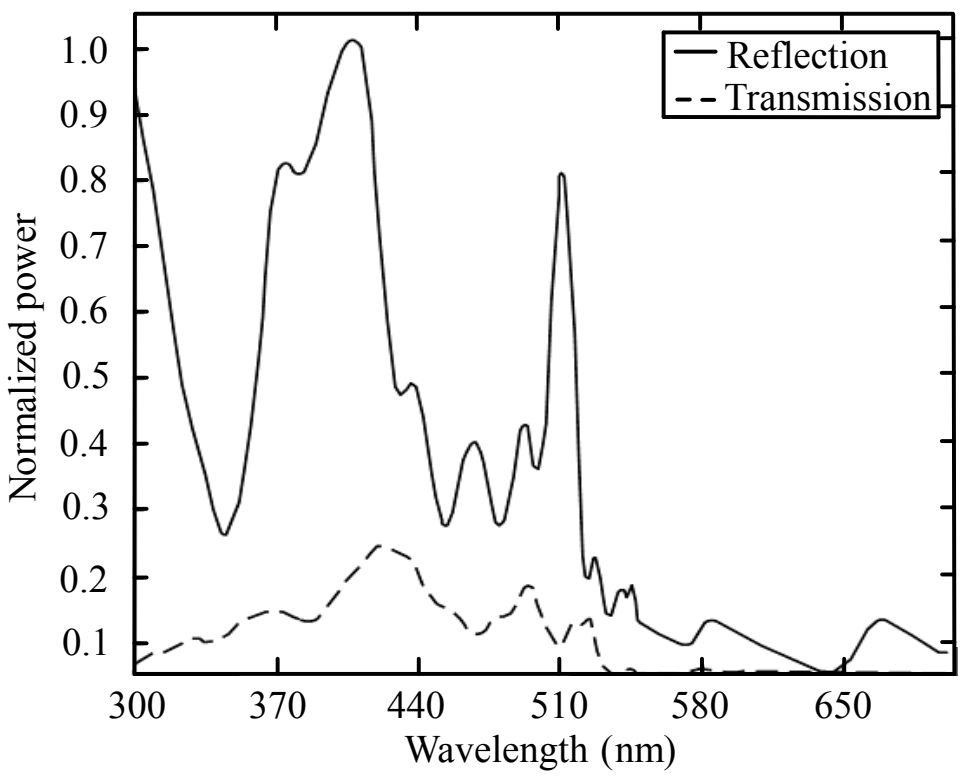

(b)

Figure 3.19: $90^{\circ}$ phase shift between the metal layers, reflection and transmission of (a) $0^{\circ}$ polarized signal b) $90^{\circ}$ polarized signal. 


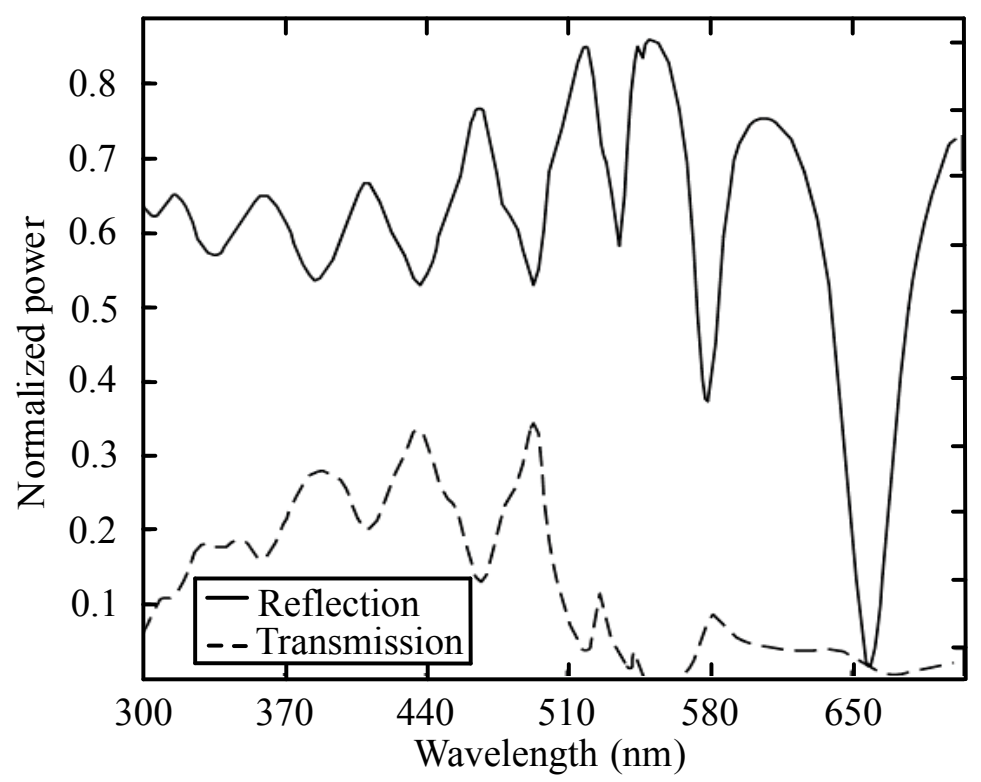

(a)

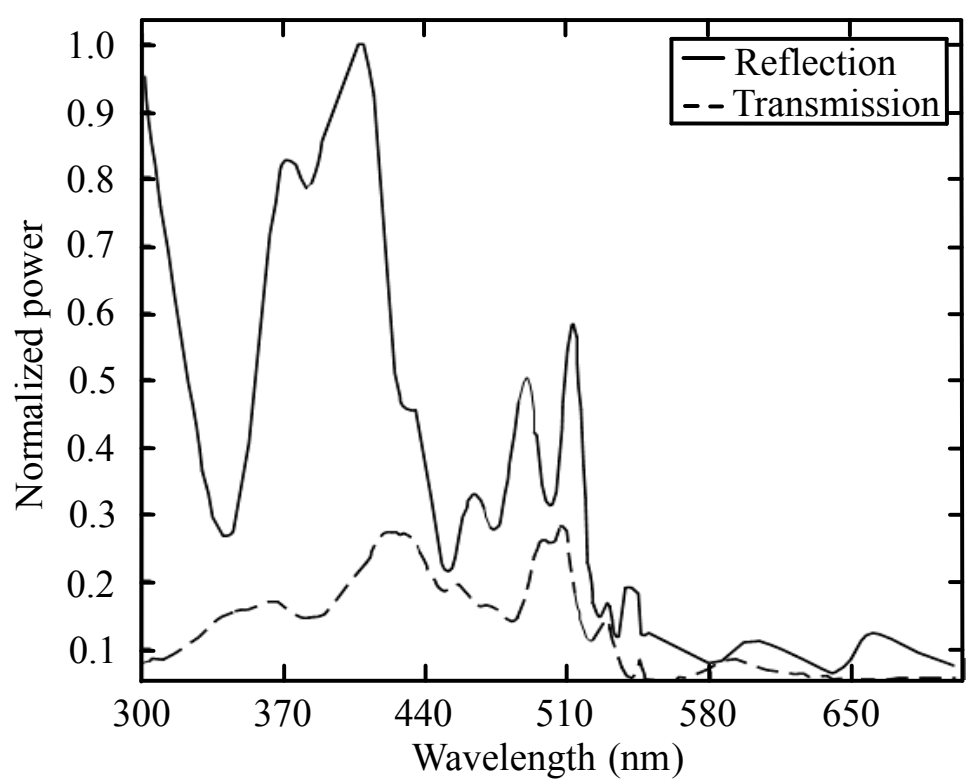

(b)

Figure 3.20: $180^{\circ}$ phase shift between the metal layers, reflection and transmission of $(a) 0^{\circ}$ polarized signal b) $90^{\circ}$ polarized signal. 


\subsection{Conclusions}

A CMOS image sensor using a metallic wire-grid micropolarizer using the standard CMOS metal layers was designed and is shown to be fully functional. The different orientation angles of the wire-grid senses different linear polarization of the light. Extinction ratios of 6.3 and 7.7 were achieved in the two sense regions of the sensor. The absorption of the $E M$ waves to completely $s$ polarize or $p$-polarize the transmitted wave through the wire-grid is dependent on the pitch of the wire-grid and so the extinction ratio also varies with the pitch.

\subsection{References}

[3.1] G. Bernard and R. Wehner, "Functional similarities between polarization vision and color vision," Vision Research, vol. 17, pp. 1019-1028, 1977.

[3.2] R. Wehner, "Polarization vision-a uniform sensory capacity?," Journal of Experimental Biology, vol. 204, pp. 2589-2596, 2001.

[3.3] D. Reiley, Polarization in optical design. PhD thesis, University of Alabama, 1993.

[3.4] G. Horva'th and D. Varju', Polarized light in animal vision. Berlin, Heidelberg, New York: Springer, 2003.

[3.5] http://www.microscopyu.com/articles/polarized/index.html.

[3.6] G. Stokes, "On the comppostion and resolution of streams of polarized light from different sources," Transactions of Cambridge Philosophical Society, vol. 9, pp. 399-416, 1852. Reprinted in Mathematics and Physics Papers, vol. 3, Cambridge University press, pp. 233, 1901.

[3.7] H. Poincaré, Theorie Mathematique de la Lumiere, vol. 2, ch. 12. Gauthiers-Villars, 1892.

[3.8] H. Jerrard, "Transmission of light through birefringent and optically active media: the poincaré sphere," Journal of Optical Society of America, vol. 44, no. 8, pp. 634-640, 1954.

[3.9] D. Nilsson, T. Labhart, and E. Meyer, "Photoreceptor design and optical properties affecting polarization sensitivity in ants and crickets," Journal of Comparitive Physiology A-Sensory Neural and Behavioural Physiology, vol. 161, pp. 645-658, 1987. 
[3.10] T. Lambart and E. Meyer, "Neural mechanism in insect navigation: polarization compass and odometer," Current Opinion in Neurobiology, vol. 12, no. 6, pp. 707-714, 2002.

[3.11] J. Tyo, D. Goldstein, D. Chenault, and J. Shaw, "A review of passive imaging polarimetery for remote sensing applications," Applied Optics, vol. 45, pp. 5453-5469, 2006.

[3.12] A. Andreou and Z. Kalayjian, "Polarization imaging: principles and integrated polarimeters," IEEE Sensors Journal, vol. 2, no. 6, pp. 566-576, 2002.

[3.13] X. Zhao, F. Boussaid, A. Bermak, and V. Chigrinov, "Thin photopatterned micropolarizer array for cmos image sensors," IEEE Photonics Technology Letters, vol. 21, no. 12, pp. 805-807, 2009.

[3.14] V. Gruev, J. V. der Spiegel, and N. Engheta, "Image sensor with focal plane polarization sensitivity," Proceedings of International Symposium on Circuits and Systems, pp. 1028-1031, 2008.

[3.15] S. Faris, "Methods for manufacturing micropolarizers,u.s. patent 5,327,285," 1994.

[3.16] J. Guo and D. Brady, "Fabrication of thin-film micropolarizer arrays for visible imaging polarimetry," Applied Optics, vol. 39, no. 10, pp. 14861492, 2000.

[3.17] V. Gruev, A. Ortu, N. Lazarus, J. V. der Spiegel, and N. Engheta, "Fabrication of a dual-tier thin film micropolarization array," Optical Express, vol. 15, no. 8, pp. 4994-5007, 2007.

[3.18] H. Hertz, "Ueber die einwirkung einer geradlinigen electrischen schwingung auf eine benachbarte strombahn," Annalen der Physics, vol. 270, no. 5, pp. 155-170, 1888.

[3.19] G. Nordin, J. Meier, P. Deguzman, and M. Jones, "Micropolarizer array for infrared imaging polarimetry," Journal of Optical Society of America, A, vol. 16, pp. 1168-1174, 1999.

[3.20] T. Tokuda, H. Yamada, H. Shimohata, K. Sasagawa, and J. Ohta, "Polarization-analyzing cmos image sensor with embedded wire-grid polarizer," Proceedings of International Image Sensor Workshop, 2009.

[3.21] T. Tokuda, H. Yamada, K. Sasagawa, and J. Ohta, "Polarizationanalyzing cmos image sensor with monolithically embedded polarizer for microchemistry systems," IEEE Transactions on Biomedical Circuits and Systems, vol. 3, no. 5, pp. 259-266, 2009. 
[3.22] J. Wang, F. Walters, X. Liu, P. Sciortino, and X. Deng, "Highperformance, large area, deep ultraviolet to infrared polarizers based on 40nm line/78nm space nanowire-grids," Applied Physics Letters, vol. 90, pp. 061104-1-3, 2007.

[3.23] H. Lamb, "On the reflection and transmission of electric waves by a metallic grating," Proceedings of London Mathematical Society, vol. 29, no. 1, pp. 523-546, 1898.

[3.24] D. Malacara, Physical optics and light measurements, vol. 26, p. 157. Academic Press, 1989. ISBN:0124759718.

[3.25] E. Collett, Field Guide to Polarization, p. 25. Bellingham, WA: SPIE Press, 2005. ISBN: 9780819458681.

[3.26] V. Gruev, R. Perkins, and T. York, "Ccd polarization imaging sensor with aluminum nanowire optical filters," Optics Express, vol. 18, pp. 1908719094, 2010.

[3.27] X. Zhao, A. Bermak, F. Boussaid, and V. Chigrinov, "Liquid-crytal micropolarimeter array for visible linear and circular polarization imaging," Proceedings of International Symposium on Circuits and Systems, pp. 637640, 2009.

[3.28] M. Sarkar, D. S. Segundo, C. van Hoof, and A. J. P. Theuwissen, "Polarization analyzing cmos image sensor," Proceedings of International Symposium on Circuits and Systems, pp. 621-624, 2010.

[3.29] X. Zhao, A. Bermak, and F. Boussaid, "A cmos digital pixel sensor with photopatterened micropolarizer array for real time focal plane polarization imaging," Proceedings of IEEE Biomedical Circuits and Systems Conference, pp. 145-148, 2008.

[3.30] M. Guillaumee, L. Dunbar, C. Santschi, E. Grenet, R. Eckert, O. Martin, and R. Stanley, "Polarization sensitive silicon photodiodes using nanostructured metallic grids," Applied Physics Letters, vol. 94, pp. 193503-1-3, 2009.

[3.31] P. Catrysse and B. Wandell, "Integrated color pixels in $0.18 \mu \mathrm{m}$ complementary metal oxide semiconductor technology," Journal of Optical Society of America A, vol. 20, no. 12, pp. 2293-2306, 2003.

[3.32] P. Catrysse, B. Wandell, and A. E. Gamal, "An integrated color pixel in $0.18 \mu \mathrm{m}$ cmos technology," Technical digest of IEEE International Electron Device Meeting, pp. 24.4.4-24.4.4, 2001. 
[3.33] D. Goldstein, D. Chenault, and J. Pezzaniti, "Polarimetric characterization of spectralon," Polarization An integrated color pixel in $0.18 \mu \mathrm{m}$ CMOS technology: Proceedings of Measurement, Analysis, and Remote Sensing II SPIE, vol. 3754, pp. 126-136, 1999. 


\section{4}

\section{Material classification using CMOS polarization sensor}

Material classification is an important application in computer vision. The ability to detect the nature of the object surface from image data has a very high potential for applications ranging from low-level inspection to high-level object recognition. The inherent property of materials to partially polarize the reflected light can serve as a tool to classify them.

In this chapter, an introduction to material classification is presented in section 4.1. The theory behind polarization upon specular reflection and Fresnel coefficients is covered in section 4.2. The Fresnel reflectance model is also briefly described. The polarized nature of the transmitted light can be used to differentiate between metal and dielectric surfaces in real time due to the different nature in partially polarizing the specular and diffuse reflection components of the reflected light. This is elaborated in section 4.3 by measuring the transmitted irradiance after reflection from the material surface which allows computation of Fresnel reflection coefficients, the degree of partial polarization and the variations in the maximum and minimum transmitted intensities for varying specular angle of incidence. Differences in the physical parameters for various metal surfaces result in different surface reflection behavior, influencing the Fresnel reflection coefficients. Section 4.4, shows that it is possible to differentiate among various metals of varying conductivity by sensing the change in the Polarization Fresnel Ratio and the degree of polarization of the light reflected. Section 4.5 presents a short summary of the methods used to differentiate among material surfaces. 


\subsection{Introduction}

Materials can be broadly classified into metals and dielectrics based on their conductivity. Metals are highly conductive, opaque and tend to be very reflective while dielectrics are less conductive and have very low reflectivity.

Earlier attempts to distinguish between metals and dielectrics used the dichromatic reflection properties of the material surface [4.1], [4.2], [4.3]. Materials were classified into optically homogenous or optically inhomogeneous categories. Healey [4.4] showed that homogenous materials reflect light only from the surface. Thus, when illuminated with a monochromatic light beam the reflected light color would be nearly constant. Inhomogeneous materials on the other hand reflect light from the surface and also scatter light from the body. Such a material, when illuminated with a monochromatic light beam would reflect two distinct colors as the color of the surface reflected light will be different from the color of the scattered light from the body.

Once the object has been determined to be either homogenous or inhomogeneous, it can be further classified as a metal or a dielectric depending on the reflected color. Metals have free electrons, and the behavior of these free electrons when hit by an incident light ray is a function of its wavelength. Metals such as steel and nickel have nearly the same response over the entire visible spectrum, while metals such as copper and gold tend to reflect longer wavelengths more than the shorter ones. Dielectrics on the other hand do not have free electrons thus the reflectivity from a dielectric surface is independent of the wavelength of the incident light ray. This method, however, is limited by the distortion of the color histogram obtained after reflection, which depends on the object geometry. There is also speculation whether a definitive relationship exists between the reflected color distribution and the intrinsic composition of the surface. Additionally, the color of the material also influences the reflected color.

Another method to discriminate between metals and dielectrics based on the Fresnel reflection theory was proposed by Wolff [4.5], [4.6]. An unpolarized electromagnetic wave can be polarized upon specular reflection as described in chapter 3 . The polarization by reflection from a material surface depends on the angle at which the light strikes the reflecting surface as well as on the nature of said surface. Metallic surfaces reflect light with a variety of vibration directions, and such reflected light is usually unpolarized. However, non-metallic surfaces reflect light such that the vibrations of the reflecting light wave is parallel to the plane of the reflecting surface.

According to the Fresnel reflection theory, dielectric surfaces polarize the light 
upon specular reflection stronger than metal surfaces for all angles of incidence. Traditionally the polarization components of the transmitted irradiances are obtained by allowing the reflected light from the material surface to pass through an external linear polarizer onto a CCD or CMOS image sensor. The disadvantage of such a system is that the linear polarization filters have to be externally controlled, which complicates the automation and miniaturization of optical sensors for material classification. Further the digital processing required to process the obtained polarization information increases the complexity and power consumption of the system.

The model proposed by Wolff to attempt to classify materials based on the polarization information using the embedded wire grid metallic micro-polarizer presented in chapter 3 is used in this thesis. The metallic wire grid micropolarizer can be used to measure the transmitted irradiances after specular reflection from the material surface in real time. The metal grid oriented at $0^{\circ}$ and $90^{\circ}$ in the sense regions 1 and 2 allows us to measure the parallel and perpendicular Fresnel reflection coefficients and the maximum and the minimum transmitted irradiance after reflection from the material surface. The difference between the maximum and the minimum transmitted radiances relates to the degree of polarization at the surface [4.7]. Additionally, the degree of polarization based on the Stokes parameters discussed in chapter 3 is evaluated to find the relative difference between metals and dielectrics.

\subsection{Polarization and Fresnel coefficients}

When a light ray strikes a surface, part of the incident light is reflected and part is transmitted or absorbed as shown in figure 4.1. $\varphi$ is the angle of incidence of the incident ray, $\varphi$ ' is the angle of reflection of the reflected ray and $\eta$ is the angle of transmission of the transmitted ray.

The incident ray and the reflected ray are related by the law of reflection:

$$
\varphi=\varphi^{\prime}
$$

While the incident ray and the transmitted rays are related by the law of refraction or Snell's law:

$$
\epsilon_{1} \sin \varphi=\epsilon_{1} \sin \eta
$$

where $\epsilon_{1}$ is the refractive index of the incident medium and $\epsilon_{2}$ is the refractive 


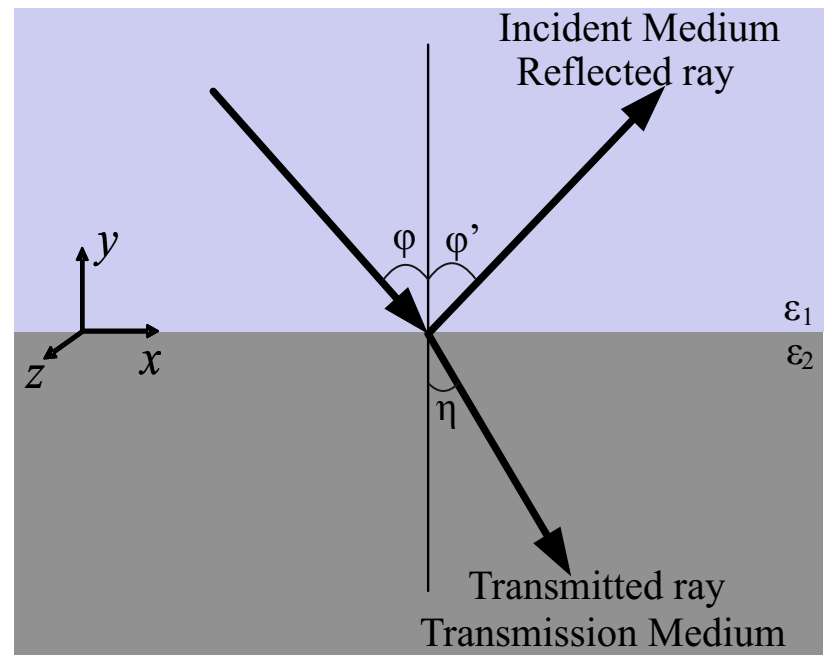

Figure 4.1: Incident light ray behavior at an interface.

index of the transmission medium.

The reflection occurring at the surface of the planar surface has two components: diffuse reflection and specular reflection, as shown in the figure 4.2.

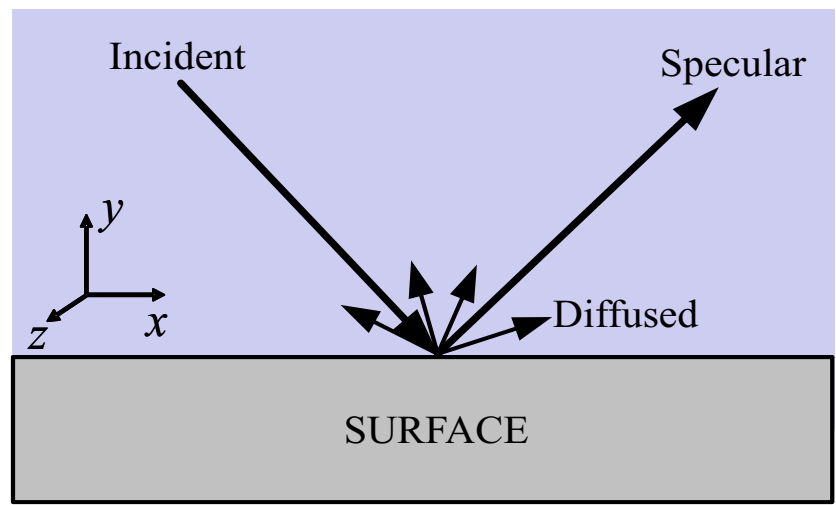

Figure 4.2: Reflection types: specular and diffuse reflections.

Diffuse reflection is caused by the reflected rays from internal scattering inside the surface of the medium. When light strikes a surface interface, a part of the light passes through the boundary. This light is re-emitted randomly after suffering internal scattering. This re-emitted light is known as diffuse reflection 
and is modeled by Lambert's equation:

$$
\begin{aligned}
I_{d} & =\left(C_{d} \times \vec{N}\right) \cdot \vec{S} \\
& =\left(C_{d} \times \cos \theta_{i}\right)
\end{aligned}
$$

where $I_{d}$ corresponds to the brightness $C_{d}$ is a proportionality constant, $\vec{N}$ is the surface orientation, $\vec{S}$ is the light source direction and $\theta_{i}$ (like $\varphi$ in figure 4.1) is the angle between the light source direction and the surface orientation. The diffuse reflection component is independent of the angle of reflection but depends on the angle of incidence.

Specular reflection is a mirror like reflection from a surface in which light from a single incoming direction is reflected into a single outgoing direction. Pure specular reflection occurs when the planar interface portion of the surface is significantly larger than the wavelength of the incident light [4.5]. The incident and the reflected directions of the specularly reflected light determine the specular plane of incidence (or simply specular plane) as shown in figure 4.3.

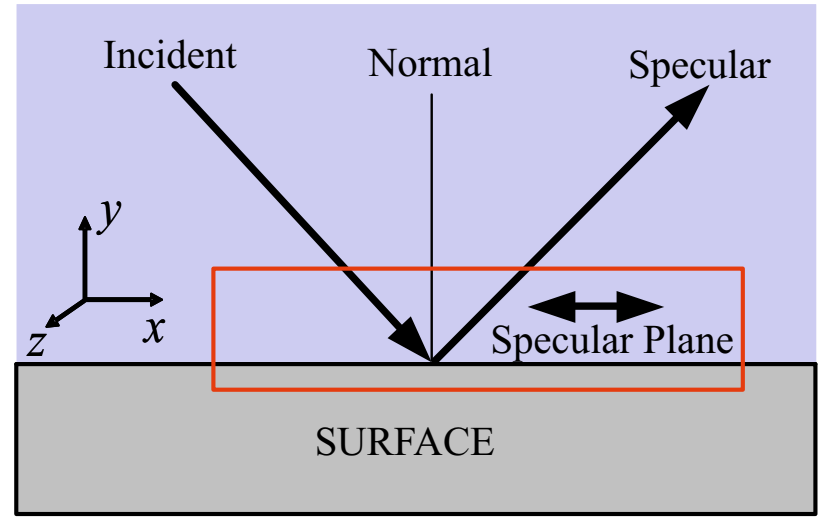

Figure 4.3: Specular reflection plane is parallel to $x$-y plane.

\subsubsection{Polarization properties of a reflected light}

Unpolarized light becomes partially polarized after specular reflection as shown in figure 4.4. In figure 4.4 the plane of incidence for a plane wave is $x z$, the plane of the two mediums is $x y$ and the angle of incidence is $\varphi$. For the reflected light, the intensity of the polarization component perpendicular to the specular plane is larger than the intensity of the polarization component parallel 
to the specular plane.

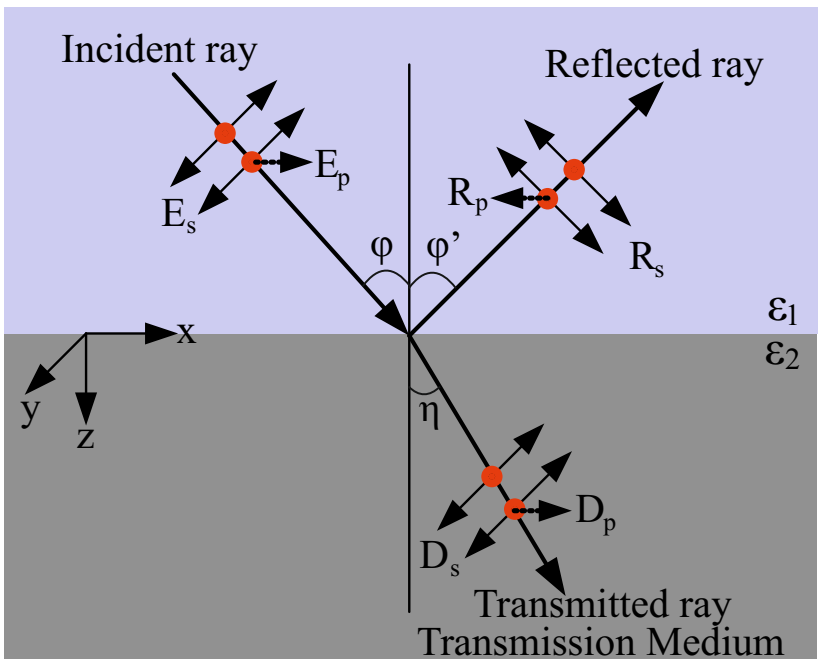

Figure 4.4: Incident, reflected and transmitted Fresnel coefficients.

A planar wave is represented by the equations:

$$
\begin{aligned}
& X=A_{x} \cos \frac{2 \pi}{T}\left(t-\frac{m x+n y+p z}{V}\right) \\
& Y=A_{y} \cos \frac{2 \pi}{T}\left(t-\frac{m x+n y+p z}{V}\right) \\
& Z=A_{z} \cos \frac{2 \pi}{T}\left(t-\frac{m x+n y+p z}{V}\right)
\end{aligned}
$$

where $A_{x}, A_{y}$ and $A_{z}$ are the components of the amplitude of the resultant electric forces in the $x, y$ and $z$ directions, respectively; $V$ is the velocity of propagation; $m, n$ and $z$ are the direction cosines of the normal to the wave front and fulfill the equation $m^{2}+n^{2}+p^{2}=1 ; T$ is the time period of the wave and $t$ is the time independent variable [4.8].

The phase in equation (4.4) is the same for all planes thus the wave fronts can be written as:

$$
m x+n y+p z=\text { constant }
$$

The amplitudes of the resultant electric forces, $A_{x}, A_{y}$ and $A_{z}$ are proportional 
to the directional cosines and thus satisfy equation (4.6).

$$
\left(A_{x} \times m\right)+\left(A_{y} \times n\right)+\left(A_{z} \times p\right)=0
$$

The direction cosines of the direction of propagation of the incident wave in figure 4.4 are then expressed as:

$$
\begin{aligned}
m & =\sin \varphi \\
n & =0 \\
p & =\cos \varphi
\end{aligned}
$$

The incident electric field can be resolved into two components, one perpendicular to the plane of incidence with amplitude $E_{s}$ and the other parallel to the plane of incidence or in the plane of incidence with amplitude $E_{p}$. The first component is parallel to the $y$-axis and can be expressed using equation (4.4) and (4.7) as:

$$
Y_{e}=E_{s} \cos \frac{2 \pi}{T}\left(t-\frac{x \sin \varphi+z \cos \varphi}{V_{1}}\right)
$$

$V_{1}$ is the velocity of the light in the incident medium:

$$
V_{1}=\frac{c}{\sqrt{\epsilon_{1}}}
$$

where, $c$ is the speed of light and $\epsilon_{1}$ is the index of refraction of the incident medium.

The incident wave is transverse in nature with an electric force $E_{p}$ perpendicular to the plane of incidence, thus the components $A_{x}$ and $A_{z}$ in the $x$ and $z$ directions must have the values:

$$
\begin{aligned}
& A_{x}=E_{p} \cos \varphi \\
& A_{z}=-E_{p} \sin \varphi
\end{aligned}
$$

The $x$ and $z$ components of the electrical force of the incident wave are then expressed as: 


$$
\begin{aligned}
& X_{e}=E_{p} \cos \varphi \cos \frac{2 \pi}{T}\left(t-\frac{x \sin \varphi+z \cos \varphi}{V_{1}}\right) \\
& Z_{e}=-E_{p} \sin \varphi \cos \frac{2 \pi}{T}\left(t-\frac{x \sin \varphi+z \cos \varphi}{V_{1}}\right)
\end{aligned}
$$

When the incident transverse wave reaches the boundary it is divided into a reflected and a refracted wave component. The electric forces in the reflected wave are:

$$
\begin{aligned}
X_{r} & =R_{p} \cos \varphi^{\prime} \cos \frac{2 \pi}{T}\left(t-\frac{x \sin \varphi^{\prime}+z \cos \varphi^{\prime}}{V_{1}}\right) \\
Y_{r} & =R_{s} \cos \frac{2 \pi}{T}\left(t-\frac{x \sin \varphi^{\prime}+z \cos \varphi^{\prime}}{V_{1}}\right) \\
Z_{r} & =-R_{p} \sin \varphi^{\prime} \cos \frac{2 \pi}{T}\left(t-\frac{x \sin \varphi^{\prime}+z \cos \varphi^{\prime}}{V_{1}}\right)
\end{aligned}
$$

And the electric forces in the refracted wave are:

$$
\begin{aligned}
X_{2} & =D_{p} \cos \eta \cos \frac{2 \pi}{T}\left(t-\frac{x \sin \eta+z \cos \eta}{V_{2}}\right) \\
Y_{2} & =D_{s} \cos \frac{2 \pi}{T}\left(t-\frac{x \sin \eta+z \cos \eta}{V_{2}}\right) \\
Z_{2} & =-D_{p} \sin \eta \cos \frac{2 \pi}{T}\left(t-\frac{x \sin \eta+z \cos \eta}{V_{2}}\right)
\end{aligned}
$$

where $R_{p}, R_{s}, D_{p}$ and $D_{s}$ are the amplitudes of reflected and refracted electrical forces, $\varphi^{\prime}$ is the angle of reflection, $\eta$ is the angle of refraction and $V_{1}$ and $V_{2}$ are the velocity of the wave in the incident and the transmission medium respectively.

The boundary condition states that the component of the electric and magnetic forces parallel to the surface must vary continuously in passing through the transition layer. From equations (4.8), (4.11), (4.12) and (4.13) and using the law of reflection from equation (4.1) we obtain: 


$$
\begin{aligned}
\left(E_{p}-R_{p}\right) \cos \varphi & =D_{p} \cos \eta \\
\left(E_{s}+R_{s}\right) & =D_{s} \\
\left(E_{s}-R_{s}\right) \sqrt{\epsilon_{1}} \cos \varphi & =D_{s} \sqrt{\epsilon_{2}} \cos \eta \\
\left(E_{p}+R_{p}\right) \sqrt{\epsilon_{1}} & =D_{p} \sqrt{\epsilon_{2}}
\end{aligned}
$$

From the above equations the amplitude of the reflected and refracted amplitudes can be calculated in terms of the incident amplitude as:

$$
\begin{aligned}
2 E_{s} & =D_{s}\left(1+\frac{\sqrt{\epsilon_{2}} \cos \eta}{\sqrt{\epsilon_{1}} \cos \varphi}\right) \\
E_{s}\left(\frac{\sqrt{\epsilon_{1}} \cos \varphi}{\sqrt{\epsilon_{2}} \cos \eta}-1\right) & =R_{s}\left(\frac{\sqrt{\epsilon_{1}} \cos \varphi}{\sqrt{\epsilon_{2}} \cos \eta}+1\right) \\
2 E_{p} & =D_{p}\left(\frac{\cos \eta}{\cos \varphi}+\frac{\sqrt{\epsilon_{2}}}{\sqrt{\epsilon_{1}}}\right) \\
E_{p}\left(\frac{\cos \varphi}{\cos \eta}-\frac{\sqrt{\epsilon_{1}}}{\sqrt{\epsilon_{2}}}\right) & =R_{p}\left(\frac{\cos \varphi}{\cos \eta}+\frac{\sqrt{\epsilon_{1}}}{\sqrt{\epsilon_{2}}}\right)
\end{aligned}
$$

From the laws of refraction:

$$
\sqrt{\epsilon_{1}} \sin \eta=\sqrt{\epsilon_{2}}
$$

From equations (4.15) and (4.16) the Fresnel reflection equations can be derived:

$$
\begin{aligned}
R_{s} & =-E_{s} \frac{\sin (\varphi-\eta)}{\sin (\varphi+\eta)} \\
R_{p} & =-E_{p} \frac{\tan (\varphi-\eta)}{\tan (\varphi+\eta)} \\
D_{s} & =-E_{s} \frac{2 \sin \eta \cos \varphi}{\sin (\varphi+\eta)} \\
D_{p} & =-E_{p} \frac{2 \sin \eta \cos \varphi}{\sin (\varphi+\eta) \cos (\varphi-\eta)}
\end{aligned}
$$

where $R_{s}$ and $R_{p}$ represent the perpendicular and parallel Fresnel coefficients for reflection with $D_{s}$ and $D_{p}$ are the perpendicular and parallel Fresnel coefficients for transmission of the incident light ray. 
From equations (4.17) it can be said that for any given incident ray there exists a state of polarization in the reflected and transmitted ray. The state of polarization of a light wave describes the electric field magnitude and phase relationships inside the reflected and the transmitted wave.

\subsubsection{Fresnel reflectance model}

The Fresnel reflectance model describes the intensity and spectral composition of the light reflected from the reflection surface and reaching the observer. The intensity of the reflected light depends on the intensity of the light source and also on the surface properties of the material. The spectral composition of the reflected light is determined by the wavelength selective reflection of the surface. In this section fresnel reflection coefficients are determined from the spectral composition of the reflected light which is further used to define polarization Fresnel ratio $(P F R)$.

When the reflected wave component is passed through a linear polarizer, the intensity of the image can be expressed as a function of the transmission axis of the polarizer $(\theta)$ [4.5]. The spectral transmittances of the polarizer for linearly polarized light and unpolarized light are denoted by $T_{p}(\lambda)$ and $T_{n}(\lambda)$ respectively, where $\lambda$ is the wavelength of the light.

The intensity image of the reflected light from the surface is expressed in terms of the pixel coordinates $(x, y)$ and the transmission axis of the polarizer. The intensity image obtained after the reflection can be written as a sum of the diffuse reflection coefficient $I_{d}(x, y)$ and the specular reflection coefficient $I_{s}(x, y)$ [4.5],[4.7]. Using the Fresnel reflection equations (4.17), the intensity image observed through the polarizer transmission axis $\theta$ can be expressed as:

$$
\begin{aligned}
I(x, y: \theta)= & T_{n} I_{d}(x, y)+T_{p} I_{s}(x, y) \\
& \times \frac{R_{p}(x, y) \sin ^{2}\left(\theta-\theta_{0}\right)+R_{s}(x, y) \cos ^{2}\left(\theta-\theta_{0}\right)}{R_{p}(x, y)+R_{s}(x, y)}
\end{aligned}
$$

where $\theta_{0}$ is the direction perpendicular to the specular plane. The Fresnel reflection coefficients $R_{p}$ and $R_{s}$ depend on the pixel coordinates. In terms of polarized and unpolarized components, equation (4.18) can be rewritten as:

$$
I(x, y: \theta)=A(x, y) \frac{1+\cos \left(2\left(\theta-\theta_{0}\right)\right)}{2}+B(x, y)
$$


where:

$$
\begin{aligned}
& A(x, y)=\frac{R_{p}(x, y)-R_{s}(x, y)}{R_{p}(x, y)+R_{s}(x, y)} T_{p} I_{s}(x, y) \\
& B(x, y)=\frac{R_{p}(x, y)}{R_{p}(x, y)+R_{s}(x, y)} T_{p} I_{s}(x, y)+T_{n} I_{d}(x, y)
\end{aligned}
$$

The first term in equation (4.19) is the polarized component of the reflection mainly contributed by the specular reflection of the incident transverse wave. The second term represents the unpolarized component contributed by the diffuse reflections and partly by the specular reflection which is not polarized.

It can be observed from equation (4.19) that $I(x, y: \theta)$ oscillates as $\theta$ varies between the maximum $I_{\max }(x, y)$ at $\theta=\theta_{0}, \theta_{0}+\pi$ and the minimum $I_{\min }(x, y)$ at $\theta=\theta_{0} \pm \pi / 2 . \quad I_{\max }(x, y)$ and $I_{\min }(x, y)$ are then expressed in terms of equations (4.20) and (4.21) as:

$$
\begin{aligned}
I_{\text {max }}(x, y) & =A(x, y)+B(x, y) \\
& =\frac{R_{s}(x, y)}{R_{p}(x, y)+R_{s}(x, y)} \times T_{p} I_{s}(x, y)+T_{n} I_{d}(x, y) \\
I_{\text {min }}(x, y) & =B(x, y) \\
& =\frac{R_{p s}(x, y)}{R_{p}(x, y)+R_{s}(x, y)} \times T_{p} I_{s}(x, y)+T_{n} I_{d}(x, y)
\end{aligned}
$$

The Polarization Fresnel Ratio $(P F R)$ is the ratio of the perpendicular Fresnel coefficient to the parallel Fresnel coefficient [4.5]. The Fresnel reflection and transmission coefficients in the Fresnel reflectance model are given by equation (4.17).

The PFR can be derived by rearranging the equations (4.22) and (4.23).

$$
\begin{aligned}
& R_{s}(x, y)=\frac{\left[I_{\max }(x, y)-T_{n} I_{d}(x, y)\right]\left[R_{p}(x, y)+R_{s}(x, y)\right]}{T_{p} I_{s}(x, y)} \\
& R_{p}(x, y)=\frac{\left[I_{\min }(x, y)-T_{n} I_{d}(x, y)\right]\left[R_{p}(x, y)+R_{s}(x, y)\right]}{T_{p} I_{s}(x, y)}
\end{aligned}
$$


Dividing equations (4.24) and (4.25), we get

$$
P F R=\frac{R_{s}(x, y)}{R_{p}(x, y)}=\frac{\left[I_{\max }(x, y)-T_{n} I_{d}(x, y)\right]}{\left[I_{\min }(x, y)-T_{n} I_{d}(x, y)\right]}
$$

The specular component of reflection in metals is greater than the diffuse component of reflection $I_{s}(x, y) \gg I_{d}(x, y)$. Thus the diffuse component of reflection $I_{d}(x, y)$ in equation (4.26) can be neglected resulting in equation (4.27).

$$
P F R=\frac{R_{s}(x, y)}{R_{p}(x, y)}=\frac{\left[I_{\max }(x, y)\right]}{\left[I_{\min }(x, y)\right]}
$$

Equation (4.27) is the polarization Fresnel ratio which is related to the ratio of the maximum to minimum transmitted irradiance detected after specular reflection from the material surface.

In the case of dielectrics the Fresnel reflection coefficient $R_{p}$ is very small for all specular angles of incidence, and is almost zero near the Brewster angle. The $P F R$ for dielectrics thus can be arbitrary large as predicted by equation (4.27). For dielectrics with specular angle of incidence very close to the Brewster angle, equation (4.27) becomes

$$
\frac{R_{s}(x, y)}{R_{p}(x, y)} \gg \frac{\left[I_{\max }(x, y)\right]}{\left[I_{\min }(x, y)\right]}
$$

\subsection{Material classification measurements}

In the section 4.2, it was shown that the reflected and transmitted light polarization components are related to the incident polarization components. The reflected polarization components are dependent on the angle of incidence and the nature of the material surface. In this section the variation in the polarization patterns for two materials, metal and plastic, are experimentally detected. The varying oscillations of the transmitted intensities, the degree of polarization and the PFR obtained from the polarization component of the reflected light for both the material surface are evaluated. 


\subsubsection{Measurement setup}

The measurement setup for the measurements of the maximum and minimum transmitted intensities after reflection from the material surface is shown in figure 4.5.

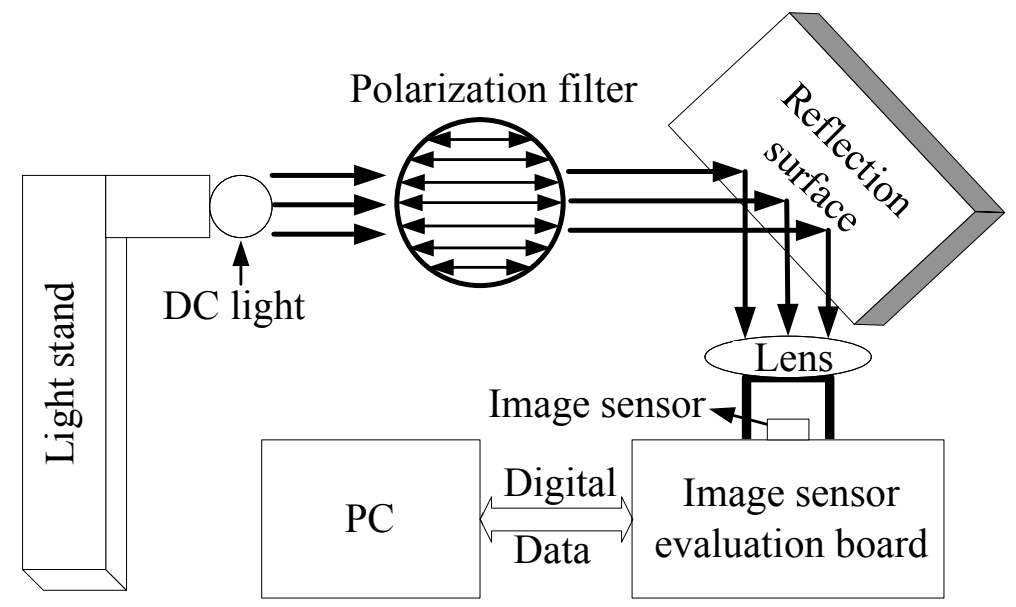

Figure 4.5: Measurement setup for at $\lambda=650 \mathrm{~nm}$.

The light from the DC light source (similar to one used in 3.12 ) is completely unpolarized. The linear polarization filter allows only those amplitudes which are parallel to the transmission axis of the polarization filter to pass through. The polarized electromagnetic waves are reflected from the surface. At the boundary of the reflection surface both the diffuse component and the specular component of the reflection of the incident light are present. These reflection components are then focused on the image sensor by the lens. The analog signal from the image sensor is digitized using an external $A D C$ and then analyzed using a PC as described in section 2.6.1.

\subsubsection{Polarization transmittance}

The magnitude of variations of the reflected irradiance of the light after reflection is larger for dielectrics than for metals [4.5]. The theoretically expected image intensity $I(x, y: \theta)$ of the transmitted radiance of light reflected from the dielectric and metal surfaces is shown in figure 4.6 [4.7].

The intensity of the reflected wave is a function of the transmission axis of the linear polarizer $I(x, y: \theta)$ and oscillates between the maximum trans- 


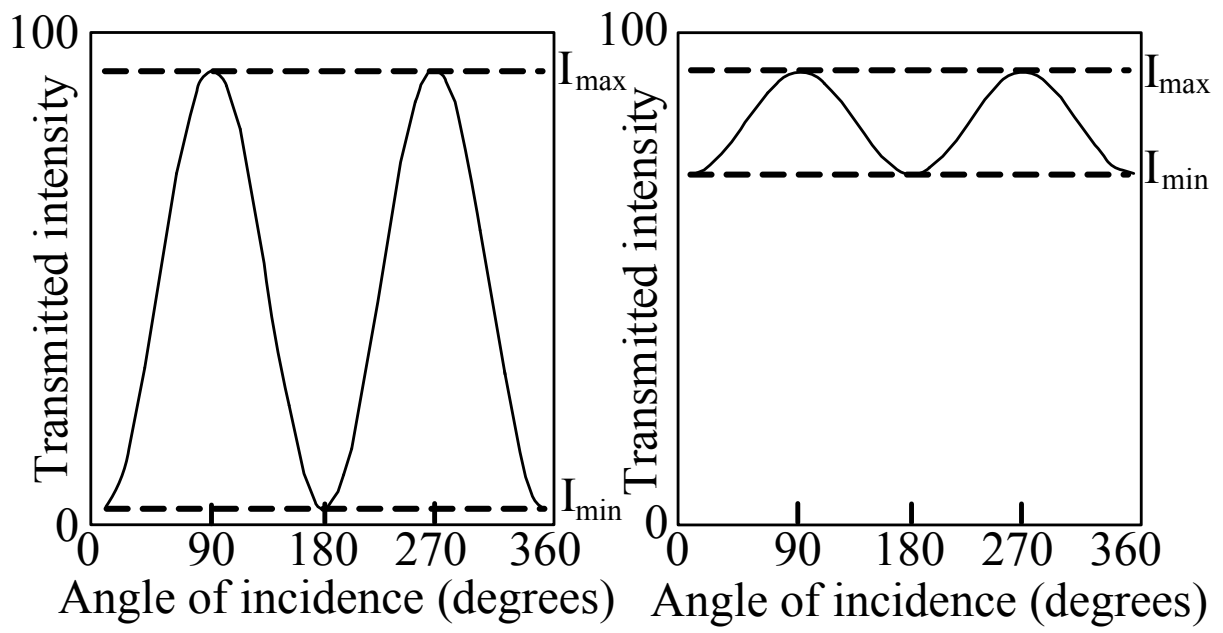

Figure 4.6: Transmitted radiance of reflected light as a function of transmission axis of the linear polarizer (left) dielectric surface (right) metal surface.

mitted intensity $I_{\max }$ and the minimum transmitted intensity $I_{\min }$ as shown in equation (4.19). For dielectrics the Fresnel coefficients satisfy $R p \gg R_{s}$ while for metals $R_{p} \approx R_{s}$. The diffuse component of reflection dominates over the specular component $I_{d}(x, y) \gg I_{s}(x, y)$ for dielectrics and thus the oscillations given by equations (4.22) and (4.23) vary over a larger range, while in the case of metals, the oscillations are relatively smaller as the specular component of reflection dominates over the diffuse component of reflection $I_{s}(x, y) \gg I_{d}(x, y)$.

Figure 4.7 shows the measured transmitted irradiance in the sense regions 1 and 2 at $0^{\circ}$ and $90^{\circ}$ sensitive pixels for aluminum and plastic reflecting surface. The differences between the maximum and minimum transmitted irradiances for plastic and aluminum surfaces in both regions 1 and 2 are shown in table 4.1.

\begin{tabular}{|c|c|c|c|c|}
\hline & \multicolumn{2}{|c|}{ Region 1 } & \multicolumn{2}{c|}{ Region 2 } \\
\cline { 2 - 5 } & Plastic & Alum. & Plastic & Alum. \\
\hline$I_{\max }-I_{\min }\left(0^{\circ}\right)$ & 0.66 & 0.15 & 0.466 & 0.16 \\
\hline$I_{\max }-I_{\min }\left(90^{\circ}\right)$ & 0.67 & 0.23 & 0.985 & 0.10 \\
\hline
\end{tabular}

Table 4.1: Transmitted radiance for plastic and aluminum.

The range of the transmitted irradiance variation with incident polarization angle for plastic in sense region 1 is $190 \%$ and $340 \%$ higher than that of 


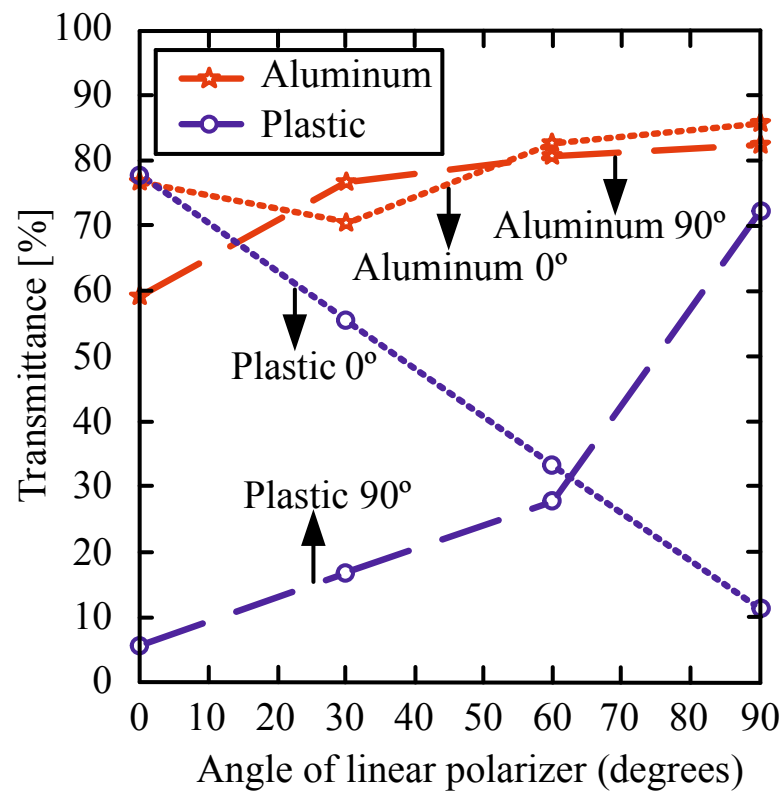

(a)

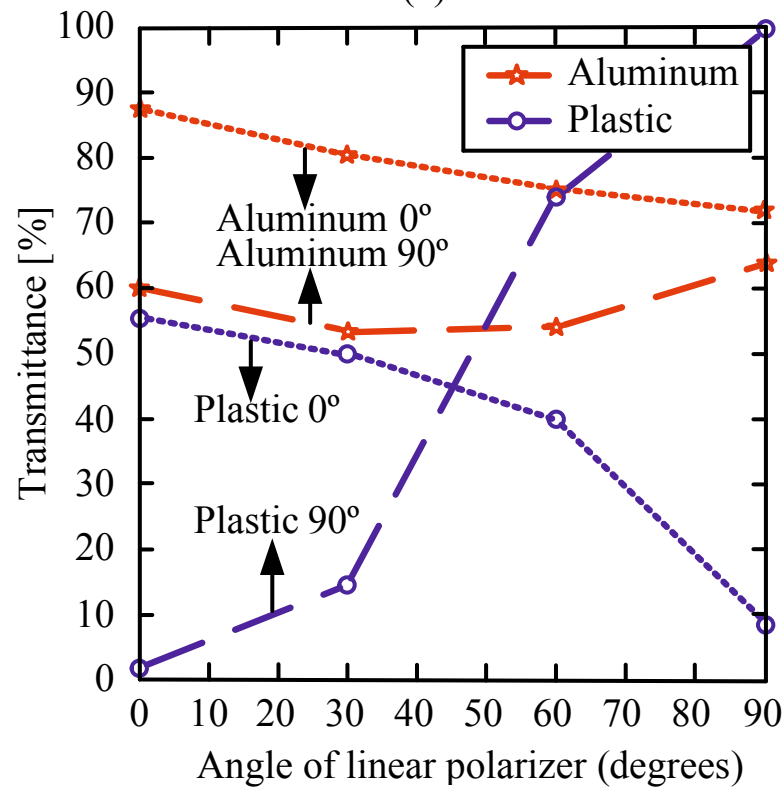

(b)

Figure 4.7: Transmitted intensity at 0 and 90 polarization sensitive pixel in (a) sense region $1(b)$ and sense region 2. 
aluminium. In sense region 2 the range of the oscillations for plastic is $190 \%$ and $885 \%$ higher than that of aluminum, for $90^{\circ}$ and $0^{\circ}$ polarization sensitive pixels respectively [4.9]. The transmitted irradiance oscillations for plastic at both polarization sense regions 1 and 2 are found to be much higher than those for aluminum. The differences in the transmitted irradiance are due to the difference in the reflection pattern of the light from the aluminum and plastic surfaces.

\subsubsection{Material classification using the degree of polariza- tion}

The light reflected from the material surface is partially polarized. The polarization state of the reflected light can thus be represented as a sum of a completely polarized component and a completely unpolarized component [4.5], [4.7]. The degree of polarization is the ratio of intensity of the perfectly polarized light reflected to the total intensity of the reflected light. The difference between the maximum and the minimum transmitted intensities given by equations (4.22) and (4.23) shows the amount of reflected light that is completely polarized. The minimum transmitted radiance $I_{\min }$ is one half of the magnitude of the unpolarized light reflected from the object surface. If $I_{p}(x, y)$ is the partial polarization component and $I_{a l l}(x, y)$ is the total reflected component then:

$$
\begin{aligned}
I_{p}(x, y) & =T_{p}^{-1} A(x, y)=T_{p}{ }^{-1}\left[I_{x}(x, y)-I_{\min }(x, y)\right] \\
I_{\text {all }}(x, y) & =I_{p}(x, y)+T_{n}{ }^{-1} B(x, y) \\
& =T_{p}^{-1}\left[I_{x}(x, y)-I_{\min }(x, y)\right]+2 T_{p}{ }^{-1} I_{\min }(x, y) \\
& =T_{p}{ }^{-1}\left[I_{x}(x, y)+I_{\min }(x, y)\right]
\end{aligned}
$$

And the degree of polarization is then obtained from equation (4.29):

$$
D O P=\frac{I_{p}(x, y)}{I_{\text {all }}(x, y)}=\frac{\left[I_{\max }(x, y)\right]-\left[I_{\min }(x, y)\right]}{\left[I_{\max }(x, y)\right]+\left[I_{\min }(x, y)\right]}
$$

Equation (4.30) also indicates the portion of the reflected light which is completely polarized to the total amount of reflected light, denoting the partial polarization [4.5]. Equation (4.30) has a maximum value of one and a minimum value of zero. At a value of zero the reflected light is completely unpolarized, thus the diffuse component of the reflection dominates over the specular component. At the maximum value of one, the reflected light is completely polarized, thus 
the specular component of the reflection dominates over the diffuse component.

The transmitted intensities are obtained using the measurement setup shown in figure 4.5 as explained in section 4.3.2. The degree of polarization obtained for the polarization sense regions 1 and 2 is shown in figure 4.8. In figure 4.8, a $D O P$ of 1 indicates the complete polarization of the reflected light and a $D O P$ of 0 indicates the completely unpolarized state of the reflected light.

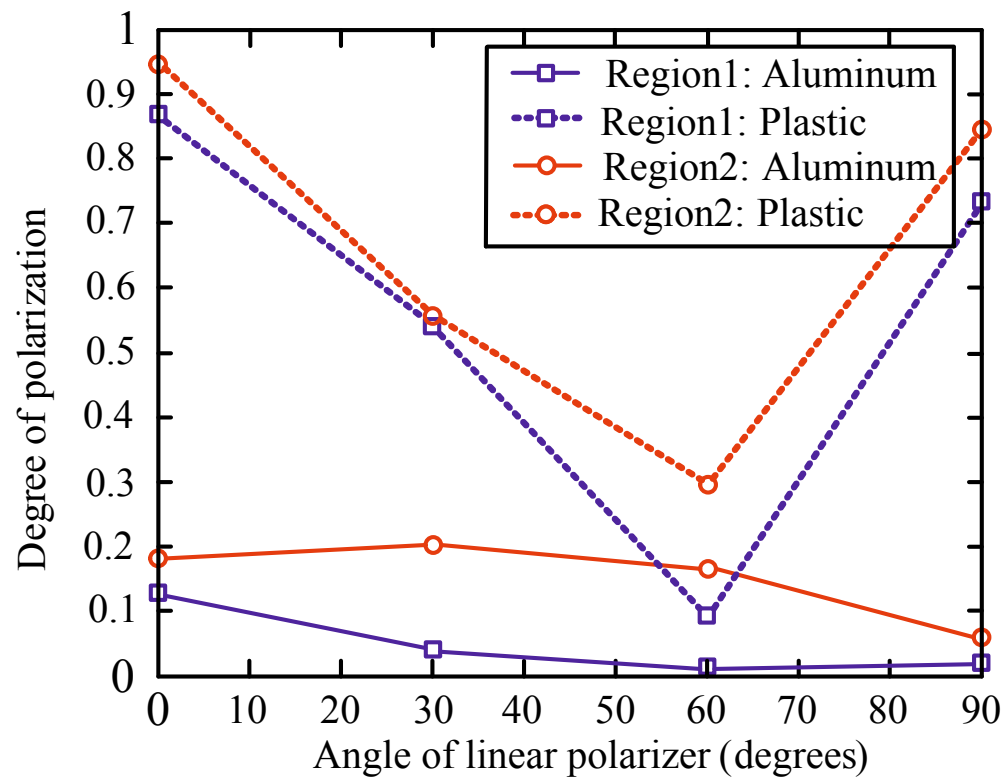

Figure 4.8: Degree of polarization in sense regions 1 and 2.

The maximum and minimum values of the $D O P$ for the two reflecting surfaces of plastic and aluminum in the two polarization sense regions 1 and 2 are given in table 4.2.

\begin{tabular}{|c|c|c|c|c|}
\hline & \multicolumn{2}{|c|}{ Region 1 } & \multicolumn{2}{c|}{ Region 2 } \\
\cline { 2 - 5 } & Plastic & Alum. & Plastic & Alum. \\
\hline DOP (Max) & 0.867 & 0.128 & 0.945 & 0.202 \\
\hline DOP (Min) & 0.09 & 0.02 & 0.298 & 0.06 \\
\hline
\end{tabular}

Table 4.2: Degree of polarization for plastic and aluminum.

The degree of polarization is higher for plastic than for aluminum. It is observed from figure 4.8 that for plastic the maximum $D O P$ is near one in 
both polarization sense regions, while for aluminum the maximum $D O P$ in both polarization sense regions is less than 0.2. A higher $D O P$ indicates higher amount of the reflected light being polarized, as stated in section 4.2.2.

It is further observed from figure 4.8 that as the specular angle of incidence is increased; the $D O P$ tends to decrease for both plastic and aluminum. However after a certain specular angle of incidence, the $D O P$ of aluminum continues to fall but the $D O P$ of the plastic shows a sharp rise. From equation (4.17) it is seen that $R_{s}$ never vanishes but $R_{p}$ becomes zero when:

$$
\begin{aligned}
\tan (\varphi+\eta) & =\infty \\
(\varphi+\eta) & =\frac{\pi}{2}
\end{aligned}
$$

From equation (4.31)

$$
\begin{aligned}
\sin \eta & =\sin \left(\frac{\pi}{2}-\varphi\right) \\
\tan \varphi & =n
\end{aligned}
$$

where $n$ is the index of refraction. When the angle of incidence satisfies the condition in equation (4.32), the electric field amplitude in the reflected wave has no component which lies in the plane of incidence, meaning that the entire component $R_{p}$ gets refracted. The only component in the reflected wave is the one that is perpendicular to the plane of incidence. The reflected wave is completely polarized. The angle of incidence $\varphi$ for which the reflected wave is completely polarized is known as the Brewster angle. The Brewster angle for plastics is near $60^{\circ}$ [4.10], which explains the sharp rise of the $D O P$ of plastic around $60^{\circ}$. For light absorbing material like metals $R_{p}$ is never 0 and thus no such sharp increase in the $D O P$ of aluminum is observed.

\subsubsection{Material classification using the Stokes parameters}

As described in chapter 3, the polarization state of an electromagnetic wave can conveniently be described by a set of Stokes parameters. To our best knowledge, there is no reference available on using Stokes parameters to classify materials. Here the degree of polarization and linear degree of polarization obtained from the Stokes vector to classify materials into metals and dielectrics are used.

The measurement setup is the same as shown in figure 4.5. The outputs of 
the pixels sensitive to $90^{\circ}$ and $0^{\circ}$ are averaged over 30 frames. Simultaneously, the output of the intensity sensitive pixels with no metal grid is also recorded for varying transmission axis of the external linear polarizer. The pixel output of the $0^{\circ}$ and $90^{\circ}$ polarization sensitive pixels are then normalized with the output of the intensity sensitive pixels. The normalized outputs correspond to $E_{x 0}$ and $E_{y 0}$ in equation (3.15). The degree of linear polarization is calculated using equation (3.17). The Stokes parameters $S_{0}$ and $S_{1}$ can be obtained using equation (3.15). The Stokes degree of linear polarization in the regions 1 and 2 are shown in figure 4.9 , where a 1 corresponds to a completely polarized state and a 0 indicates completely unpolarized state of the reflected light.

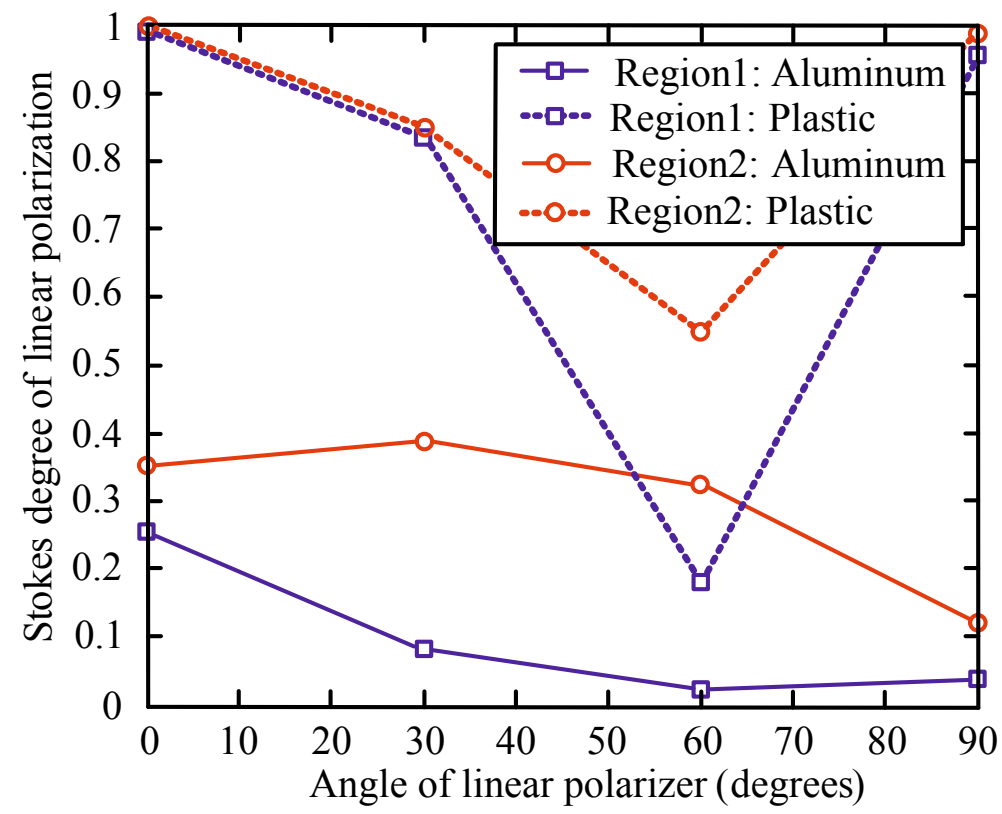

Figure 4.9: Stokes parameters, degree of linear polarization in sense region 1 and 2.

The Stokes degree of linear polarization for plastic has a maximum value of 1 and is higher than that for aluminum. It is further observed that the Stokes degree of linear polarization for plastics steadily decreases until it reaches the Brewster angle where the degree of polarization again increases to its maximum value. The maximum Stokes degree of linear polarization for aluminum obtained in the sense regions 1 and 2 are 0.25 and 0.38 respectively.

The behavior of the degree of polarization obtained using Stokes parameters 
(figure 4.9) are found to be very similar to the degree of polarization obtained using the maximum and minimum transmitted intensities (figure 4.8). It should indeed be the case as in linear polarization; the circular and elliptical forms of polarization are nearly absent and thus only the differences in maximum and minimum transmitted intensities are prevalent. In figure 4.8 , aluminum has a degree of polarization less than 0.2 in both regions for varying angle of linear polarizer, while in figure 4.9 the degree of polarization is less than 0.4 in both regions. While for plastic the $D O P$ is near one in both regions which steadily decreases till near Brewster angle and then further increases.

The above discussion was related to the degree of linear polarization obtained from the Stokes parameters. The Stokes degree of polarization is given by equation (3.16). The fourth Stokes parameter $S_{3}$ is usually ignored for natural light [4.11], since the phase information between orthogonally polarized light is difficult to calculate for natural light. Thus equation (3.16) can be modified with $S_{3}=0$ as

$$
D O P=\frac{\sqrt{S_{1}{ }^{2}+S_{2}{ }^{2}}}{S_{0}}
$$

$S_{0}$ and $S_{1}$ are the same as for the linear degree of polarization. $S_{2}$ is calculated from equations (3.15). The phase difference, $\delta$, in equation (3.15), is set to the specular angle of incidence. The obtained degrees of polarization in the polarization sense regions 1 and 2 are shown in figure 4.10.

In figure 4.10, a Stokes $D O P$ of 1 indicates the complete polarization of the reflected light while a 0 indicates the completely unpolarized state of the reflected light. Plastic shows a similar response to figure 4.9 while aluminum shows a steady decrease from the maximum value to minimum with increasing angle of linear polarizer. As the angle of specular incidence approaches the Brewster angle, the $D O P$ in case of plastics increases to its maximum value while no such behavior is observed for aluminum neither in sense region 1 nor in region 2.

A threshold can be applied to the Brewster angle to classify between metals and dielectric using the degree of polarization computed using the Stokes parameters. Near the Brewster angle the Stokes $D O P$ for plastics will be very near to 1 while in the case of metals it will be near 0 . 


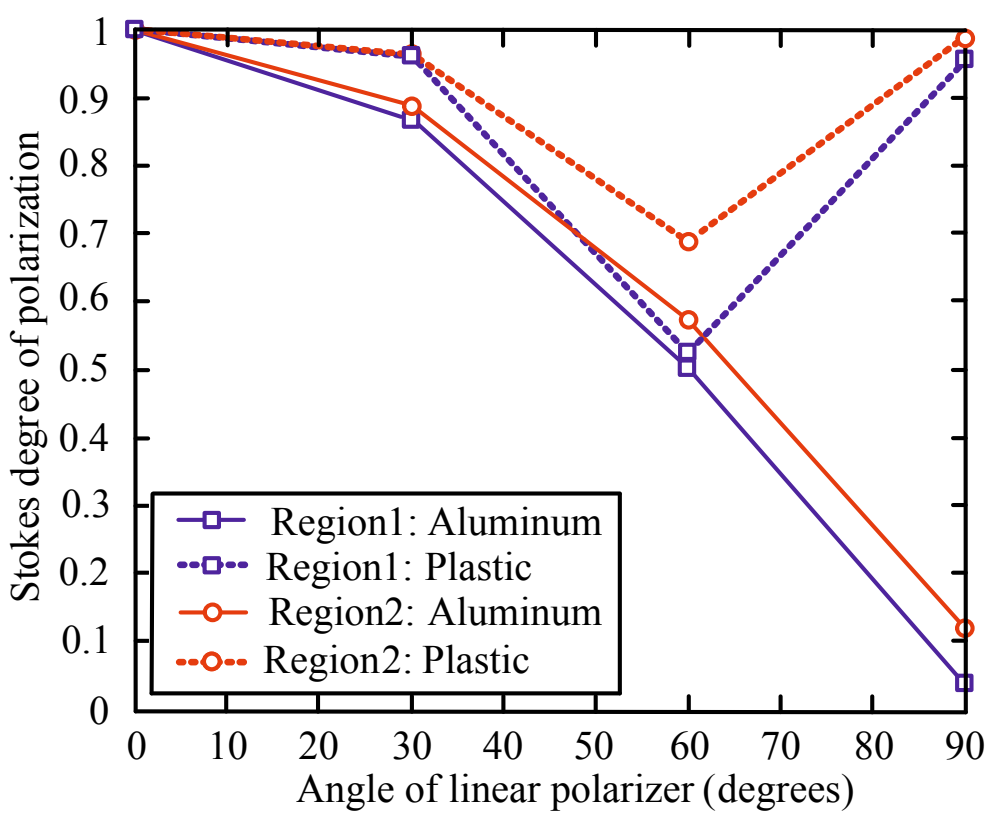

Figure 4.10: Stokes degree of polarization in sense region 1 and 2.

\subsubsection{Material classification using polarization Fresnel ra- tio}

Wolff in [4.5] introduces the polarization Fresnel ratio $(P F R)$ based on Fresnel reflectance model as a metric tool to classify materials into metals and dielectrics. The theoretical Fresnel reflection coefficients for aluminum and plastic are shown in figure 4.11 .

The Fresnel reflection coefficient for aluminum is near 1, while that for plastic varies over the entire span from 0 to 1 for different specular angles of incidence. The Fresnel reflection coefficient $R_{p}$ is 0 near the Brewster angle for plastic and thus the PFR for dielectric (plastic) can become arbitrarily large, while the PFR for metals is limited.

A material with significant conductivity will have a significantly reduced $P F R$ over a large range of specular angle of incidence. Since the conductivity of metals is higher than the conductivity of dielectrics, the PFR for metals is much smaller compared to that of dielectrics. As in metals the specular component of reflection is larger than the diffuse component of the reflection, the Fresnel coefficients satisfy $R_{s}(x, y) \approx R_{p}(x, y)$ [4.7]. Using this condition in equation (4.20) 


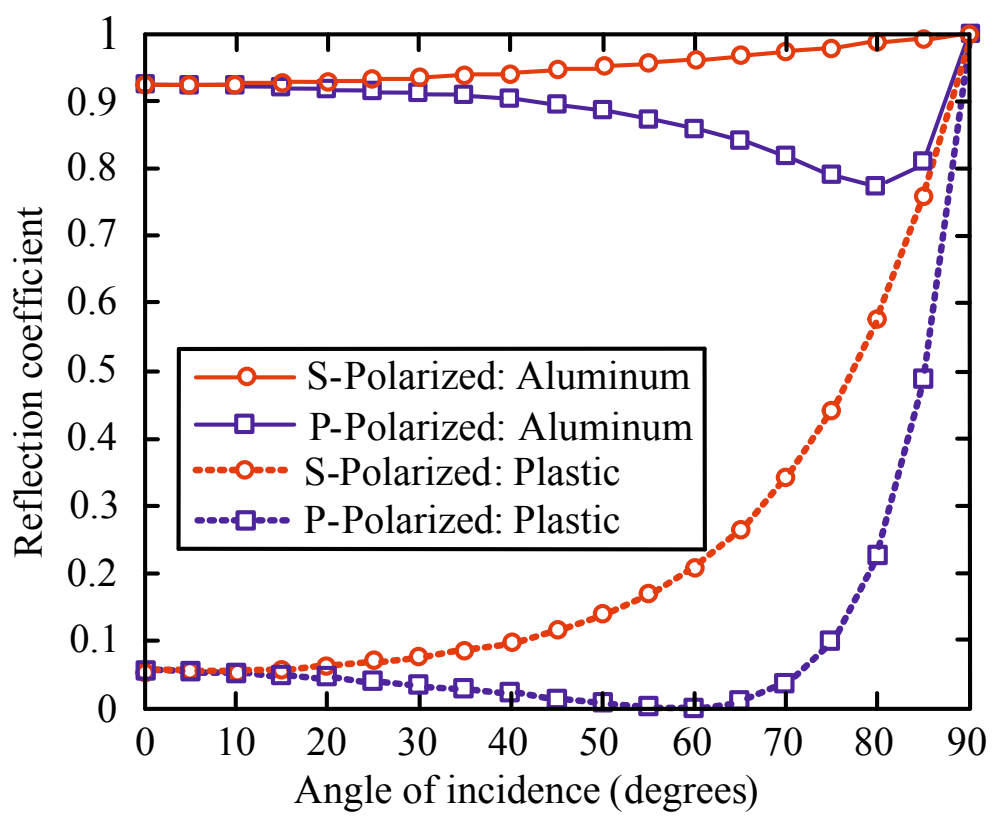

Figure 4.11: Theoretical Fresnel reflection coefficients for aluminum and plastic at $\lambda=500 \mathrm{~nm}$.

and (4.21) we get $A(x, y) \ll B(x, y)$ which from equation (4.22) and (4.23) means $I_{\max }(x, y) \approx I_{\min }(x, y)$, thus the PFR from equation (4.27) is nearly equal to 1 for all angles of incidence. The theoretical $P F R$ for metal and dielectric surface is shown in the figure 4.12 [4.5].

There exists a threshold in the PFR values for metals and dielectrics. For the electromagnetic visible spectrum from $400 \mathrm{~nm}$ to $700 \mathrm{~nm}$, it has been reported that the PFR for metals usually remains below 2 for most specular angle of incidence [4.5].

For varying transmission axis of the external linear polarizer, the pixel outputs averaged over 30 frames at the $0^{\circ}$ and $90^{\circ}$ polarization sensitive pixels are stored. The transmittance is computed by normalizing the output at $0^{\circ}$ and $90^{\circ}$ by the intensity at the intensity sensitive pixel in polarization sense region 1 and 2. The $P F R$ is then calculated from the maximum $I_{\max }(x, y)$ and minimum transmittances $I_{\min }(x, y)$ determined using the same measurement setup shown in figure 4.5 and equation (4.27).

The experimentally obtained PFRs for aluminum and plastic in the polarization sense regions 1 and 2 are shown in figure 4.13 . 


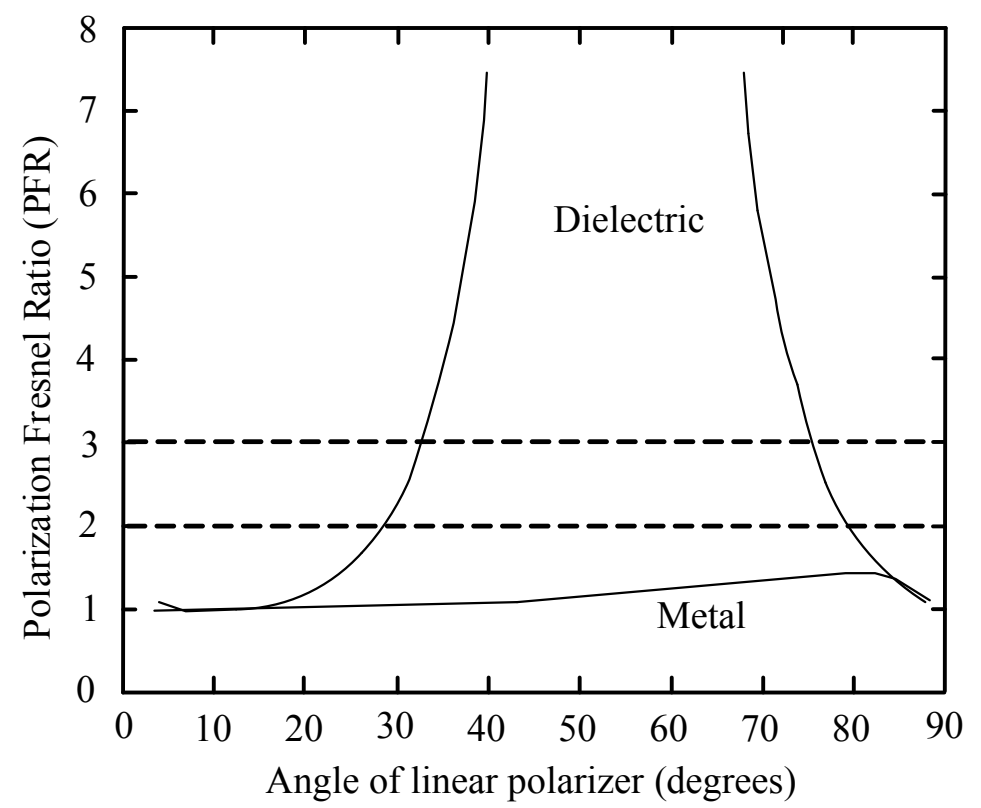

Figure 4.12: Theoretical polarization Fresnel ratio [4.5].

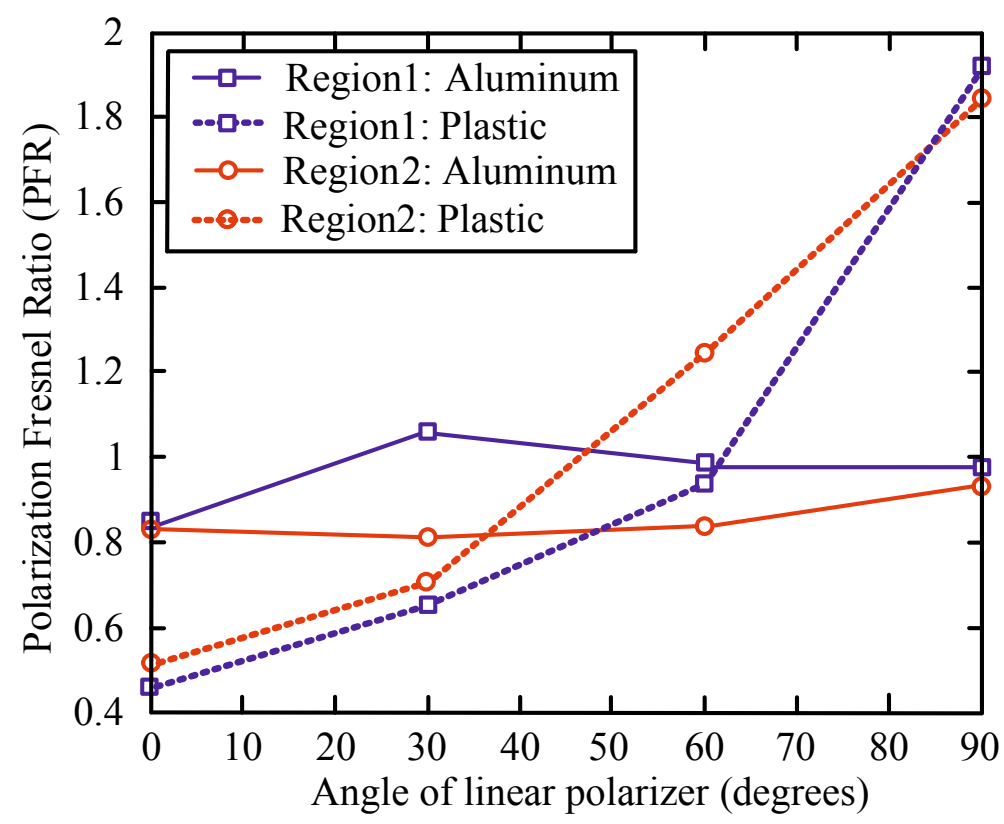

Figure 4.13: Polarization Fresnel ratio in sense region 1 and 2. 
The $P F R$ for aluminum is found to be in the range of 0.8 to 1 for all specular angles of incidence while the $P F R$ for dielectrics rapidly increases near the Brewster angle. Theoretically for a specular angle of incidence greater than the Brewster angle, the $P F R$ for aluminum is always smaller than 2 [4.5]. A $P F R$ value of nearly 2 can be considered to be a dielectric. The PFR computations using the polarization sense regions 1 and 2 offer a good match to the theoretical studies and thus can be used to classify the materials into metals and dielectrics.

\subsection{Metal classification}

The Fresnel reflection theory can also be used to classify among conductive metallic surfaces. The Fresnel reflection coefficients depend on the index of refraction $\epsilon$ and the specular angle of incidence $\varphi$ as shown in equations (4.17).

The basic equations for the propagation of a plane wave in a conducting medium differ from those relating to propagation in a transparent dielectric medium only in that the real constants are replaced by complex constants. The complex index of refraction $\epsilon$ is as denoted by equation (4.34).

$$
\epsilon=n-i \kappa
$$

where $n$ is the simple index of refraction while $\kappa$ is called coefficient of extinction. $\kappa$ is a measure of how well a particular material scatters and absorbs electromagnetic waves. A material with low $\kappa$ allows for easy transmission of the electromagnetic waves and vice versa. The index of refraction for dielectrics is a real number as the coefficient of extinction is negligible, while the index of refraction for metals is a complex number. The components of the index of refraction $n$ and $\kappa$ are related to electromagnetic physical parameters of a material surface [4.12] as

$$
\begin{aligned}
& n^{2}=\frac{v \gamma c^{2}}{2}\left[1+\sqrt{1+\left(\frac{\lambda \sigma}{2 \pi c \gamma}\right)^{2}}\right] \\
& \kappa^{2}=\frac{v \gamma c^{2}}{2}\left[-1+\sqrt{1+\left(\frac{\lambda \sigma}{2 \pi c \gamma}\right)^{2}}\right]
\end{aligned}
$$

where $\sigma$ is the conductivity of the material surface, $v$ is the electrical permittivity, $\gamma$ is the magnetic permeability, $\lambda$ is the incident wavelength of light and $c$ is the 
speed of light in vacuum respectively.

The reflection and absorption of light by metals is also influenced by the skin effect [4.13]. It influences the optical behavior of noble and other metals with high electrical conductivity (such as $\mathrm{Cu}, \mathrm{Al}$ ). A perfect conductor is characterized by infinitely large conductivity. However this means that a perfect conductor would reflect the entire incident light and it would not allow the penetration of the electromagnetic wave to any depth. Thus the reflection coefficients will be high for highly conductive materials while it will be low for lowly conductive materials. To observe the different reflection behavior for different metals of varying conductivity, the measurement setup of figure 4.5 was used. The linear polarization filter was removed and unpolarized light from the DC source was incident on the metal surface. All measurements were done for a light wavelength of 550nm. The reflected light intensity reaching the image sensor was measured using a light meter. Figure 4.14 shows the measured reflected intensity from metal surfaces of varying conductivity for the polarization sense regions 1 and 2 respectively.

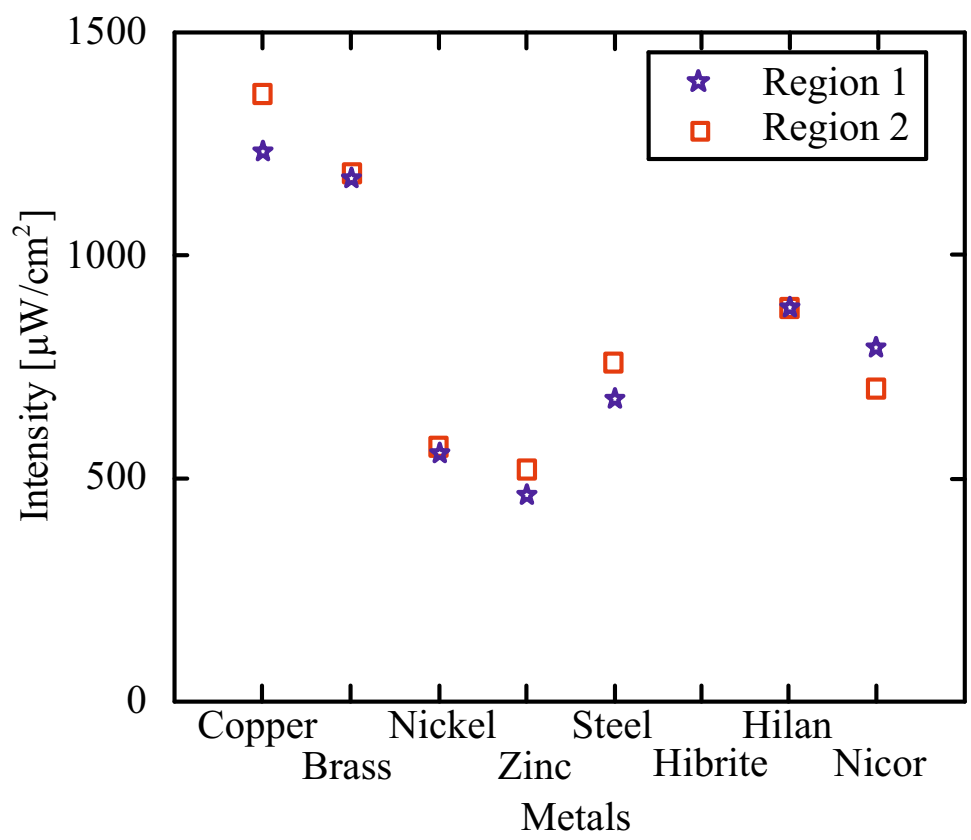

Figure 4.14: Reflected intensity from various conducting metals in sense region 1 and 2.

It is observed that copper and brass have a higher conductivity than the 


\begin{tabular}{|c|c|c|}
\hline \multirow{2}{*}{ Metals } & \multicolumn{2}{|c|}{ Intensity $\left[\mu \mathrm{W} / \mathrm{cm}^{2}\right]$} \\
\cline { 2 - 3 } & Region 1 & Region 2 \\
\hline Copper & 1360 & 1230 \\
\hline Brass & 1184 & 1170 \\
\hline Nickel & 575 & 557 \\
\hline Zinc & 516 & 465 \\
\hline Steel & 758 & 678 \\
\hline Nicor & 703 & 791 \\
\hline Hibrite & 796 & 691 \\
\hline Hilan & 883 & 880 \\
\hline
\end{tabular}

Table 4.3: Reflected intensity from various conducting metals in sense region 1 and 2 .

other metals used (nickel, zinc, steel, hilan, nicor and hibrite) and thus show a higher reflected intensity. This agrees with our previous discussion that higher conductive surfaces reflect the entire incident light due to skin effects. Hilan, nicor and hibrite, which are forms of steel, show relatively the same reflected intensity range. The conductivities of nickel and zinc are higher than that of steel, however the reflected intensity is lower. This could be due to the variations in the electrical and magnetic permeability for a given specular angle of incidence.

\subsubsection{Metal classification using PFR}

From equations (4.35) and (4.36) it can be inferred that, with all other physical parameters remaining constant, the index of refraction increases with an increase in the conductivity of the material. Thus it can be said that the Fresnel equations depend on the conductivity of the material surface. It was further observed in section 4.3.4 that as the conductivity increased from dielectrics to metals, the Fresnel reflection coefficients increased, reducing the $P F R$ as a function of the specular angle of incidence. The variation in the conductivity among various metals will affect the Fresnel reflection coefficients which will further vary the PFR. Changes in the physical parameters in equations ( (4.35) and (4.36) for various metal surfaces would result in different reflection behavior.

Table 4.4 shows the theoretical and experimentally obtained $P F R$ for copper, zinc and aluminum in both polarization sense regions 1 and 2 . The measurement set up is the same as the one shown in figure 4.5.

The theoretical values are the maximum values where the $P F R$ were averaged 


\begin{tabular}{|c|c|c|c|c|}
\hline \multirow{2}{*}{ Metals } & Conductivity & Max. & \multicolumn{2}{|c|}{ Experimental PFR } \\
\cline { 4 - 5 } & $(/ \mathrm{cm} \Omega)$ & theoretical PFR & Region 1 & Region 2 \\
\hline Copper & $0.596 \times 10^{6}$ & 1.33 & 1.12 & 1.08 \\
\hline Alum. & $0.3776 \times 10^{6}$ & 1.07 & 1.25 & 1.15 \\
\hline Zinc & $0.166 \times 10^{6}$ & 1.31 & 1.21 & 1.28 \\
\hline
\end{tabular}

Table 4.4: Theoretical and experimental PFR values.

over different index of refraction corresponding to wavelengths in the visible spectrum [4.5]. The PFR for copper was found to be lower than that of Zinc and Aluminum in both polarization sense regions 1 and 2 .

Among the selected three metallic surfaces, the conductivity of copper is the highest, and aluminum is more conductive than zinc. The higher conductivity of copper produces higher Fresnel reflection coefficients which in turns results in lower $P F R$, seen in the experimentally obtained $P F R$ in both polarization sense region 1 and 2. Zinc, being the least conductive, should have higher $P F R$, as seen in the measurements of polarization sense region 2. In polarization sense region 1 zinc shows lower PFR than aluminum. This could be the result of the variations in the specular angle of incidence of the light source, as the specular angle of incidence of the light source was not well controlled. However a clear distinction between the degrees of polarization for copper, zinc and aluminum is visible, and the $P F R$ values are lower than 2 as stated in [4.5].

To extend the study of changes in the $P F R$ with conductivity, more metallic surfaces were selected and the experimentally obtained $P F R$ are shown in the figure 4.15.

The lower conducting materials steel, hibrite, hilan and nicor show higher PFR values in both the polarization sense regions 1 and 2 , while highly conductive metals show lower $P F R$. The high $P F R$ for the low conducting materials is due to reduced reflection and lower Fresnel reflection coefficients. A clear distinction can be observed among the PFR values of various test metal surfaces of varying conductivity.

\subsubsection{Metal classification using degree of polarization}

The $D O P$ of highly conductive materials is lower than the $D O P$ of low conducting materials. The partial polarization or $D O P$ introduced in section 4.3.3 was also calculated for various metallic surfaces and the obtained $D O P$ in the polarization sense regions 1 and 2 is shown in figure 4.16. The resultant plots 


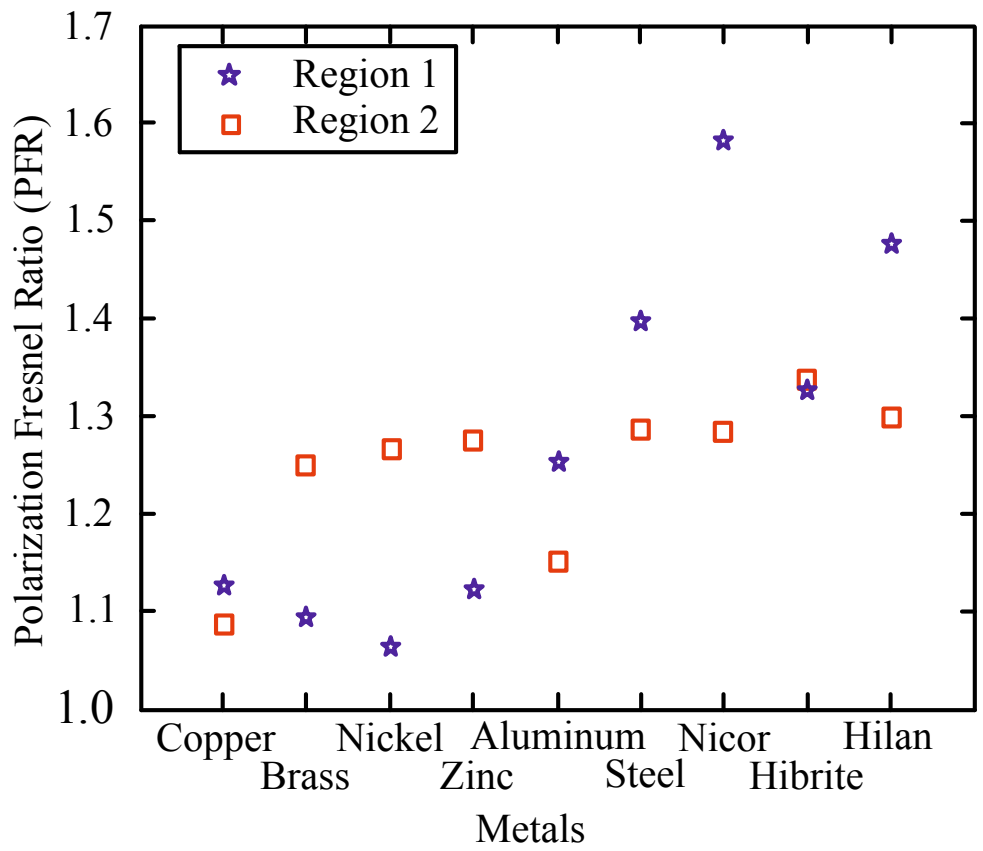

Figure 4.15: Measured PFR for various conducting metals in sense region 1 and 2.

\begin{tabular}{|c|c|c|}
\hline \multirow{2}{*}{ Metals } & \multicolumn{2}{|c|}{ Experimental DOP } \\
\cline { 2 - 3 } & Region 1 & Region 2 \\
\hline Copper & 0.059 & 0.040 \\
\hline Brass & 0.044 & 0.111 \\
\hline Nickel & 0.030 & 0.117 \\
\hline Zinc & 0.057 & 0.121 \\
\hline Alum. & 0.112 & 0.070 \\
\hline Steel & 0.165 & 0.125 \\
\hline Nicor & 0.140 & 0.144 \\
\hline Hibrite & 0.192 & 0.129 \\
\hline Hilan & 0.226 & 0.124 \\
\hline
\end{tabular}

Table 4.5: Measured PFR for various conducting metals in sense region 1 and 2.

show similar behavior as the $P F R$ plots.

It is observed that the $D O P$ of metal surfaces varies between 0 and 0.25 in both the polarization sense regions 1 and 2 . The lower conducting metal surfaces, such 
as steel and its varieties occupy the higher band of the range while the lower band is occupied by highly conducting metal surfaces.

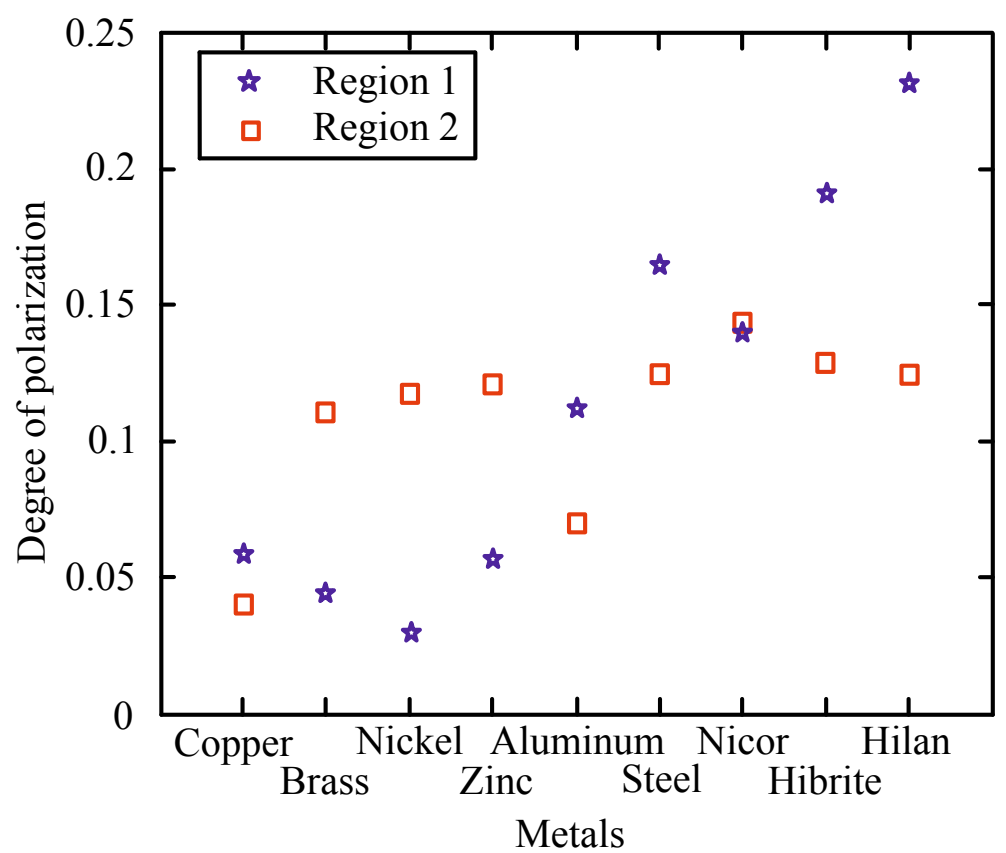

Figure 4.16: Measured degree of polarization in sense region 1 and 2.

\begin{tabular}{|c|c|c|}
\hline \multirow{2}{*}{ Metals } & \multicolumn{2}{|c|}{ Experimental DOP } \\
\cline { 2 - 3 } & Region 1 & Region 2 \\
\hline Copper & 0.059 & 0.040 \\
\hline Brass & 0.044 & 0.111 \\
\hline Nickel & 0.030 & 0.117 \\
\hline Zinc & 0.057 & 0.121 \\
\hline Alum. & 0.112 & 0.070 \\
\hline Steel & 0.165 & 0.125 \\
\hline Nicor & 0.140 & 0.144 \\
\hline Hibrite & 0.192 & 0.129 \\
\hline Hilan & 0.226 & 0.124 \\
\hline
\end{tabular}

Table 4.6: Measured degree of polarization in sense region 1 and 2.

There is however a difference in behavior in the two polarization sense regions 
for both the PFR and DOP measurements, as seen in figures 4.15 and 4.16. In region 2, the difference between the copper and other conducting materials is more pronounced than in region 1 . This can be explained by considering the presence of an additional $45^{\circ}$ linear polarizer in region 2 along with the fact that the Fresnel coefficients measured are truly a regional average. For highly reflective surfaces the response of the $45^{\circ}$ sensitive pixels increases the Fresnel coefficients average, thus further decreasing the $P F R$ and DOP. For copper $R_{s}(x, y) \approx R_{p}(x, y)$, while for other conducting materials the average $R_{s}(x, y)$ increases due to the $45^{\circ}$ polarizer response compared to $R_{p}(x, y)$, thus increasing the $P F R$ and DOP. For very low conducting surfaces the $45^{\circ}$ sensitive pixels do not affect the Fresnel coefficient measurements thus the measured average $R_{s}(x, y)$ decreases reducing the $P F R$ and $D O P$.

\subsection{Conclusions}

The state of polarization for the diffuse and specular components of the reflection depend on the reflecting surface, and the measurement of the state of polarization of the reflected light serves as an indicator for the type of material surface. The magnitude of oscillations of the maximum and minimum transmitted irradiance due to the variation in the reflection pattern of metal and dielectric surface was found to be useful in classifying them.

Three measurement metrics namely, the degree of polarization, Stokes degree of polarization and polarization Fresnel ratio are described in this chapter and are shown to vary with the variations in the reflection pattern of the metal and dielectric surface. These measurement metrics are thus useful in classifying a metal surface from a dielectric one.

Among the three measurement metrics the polarization Fresnel ratio is the simplest to calculate. The ratio of the irradiance measured at $90^{\circ}$ and $0^{\circ}$ polarization sensitive pixels can be obtained using an analog divider which then relates to the $P F R$. Furthermore the $P F R$ for metals does not vary much with the variations in the linear polarizer angle and thus metals are easily distinguishable from dielectrics which show marked variance.

For the implementation of the degree of polarization and Stokes degree of polarization additional subtraction and addition functionalities are required. Both these functionalities can be achieved using the amplifier in the pixel, however they are more complex, compared to the PFR measurements. The polarization of the reflected component of the light wave varies with the conductivity of the metallic surface and this was further explored and shown to be able to serve as a 
tool to classify among highly conductive and lowly conductive metallic surfaces.

\subsection{References}

[4.1] G. Klinker, S. Shafer, and T. Kanade, "Using a color reflection model to separate highlights from object color," Proceedings of International Conference on Computer Vision, pp. 145-150, 1987.

[4.2] S. Shafer, "Using color to separate reflection components," Color Research Applications, vol. 10, no. 4, pp. 210-218, 1985.

[4.3] G. Healey and W. Blanz, "Identifying metal surfaces in color images," Proceedings of Conference in Optics, Electro-Optics, and Sensors, 1988.

[4.4] G. Healey and T. Binford, "Predicting material classes," Proceedings of DARPA Image Understanding Workshop, pp. 1140-1146, 1988.

[4.5] L. Wolff, "Polarization based material classification from specular reflection," IEEE Transactions on Pattern Analysis and Machine Intelligence, vol. 12, no. 11, pp. 1059-1071, 1990.

[4.6] H. Chen and L. Wolff, "Polarization phase based method for material classification and object recognition in computer vision," International Journal of Computer Vision, vol. 28, no. 1, pp. 45-56, 1999.

[4.7] S. Tominaga and A. Kimachi, "Polarization imaging for material classification," Optical Engineering, vol. 47, no. 12, pp. 123201-1-14, 2008.

[4.8] P. Drude, The theory of optics, p. 278. New York: Dover publications inc., 1959. ISBN: 486605329.

[4.9] M. Sarkar, D. S. Segundo, C. van Hoof, and A. Theuwissen, "Integrated polarization analyzing CMOS image sensor," Proceedings of IEEE International Symposium on Circuits and Systems, pp. 621-624, 2010.

[4.10] D. Brewster, "On the laws which regulate the polarization of light by reflection from transparent bodies," hilosophical Transactions of the Royal Society of London, vol. 105, p. 128, 1815.

[4.11] V. Gruev, J. V. der Spiegel, and N. Engheta, "Advances in integrated polarization imaging sensors," IEEE Life Science Systems and Applications Workshop, pp. 62-65, 2009.

[4.12] R. Siegal and J. Howell, Thermal radiation heat transfer. New York: McGraw-hill, 1981. ISBN: 1560328398. 
[4.13] P. Gilberd, "The anomalous skin effect and the optical properties of metals," Journal of Physics F: Metal Physics, vol. 12, pp. 1845-1860, 1982. 


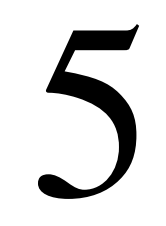

\section{Navigation using CMOS polarization sensor}

The navigational strategies of insects using skylight polarization are interesting for applications in autonomous agent navigation because they rely on very little information for navigation. The skylight polarization pattern for navigation varies in a systematic fashion both in plane (e-vector) and degree of polarization, depending only on the direction of the observation point relative to the angular position of the sun. This is found to be very efficient and reliable for real time navigation.

In this chapter, a polarization navigation sensor using the Stokes parameters to determine the orientation and position is presented. Section 5.1 discusses the two most common navigation algorithms prevalent in most animals: egocentric and geocentric. The working principle of the implemented algorithm is based on egocentric navigation, predominant in insects. The celestial compass based on skylight polarization is presented in section 5.2. Section 5.3 discusses the navigation compass employed by insects and in section 5.4 some of the implemented models for autonomous agent navigation based on the insect's model are presented. In section 5.5 the proposed polarization based compass is presented. The variation in the degree of polarization with changes in the polarized light can be used as a compass and conversely from the degree of polarization the incoming light ray direction can be determined. The computation of ellipticity and azimuthal angles allows for on-chip position detection based on the angle of the incoming light ray with little complexity. This can further be used as a sun position detector based on the skylight polarization. The ability to compute on-chip or in real-time the positional information would result in highly miniaturized navigational sensors. 


\subsection{Introduction}

There are two types of spatial representation created by navigation. One type is a cognitive map or survey representation of space and the other is a route representation [5.1], [5.2]. Cognitive mapping or survey representation of the space defines the Euclidean relations (straight line distance and direction) among relevant landmarks within a coordinate reference system centered on the environment. Route representation or spatial representation of navigation relies on learning the points in the route.

The role of vision in navigation and cognitive mapping has been extensively studied [5.3]. Based on the cognitive mapping, navigation can be broadly classified into geocentric and egocentric [5.3], [5.4]. Humans navigate using geocentric form of navigation. In geocentric navigation, one uses its cognitive map and its orientation with respect to geocentric coordinates in order to set a course towards a goal. In this case visual cues such as landmarks become very important to generate the cognitive maps. In the absence of information about the location of nearby objects in the environment, humans have difficulty monitoring their travel trajectories, even for short paths. Egocentric navigation, is known to be used by some insect species such as the desert ant (Cataglyphis fortis) which relies on path integration, where the movement cues of the navigator are continuously integrated [5.4]. Animals using egocentric navigation are able to track distance and direction in order to estimate their position even in the absence of visual landmarks.

The progress in the field of autonomous agent navigation has been slower than expected, especially after the initial excitement and rapid advances in the early days of the research [5.5]. It is interesting to consider why autonomous navigation is so difficult. A survey into the available algorithms for autonomous agent navigation reveals that most of them are written to make them understandable for the human operator, mostly employing Cartesian coordinates $(x, y, z)$ to represent the location of the feature [5.6]. Current sensors employed for navigation applications, and especially robotic navigations, use a generalized algorithm of capturing two-dimension images using a standard CMOS or CCD camera and then processing those captured image sequences for vector calculations to determine the motion vector and the direction vector with reference to the intimal position. The use of image sequences to determine motion and direction vectors is more related to the geocentric form of navigation, where the emphasis is more on the process of creation of two-dimension or three-dimension maps to guide the autonomous agents. This has the advantage of offering more control over 
the autonomous agent but demands large computational resources and lacks the robustness required for real-time applications. Thus these methods may not be the best solution for artificial autonomous navigation agents.

Insects, on the other hand are able to solve complex navigation problems in real-time relying on egocentric forms of navigation. Thus egocentric forms of navigation is best suited for autonomous agents than the geocentric form. This ability of insects to navigate effortlessly in complex environments without stressing their nervous system has already been a subject of research in robotics, and the cheap computational strategies that these insects use to navigate have been modeled by various researchers [5.7], [5.8], [5.9].

Path integration is the basis of vector navigation in egocentric forms of navigation [5.10], and it requires knowledge of the direction of travel and the distance travelled. In this thesis, the focus is on determining the direction of travel and not on distance measurements. To determine the direction of travel a reference direction is needed. The direction is then always expressed relative to the reference. In conventional autonomous agent navigation algorithms, multiple images captured by a conventional camera and complex image processing algorithms are used to determine the angle of travel [5.6], [5.11]. The implementation of these algorithms needs very complex digital logic, and the image processing requires high power consumption and high bus bandwidth [5.12]. This limits the design of miniature and low-power vision-based navigation systems.

Insects such as the Saharan desert ant (Cataglyphis fortis), use the position of the sun to determine their direction of travel [5.13]. Since the movement of the sun is constant and equal to approximately $1^{\circ}$ every 4 minutes, it serves as a very good directional reference. To determine the direction of travel, ants use the celestial compass either based on the direct sunlight (sun compass) or on the pattern of the polarized skylight (polarization compass) [5.14]. Santschi [5.15] was the first to show that ants can use the sun as a compass cue. Genera of ants like Messor and Monomorium uses sun compass. In Cataglyphis fortis the polarization compass dominates over sun compass [5.14].

To determine the position of the sun using direct sunlight, conventional analog sun position sensors can be used. These sensors measure the position of the sun by allowing the light from the sun to pass through a pin-hole array and illuminate a certain region of the imaging array [5.16] as shown in figure 5.1.

The position of the illuminated region is then used to compute the altitude and position of the sun with respect to the sensor. State of the art digital sun sensors such as [5.17] use the centroid method to compute the angular position of the sun while row profiling is used in the winner takes all (WTA) method proposed 


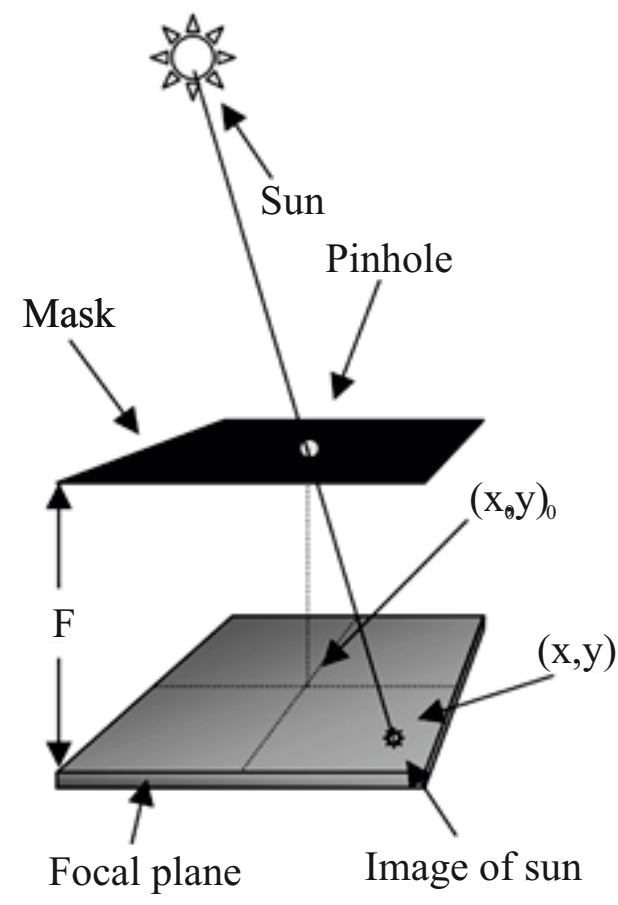

Figure 5.1: Sun position detection model using image centroid.

by [5.18]. The prerequisite of these sun sensors is that they need to see the sun, which is not always possible, for example in a cloudy day. Additionally, these sensors need an additional pin-hole array and digital processors to compute the centroid of the obtained image.

The other compass used by insects is the polarization compass. A polarized skylight navigation system uses the extensive pattern of polarized skylight generated by the scattering of the light rays on collision with the air molecules. Each partially polarized skylight ray exhibits a predominant vibration direction (e-vector) perpendicular to the plane of the scattering angle. The polarization pattern varies in a systematic fashion both in plane (e-vector) and degree of polarization, according to the position of the sun. These polarization patterns in the sky are used by ants as a reference for compass orientation. This makes their navigation pattern completely independent of external visual cues. The celestial compass based on the skylight polarization is described in section 5.2. 


\subsection{Celestial compass based on skylight polariza- tion}

Direct sunlight is unpolarized. When this unpolarized light enters the earth's atmosphere, it collides with the air molecules or is scattered because of the fluctuations in the air density. The scattering particles, the air molecules, are much smaller than the wavelength of the light striking them, thus the scattered intensity is the same in the forward and the backward directions. This scattering is often explained using the Rayleigh or molecular scattering theory, proposed in 1871 by Lord Rayleigh. The scattering caused by the randomly located air molecules is considered incoherent and thus can be assumed to be coming from only one particle. This scattering is dependent on the wavelength of the incoming light, where intensity of scattering is inversely proportional to the fourth power of the wavelength [5.19]. Blue light with wavelength at around $425 \mathrm{~nm}$ scatters 5.5 times more energy than red light of wavelength $650 \mathrm{~nm}$. The higher scattering of the blue light explains the blue appearance of the sky.

The scattered light by the air molecules, or skylight, is partially polarized which means it is a composition of both the unpolarized natural light and also a linearly polarized component [5.20]. Circular and elliptical polarizations, explained in chapter 3, do not usually occur in the sky and thus only the linear polarization component is of interest. The scattered light has an $e$-vector oriented perpendicular to the plane of the scattering, i.e., perpendicular to the great circle passing through the sun and the point observed. Consequently, the $e$-vectors in the sky form concentric circles around the sky. This skylight polarization is mainly dependent on the angle between the viewing direction and the sun and the clearness of the sky in the viewing direction which affects the degree of polarization. There is an indirect relationship between the degree of polarization and the skylight intensity: for sun positions near the zenith, the degree of polarization is quite high while at the horizon the degree of polarization is weaker [5.21].

The degree of linear polarization is not constant over the entire sky but depends on the solar position of the sun as well as the atmospheric conditions. The strongest linear degree of polarization is observed during the sunrise and sunset at $90^{\circ}$ from the sun position. The deeper and clearer the blue of the sky is, the stronger is the degree of polarization. However a total degree of polarization is never observed even when the viewing angle is $90^{\circ}$ because secondary scattering and diffuse reflections always reduce the degree of polarization. Clear blue skies are strongly polarized while hazy skies exhibit very little polarization. 
In clear sky, the polarization patterns are quite regular and depend very strongly on the position of the sun. The position of the sun in the sky relative to an observer on earth is defined by its solar elevation angle and its solar azimuth angle. The elevation angle is the angle between the line to the center of the sun and the horizontal plane. Horizontal plane is the reference plane for the solar elevation. when the sun is on the horizon, the elevation is $0^{\circ}$ while when directly overhead the elevation angle is $90^{\circ}$. The azimuth angle is the angle between true south and the point on the horizon directly below the sun, the reference plane for the solar azimuth is the vertical plane running north-south through the poles. Since the $e$-vector orientation depends only on the plane of the scattering angle, the $e$-vector orientation is nearly independent of the atmospheric disturbance factors. The celestial $e$-vector pattern for two different elevations of the sun is shown in the figure 5.2.

The sun moves along its arc with uniform angular velocity of 15 degrees per hour, while the solar azimuth does not. Its angular velocity depends on the time of day, time of year and geographical latitude. Figure 5.2 shows two distinctive features. First, the gradient of the $e$-vector is related to the position of the sun and second, the pattern of polarization has mirror symmetry with respect to the plane defined by the solar meridian (SM) and the anti-solar meridian (ASM). The solar meridian is the line between the sun and the zenith (highest point in the sky). The solar meridian rotates around the zenith as the position of the sun changes. Due to the westward movement of the sun, the $e$-vector pattern rotates around its zenith but retains the two important characteristics over the day, the mirror symmetry and that along the symmetry line the $e$-vectors are always perpendicular to the solar meridian [5.7].

A model to understand the polarization of the skylight is shown in figure 5.3. The transmission of light from the sun $(S)$ to the scatterer $(P)$ and the observer $(M)$ is shown in the figure. The angle of elevation for the scatterer and the observer are $\psi_{s}$ and $\psi_{p}$ respectively.

The parallel and the perpendicular component of the polarized light radiates differently. The perpendicular component radiates omni-directional while the parallel component radiates proportional to $\cos \left(\psi_{p}-\psi_{s}\right)$ in figure 5.3, the difference of these two components produces the partial polarization of the skylight. The observer views the skylight through the linear polarizer with transmission axis at the angle $\zeta$ to the normal of the principal plane ( $x$-axis). As the observer rotates the polarizer, he measures the maximum and minimum in 


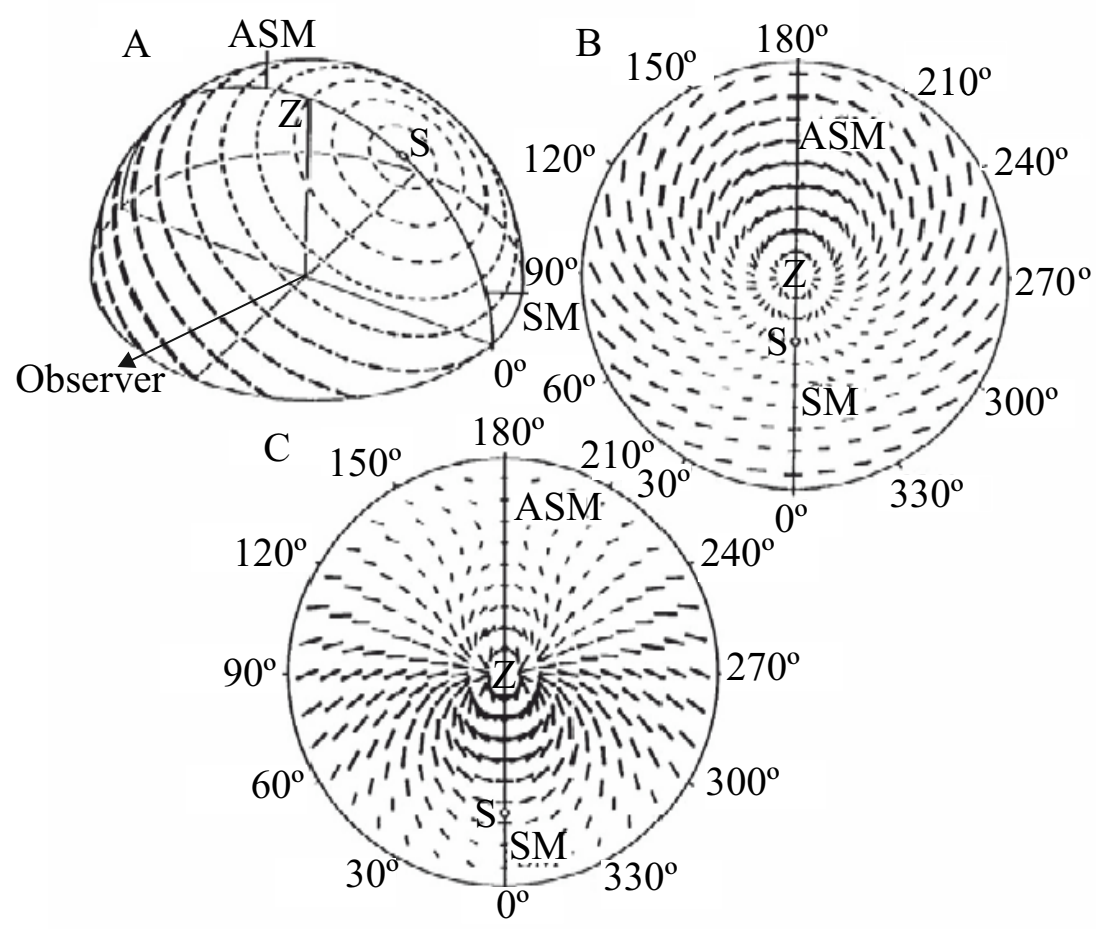

Figure 5.2: Celestial e-vector pattern (A) Three-dimensional representation of the e-vector pattern, the orientation, length and width of each black bar corresponds to e-vector direction and the degree of polarization respectively.

The symmetery line running through sun $(S)$ and zenith $(Z)$, is called "solar meridian" (SM) on the side of the sun and "anti-solar meridian" $(A S M)$ on the opposite side. (B) Two-dimensional for sun elevation at $60^{\circ}$ and $(C)$

Two-dimensional for sun elevation at $24^{\circ}$.

the irradiance. This can be used to compute the degree of polarization [5.21] as:

$$
D O P=\frac{I_{\max }-I_{\min }}{I_{\max }+I_{\min }}=\frac{\sin ^{2}\left(\psi_{p}-\psi_{s}\right)}{1+\cos ^{2}\left(\psi_{p}-\psi_{s}\right)}
$$

where $D O P$ is the degree of polarization, $I_{\max }$ and $I_{\min }$ are the maximum and minimum transmitted intensity, $\psi_{s}$ and $\psi_{p}$ are the angle of the sun and the scatterer respectively. This scattered sunlight is partially polarized depending on the scattering angle, the angle between the incoming direct solar light rays and the outgoing skylight rays. The degree of partially linear polarized skylight 


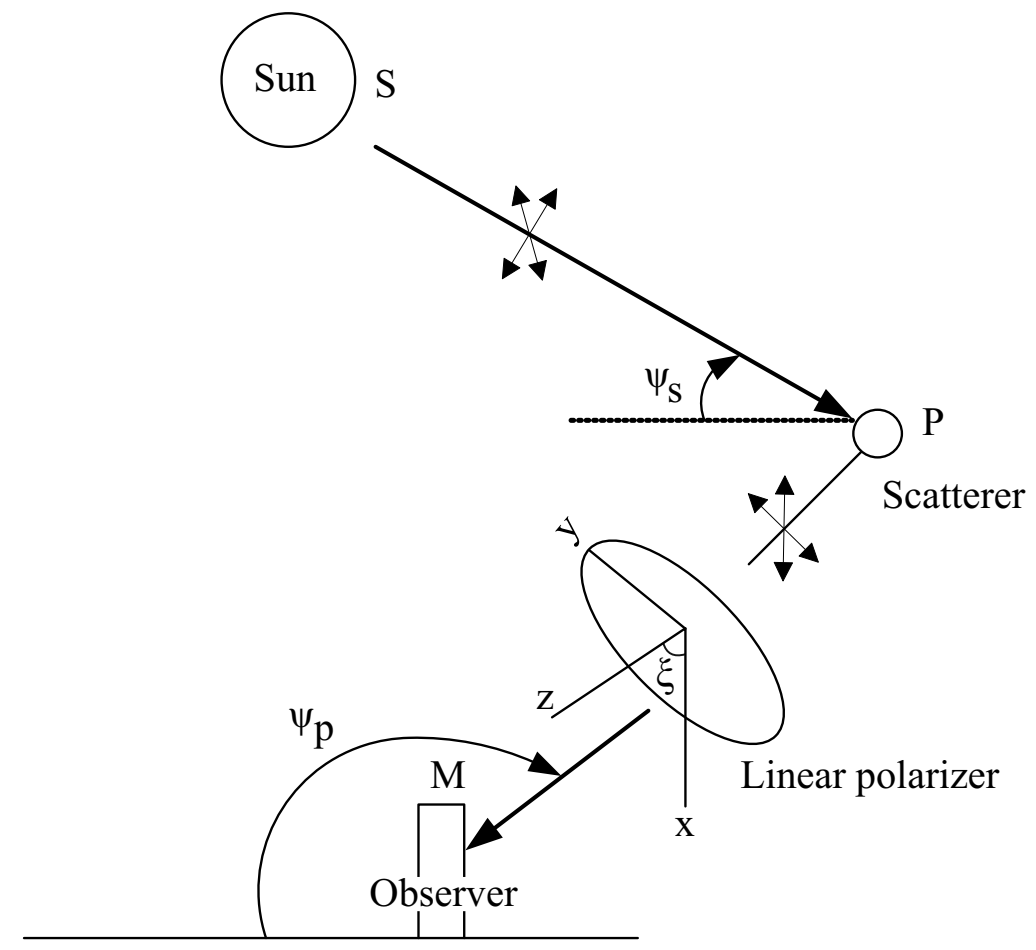

Figure 5.3: Model to observe the skylight polarization.

can be expressed in terms of the scattering angle $\theta$ where $\theta=\psi_{p}-\psi_{s}$.

The degree of polarization as seen from equation (5.1) only depends on the difference in the angles $\psi_{p}-\psi_{s}$. Thus in the direction of the sun, when the scattering angle $\theta=0^{\circ}\left(\psi_{p}=\psi_{s}\right)$, the skylight is completely unpolarized $(D O P=0)$. The maximum degree of polarization $(D O P=1)$ would occur for a scattering angle of $90^{\circ}\left(\psi_{p}=\psi_{s}+\pi / 2\right)$.

In figure 5.4, the theoretical degree of linear polarization predicted by equation (5.1) for varying sun elevation angle is plotted along with the measurement results found in literature. For the measurements the observer is viewing the the zenith sky $\psi_{p}=\pi / 2$ as the sun rises ( $\psi_{s}$ increases) in figure 5.3. The two experiments conducted on Mauna Lao in Hawaii [5.20] and Bocaiuva in Brazil [5.22] both on high altitudes on a clear day, shows that the measured degree of polarization truly changes with the position of the elevation angle of the sun. 


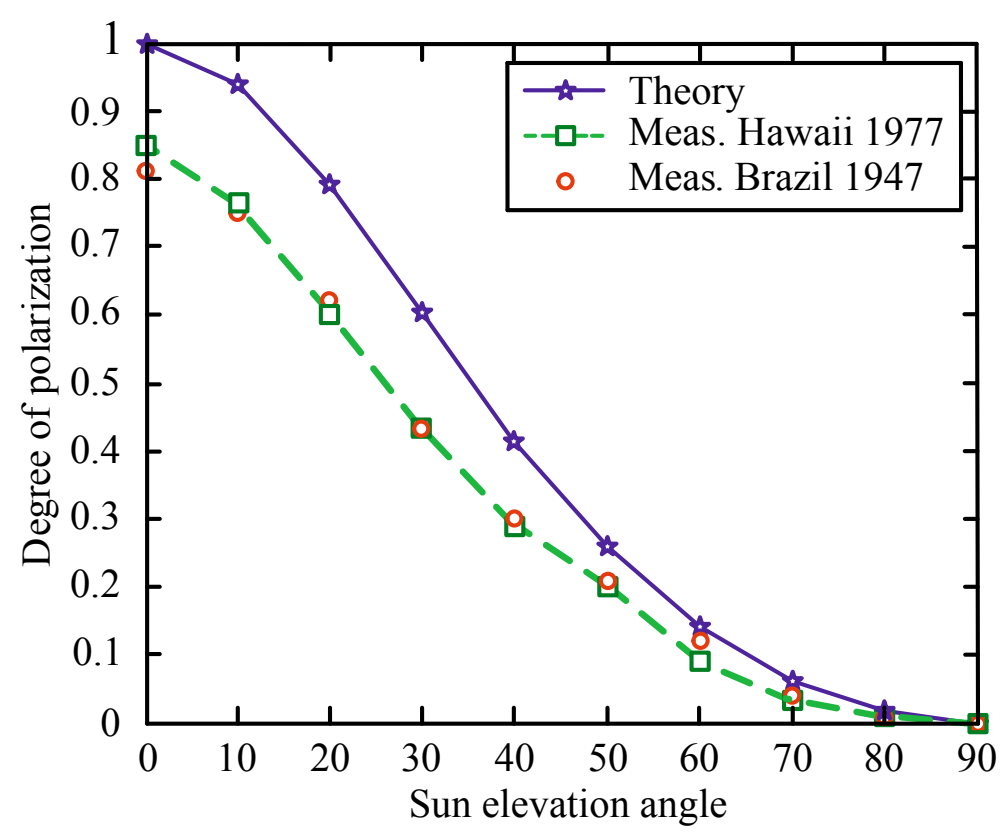

Figure 5.4: Variation in degree of polarization with changes in the sun elevation angle.

\subsection{Navigation using polarized light by insects}

Deserts are barren lands with almost no rain and vegetation. Navigation in such an environment is a challenge due to the absence of any visual landmarks. Humans who rely mostly on geocentric navigation depending on the visual cues and landmarks to form the cognitive map find it difficult to navigate in a desert even with a complex evolved brain. One habitant of such an environment is the Cataglyphis fortis (Insecta: Hymenoptera: Formincidae) shown in figure 5.5. For foraging activities such as search of food these ants travel up to twenty thousand times their own body length and go back home in a determined manner straightly without getting lost on the way [5.23]. This is truly amazing considering the absence of any visual cues or landmarks.

Usually when foraging for food ants such as wood ants would mark the route of their travel by placing olfactory marks [5.24]. This serves two purposes, one to let other ants know the direction towards food and also helps in serving as a return path. This is not possible with Cataglyphis fortis as the sand in the desert is not cohesive and would be carried away easily by wind. Also the dry environment and the hot surface temperature will evaporate the pheromones too quickly and 


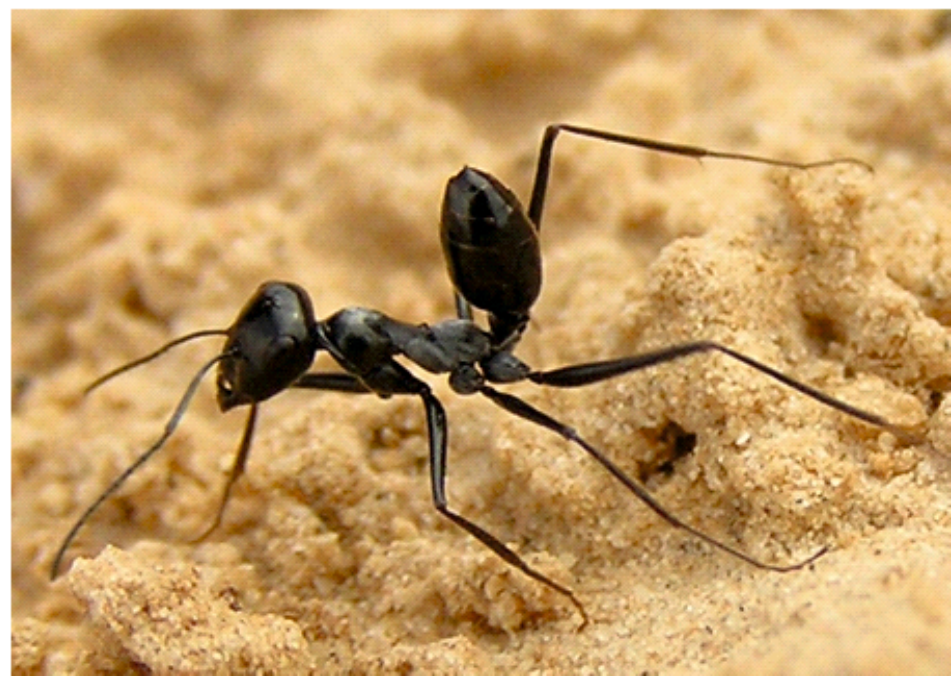

Figure 5.5: Desert ant (Cataglyphis fortis).

thus cannot be used for stable marking. The navigation pattern of Cataglyphis fortis has perplexed biologists for a long time and theories about its navigational pattern have been postulated since 1904 when Pieron [5.25] argued that the ants while homing retrace the steps made during its outward journey by employing certain proprioceptive means. This argument was proven wrong by Cornetz in 1910 [5.26]. He proposed that the ants integrate their path rather than retrace the steps. This form of navigation is quite similar to that used by sailors at sea before the invention of global positioning system (GPS). This concept of path integration by Cataglyphis fortis is now a widely accepted view [5.10], [5.27].

The path integration as the basis of vector navigation requires knowledge of the direction of travel and the distance traveled to compute the home vector. At each stage of foraging the ant continuously updates its home vector which points to the nest of the starting point of the travel. The compound eyes of ants are adapted to detect the polarization prevalent in the skylight [5.28]. There are three distinct functional regions in the compound eyes of Cataglyphis fortis: the dorsal rim area $(D R A)$, the dorsal area $(D A)$ and the ventral area $(V A)$ [5.29]. The microvilli of the DRA photoreceptors are aligned in parallel along the entire length of the cell from the distal tip of the rhabdom down to its proximal end and are perpendicular to other microvilli of the other photoreceptor cells [5.30]. These mutually orthogonal photoreceptors in the microvilli helps to obtain polarization antagonism. The polarization-antagonism has two important effects: it enhances 
$e$-vector contrast, which allows the neurons to respond to very low degree of polarization and it makes the system insensitive to the variations of absolute light level. That is, the neurons act as differential polarization detectors.

The $e$-vector patterns in the sky are not visible to humans, but ants use these vector directions as a reference for compass orientation. The $e$-vector information collected by the photoreceptors of the $D R A$ is processed by polarization sensitive neurons in the optic lobe (POL neurons). Although most behavioral studies on the polarization compass are performed in bees and ants, due to the difficulty in recording from ant brains most electrophysiological data are obtained from orthopteran insects like the cricket [5.31]. The ants' $P O L$-neurons exhibit opponency and wide visual fields, like the cricket. The $P O L$-neuron of the cricket is shown in the figure 5.6.
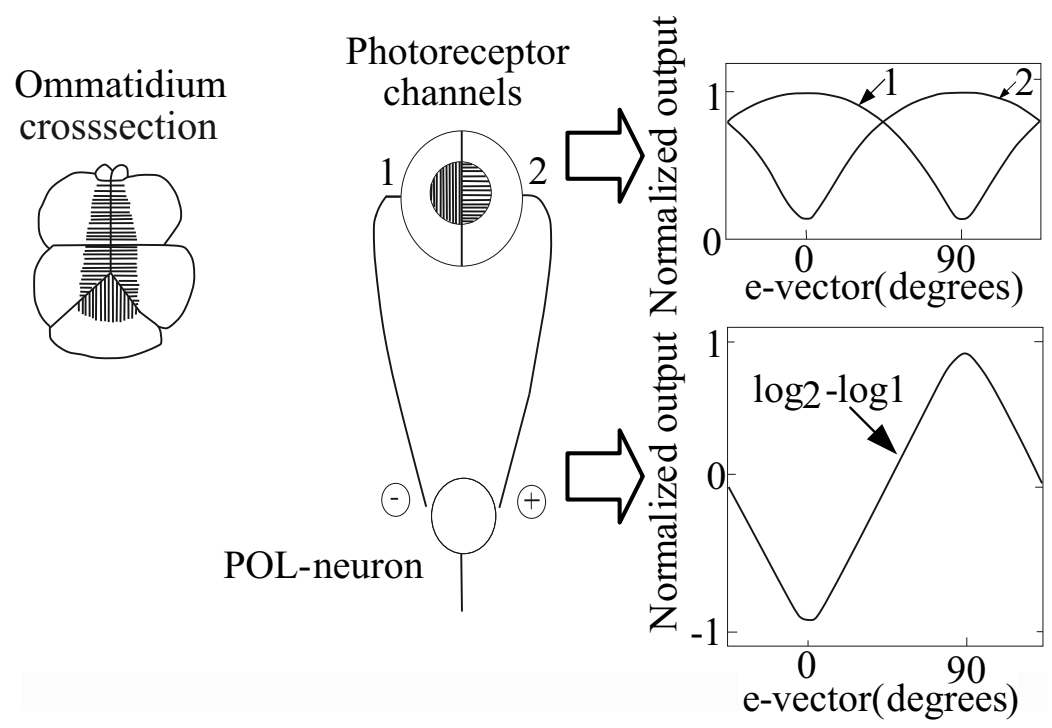

Figure 5.6: (A) Ommatidum cross section of cricket. (B) Principle of operation of polarization opponent neurons (POL-neurons) [5.7].

The $P O L$-neurons receive antagonist inputs from the receptor channels. The response function of the $P O L$-neuron is a sinusoidal function of $e$-vector orientation with an excitatory and an inhibitory part and with the maxima and minima separated by $90^{\circ}$. The response function is the difference of the signals from the receptor channels and in principle determines the $e$-vector within the visual field of the neurons. 


\subsection{Navigation using polarized light for autonomous agents}

The principle of using polarization antagonism prevalent in the compound eye of insects as a polarization based compass for autonomous agents has been successfully modeled by various researchers. Lambrinos et al. [5.7], [5.8] modeled the polarized light sensitive receptors ( $P O L$-neurons) in the Cataglyphis fortis using a pair of photodiodes with linear polarizers on top and a log ratio amplifier as shown in the figure 5.7. The pair of photodiodes with linear polarizers on top receives mutually orthogonal $e$-vector information, mimicking the receptors in the DRA region of the ommatidia of the compound eye of the insects. The response in each of the photodiodes is shifted by $90^{\circ}$. The signal from each diode is then fed to a log amplifier to obtain the logarithmic difference of the intensities received from the two photodiodes, similar in behavior as shown in figure 5.6.
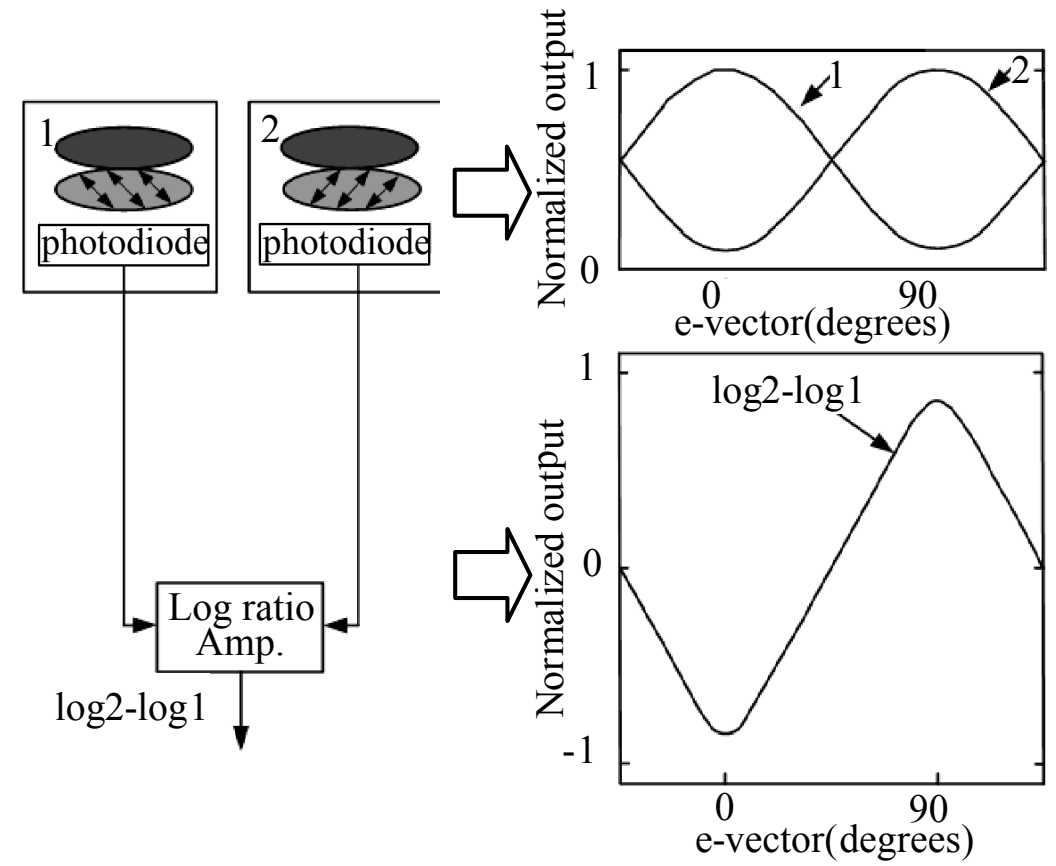

Figure 5.7: Analog implementation of the POL-neuron [5.7].

Lambrinos [5.7] presented an analytical method of determining robot orientation based upon the polarized light neuron receptor geometry of the cricket. The 
three types of $P O L$-neurons in a cricket are tuned to different $e$-vectors oriented approximately $10^{\circ}, 60^{\circ}$ and $130^{\circ}$ relative to the length axis of the head. In the model proposed by Lambrinos et al., sensors with maximum sensitivity at the polarizer axis of $0^{\circ}, 60^{\circ}$ and $120^{\circ}$ were placed on the robot and analytical expressions for orientation were derived based on models of sensor output. The sensor output was described by the following equation:

$$
f(\varphi)=K I[1+d \cos (2 \varphi)]
$$

where $I$ is the total intensity, $I=I_{\max }+I_{\min }, I_{\max }$ is the maximum and $I_{\min }$ is the minimum intensity obtained from the photodiodes with linear polarizer in crossed-analyzer configuration (the two linear polarizers tuned to orthogonal $e$ vectors), $d$ is the degree of polarization, $\varphi$ is the orientation with respect to the solar meridian maximizing $f(\varphi)$, and $K$ is a constant [5.32].

For the three $P O L$ units oriented at of $0^{\circ}, 60^{\circ}$ and $120^{\circ}$ the output of the log amplifier can be expressed as,

$$
\begin{aligned}
& f_{1}(\varphi)=\log \left(\frac{1+d \cos (2 \varphi)}{1-d \cos (2 \varphi)}\right) \\
& f_{2}(\varphi)=\log \left(\frac{1+d \cos \left(2 \varphi-\frac{2 \pi}{3}\right)}{1-d \cos \left(2 \varphi-\frac{2 \pi}{3}\right)}\right) \\
& f_{3}(\varphi)=\log \left(\frac{1+d \cos \left(2 \varphi-\frac{4 \pi}{3}\right)}{1-d \cos \left(2 \varphi-\frac{4 \pi}{3}\right)}\right)
\end{aligned}
$$

where $f_{1}(\varphi), f_{2}(\varphi)$ and $f_{3}(\varphi)$ are the outputs of the $P O L$ units oriented at $0^{\circ}$, $60^{\circ}$ and $120^{\circ}$ respectively.

To derive the compass information from equations (5.3), (5.4) and (5.5) either a scanning model or a simultaneous model [5.7] is used. In the scanning model the first task is to find the solar meridian and use it as a reference direction $\left(0^{\circ}\right)$. The sky is scanned using the system by rotating around its vertical axis to find the maximum. The maximum value of the output signal is obtained when of the polarization filter is aligned to the solar meridian. After finding the reference direction, the information about the current state of the body can be used to find the direction of motion. In the simultaneous model, a look-up table is used to relate the obtained output values to the orientation and direction of motion instead of scanning the sky for a reference. While scanning the sky the maximum output 
obtained is used as a reference.

Equation (5.2) is dependent on the degree of polarization. The degree of polarization changes during the day depending on the position of the sun as shown in figure 5.2. In the scanning model of the compass determination, the degree of polarization is naturally eliminated as only the maximum output value is of interest. In the simultaneous model the degree of polarization has to be continuously updated based on the time of day in the look-up table or by normalizing the outputs by first finding the anti-logarithm of the output equations and then applying a sigmoid function. A sigmoid function is a real valued differentiable function whose first derivative is bell shaped and has a single nonnegative or non-positive local maximum.

Though Lambrinos et al. in their robots have successfully demonstrated compass detection capability, an important disadvantage of their system is the use of three cameras. Three cameras with $0^{\circ}, 60^{\circ}$ and $120^{\circ}$ orientations have to be monitored which is expensive in terms of cost, area, complexity of the algorithm and also the computation power needed to implement the algorithms. Kane et al. [5.9] proposed a much simpler method where a normal camera with a linear polarization filter is used to take two images, the second image taken with the polarization filter set orthogonal with respect to its position in the first image. From these two images a mean intensity function as a function of polarization angle was derived similar to the form proposed in [5.7] for the photosensitive diodes.

To solve for the three unknowns in the equation (5.2) Kane et al. use three different images with the polarization transmission axis set to $0^{\circ}, 45^{\circ}$ and $90^{\circ}$ respectively. Rearranging equation (5.2) and accounting for images from the different transmission axis, three different image equations can be written.

$$
\begin{aligned}
& f_{1}(\varphi)=K I[1+d \cos (2 \varphi)] \\
& f_{2}(\varphi)=K I\left[1+d \cos \left(2 \varphi-2 \frac{\pi}{4}\right)\right] \\
& f_{3}(\varphi)=K I\left[1+d \cos \left(2 \varphi-2 \frac{\pi}{2}\right)\right]
\end{aligned}
$$

The set of equations is solved at each pixel of the image to compute the orientation angle $\varphi$.

This model also suffers from the disadvantage of using an external linear polarizer which needs to be rotated to have three images with three different transmission axis of the linear polarizer. 
Both the models of Lambrinos et al. and Kane et al. are also not very well compensated for the change in the degree of polarization during the day. The models also need an ephemeris compensation algorithm. The ephemeris function is a function that describes the change of the sun's azimuth over time and it depends on the season and the geographical latitude. It is known that the rate of change of the sun's azimuth is not constant: it is faster around noon and slower in the morning. Insects such as bees and ants also suffer from the inherent changes in the ephemeris function but are known to refine their compass detection ability with experience.

\subsection{Polarization based compass}

The models summarized in section 5.4 by Lambrinos et al. and Kane et al. are very similar. The former generates an output image by subtracting the individual image obtained by the two mutually orthogonal photodiodes while the latter generates an output image by adding the two mutually orthogonal images. In the designed image sensor described in this thesis, these functionalities can be easily incorporated because the individual pixels are polarization sensitive as shown in chapter 3.

\subsubsection{Measurement setup}

The measurement setup is the same described in chapter 3. The DC light source generates unpolarized light which is polarized by a linear polarizer. The linear polarized light intensity is then sensed by the imager and the analog output of the imager after digitization using an external $A D C$ is fed to the PC for processing. The corresponding analog outputs of the pixels sensitive to $0^{\circ}, 45^{\circ}$ and $90^{\circ}$ in the polarization sense regions are used to compute the Stokes parameters.

For the first version of the sensor the Stokes parameters were computed offchip to have a proof of concept. The circuits required for the computation of Stokes parameters and other digital processing for the implementation of the algorithm can be integrated on chip.

\subsubsection{Measurement results}

To validate the functionality as described in section 5.4, the pixel intensities for the $0^{\circ}$ and $90^{\circ}$ polarization orientation in the two polarization sense regions are recorded. The normalized pixel intensities for $0^{\circ}$ and $90^{\circ}$ polarization sensitive 
pixels in sense regions 1 and 2 are shown in figure 5.8. For both polarization sense regions the $0^{\circ}$ linear polarizer pixel has the maximum at $0^{\circ}$ and the minimum at $90^{\circ}$. Similarly, for the $90^{\circ}$ polarizer pixel the maximum is observed at $90^{\circ}$ and the minimum at $0^{\circ}$.

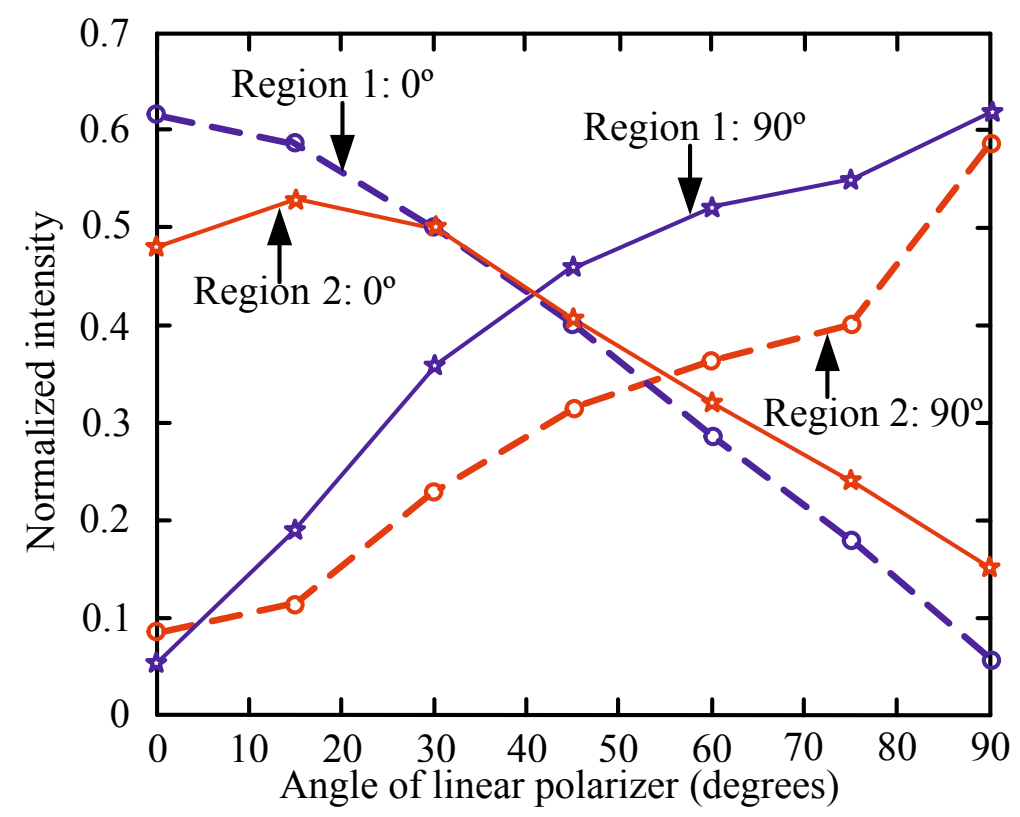

Figure 5.8: Normalized intensity obtained for $0^{\circ}$ and $90^{\circ}$ polarization.

To test the compatibility of the designed model with the model used by [5.7], the logarithmic difference of the mean intensities of the two linear polarizations was computed off chip and is plotted in figure 5.9. A very similar characteristic as the one shown by [5.7] and [5.8] was achieved for both sense regions.

For both polarization sense regions, the logarithmic difference of the two orthogonal signals was found to have a minimum at $0^{\circ}$ linear polarizer angle and a maximum at $90^{\circ}$ linear polarizer angle. The logarithmic difference shows an approximately linear increase for increasing linear polarizer angle [5.33]. This variation in the logarithmic difference can be conceptually used to determine the angle of linear polarization, and based on a reference or look-up table it can be used as a compass.

A different approach is taken here and instead of focusing on polarization differential image or polarization summation image, the variation in the degree of polarization with respect to the orientation of the $e$-vector is studied. The 


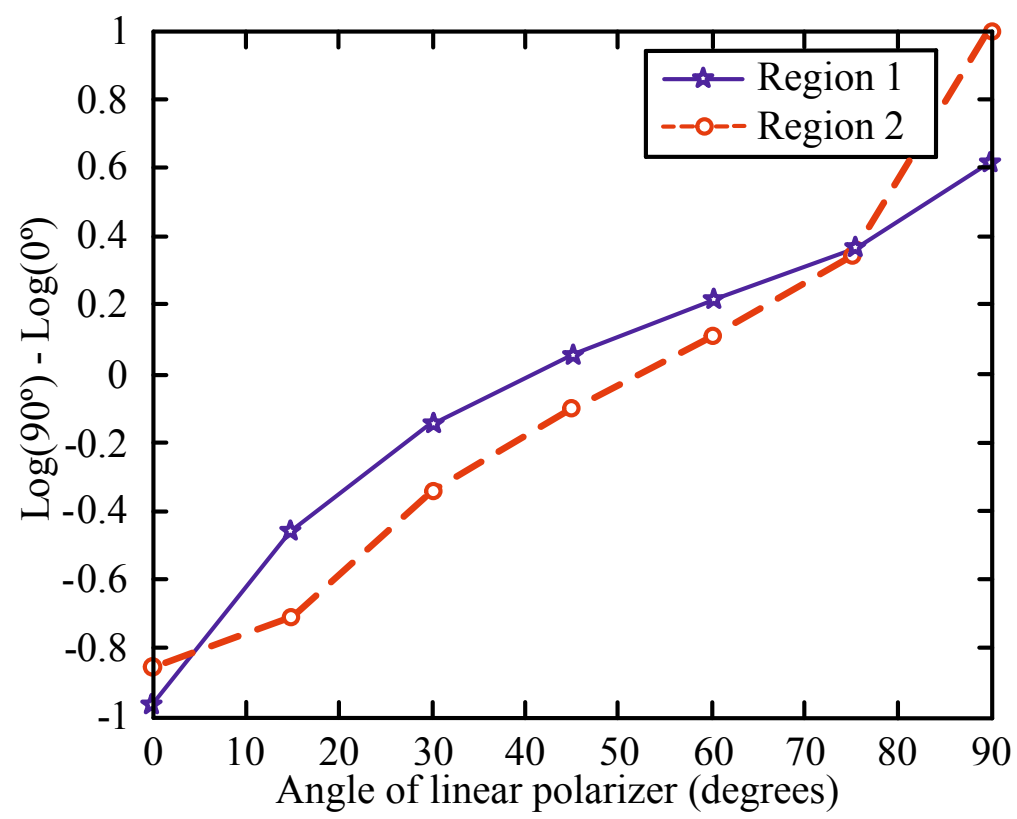

Figure 5.9: The logarithmic difference of the orthogonal signals in sense region 1 and 2.

image intensity from the photodiodes in the models described in section 5.4 is a function of the degree of polarization which changes over the course of the day. Lambrinos et al. offsets the effects of the variation in the degree of polarization by normalizing the output and Kane et al. did not compensate for it. The degree of polarization behavior with the orientation angle is evaluated and found that this degree of polarization information can be used to extract the orientation angle and hence can serve as a compass clue.

The variations in the degree of polarization in an image $(\varphi)$ for changes in the orientation angle $\varphi$ with respect to the solar meridian is given by equation (5.2). An inverse relationship between the degree of polarization $d$ and the orientation angle $\varphi$ is obtained.

$$
d=\frac{1}{\cos (2 \varphi)\left[1-\frac{f(\varphi)}{K I}\right]}
$$

Various methods of calculating the degree of polarization from the measured 
transmitted intensities through the implemented embedded metallic wire grid micro-polarizer have been discussed in chapter 3 and 4. The Stokes degree of linear polarization ( (3.17)) is shown in figure 5.10. The degree of polarization calculated using the maximum and minimum transmittance, equation (4.26), is shown in figure 5.11. Both plots show an inverse relationship between $e$-vector orientation angle and the degree of polarization. The Stokes parameters $S_{1}$ and $S_{2}$ used to calculate the Stokes degree of linear polarization are obtained as:

$$
\begin{aligned}
& S_{0}=I_{0^{\circ}}{ }^{2}+I_{90^{\circ}}{ }^{2} \\
& S_{1}=I_{0^{\circ}}{ }^{2}-I_{90^{\circ}}{ }^{2}
\end{aligned}
$$

where $I_{0^{\circ}}$ is the intensity of the light after passing through a horizontal linear polarizer, $I_{90^{\circ}}$ is the intensity after a vertical linear polarizer. Figures 5.10 and 5.11 show that both the DOLP obtained either by the Stokes parameters or by the $D O P$ obtained from the maximum and minimum transmittance vary between +1 to -1 as the polarizer angle is varied from $0^{\circ}$ to $90^{\circ}$ [5.33].

The variations in the degree of linear polarization with respect to the orientation angle can be used as a compass. This approach has the advantage of using fewer computations than the model proposed by Lambrinos et al., where at first the system output was logarithmized and then anti-logarithamized and normalized to remove the dependence on the degree of polarization.

The DOP in its simplest form is represented by the Polarization Fresnel Ratio $(P F R)$ described in section 4.3.5. The $P F R$ is the ratio of the perpendicular Fresnel coefficient to the parallel Fresnel coefficient. The Fresnel coefficients describe the reflection and transmission coefficients of the light wave at an interface. The $P F R$ can be roughly estimated as:

$$
P F R=\frac{P_{p e r}}{P_{p a r}}
$$

where $P_{p e r}$ is the power of the perpendicular polarization and $P_{p a r}$ is the power of the parallel polarization.

The pixel intensities obtained from the pixels covered with wire grid polarizer tilted by $0^{\circ}$ and $90^{\circ}$ in both sense regions are used to compute the PFR. The variation in the $P F R$ with varying angle of linear polarizer is shown in figure 5.12. 


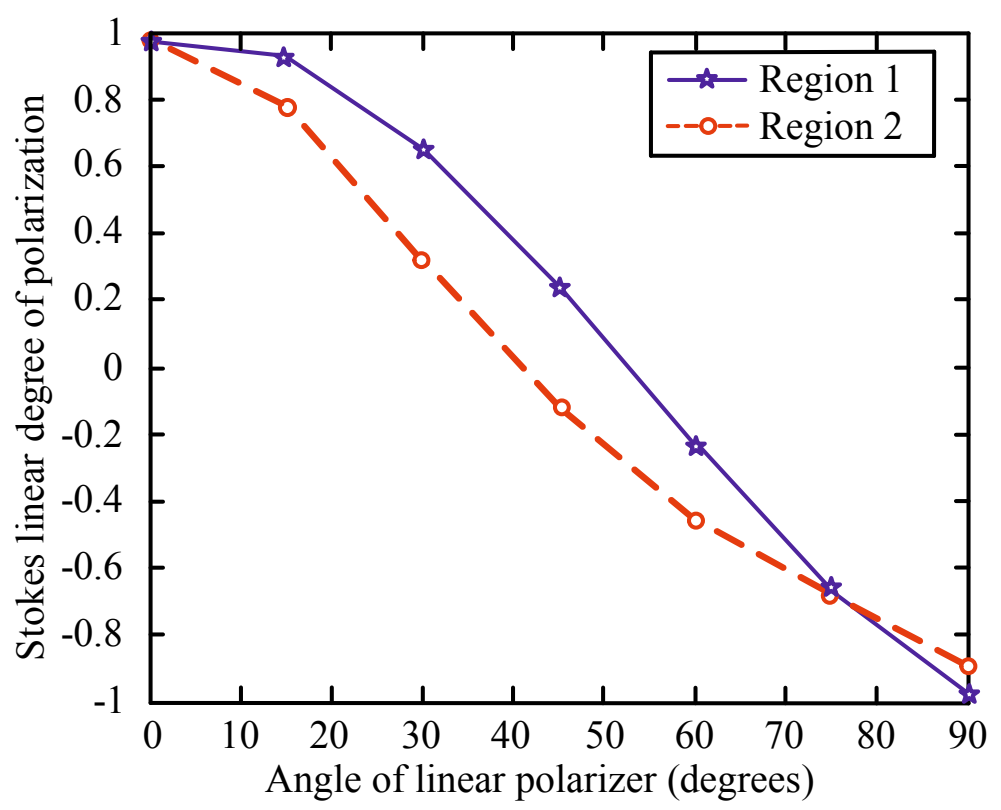

Figure 5.10: Stokes linear degree of polarization for sense region 1 and 2.

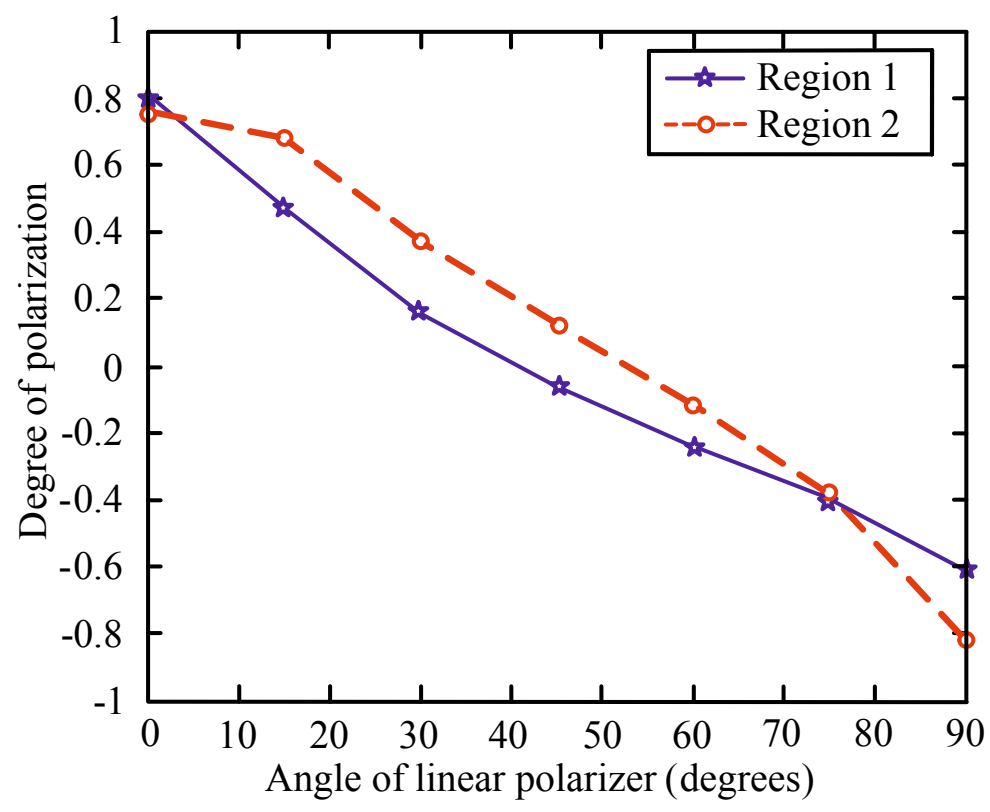

Figure 5.11: Degree of polarization for sense region 1 and 2. 


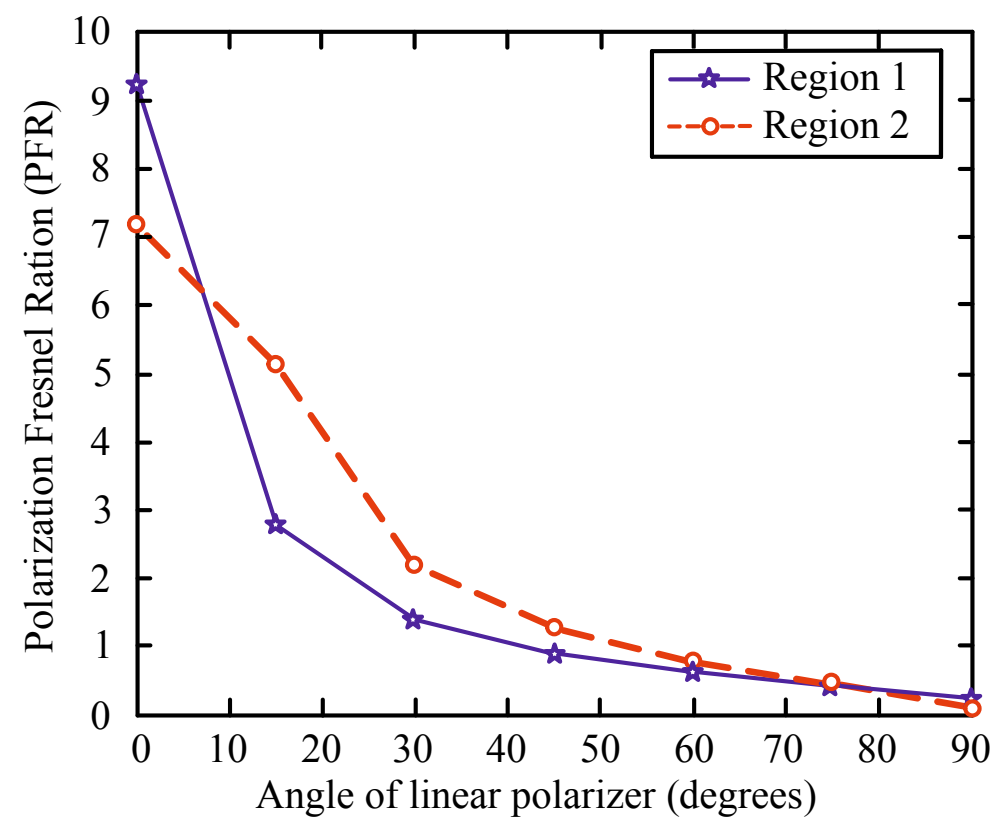

Figure 5.12: Polarization Fresnel Ratio (PFR) for sense region 1 and 2.

From figure 5.12, it is observed that the $P F R$ has a maximum value when the polarizer angle is $0^{\circ}$ and steadily decreases to a minimum when the polarizer angle is $90^{\circ}$ [5.33]. The decrease is steeper for a lower angle of the linear polarizer than for the higher values. Although the variations in the PFR with varying polarizer angle are not linear, the variation for various angles of linear polarizer is still suitable to be used as compass to detect the direction of the incoming polarized light ray.

\subsection{Incoming light ray direction detection and sun position detection}

In section 5.5 the variations of the degree of polarization with changes in the orientation angle were observed. In this section it is shown that it is also possible to compute the $e$-vector orientation angle from varying degree of polarization. A polarization navigation sensor principle which uses Stokes parameters to determine the incoming polarized light ray direction is described. 


\subsubsection{Measurement setup}

The experimental setup shown in figure 5.13 was built for indoor measurements using a DC light source.

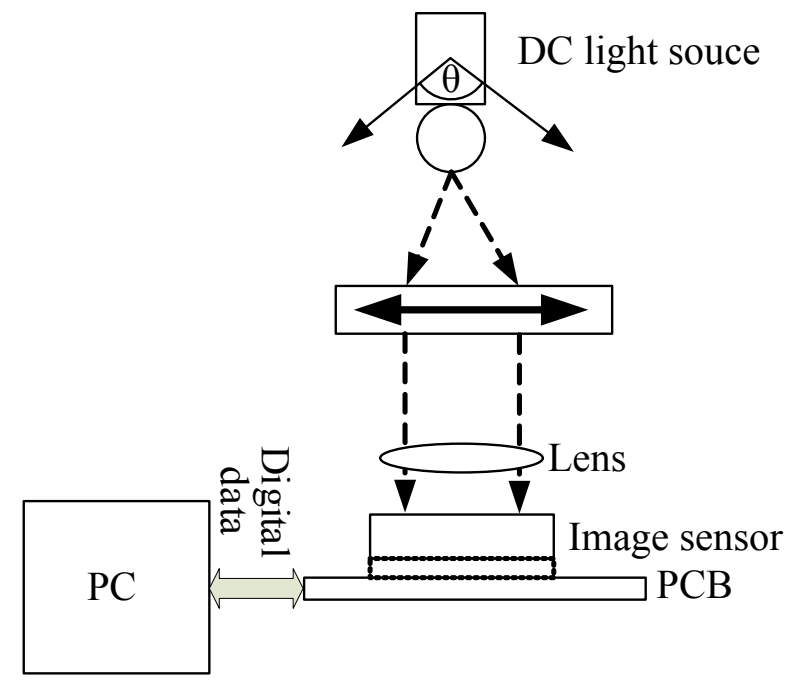

Figure 5.13: Measurement setup for incoming light ray direction.

The measurement setup is very similar to the one described in section 5.5.1, only here the DC light source is not fixed but it can be moved to have an angular direction. The sensor is illuminated with a polarized light obtained by passing the light from a DC light source through an external linear polarizer. The corresponding analog outputs of the pixels sensitive to $0^{\circ}, 45^{\circ}$ and $90^{\circ}$ in the polarization sense regions are recorded. These measurements are then sent to the PC for further processing. For the first version of the sensor the Stokes parameters were computed off-chip to have a proof of concept.

\subsubsection{Measurement results for incoming light ray directions}

The Poincare sphere described in chapter 3 is used to display the Stokes parameters and is characterized by an ellipticity angle and an azimuthal angle. The ellipticity angle and the azimuthal angle of the Poincar sphere, computed from the Stokes parameters are shown to be correlated with the incoming polarized light ray direction. 


\subsubsection{Incoming light ray direction detection using elliptic- ity angle}

To measure the ellipticity angle projected by the incoming light ray, the angular position of the DC light source in figure 5.13 was varied to change the incoming light ray direction. Using equations (3.16) and (3.19), the ellipticity angle can be expressed as

$$
\chi=\frac{1}{2} \sin ^{-1}\left(\frac{S_{3}}{\delta x S_{0}}\right)
$$

where $\chi$ is the ellipticity angle, $\delta$ is the degree of polarization and $S_{0}$ and $S_{3}$ are the Stokes parameters. The variation in the measured ellipticity angle with the variation in the angular position of the DC light source is shown in figure 5.14.

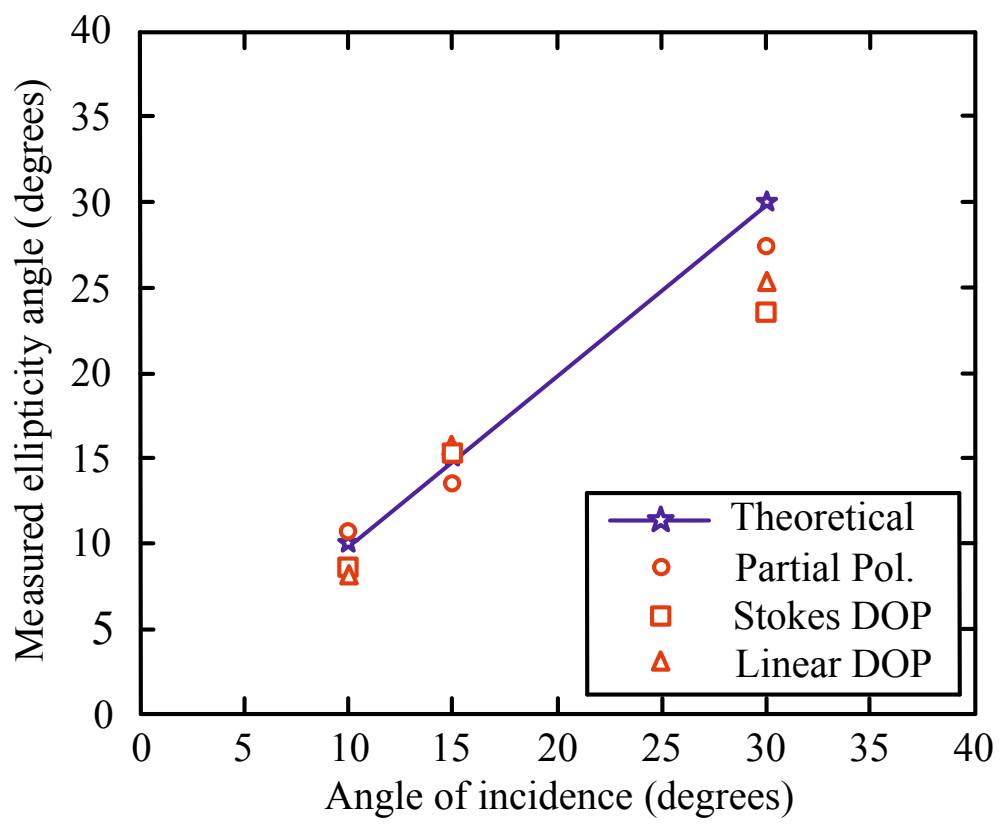

Figure 5.14: Measurement of incident light ray angle.

The ellipticity angle $(\chi)$ as shown in equation (5.12) depends on the degree of polarization. The measured ellipticity angle for $10^{\circ}, 15^{\circ}$ and $30^{\circ}$ incidence is shown as "Stokes DOP" in figure 5.14, where the degree of polarization is 
computed using the equation (3.16). The computation of the Stokes degree of polarization from the Stokes parameters needs squaring and square root arithmetic operations. These operations are relatively difficult to implement onchip.

The calculated ellipticity angle using the partial polarization computed from equation (4.31) is shown as "Partial Polarization" in figure 5.14. This computation is easier to implement on chip as it would only need a differential amplifier to compute the difference and an analog divider. A real time implementation model of an analog divider is discussed in section 5.7. In the skylight polarization only the linear component of the polarization is dominant, and the circular and elliptical polarization components are usually absent. Thus for experiments under open sky, only the linear degree of polarization will be measured. The measurement of the ellipticity angle using the Stokes degree of linear polarization as in equation (3.17) is shown in figure 5.14 as "Linear DOP".

The correlation coefficient indicates the strength and direction of a linear relationship between two random variables and can be calculated using equation (5.13)

$$
\text { correlation }=\frac{\left[N \sum x y-\sum x \sum y\right]}{\sqrt{\left[N \sum x^{2}-\left(\sum x\right)^{2}\right]\left[N \sum y^{2}-\left(\sum y\right)^{2}\right]}}
$$

where $N$ is the number of elements and $x$ and $y$ are the two variables.

Correlation coefficients of $0.98,0.94$ and 0.97 are obtained between the theoretical and measured results for Stokes DOP, Partial polarization and linear $D O P$ respectively [5.34]. The high values of the correlation coefficients imply a strong correlation between the theoretical and the measured results. Thus the incoming polarized light ray direction can be related to the ellipticity angle of the Poincaré sphere.

\subsubsection{Incoming light ray direction detection using az- imuthal angle}

The azimuthal position $(\psi)$ is computed from the Stokes parameters using equation (3.15) and (3.19). The theoretical and experimentally obtained azimuthal angle in the sense regions 1 and 2, or equivalently, the polarization angle measured while varying the transmission axis of the linear polarizer, is shown in the figure 5.15 .

The correlation coefficients of 0.997 and 0.98 in the two regions indicate a 


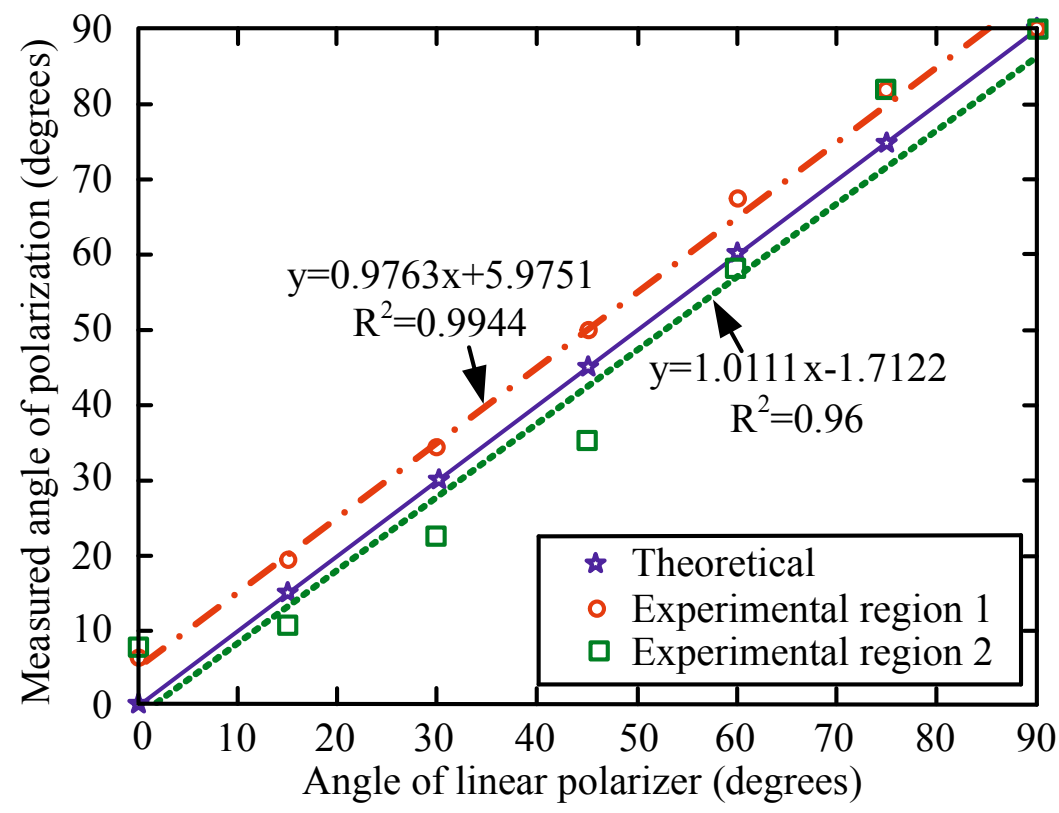

Figure 5.15: Measured angle of polarization in polarization sense region 1 and 2 using equations (3.15).

strong correlation between the theoretical and the measured results. A linear fit error for the measured angle of linear polarization is computed to be $2.3 \%$ and $1.1 \%$ in the sense region 1 and 2 respectively [5.35]. For a similar configuration using an organic micro-polarizer, the error is reported to be $2.2 \%$ [5.36] and an error of $1.6 \%$ is reported when aluminum nanowires is used with a wire grid pitch of 70nm [5.37] . The organic micro-polarizer exhibits a very high $E R$, thus the polarization intensity measurements are more accurate. The fabrication of these organic micro-polarizer's require very specialized processes, while the proposed method in this thesis uses standard CMOS technology processing steps and produces comparable results.

The polarization sense region 2 has an additional $45^{\circ}$ linear polarization sensitive pixel (figure 3.11). The Stokes equations are then modified to use the $45^{\circ}$ linear polarization information as: 


$$
\begin{aligned}
& S_{0}=I_{0^{\circ}}{ }^{2}+I_{90^{\circ}}{ }^{2} \\
& S_{1}=I_{0^{\circ}}{ }^{2}-I_{90^{\circ}}{ }^{2} \\
& S_{2}=I_{0^{\circ}}{ }^{2}-I_{45^{\circ}}{ }^{2}
\end{aligned}
$$

where $I_{0^{\circ}}, I_{90^{\circ}}$ and $I_{45^{\circ}}$ are the intensity of the light after passing through linear polarizer oriented at $0^{\circ}, 90^{\circ}$ and $45^{\circ}$. The fourth Stokes parameter $S_{3}$ is neglected as the light is assumed to be completely linearly polarized. The azimuthal position $(\psi)$ is then computed using equations (3.19) and is shown in figure 5.16.

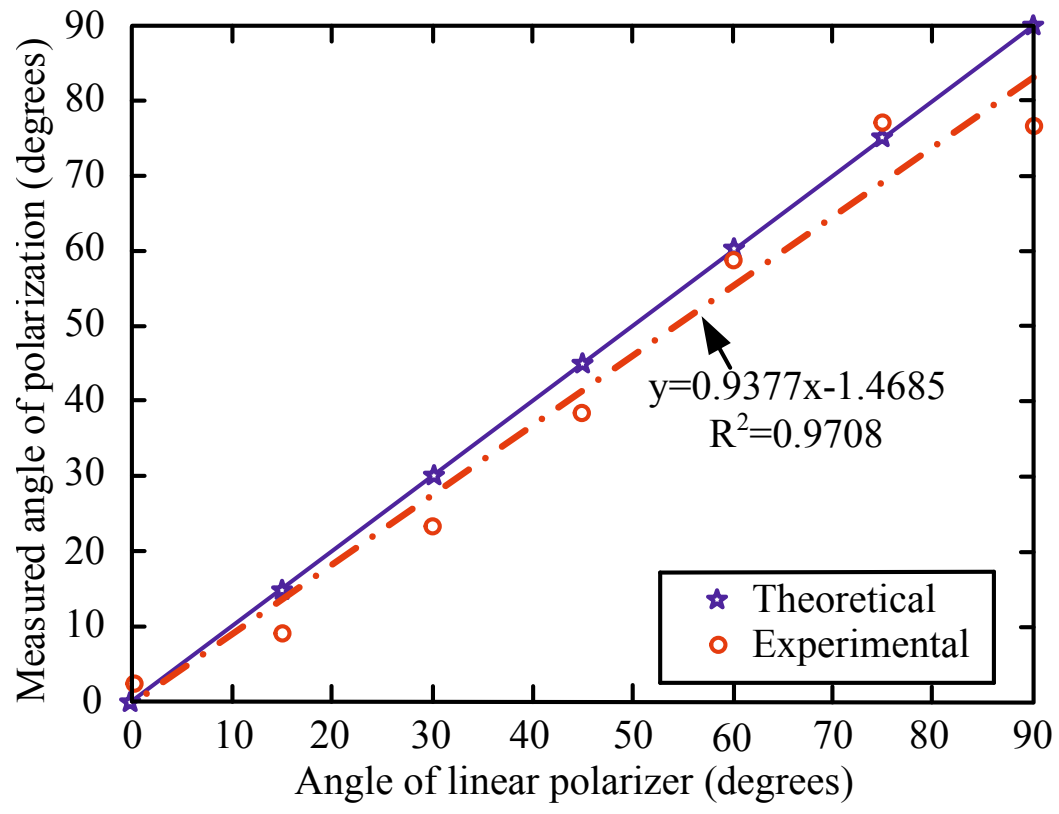

Figure 5.16: Measured angle of polarization in polarization sense 2 using equations (5.14).

A linear fit error for the angle of linear polarization measurements is computed to be $3.07 \%$. A correlation coefficient of 0.97 is obtained between the theoretical and the experimental values [5.35].

The Stokes parameters used to obtain the azimuthal position $(\psi)$ in figure 5.15 and figure 5.16 require the computation of the square of the pixel intensities, 
which is relatively difficult for an on-chip computation. To simplify the computation the Stokes equations can be further simplified as shown in equation (5.15).

$$
\begin{aligned}
& S_{m 0}=I_{0^{\circ}}+I_{90^{\circ}} \\
& S_{m 1}=I_{0^{\circ}}-I_{90^{\circ}} \\
& S_{m 2}=I_{0^{\circ}}-I_{45^{\circ}}
\end{aligned}
$$

where $S_{m 0}, S_{m 1}$ and $S_{m 2}$ are modified Stokes parameters, computed from the intensities. Equation (3.19) is again used to compute the azimuthal position $\psi$ and is plotted in the figure 5.17.

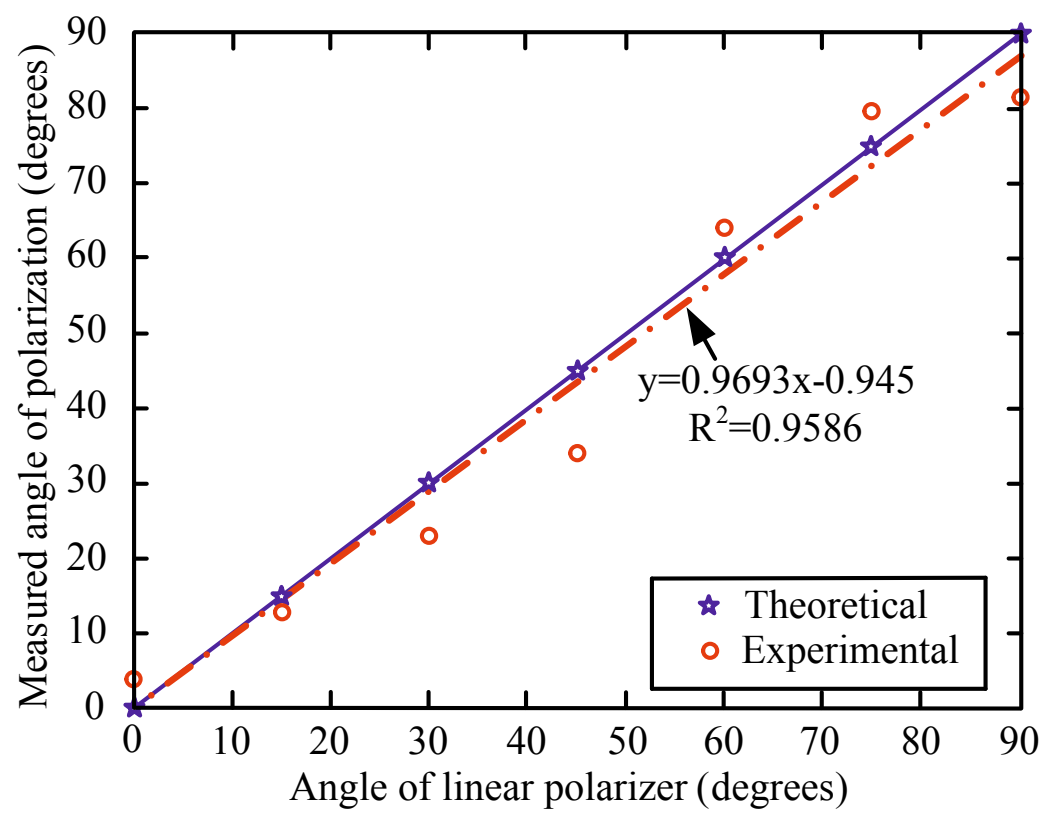

Figure 5.17: Measured angle of polarization in polarization sense region 2 using equations (5.15).

A linear fit error for the measured angle of linear polarization is computed to be $6 \%$. A correlation coefficient of 0.985 is obtained between the theoretical and the experimental values showing strong correlation [5.35]. The angular positional information obtained by equations (3.15) and (5.14) is very similar to those obtained by equations (5.15) as shown in the figures 5.16 and 5.17. The equations (5.15) are relatively easy to be implemented on-chip using simple 
operational amplifiers as analog adders or subtractors. The high linearity error is due to the higher metallic wire grid pitch used, limited by the choice of the process technology. With advances in the process technology the pitch can be considerably reduced providing better polarization detection ability and thus a lower linear fit error.

For figures 5.15, 5.16 and 5.17 the transmission axis of the linear polarizer was varied keeping the DC source fixed. The measured azimuthal angles for different angular position of the DC light source in the measurement setup (figure 5.13) for four different measurements are shown in figure 5.18. Similar to the ellipticity angle measurements, the angular position of the light source, and hence the angle of incidence of the light, was varied by $10^{\circ}, 15^{\circ}$ and $30^{\circ}$. A mean correlation coefficient of 0.9904 is obtained between the theoretical and the four measured results [5.38].

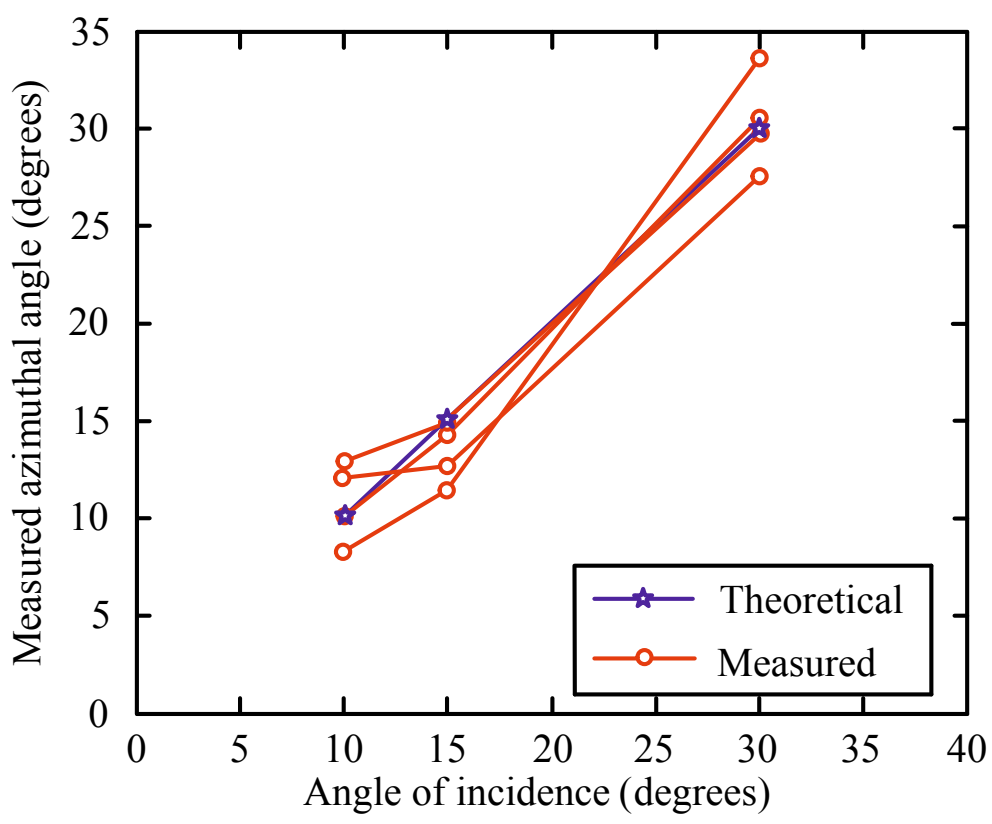

Figure 5.18: Measurement of incident light ray angle. 


\subsection{Real-time implementation of navigation com- pass}

In section 5.5, it was shown that the polarization Fresnel ratio (PFR) is a good tool to be used as a compass for autonomous agent navigations. To implement the $P F R$ calculations in real time using equation (5.11), an analog divider is needed. An analog divider can be implemented either in current mode or in voltage mode using a voltage controlled resistor as shown in figure 5.19 [5.39]. A current mode analog divider is discussed in this section. To use the current mode analog divider, the voltage at the floating diffusion node of the photodiode needs to be converted to current. This can be done using a transconductance amplifier or a $V$ - $I$ converter. If a conventional $p$ - $n$ junction photodiode is used then the photodiode current can directly be copied.

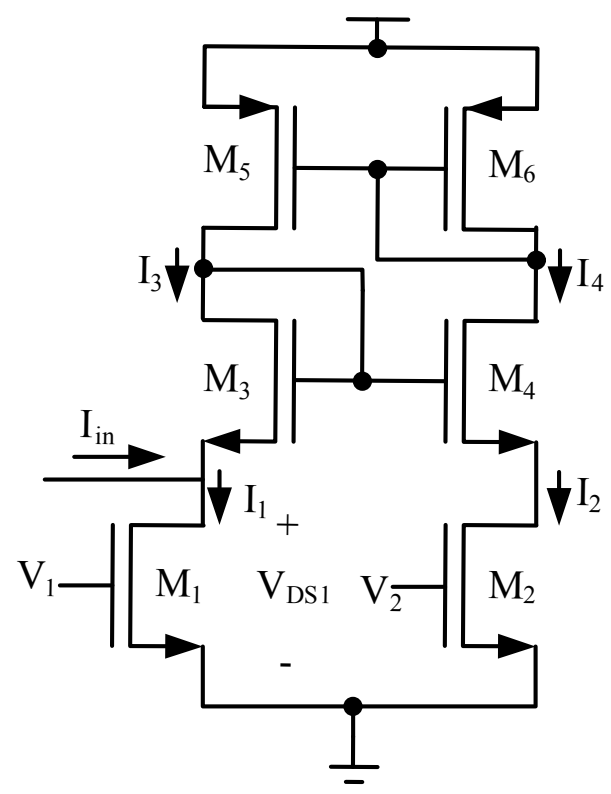

Figure 5.19: Voltage controlled resistor.

To implement an analog divider, a voltage controlled resistor $(V C R)$ is required, an implementation of $V C R$ is shown in figure 5.19. If transistors M1 and M2 are biased in triode region then the drain currents $I_{1}$ and $I_{2}$ are given by: 


$$
\begin{aligned}
& I_{1}=I_{i n}+I_{3}=\frac{K_{n 1}}{2}\left(2\left(V_{1}-V T H 1\right) V_{D S 1}-V_{D S 1}^{2}\right) \\
& I_{2}=\frac{K_{n 2}}{2}\left(2\left(V_{2}-V T H 2\right) V_{D S 2}-V_{D S 2}^{2}\right)
\end{aligned}
$$

where $k_{n 1}$ and $k_{n 2}$ are the transconductance parameters, $V_{1}$, and $V_{2}$ are the bias voltages, $V_{D S 1}$ and $V_{D S 2}$ are the drain to source voltage, $V_{T H 1}$ and $V_{T H 2}$ are the threshold voltages for the transistors $M_{1}$ and $M_{2}$ respectively, $I_{i n}$ is the additional injected current and $I_{3}$ is the drain current of transistor $M_{3}$.

The current mirror $M_{5}$ and $M_{6}$ ensures that $I_{3}$ and $I_{4}$ have the same value. The current $I_{4}$ also passes through the transistor $M_{2}$ so that

$$
I_{3}=I_{4}=I_{2}
$$

Assuming $M_{3}$ and $M_{4}$ to be perfectly matched and biased in saturation, $V_{G S 3}$ is expressed as:

$$
V_{G S 3}=V_{G S 4}=\sqrt{\frac{2 I_{2}}{K_{n 4}}}+V_{T H 4}
$$

Also

$$
V_{G S 4}+V_{D S 2}=V_{D S 1}+V_{G S 3}
$$

From equations (5.19) and (5.20)

$$
V_{D S 2}=V_{D S 1}
$$

Substituting equations (5.21), (5.18) and (5.17) in (5.16) and assuming the transistors $M_{1}$ and $M_{2}$ to be completely matched $\left(V_{T H 1}=V_{T H 2}=V_{T H}\right.$ and $\left.K_{n 1}=K_{n 1}=K_{n}\right), I_{i n}$ can be written as:

$$
I_{\text {in }}=K_{n} V_{D S 1}\left(V_{1}-V_{2}\right)
$$

From equation (5.22) a voltage controlled resistor between $I_{\text {in }}$ and ground can 
be realized with an equivalent resistance of

$$
R_{e q}=\frac{V_{D S 1}}{I_{\text {in }}}=\frac{1}{K_{n}\left(V_{1}-V_{2}\right)}
$$

Using the voltage controlled resistor of figure 5.19a current mode divider can be implemented as shown in figure 5.20 [5.40].

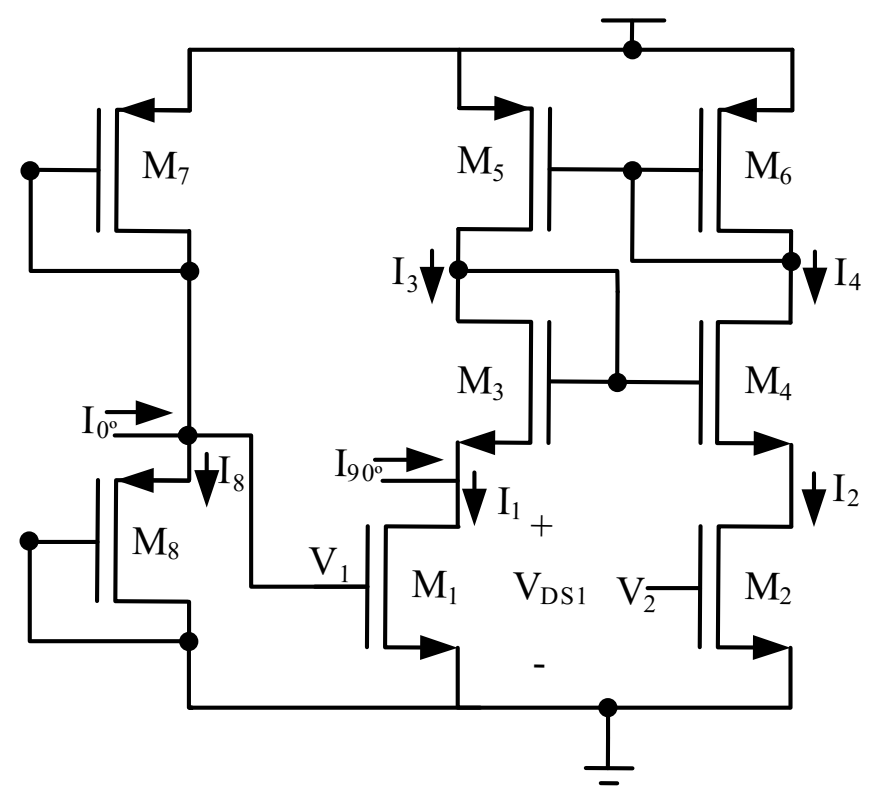

Figure 5.20: Current mode divider using voltage controlled resistor.

Assuming transistors $M_{7}$ and $M_{8}$ to be perfectly matched $\left(K_{p 7}=K_{p 8}=K_{p}\right.$ and $\left.V_{T H P 7}=V_{T H P 8}=V_{T H P}\right), V_{1}$ can be expressed as:

$$
V_{1}=\frac{I_{0^{\circ}}}{2 K_{p}\left(-V_{T H P}\right)}
$$

Substituting equation (5.23) in (5.21) and grounding $V_{2}$

$$
V_{D S 1}=\frac{2 K_{p}\left(-V_{T H P}\right)}{K_{n}} \times \frac{I_{90^{\circ}}}{I_{0^{\circ}}}
$$

According to equation (5.25) the output $V_{D S 1}$ is proportional to the ratio of the 
two input currents $I_{90^{\circ}}$ and $I_{0^{\circ}}$ which are the outputs of the two pixels sensitive to $0^{\circ}$ and $90^{\circ}$ sensitive pixels in the designed image sensor. This allows calculation of the PFR in continuous time.

Besides the requirement of an analog divider, the simplified Stokes parameter calculations in equation (5.15) also need a subtraction of the two pixel values which can be easily obtained using the circuit shown in figure 5.21.

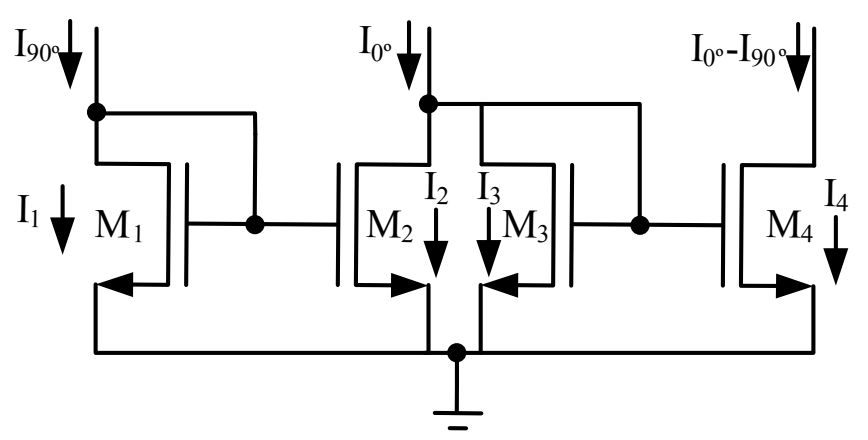

Figure 5.21: Current mode subtraction circuit.

If transistors $M_{1}, M_{2}, M_{3}$ and $M_{4}$ are in saturation, and assuming they are perfectly matched the currents in these two transistors are given by

$$
\begin{aligned}
& I_{1}=I_{90^{\circ}}=I_{2}=\frac{K_{n}}{2}\left(V_{G S 1}-V_{T H}\right)^{2} \\
& I_{3}=I_{0^{\circ}}-I_{90^{\circ}}
\end{aligned}
$$

Since $M_{3}$ and $M_{4}$ acts as a current mirror the current $I_{3}$ is copied by the transistor $M_{4}$ and the output current is the difference of the two input currents from the two pixels sensing $0^{\circ}$ and $90^{\circ}$ polarization.

\subsection{Conclusions}

A polarization based compass sensor for autonomous agent navigation using polarized light is described. The polarization angle can be calculated using the Stokes parameters, thus providing for a way to determine the incoming polarized light direction. This would in turn serve to determine the angular positional information useful for autonomous agent navigation. The computational algorithms are simplified for on-chip computations which would result in miniaturized navigational sensors. Such a navigational sensor would be independent of the 
visual cues and use natural light to determine the directional reference.

The polarization pattern of the skylight for a given elevation of the sun is a constant independent of the atmospheric disturbances, and this principle can be used as a compass clue. This can also be used to detect the position of the sun. In section 5.2 it was shown that the degree of polarization of the skylight depends on the position of the sun, and this variation in the degree of polarization can be used to compute the azimuthal and ellipticity angles of the Poincare sphere. Thus, the azimuthal and ellipticity angles calculated from the $D O P$ can be used to retrieve the position of the sun. This method would have an advantage over conventional sun position detection sensors as it would work even when the sun is not visible, and since only the degree of polarization needs to be computed, it would be easy to implement on chip. Due to the measurement setup constraints, it was not possible to measure the variations of the degree of polarization and the $e$-vector with respect to the angular position of the sun under open sky.

\subsection{References}

[5.1] J. O'keefe and L. Nadel, The Hippocampus as a Cognitive Map. Oxford University press, 1978. ISBN: 0198572069.

[5.2] P. Thorndyke and B. Hayes-Roth, "Differences in spatial knowledge acquired from maps and navigation," Cognitive Psychology, vol. 14, pp. 560-589, 1982.

[5.3] V. Hafner, Adaptive Navigation Strategies in Biorobotics: Visual Homing and Cognitive Mapping in Animals and Machines. Shaker Verlag, 2004. ISBN: 3832228578.

[5.4] R. Wehner, B. Michel, and P. Antonsen, "Visual navigation in insects: coupling of egocentric and geocentric information," Journal of Experimental Biology, vol. 199, pp. 129-140, 1996.

[5.5] H. Moravec, "Obstacle avoidance and navigation in the real world by a seeing robot rover," Technical Report CMU-RI-TR-3, Carnegie-Mellon University, Robotic Institute, 1980.

[5.6] G. DeSouza and A. Kak, "Vision for mobile robot navigation: A survey," IEEE Transactions on Pattern and Machine Intelligence, vol. 24, no. 2, pp. 237-267, 2002. 
[5.7] D. Lambrinos, R. Mller, T. Labhart, R. Pfeifer, and R. Wehner, "A mobile robot employing insect strategies for navigation," Robotics and Autonomous Systems, vol. 30, no. 1-2, pp. 39-64, 2000.

[5.8] D. Lambrinos, M. Maris, H. Kobayashi, T. Labhart, R. Pfeifer, and R. Wehner, "An autonomous agent navigating with a polarized light compass," Adaptive Behaviour, vol. 6, no. 1, pp. 131-161, 1997.

[5.9] K. Usher, P. Ridley, and P. Corke, "A camera as a polarized light compass: preliminary experiments," Proceedings of Australian Conference on Robotics and Automation, pp. 116-120, 2001.

[5.10] M. Mller and R. Wehner, "Path integration in desert ants, Cataglyphis Fortis," Proceedings of National Academy of Sciences, USA, vol. 85, pp. 5287-5290, 1988.

[5.11] Z. Chen and S. Birchfield, "Qualitative vision based mobile robot navigation," Proceedings of IEEE International Conference on Robotics and Automation, pp. 2686-2692, 2006.

[5.12] A. Moini, Vision chips. Boston: Kluwer academic publishers, 2002. ISBN: 0792386647.

[5.13] M. Mller and R. Wehner, "The hidden spiral: systematic search and path integration in desert ants, Cataglyphis Fortis," Journal of Comparitive Physiology A-Sensory Neural and Behavioural Physiology, vol. 175, pp. 525-530, 1994.

[5.14] R. Wehner and M. Mller, "The significance of direct sunlight and polarized skylight in the ant's celestial system of navigation," Proceedings of the National Academy of Sciences, USA, vol. 103, no. 33, pp. 1257512579, 2006.

[5.15] F. Santschi Review Suisse Zoology, vol. 19, pp. 303-338, 1911.

[5.16] C. Liebe, "Solar compass chip," IEEE Sensors Journal, vol. 4, no. 6, pp. 779-786, 2004.

[5.17] Y. Chang, S. Kang, and B. Lee, "High accuracy image centroding algorithm for CMOS based digital sun sensors," Proceedings of IEEE Sensors Conference, pp. 329-336, 2007.

[5.18] N. Xie, A. Theuwissen, and X. Wang, "A CMOS image sensor with row and column profiling means," Proceedings of IEEE Sensors Conference, pp. 1356-1359, 2008. 
[5.19] L. Bjorn, Photobiology: the science of life and light, p. 18. Springer publications, 2007. ISBN: 9780387726540.

[5.20] K. Coulson, Polarization and Intensity of Light in the atmosphere, p. 2. Deepak, Hampton, VA, 1988. ISBN: 0937194123.

[5.21] G. Smith, "The polarization of skylight: An example from nature," American Journal of Physics, vol. 75, no. 1, pp. 25-35, 2007.

[5.22] R. Richardson and E. Hulburt, "Sky-brightness measurements near bocaiuva, brazil," Journal of Geophysics Research, vol. 54, pp. 215-227, 1949.

[5.23] R. Wehner, "Desert ant navigation: how miniature brains solve complex task," Journal of Comparitive Physiology A-Sensory Neural and Behavioural Physiology, vol. 189, pp. 579-588, 2003.

[5.24] T. Seidl, "Ant navigation and path finding," Proceedings of the 2nd ACT Workshop on Innovative Concepts, vol. 31, no. 4, pp. 102-110, 2008.

[5.25] H. Pieron, "Du role du sens musculaire dans l'orientation de quelques espces de fourmis," Bulletin of Institute of General Psychology, vol. 4, pp. 168-186, 1904.

[5.26] V. Cornetz, "Trajets de fourmis et retours au nid," Mmoire De l'Institute Gneral, Psychologie, vol. 2, pp. 1-67, 1910.

[5.27] M. Mittelstaedt and H. Mittelstaedt, "Homing by path integration in a mammal," Naturwissenschaften, vol. 67, pp. 566-567, 1980.

[5.28] R. Wehner, "The ant's celestial compass system: spectral and polarization channels," Orientation and Communication in Arthropods, Basel, Birkhuser Verlag, pp. 145-185, 1997.

[5.29] T. Labhart and E. Meyer, "Detectors for polarized skylight in insects: a survey of ommatidial specializations in the dorsal rim area of the compound eye," Microscopy Research and Technique, vol. 47, pp. 368-379, 1999.

[5.30] P. Herrling, "Regional distribution of three ultrastructural retinula types in the retina of cataglyphis bicolor fabr. (formicidae, hymenoptera)," Cell Tissue Research, vol. 69, pp. 247-266, 1976.

[5.31] T. Labhart, "How polarization-sensitive interneurons of crickets see the polarization pattern of the sky: a field study with an opto-electronic model neuron," Journal of Experimental Biology, vol. 202, pp. 757-770, 1999.

[5.32] G. Bernard and R. Wehner, "Functional similarities between polarization vision and color vision," Vision Research, vol. 17, pp. 1019-1028, 1977. 
[5.33] M. Sarkar, D. S. Segundo, C. van Hoof, and A. Theuwissen, "Integrated polarization analyzing CMOS image sensor for autonomous agent navigation using polarized light," Proceedings of IEEE International conference on Intelligent Systems, pp. 224-229, 2010.

[5.34] M. Sarkar, D. S. Segundo, C. van Hoof, and A. Theuwissen, "Integrated polarization analyzing CMOS image sensor for detecting incoming light ray direction," Proceedings of IEEE Sensors Application Symposium, pp. 194199, 2010.

[5.35] M. Sarkar, D. S. Segundo, C. van Hoof, and A. Theuwissen, "Biologically inspired autonomous agent navigation using stokes parameters and an integrated polarization analyzing CMOS image sensor," Proceedings of Eurosensors XXIV conference, vol. 5, pp. 673-676, 2010.

[5.36] V. Gruev, J. van der Spiegel, and N. Engheta, "Integrated polarization image sensor for cell detection," International image sensor workshop, 2009.

[5.37] V. Gruev and R. Perkins, "A 1 mpixel ccd image sensor with aluminum nanowire polarization filter," Proceedings of IEEE International Symposium on Circuits and Systems, pp. 629-632, 2010.

[5.38] M. Sarkar, D. S. Segundo, C. van Hoof, and A. Theuwissen, "Integrated polarization analyzing CMOS for navigation and incoming light ray direction," IEEE Transactions of Instrumentation and Measurement. (accepted for publication).

[5.39] W. Liu and S. Liu, "CMOS tunable 1/x circuit and its applications," Transactions on Fundamentals of electronics, communications and computer sciences, vol. E-86A, pp. 1896-1899, 2003.

[5.40] W. Liu, S. Liu, and S. Wei, "CMOS current-mode divider and its application," IEEE Transactions on Circuits and Systems-II, vol. 52, no. 3, pp. 145-148, 2005. 


\section{6}

\section{Motion detection and digital polarization}

Flying insects have extraordinary visual capabilities. Their ability to detect fast motion in the visual scene and avoid collision using low level image processing and little computational power makes their visual processing interesting for real time motion/collision detection in machine vision applications.

In this chapter, some bio-inspired models of motion and collision detection based on differential imaging and correlation will be presented. Section 6.1 describes the process of obtaining motion from a sequence of images. The conventional models for motion detection using differential imaging, background subtraction and optical flow are also briefly introduced. Motion detection using differential imaging is described in section 6.2. Section 6.3 describes the most popular bio-inspired elementary motion detector. It also discusses on the possible simplification of elementary motion detection using one-dimensional binary optical flow. The spatial summation of differential one-dimensional binary optical flow is shown to be able to detect both horizontal and vertical object motion. The partial charge transfer method to increase the dynamic range and the background illumination invariance are described in section 6.4. A digital representation of polarization is presented in section 6.5 , where the onedimensional binary optical flow is shown to vary with the polarization angle of the incoming light rays. 


\subsection{Motion detection}

Motion is usually determined from image sequences. The spatiotemporal image sequences can be represented using the plenoptic function. The plenoptic function was introduced by Adelson and Bergen [6.1] to describe all the information available to an observer at any point in space and time, when a light ray passes through the imaging device. In its most general form, the plenoptic function is a seven-dimensional function given by the equation (6.1)

$$
P=P\left(\theta, \varphi, t, \lambda, V_{x}, V_{y}, V_{z}\right)
$$

where $\left(V_{x}, V_{y}, V_{z}\right)$ is the position of the imaging sensor in three-dimensional space, $\lambda$ is the wavelength of light, $(\theta, \varphi)$ represents the azimuth and elevation angles that index the viewable rays and $t$ is time. In a pinhole camera only one sample of the viewpoint is available at any given instance, thus the plenoptic function can be adapted to standard Cartesian parametrization of the rays $(x, y)$, where $x$ and $y$ are the spatial coordinates in the image plane.

$$
P=P(x, y, t, \lambda)
$$

Though multispectral imaging has started to become popular, most motion analysis are done for a single wavelength and thus the plenoptic function can be rewritten as

$$
P=P(x, y, t)
$$

The plenotic function of a time sampled set of images or "snapshots" is denoted by

$$
P=P(x, y,\{t=i, i+\delta t, \ldots, i+n \delta t\})
$$

where $i$ is the time instance of an image capture, $\delta t$ is the time interval between two consecutive images and $n$ is total number of images captured. In machine vision, the variations in the plenotic function across sequences of images are used to calculate the image velocity or motion. One of the measurable parameters of a point object which is assumed not to change with time for changing plenoptic function is the intensity or the brightness of the object given by $I(x, y, t)$, where $(x, y)$ represents the spatial $x$ and $y$ dimensions and $t$ denotes time [6.2]. It is 
thus useful to combine the geometrical description of the scene with brightness information for motion detection [6.3]. The brightness constancy equation assuming the intensity of a point to remain constant as it moves $\delta x, \delta y$ for a time interval $\delta t$ can be written as

$$
I(x-\delta x, y-\delta y, t-\delta t)=I(x, y, t)
$$

Taylor series expansion of equation (6.5) can be written as:

$$
\begin{aligned}
I(x-\delta x, y-\delta y, t-\delta t)=I(x, y, t)+\frac{\partial I}{\partial x} \delta x & +\frac{\partial I}{\partial y} \delta y+\frac{\partial I}{\partial t} \delta t \\
& + \text { higher order terms }
\end{aligned}
$$

Neglecting the higher order terms in equation (6.6) and using the brightness constancy equation [6.1] we get

$$
\frac{\partial I}{\partial x} \delta x+\frac{\partial I}{\partial y} \delta y+\frac{\partial I}{\partial t} \delta t=0
$$

where $\partial I / \partial x, \partial I / \partial y$ and $\partial I / \partial t$ are the derivatives of the image $(x, y, t)$ in the corresponding directions and thus can be represented by $I_{x}, I_{y}$ and $I_{t}$. Equation (6.7) can be rewritten as:

$$
I_{x} \delta x+I_{y} \delta y+I_{t} \delta t=0
$$

Dividing all the terms in equation (6.8) by $\delta t$ we get:

$$
I_{x} u+I_{y} v+I_{t}=0
$$

where $u(x, y, t)$ and $v(x, y, t)$ are the horizontal and vertical components of the motion respectively.

Equation (6.9) relates the image velocity to the spatiotemporal derivative of the image at a particular location and is commonly referred to as the motion constraint equation.

Based on the variation in the intensity obtained from the projection of the plenoptic function on the image sensor from the moving object, three conventional approaches are used to detect motion: temporal differencing [6.4]; 
background subtraction [6.5], [6.6]; and optical flow [6.2].

Temporal differencing is based on frame difference, and attempts to detect moving regions by making use of the difference of consecutive frames (two or three) in a video sequence. This method is highly adaptive to dynamic environments, but generally does a poor job of extracting the complete shapes of certain types of moving objects [6.7].

Background subtraction uses a model of the background and compares the current image with the reference image to separate the background and foreground [6.8]. The main disadvantage of background subtraction method is that both the background scene and the camera are required to be stationary when this method is applied. They are also extremely sensitive to dynamic scene changes due to background illumination changes.

When either the optical scene or the camera is in motion, optical flow is usually used. An object in motion may exhibit both translation and rotational velocities, which are usually projected as the movement of brightness patterns on the image plane. As per the smoothness constraints, the corresponding points in two successive frames should not move more than a few pixels. Thus in an uncertain environment the camera motion or background changes should be relatively small and thus motion can still be detected with optical flow. Optical flow estimation methods can be classified into three main groups: differential methods; matching-based methods and frequency/phase-based. All the three methods consist of three basic components: pre-filtering, local motion estimation and integration over the field of view. Pre-filtering or smoothing of the image data with a low-pass or band-pass filter allows extracting the signal of interest thus enhancing signal-to-noise ratio. The local motion estimation is done using spatiotemporal derivatives to measure the velocity component and the integration over the field of view produces a two-dimensional flow field of the moving object.

The differential methods include the gradient based models that determine two-dimensional velocities of the moving object employing first or second order spatiotemporal derivatives of the image sequence. The velocity is obtained by dividing the temporal derivative of local luminance by its spatial derivative. The major problem with gradient based methods is that if the spatial derivative is small, the noise in the temporal derivative is amplified and the velocity is poorly defined. Thus due to the differentiation of the image sequence and their susceptibility to errors under noisy conditions they are usually not preferred in low signal-to-noise ratio regimes [6.9].

Matching-based methods include feature-based and region-based methods. They are also known as block-based, area-based and correlation based methods. 
These methods usually use techniques to either maximize cross-correlation or minimize differential error. Feature based methods locate and trace identifiable features of the image over time while region based methods try to locate a delineated region in consecutive frame within a search space. To match the feature or the regions in subsequent images either probabilistic approaches like Kalman filters and Monte Carlo localizations or neural networks are used. Matching based methods are robust to large motion and brightness variations however these methods are found to be accurate only at high velocities and it is difficult to estimate sub-pixel displacements [6.10]. Further correlation based models are normally very sensitive to the amount of data involved because these algorithms are mainly based on byte-level operations of the whole image. These methods involve an operation of all the pixels in the image even though most pixels may not have changed from one frame to another, severely limiting the bandwidth and speed or motion detection for real-time applications.

Frequency/phase based methods use local energy or phase information to determine the velocity of the moving object. These techniques for determining image motion rely on the phase behaviour of arrays of band-pass filters or changes in the output energy of the velocity tuned filters. These filters decompose the input signal according to scale, speed and orientation. However these methods are still susceptible to noise and discontinuity limitations like the gradient based methods. Additionally the outputs of these methods are limited by the design of the filters used [6.10].

\subsubsection{Motion detection - models}

The problem of real-time motion detection and tracking is an important issue in artificial vision. The main constraints for real-time implementation of these algorithms are the large amount of data to be processed and the highcomputational cost of the algorithms employed. To solve the problems, analog VLSI chips employing early vision processing of the optical scene are becoming popular [6.11], [6.12], [6.13], [6.14]. They employ simple, low accuracy operations at each pixel in an image or sequence of images, resulting in a lowlevel description of a scene useful for higher level machine vision applications. These often results in compact, high speed and low power solutions.

The motion detection can be done either on a pixel level or on a region level. The pixel level analysis shows whether the pixel is stationary or transient by observing its intensity value over time. Region analysis deals with the agglomeration of groups of pixels into moving and stationary regions. 
It is usually known that the pixel's intensity value displays three characteristic profiles as shown in the figure 6.1. When an object moves through the pixel it displays a profile that exhibits a step change in intensity, followed by a period of instability, then another step back to the original background intensity. When the object moving through the pixel stops at the pixel location, it exhibits a change in intensity, followed by a period of instability and then settling to the new intensity levels. With variations in the ambient lighting, the intensity changes smoothly with no large steps [6.15]. To know the state of the object, the nature of the pixel intensity profile is important. To interpret the meaning of a step change (for example object passing through, stopping at, or leaving the pixel), one needs to observe the intensity curve re-stabilizing after the step change. This introduces a time-delay into the process. For detection of fast motion this time delay has to be minimized.
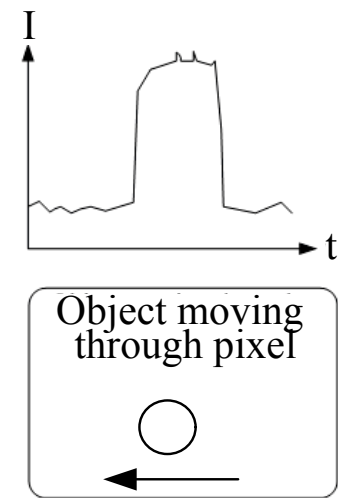

(a)
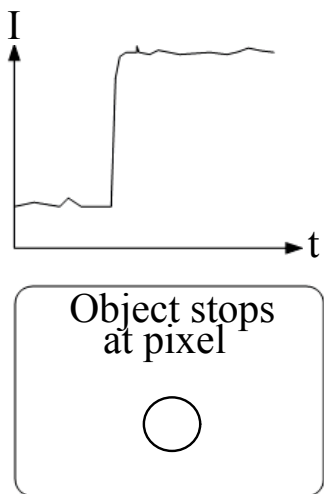

(b)
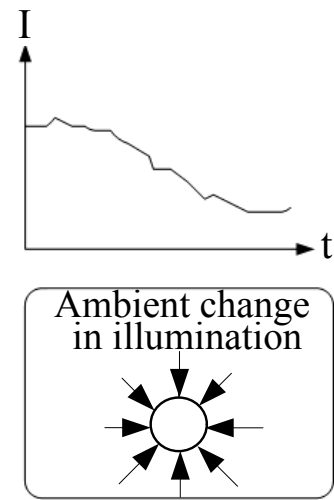

(c)

Figure 6.1: Characteristic pixel intensity profiles for (a) object moving though the pixel, (b) object stopping at the pixel and (c) variations in the ambient illumination changes.

In motion detection algorithms where the image motion is analyzed by sending the complete frame out of the image sensor, a very high data rate is required to keep the time delay small. The common method employed to obtain differential images is to use the CCD or CMOS image sensor in high speed frame mode combined with fast readout, a frame buffer to store the frame and a digital subtractor to generate the differential image. The high frame rate is needed to prevent temporal aliasing, which prevents the output signal from being a smooth function of the image parameters thus affecting the efficiency of motion detection algorithms. Furthermore a long time between two image captures will give a 
difficult correspondence problem in a dynamic scene. Such an image sensor is highly complex and consumes lot of power. Thus such motion sensing algorithms though in use, for example in MPEG encoders, are not very convenient for realtime motion applications in machine vision. These algorithms for fast motion detection become design overkill for slow motion detections where the time delay can be large.

Temporal differencing at a pixel level rather than at a frame level can help to decrease the data coming from the camera as only the data from the pixel with changed states will be sent out [6.12], [6.16]. The focal plane computations are also free from temporal aliasing, which is usually a problem in motion detection algorithms. Further temporal differencing is useful for ambient light suppression. Two methods are discussed, one analog and one digital, in the subsequent sections that can be employed for speeding up low level motion detection without much complexity and power consumption. These algorithms are based on pixel changes instead of full image processing and thus improve performance. The pixel level processing provides an extreme data rate compression; it has a high data rate in (image sequence) and low data rate out (motion).

\subsection{Motion detection - differential imaging}

In differential imaging two consecutive images are stored in the pixel as an analog voltage and a difference signal is obtained during the readout. Differential imaging can be used for machine vision applications like motion detection, object tracking or object recognition etc. All the stationary objects with constant illumination will be ignored by the imaging system.

An active differential sensing method is described here, with focal plane computations which need minimal external active components. The subtraction of the two images captured at varying exposure time is done pixel by pixel. The process is repeated for each image line and thus a differential image is obtained without a requirement for an off-chip computation.

The designed image sensor explained in chapter 2 can operate in two modes: double differential mode $(D D S)$ and differential imaging mode $(D I)$ using the two available analog memories in the pixel. The operation of the image sensor in the $D D S$ mode was shown in section 2.5.5.1. The pixel diagram with the two analog memories and the operational timing diagram of the DI mode of the pixel are shown in the figure 6.2.

In the $D I$ mode the sensor takes two samples and subtracts one from the other. The first sample is taken after an integration time $T_{1}$ while the second sample is 

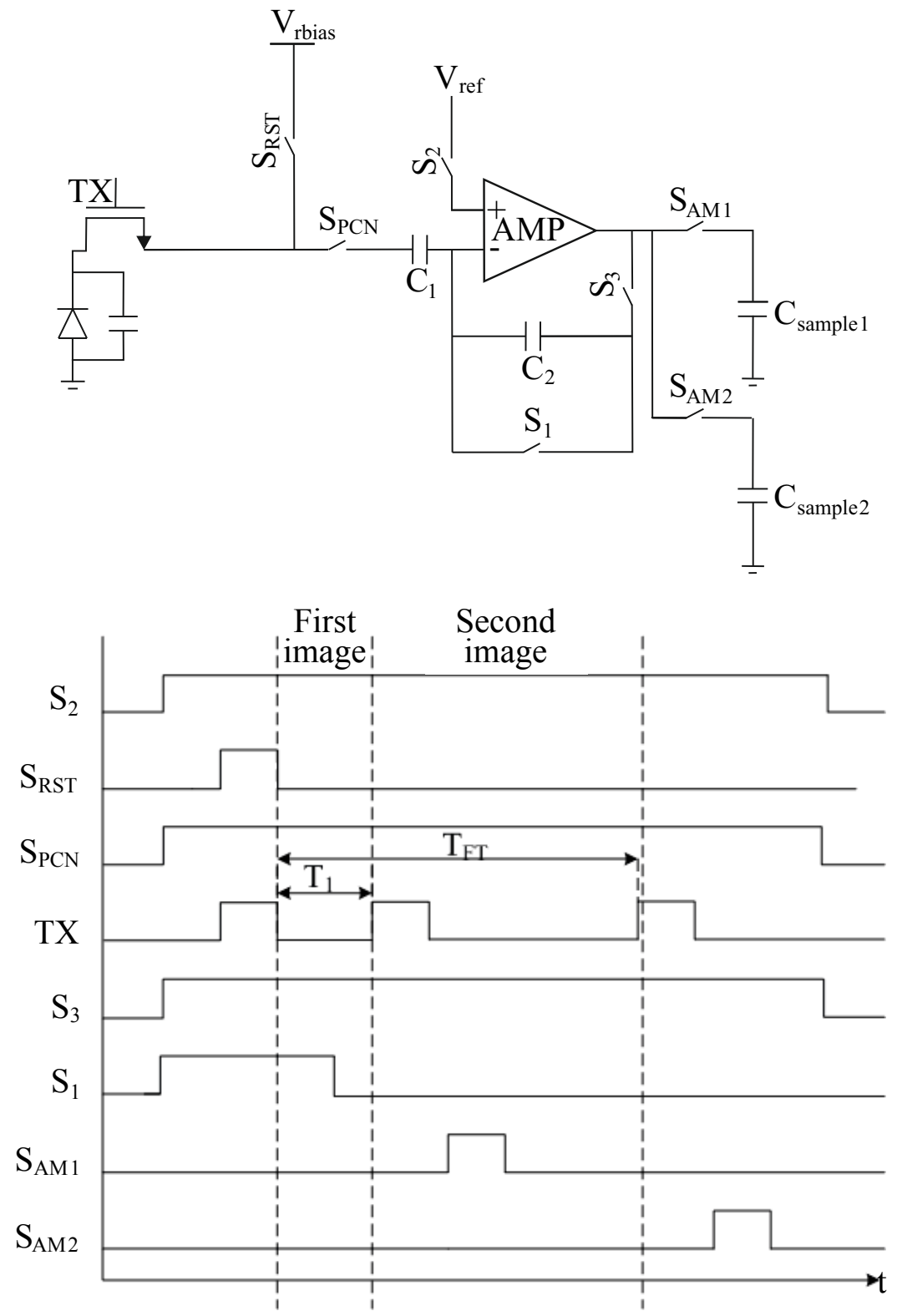

Figure 6.2: Differential imaging mode.

taken after an integration time $T_{F T}$ and are stored in the two analog memories in the pixel. The imaging array operates in global shutter mode as the signals 
after the integration time is transferred to the memories at the same time for all the pixels of the imaging array. However while reading out, each row is read at a time. The two signals stored in the pixel are subtracted at the column level of the analog signal chain shown in figure 2.27. The subtraction of the two images reduces the FPN and since there is no reset between the two image captures the reset noise is the same in both images and it is removed with subtraction.

Figure 6.3 shows an example of motion detection, when the object moves to a pixel position and stops, similar to the situation shown in figure 6.1(b).
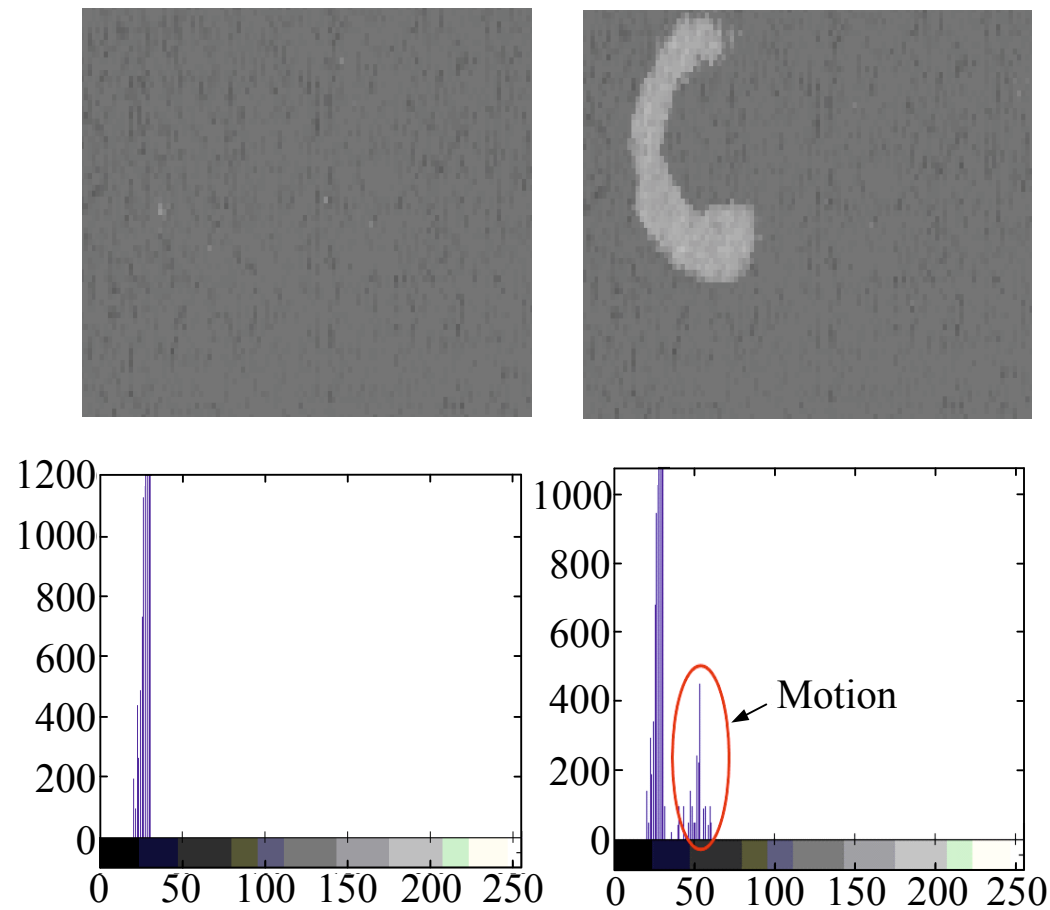

Figure 6.3: Motion detection using differential mode of operation.

When the exposure time of the two samples is the same $\left(T_{1}=T_{F T}\right)=20 \mathrm{~ms}$, a black image is observed when there is no change in the background as shown in figure 6.3(a). When a DC light source is switched on (to resemble motion), there is a change in the background and the change is visible in the differential image 6.3(b). The histograms shows the gray values of the pixels. When there is no motion, most of the pixels have lower gray values while when there is motion pixels start to get higher gray values.

For motion detection all the static objects or DC light sources in the background have to be removed and only the AC change is to be detected. Thus 
a very high Common-Mode Rejection Ratio $(C M R R)$ is desired to remove the common mode or DC signal in subsequent images of a scene. Common-mode rejection ratio of a device is the measure of the tendency of the device to reject common input signal. A high $C M R R$ is desired for applications where the signal of interest is a small voltage fluctuation superimposed on a large voltage offset or when the required information is contained in the voltage difference of two signals.

The definition of the CMRR of the differential image is simplified from the conventional definition of $C M R R$ and defined to be the change in the common mode signal divided by the change in the differential signal due to the change in the common mode signal [6.17]. A high CMRR is desired to have the same effect when two white images or two black images are subtracted from each other. The maximum voltage swing obtained after $D D S$ for the designed sensor is $488 \mathrm{mV}$ as seen in section 2.6.2.1, for dark to saturation exposure of the sensor. The output voltage swing obtained when operating in the differential mode $\left(T_{1}=T_{F T}\right)$ is $2.44 \mathrm{mV}$ for varying illumination from dark till the sensor saturation. The $C M R R$ thus obtained is 200 or $46 \mathrm{~dB}$ which is higher than the CMRR of 100 reported in [6.17].

The changes in the differential signal when the normal exposure period $\left(T_{F T}\right)$ is held constant at $20 \mathrm{~ms}$ while the short integration time $\left(T_{1}\right)$ is varied is shown in the figure 6.4 .

A non-linear behavior is seen when $T_{1}$ is very low compared to $T_{F T}$. If the ratio of the two integration periods is defined as $R_{D E}=T_{F T} / T_{1}$, then it is observed that for larger values of $R_{D E}$ the non-linearity is quite high. The accumulated electrons for a given illumination condition decrease with the increase in $R_{D E}$ due to shorter accumulation period (The accumulated electrons are proportional to $\left.1 / R_{D E}\right)$. When the accumulated electrons are low, the error in transferring these accumulated electrons from the photodiode to the floating diffusion node is higher. Thus the non linearity increases with increase in $R_{D E}$. The results are very much in agreement with the theoretical studies of [6.18]. The CMRR practically remains low for all values of $T_{1}$. When $T_{1}$ is equal to $T_{F T}$, it reaches the maximum value of 200 .

\subsection{Motion detection - optical flow}

A one-dimensional motion can either be in a horizontal direction or in a vertical direction. Vertical motion of an object towards the sensor leads to a collision and thus needs to be prevented. The visual guided collision avoidance has been 


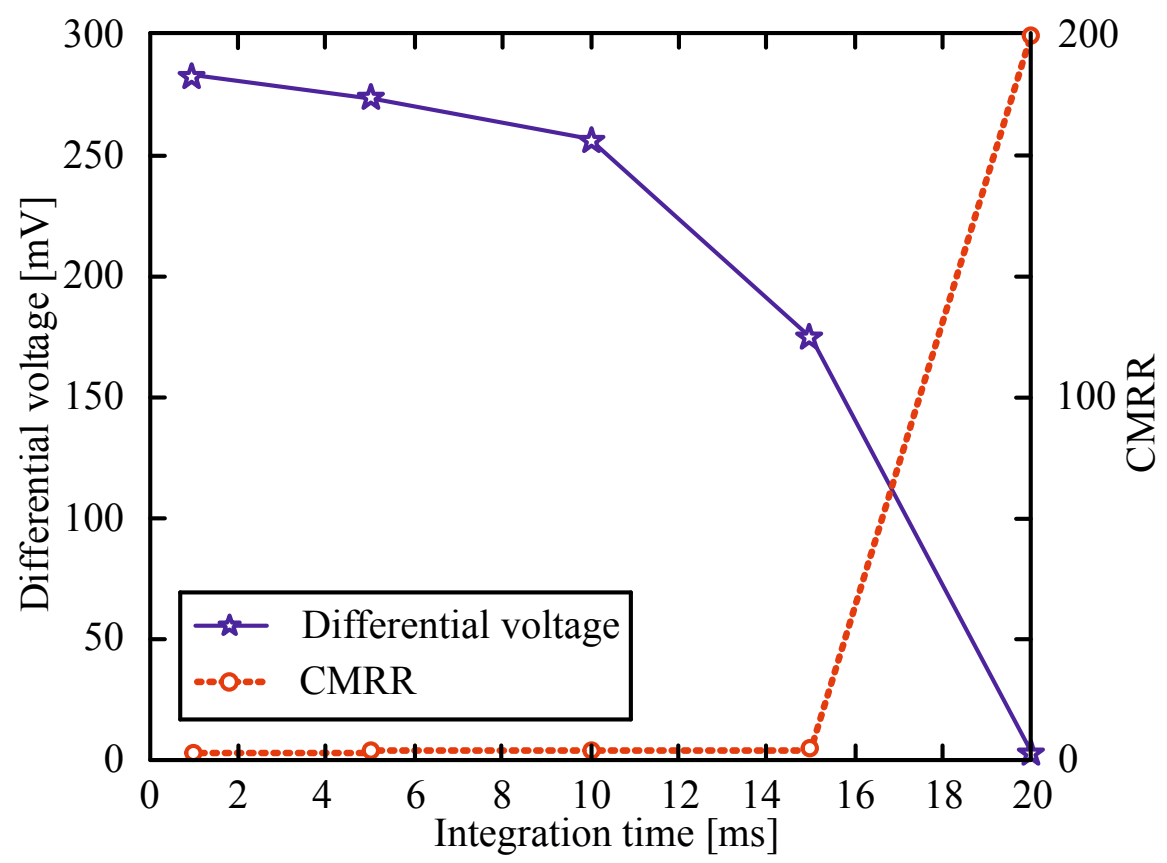

Figure 6.4: Variation in the differential swing and CMRR with changes in the exposure time.

extensively studied using conventional cameras and digital processing devices. However conventional cameras are not suitable for real time applications as they rely on motion estimation using sequential images.

It is well known that flying insects are able to detect obstacles in their flying path efficiently with little computational power by using optical flow. Optical flow is the pattern of apparent motion of objects, surfaces, and edges in a visual scene caused by the relative motion between an observer and the scene. Optical flow usually contains information about self motion and distance to potential obstacles and thus it is very useful for navigation [6.19]. In section 6.1, the three methods of obtaining optical flow for motion detection have been summarized. These methods are however broadly equivalent. In biological motion vision, the correlation based model or the gradient detector model are often used to account for the direction selectivity [6.20]. The correlation based model is shown to have significant advantages over the gradient based model in regimes where signal-tonoise ratio is low and detector noise is of concern.

The circuit of each pixel of the designed image sensor contains a comparator 
to detect the difference between the integrated charge from the photodiode and an external threshold voltage (chapter 2). This allows the generation of binary optical flow similar to the effect of "flickering" in the eyes of insects. The binary optic flow is the change in the digital pixel values in response to objects motion in the focal plane of the image sensor. The generated optical flow can be used to detect motion both in vertical (section 6.3.1) and horizontal (section 6.3.2) direction with minimal processing and hardware.

\subsubsection{Motion in vertical direction - collision detection}

In this section vertical motion in the focal plane is considered to estimate the time to collision of a moving object with the image sensor using binary optic flow. Reliable estimation of the time to collision between two moving objects is very important in many applications such as autonomous agent navigation or predictive crash sensors for autonomous safety systems. Currently existing non-biologically inspired collision avoidance systems use a CCD/CMOS camera and digital processing devices to detect the approaching object. Such a collision detection system is not suitable for compact real-time computations as it requires large amount of computations.

\subsubsection{Elementary motion detector (EMD)}

The most popular bio-inspired visual guided collision avoidance approach uses the correlation-type elementary motion detector $(E M D)$, first proposed by Hassenstein and Reichardt to compute the optical flow [6.21]. They have been used to explain directional selective motion in a wide variety of insects, birds and mammals including humans [6.10]. This model is very well established and often used in bio-inspired robots [6.22], [6.23] and [6.24]. The EMD correlates the response of one photoreceptor to the delayed response (inhibitory response) of an adjacent photoreceptor, both looking in the same direction as shown schematically in figure 6.5.

The elementary motion detector consists of two spatially separate inputs to measure the changes across space, temporal filters (delay) to measure the changes across time and a comparator to evaluate spatial and temporal changes. The coincidence of the original signal from one point in space and the delayed signal from the neighboring point in space leads to a positive output signal. The final output signal of the EMD is a transient response obtained by subtracting two images. The output image is sensitive to temporal changes in intensity of 


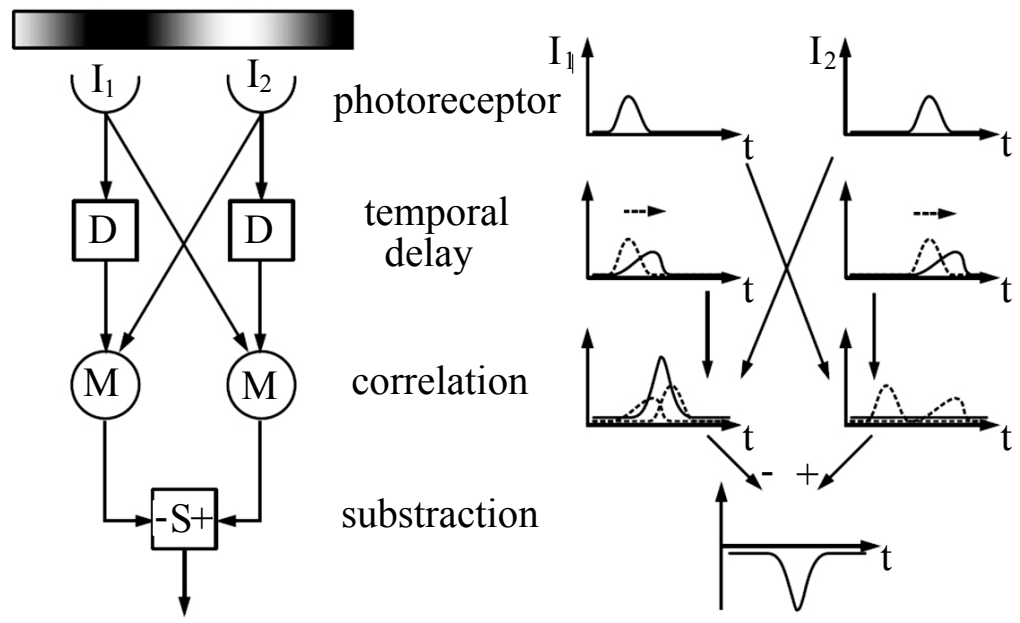

Figure 6.5: The Hassenstein-Reichardt or correlational elementary motion detector (EMD) [6.25].

light, enhancing the directional properties and motion detection by rejecting the effects of temporal contrast not resulting from motion. The motion detection by subtracting two images is better suited than the motion detection using derivative which amplify the noise.

The two separate inputs of the EMD model achieve what is referred to as "temporal decorrelation" or "transient enhancement" [6.23], which is a way of removing the redundant information from the photoreceptor signal before further processing to determine motion. The temporal decorrelation is a filtering mechanism, in which the DC light (mean ambient light) which doesn't have any motion information will be subtracted and AC light (which corresponds to motion) would be allowed to the next stage for further processing.

Existing implementations of the $E M D$ use a complex circuit with many active and passive components in order to obtain the inhibition of the signal and the correlation. Further because the basic collision detectors try to measure the velocity of the approaching object, they need dedicated processing blocks for velocity calculation from optical flow field. Additionally, the outputs of the $E M D$ are not invariant to the changes in the background illumination and their responses are not only proportional to velocity changes but are strongly affected by the contrast and the spatial frequency components of the scene [6.26]. Because the $E M D$ multiples its two input, it has quadratic dependence on the contrast and is thus not suitable for low contrast motions. This problem of quadratic 
dependence can be solved by introducing static expansive non-linearity in the channels [6.23] however this makes the model more complex. Further because the time delay in the two channels is constant, the EMD may infer a wrong conclusion if the time intervals of the moving object between the two points is not the same as the delay.

The standard output of a collision detector using an EMD is a response which stays low when the object is far enough and peaks before collision for an approaching object and then collapses to low values again [6.23]. The peaking of the collision detection algorithm depends on the delay in the delaying channel and the time an object takes to appear in the neighboring channel as well as the variations in the background illuminations [6.27]. Thresholding of this response to ascertain collision is thus difficult.

\subsubsection{Proposed EMD model for collision detection}

The correlation based models are usually computationally intensive and have difficulties in selecting reliable estimates. In order to have compact and lowpower real time autonomous motion detection systems, the estimation of the optical flow has to be simplified. This means that rough qualitative properties of the optical flow are more desirable for efficient collision detection than accurate target distance estimation [6.19]. The collision avoidance maneuvers in insects can be explained in terms of perception of looming stimuli or expanding images. The landing behavior of insects and the saccade (rapid turns) exhibited by example flies are believed to be triggered by image expansion as detected by an array of local motion detectors [6.28]. Collision detection using expanding images is also relatively independent of the spatial structure of the object being approached.

To understand the plenoptic function of expansion of images the motion constraint equation (6.9) is used. Since in collision detection the motion is assumed to be only in the vertical direction of the focal plane, the motion constraint equation can be rewritten as

$$
I_{y} v+I_{t}=0
$$

Equation (6.10) shows that the time of impact or time of collision of the object is directly proportional to the reciprocal of velocity of the object. Equation (6.10) can be better understood using figure 6.6, which shows an image of an approaching object of diameter $D$ at a constant velocity $V$ along the optical axis. The 
distance between the lens and the object is $d(t)$ while the focal length of the lens is $f$.

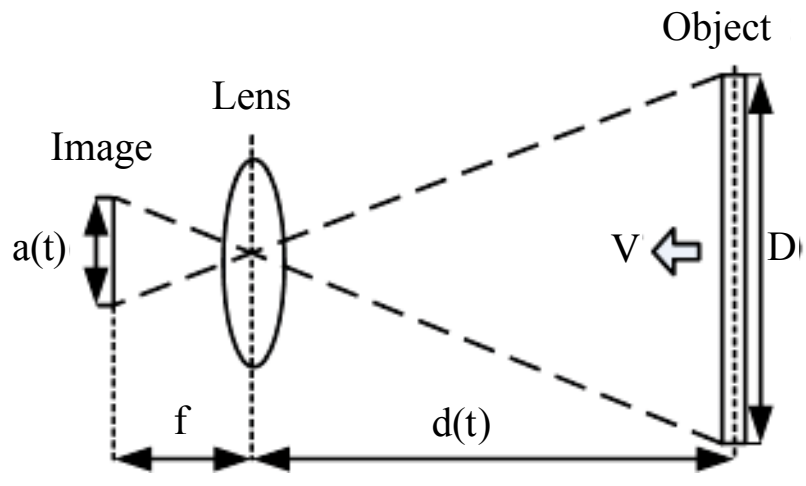

Figure 6.6: Perception of approaching objects.

The diameter of the obtained image and its derivative with respect to time is given as:

$$
\begin{aligned}
& a(t)=\frac{f \times D}{d(t)} \\
& \frac{d(a(t))}{d t}=-\frac{f \times D}{d(t)^{2}}
\end{aligned}
$$

The size of the image $a(t)$ in equation (6.11) increases with a decreasing distance $d(t)$ and vice versa. The change in the image size affects the optical flow perceived by the image sensor.

To simplify the optical flow generation algorithm, the binary output of the pixel is used. The binarization of the data in the pixel is explained in section 2.5.2.3. In the collision detection experiments, the image sensor is held stationary so that the optical flow is always generated by the motion of the object in the visual field. Figure 6.7 shows the variation in the light spot (approaching object). As the object moves closer to the image sensor, the image size (spot size on the imaging plane) grows or the optical flow expands. With the expanding of the optic flow the intensity profile of the pixel will also increase (figure 6.1(c)), and more pixels will have an output voltage higher than the reference voltage, and a digital ' 1 ' will be stored in the SRAM cells.

From the digital images, the percentage of active high pixels for a given illumination condition can be computed as the ratio of total active high pixel 


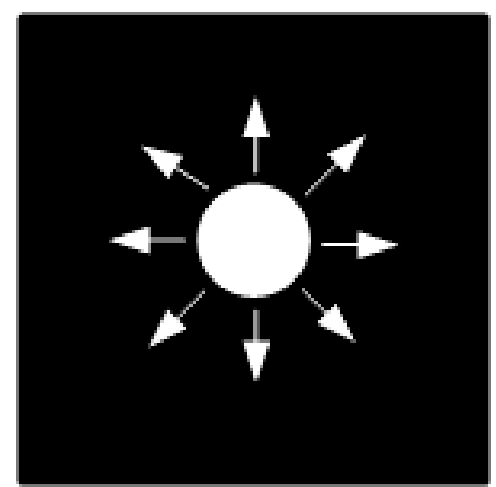

Figure 6.7: Perception of approaching objects.

to the total number of pixels in the array:

$$
\% \text { of active high pixels }=\frac{\text { Total active high pixels }}{\text { Total number of pixels }}
$$

Equation (6.13) represents the one-dimensional binary optical flow. The percentage of the active high pixels will increase with the approaching bright object as predicted by equation (6.11). The measured variations in the percentage of active high pixels with the variation in the distance of the light source for single image capture is shown in figure 6.8. It shows that when the light source approaches the image sensor, the optical flow, which is the variation in the intensity with motion, causes more pixels to become active thus increasing the percentage of active high pixels.

One of the major requirements of motion detection using correlation models is temporal decorrelation. Temporal decorrelation refers to the process of reducing the autocorrelation with a signal in time domain, this helps in removing redundant information in images separated in time. Temporal decorrelation can be obtained using differential imaging, where two samples are spaced in time. The differential image is generated using partial charge transfer, where the integrated charge at the photodiode capacitance is transferred to the $F D$ node multiple times in one frame (chapter 2). Figure 6.9 shows the algorithm used.

The first image capture is at a time instance of $T_{1}$ and the second capture is at $T_{F T}$. The captured samples are then compared with the reference voltage and the digital output is stored in the two SRAM cells available in the pixel. The differential image of the two spatially integrated digital images obtained from SRAM1 and SRAM2 was computed off chip for this version of the sensor. 


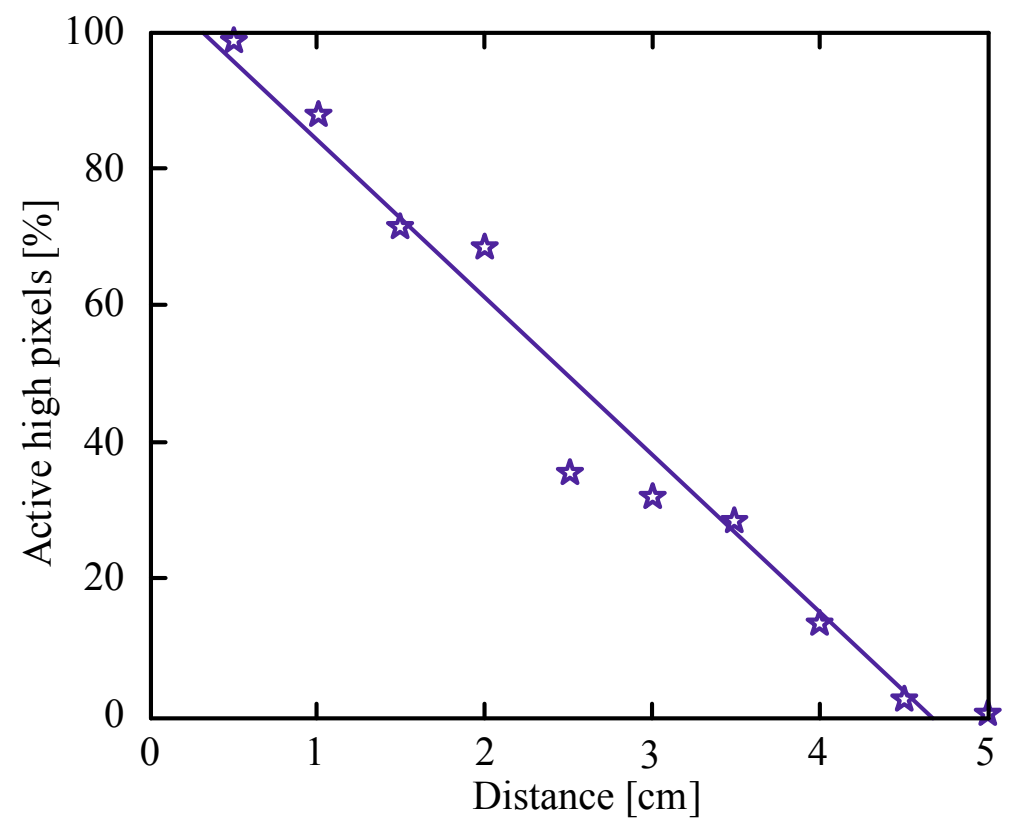

Figure 6.8: One-dimensional binary optical flow variation with approaching object.

The temporal decorrelation of the optic flow obtained using the partial charge transfer is shown in figure 6.10. The figure shows the variation in the percentage of active high pixels of two image captures for varying distance of the object from the imager. The first image is captured after an integration time of $T_{1}$ and the second after the total frame time $T_{F T}$. Two measurements for $T_{1}$ when $10 \mathrm{~ms}$ and $1.5 \mathrm{~s}$ are shown in the figure 6.10 . The variation of the time instances allows to generate varying decorrelated one-dimensional binary optical flow.

The difference of the two temporally decorrelated optical flows is plotted in the figure 6.11. It can be observed that as the object moves towards the image sensor, it has a certain threshold of percentage of pixels with changed states below which the object is very near to collision. In this case the collision detection mechanism does not need to use dedicated motion processing blocks. The collision can be detected to a very good degree of reliability using the percentage of changed pixels with the varying one-dimensional differential optical flow.

Harrison [6.23] uses a collision detection algorithm based on the basic EMD model and the algorithm peaks at $230 \mathrm{~ms}$ before collision for an object with a velocity of $17 \mathrm{~cm} / \mathrm{s}$. Thus the collision alert is generated at a distance of 


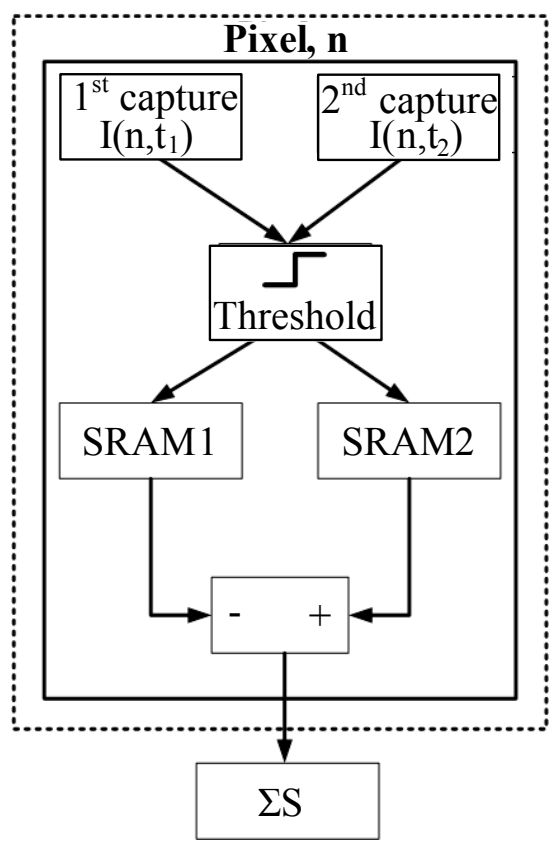

Figure 6.9: Modified EMD model for collision detection.

approximately $4 \mathrm{~cm}$. A similar method used by Okuno and Yagi [6.22] produces a collision detection alert at a distance of $63 \mathrm{~m}$. The differential optical flow is shown to generate a collision alert for distances less than $2 \mathrm{~cm}$. For narrow path autonomous agent navigations, a collision alert for very small distance is desired. For example in applications like endoscopy, a collision alert distance of less than a $\mathrm{cm}$ would be ideal. The distance of collision alert can also be modulated by varying the $T_{1}$ and $T_{F T}$, depending on the application and situation.

\begin{tabular}{|c|c|c|c|}
\hline Method used & $\begin{array}{c}\text { EMD model based } \\
\text { on locust }\end{array}$ & $\begin{array}{c}\text { Delay and } \\
\text { correlate } E M D\end{array}$ & $\begin{array}{c}\text { Differential optical } \\
\text { flow imaging }\end{array}$ \\
\hline $\begin{array}{c}\text { Collision alert } \\
\text { distance }\end{array}$ & $63 \mathrm{~m}$ & $\sim 4 \mathrm{~cm}$ & $\leq 2 \mathrm{~cm}$ \\
\hline
\end{tabular}

Table 6.1: Performance comparison.

Furthermore the proposed algorithm doesn't suffer from the inherent disadvantage of the EMD model in the accurate detection of the output peak. The output of the differential optical flow continues to stay low near collision 


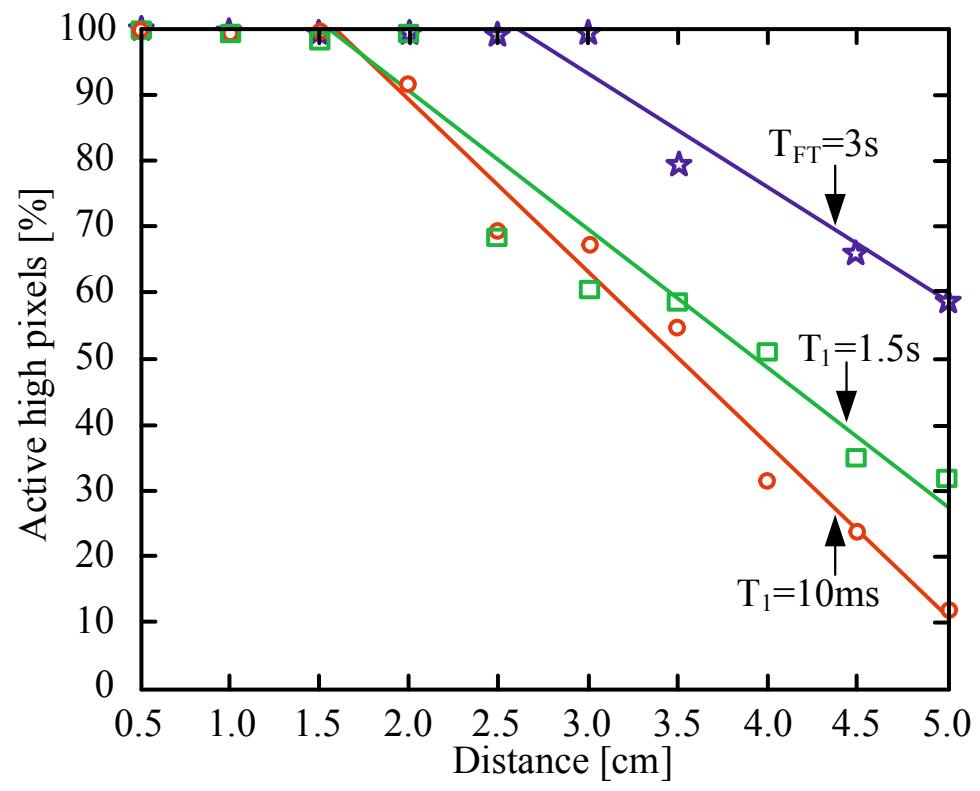

Figure 6.10: Temporally decorrelated optical flow with approaching objects.

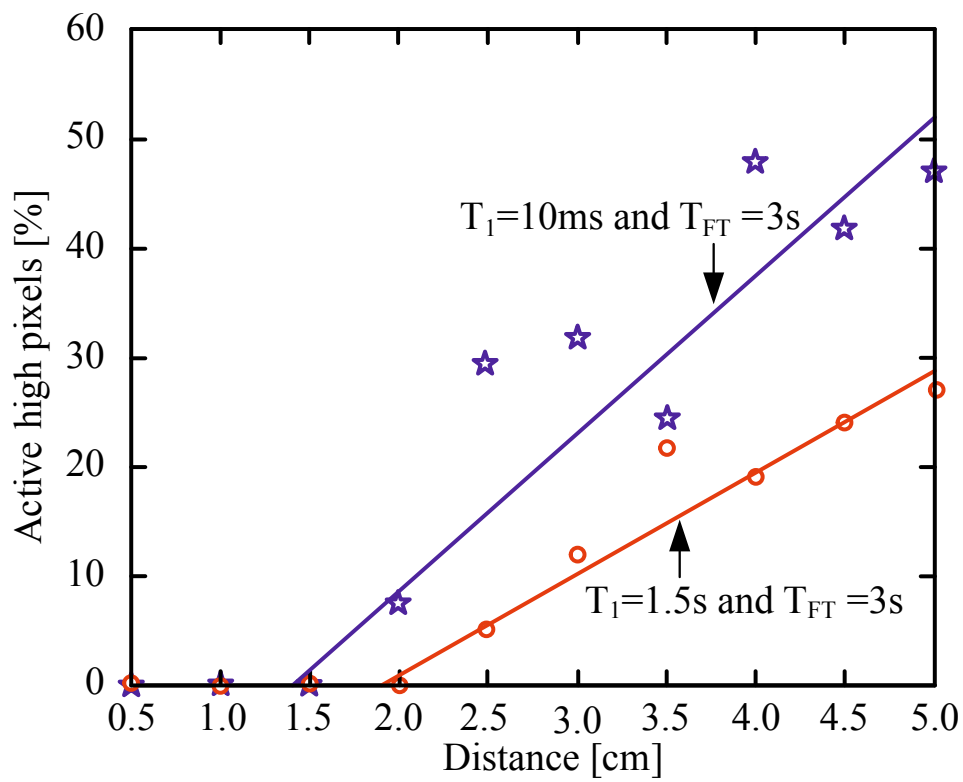

Figure 6.11: Temporal differential decorrealated optical flow with approaching object. 
allowing thresholding and thus is more stable. By modulating the differential time, it would be possible to prevent collision in very narrow paths thus helping navigation of the autonomous agents. Further as most of the computations are done on focal plane there is no image transfer bottleneck, which is usually present in the conventional approach of using an image sensor together with a digital signal processor.

So far the collision detection algorithm is employed in an environment with an illuminated object moving towards the image sensor in a dark background. However since the temporally decorrelated signal is a differential signal, it would also work in an environment condition where a dark object moves vertically with respect to the image sensor in a light background. In such a case initially the binary optic flow would be composed off all digital ' 1 ' and as the dark object approaches the image sensor, the optic flow would toggle to digital ' 0 '. The temporally decorelated difference image would thus subtract two images whose optic flow would be dominated by digital ' 0 '. The difference image would thus show the $\%$ percentage of active high pixels to exhibit a behavior similar to figure 6.11 .

\subsubsection{Motion in horizontal direction}

In this section only horizontal motion is considered. From the pixel array only the binary output of the comparators are used for the motion detection. The 7bit counter counts the number of ' 1 ' 'in each row of the pixel for each frame. The algorithm for motion detection then compares the counter outputs to decide if there is motion. If the difference of the counter outputs for two exposures is higher than a certain threshold, motion occurrence is flagged.

For the designed sensor the brightness control voltage is the reference voltage to which the analog signal obtained after each exposure is compared. The two SRAM cells in the pixels serve as frame latches and offer both past and current data. The pixel converts the image data into a one-bit data stream by the comparators.

To verify the proposed model two consecutive frames of a light source moving over the image sensor are shown in figure 6.12.

The left image shows the light source at its initial position and the right image shows it after a slight movement. The two images look very similar, as only a very small motion was introduced. The histograms of the two images are shown in figure 6.12. The subtraction of the two images results in a difference image, and the histogram of the pixels which changed states are shown at the bottom of 


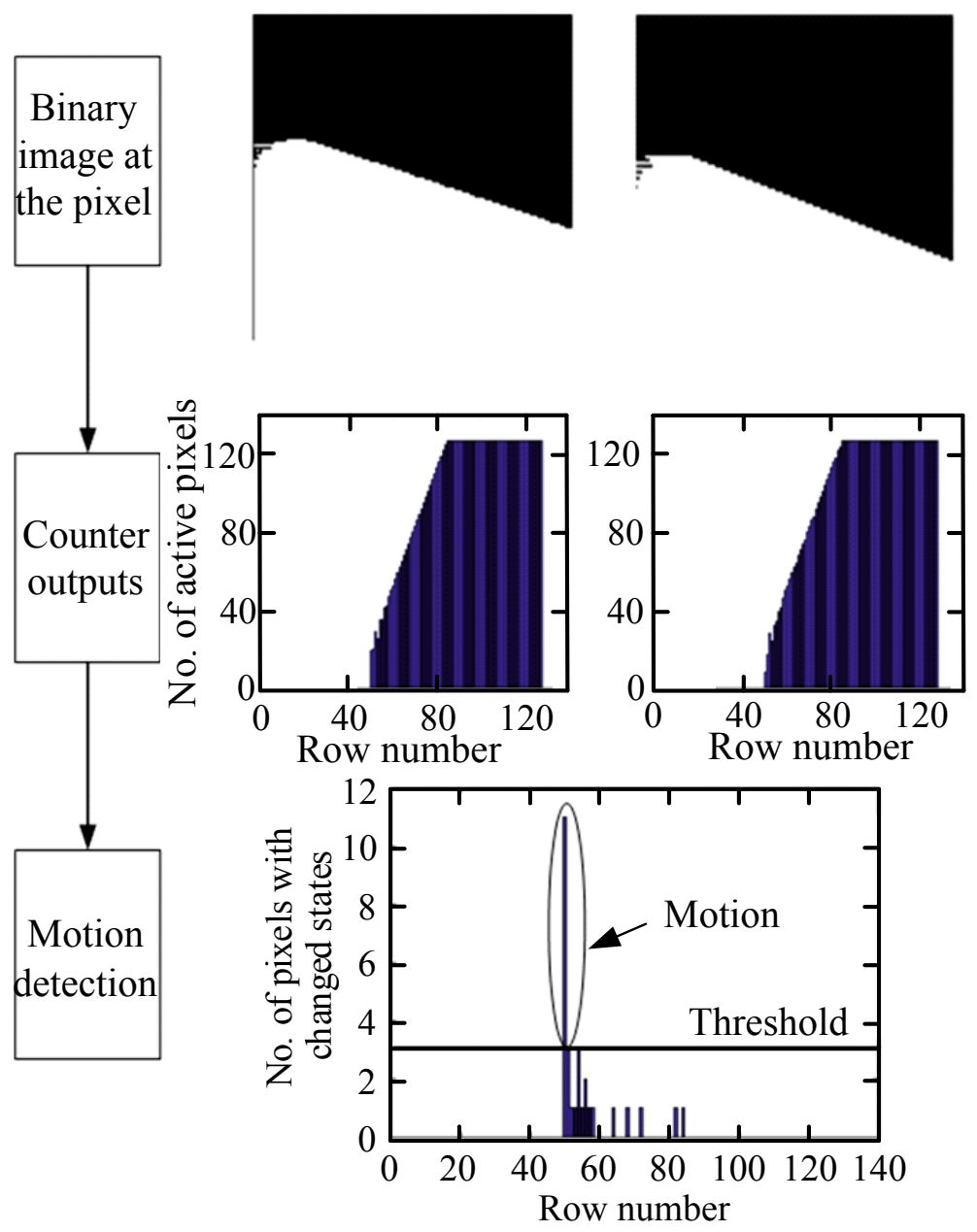

Figure 6.12: Horizontal motion detection using spatially integrated binary optical flow.

the figure. By selecting a proper threshold, accurate detection of motion can be done. 


\subsection{Illumination invariant and high dynamic range motion detection}

The outputs of the EMD are not invariant to the changes in the background illuminations as discussed in section 6.3. This problem is illustrated in figure 6.13 which shows the increase in the $E M D$ output with the increase in the background illumination.
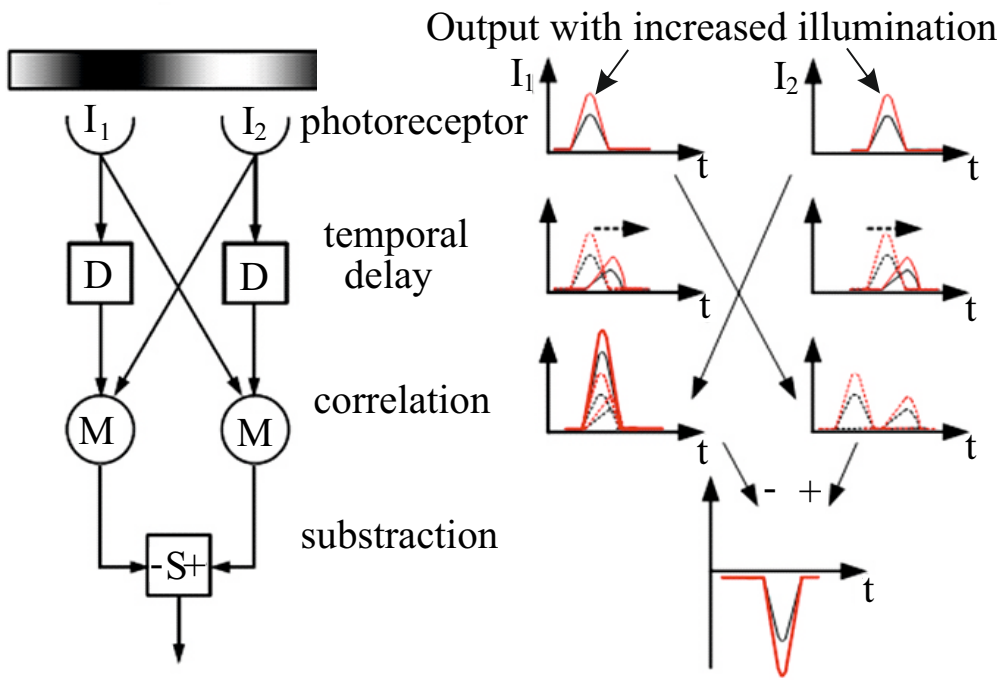

Figure 6.13: The correlational elementary motion detector showing increased motion for change in illumination.

An increase in the background illumination increases the output of the EMD and this increased output can be confused with motion. Also it is shown than the optical flow computed on the image doesn't match actual motion with non-uniform illumination [6.29]. The estimation of motion parameters from the spatiotemporal patterns of visual stimuli or optical flow implicitly requires responses that are invariant with respect to absolute illumination levels, contrast and the spatial structure of the scene.

For the $E M D$ model to have a good performance over varying illumination conditions, the dynamic range of the sensor also needs to be high. A real-world scene is composed of varying level of brightness within it. The dynamic range of the scene is typically always higher than the dynamic range of the sensor used to capture the scene. A typical image sensor has a dynamic range of about 65 to $75 \mathrm{~dB}$ while a scene can have a dynamic range of over $120 \mathrm{~dB}$. In high illumination 
conditions the photodiode of the pixel saturates very quickly. For a very bright object most of the photodiodes will be saturated and thus finer details and motion cannot be captured.

To increase the dynamic range either the maximum signal at the photodiode node (well capacity) has to be increased or the read noise has to be decreased(equation (2.11)). The relationship between output, integration time and photocurrent at the floating diffusion node of a linear image sensor is given by:

$$
V_{\text {signal }}=\frac{t_{\text {int }} \times I_{\text {photo }}}{C_{\text {eff }}}
$$

The photocurrent produced in the photodiode is proportional to the irradiance and the light collection area or the photodiode area. The larger the pixel exposed surface, the more light it can collect during the exposure period and thus the higher is the dynamic range. The photodiode area is limited by pixel size constraints. For high resolution a small pixel size is desired. This leaves us with two parameters in equation (6.14) that can be modified to increase the dynamic range: the integration time $t_{i n t}$ and the effective capacitance to store the collected charges during integration $C_{e f f}$. The total capacitance $C_{\text {eff }}$ on the photodiode sense node is the sum of the junction capacitance proportional to the diode area and the parasitics due to the sensing and amplifying circuitry.

From equation (6.14) it is clear that the slope of the pixel response can be changed by the scaling of the effective capacitance. Changing the effective capacitance varies the well capacity. The effective capacitance can be varied using either smart reset pixels [6.30], using overflow MOSFET capacitors [6.31] or by using multiple shorter exposure periods [6.32], [6.33]. In the smart reset pixels, the reset gate voltage is monotonically decreased during integration causing the well capacity (charge capacity) of the sensor to monotonically increase. Using a lateral overflow capacitor, the overflowed charges from a fully depleted photodiode during an exposure can still be integrated. The disadvantage of such a scheme is the effective increase of the floating node capacitance which reduces the charge conversion gain and also the fill factor of the pixel. In the multiple shorter integration period methods, several images with different exposure time are captured. The images with a shorter exposure time captures the brightest areas of the scene, while the images with longer exposure time capture the darker areas of the scene. A high dynamic range image is then synthesized from the multiple captures. 
Since differential imaging is used in the proposed model of the EMD to obtain the temporal decorrelation necessary for motion detection, a partial charge transfer method is used to increase the dynamic range of the model. This method provides a signal after a short integration time in addition to a signal after a long integration time [6.18]. The difference of the two charge accumulation times in one frame extends the dynamic range of the sensor by removing the static or DC light.

The photo-conversion characteristics for the complete charge transfer (DDS) and the differential signal for the partial charge transfer with various integration is shown in figure 6.14. $T_{1}$ is varied from $5 \mathrm{~ms}$ to $20 \mathrm{~ms}$.

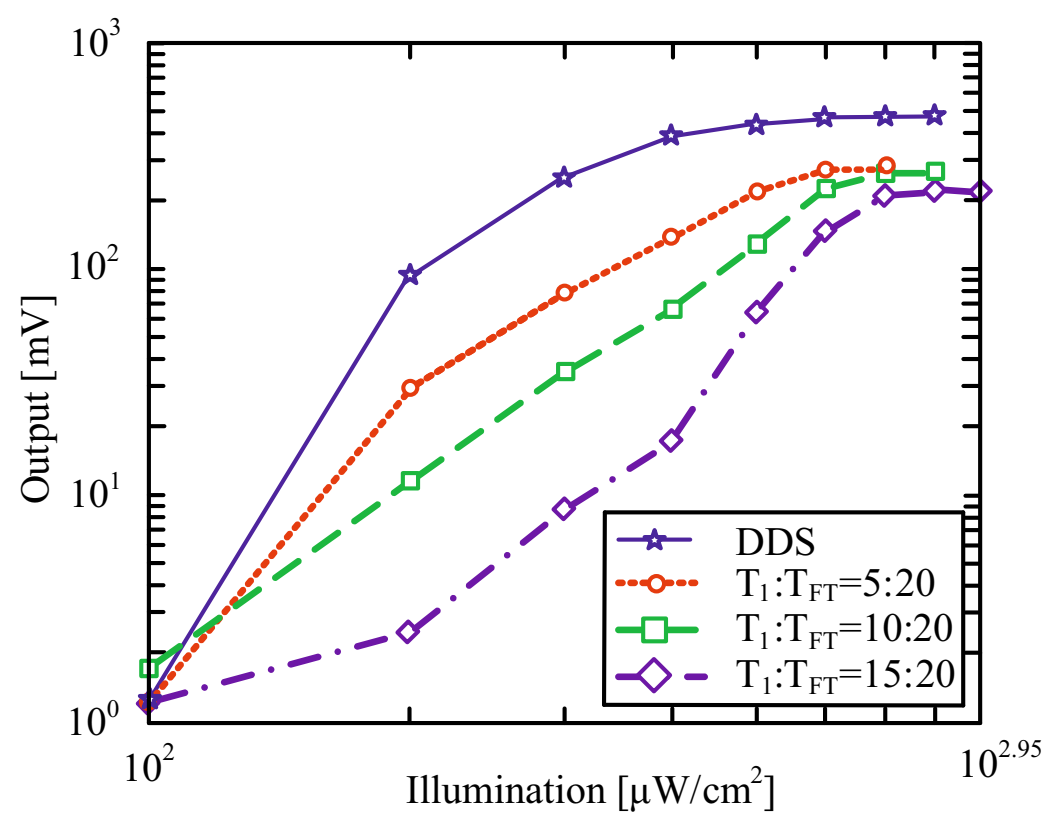

Figure 6.14: Photo-conversion characteristics at $\lambda=550 \mathrm{~nm}$.

Figure 6.15 shows the variation in the saturation intensity with varying $T_{1}$ : $T_{F T}$ ratio. The saturation intensity is the intensity of light causing the photodiode to saturate. It is observed that the saturation intensity increases with an increase in $T_{1}: T_{F T}$ ratio or with an increase in the accumulation time $T_{1}$. By appropriately changing the $T_{1}: T_{F T}$ ratio, the saturation intensity can be doubled thus increasing the sensitivity to the background brightness.

Figure 6.16 shows an example of increasing the saturation level using partial charge transfer. Images of a DC light source were taken with different $T_{1}: T_{F T}$ 


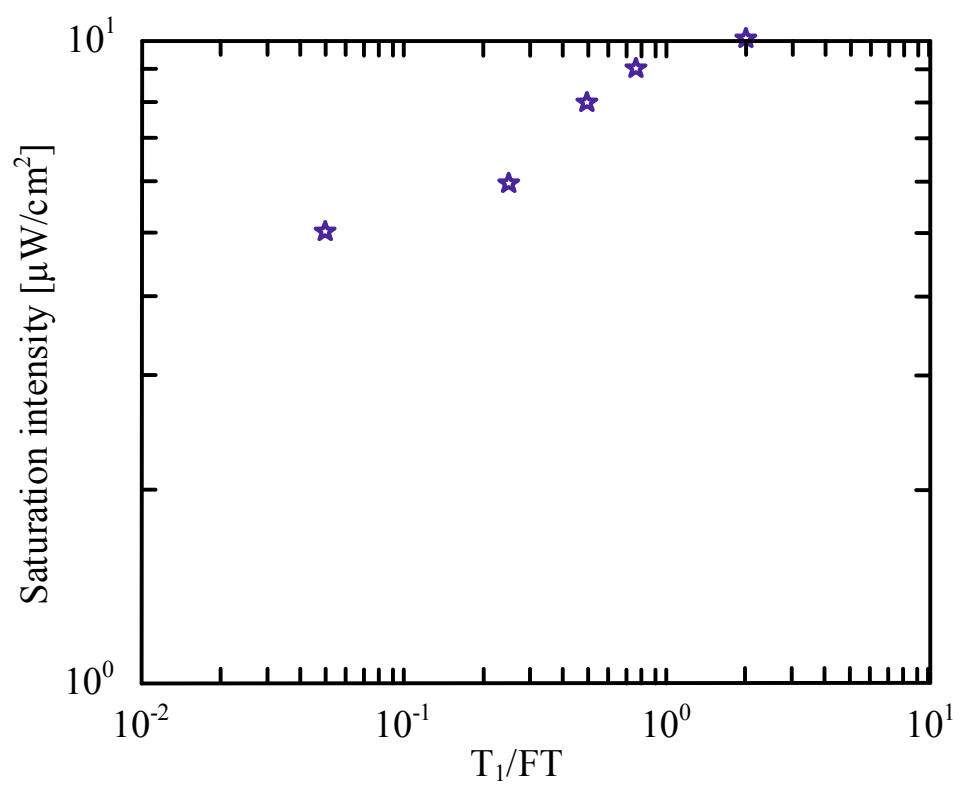

Figure 6.15: Saturation intensity variation with variation in $T_{1}: T_{F T}$ ratio.

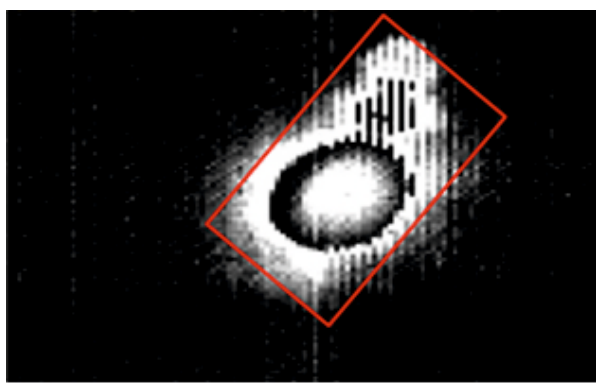

(a)

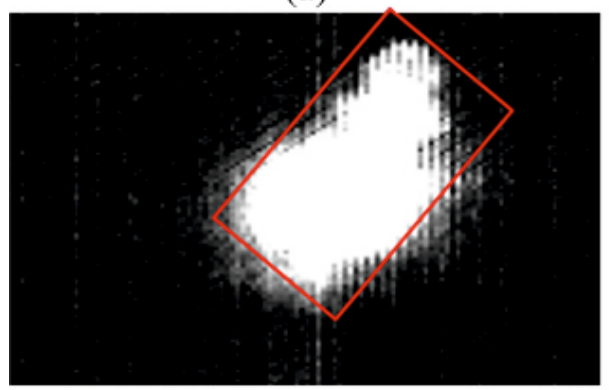

(b)

Figure 6.16: DC light source images with $T_{1}: T_{F T}$ of $(a) 0.0005$ and $(b) 0.05$. 
ratios. Figure 6.16(a) and (b) show the images with $T_{1}: T_{F T}$ values of 0.0005 and 0.05 respectively. In figure 6.16(a) the pixels at the bright portions of the DC light source saturate, causing an overflow with a very short accumulation time $T_{1}$. The differential signal with increased $T_{1}$ is shown in figure 6.16(b) and is able to capture the bright portion of the DC light source.

In partial charge transfer, when the charges are transferred from the photodiode to the sense node, some amount of charge is left in the photodiode, as shown in figure 2.18(d) (chapter 2). This introduces image lag and can be removed by resetting the photodiode. However, a reset introduces $k T / C$ noise. The amount of residual charge in the photodiode depends on the accumulated charge. Since the accumulated charge is proportional to the exposure time, the residual charge increases with the exposure time. The increase in the residual charge increases in turn the non-linearity of the photo-conversion characteristic and further decreases the sensitivity. The accumulated signal obtained after partial charge transfer with a shorter $\left(T_{1}\right)$ period is also non-linear with respect to the incident light. The nonlinearity is believed to be caused by the carrier diffusion and the initial condition of the photodiode [6.18].

The increase in non-linearity at higher illumination levels for the partial charge transfer can be modeled using figure 6.17. It shows the accumulated charges for various intensities of light $I_{t}$ (normal), $I_{\max }$ (maximum) and $I_{\min }$ (minimum) and varying $T_{1}\left(T_{11}, T_{12}, T_{13}\right.$ and $\left.T_{14}\right)$.

$N_{F T}$ is the number of electrons accumulated when the sensor is operated in conventional or complete charge transfer mode with a frame time of $T_{F T}$. For the partial charge transfer mode $T_{1}$ can vary and four different time instances are shown in figure 6.17. For illumination $I_{t}$, the following equations hold

$$
q \times N_{T 12}=I_{t} \times T_{12}
$$

If $N_{a 1}$ is the number of residual charges after the first charge transfer at $T_{12}$ for a illumination of $I_{t}$ then

$$
\begin{aligned}
& q \times\left(N_{F T}-N_{a 1}\right)=I_{t} \times T_{F T} \\
& N_{T 12}=\frac{T_{12}}{T_{F T}} \times\left(N_{F T}-N_{a 1}\right)
\end{aligned}
$$

For the highest illumination $I_{\max }$, the photodiode saturates very quickly. $N_{a 2}$ is the residual charge after the first charge transfer at $T_{12}$ for an illumination of $I_{\max }$ 


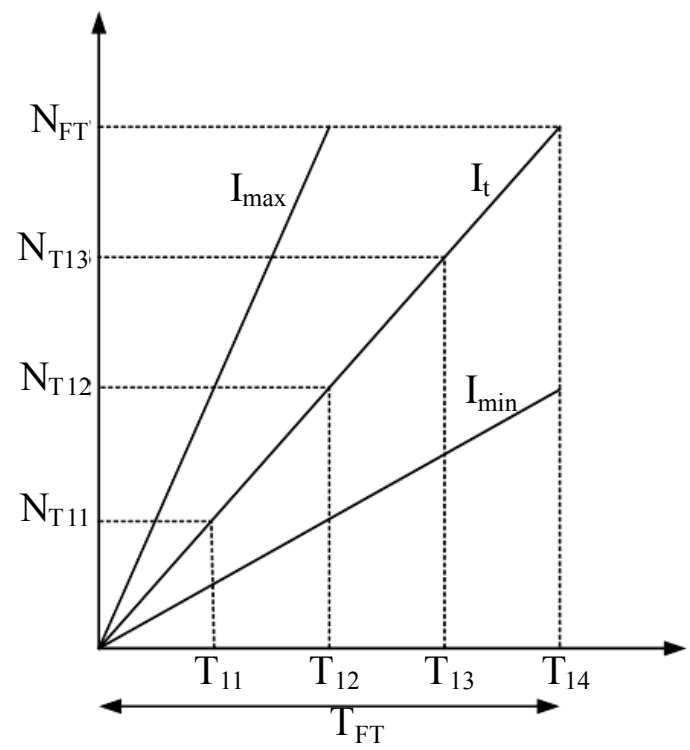

Figure 6.17: Charge accumulation for various illumination and integration periods.

$$
\begin{aligned}
& q \times N_{T 12}=I_{\max } \times T_{11} \\
& q \times\left(N_{F T}-N_{a 2}\right)=I_{\text {max }} \times T_{12} \\
& N_{F T}^{2}-a_{1} \times N_{F T}+b_{1}=0
\end{aligned}
$$

where

$$
\begin{aligned}
& a_{1}=N_{a 1}+N_{a 2} \\
& b_{1}=N_{a 1} \times N_{a 2}-\frac{T_{F T}}{T_{11}} N_{T 12}^{2}
\end{aligned}
$$

Figure 6.18 shows the photo-conversion characteristics of the synthesized wide dynamic range signals with the dynamic range extension ratio $T_{1}$ to $T_{F T}$ set to 1:20, 5:20 and 10:20 for higher illuminations. It is observed that for decreasing $T_{1}$ to $T_{F T}$ ratios the non-linearity increases at high illumination. It is further observed that with the decrease in the $T_{1}$ to $T_{F T}$ ratio the sensitivity increases.

The sensitivities obtained for $T_{1}: T_{F T}$ ratio of 10:20, 5:20 and 1:20 are 11.46 


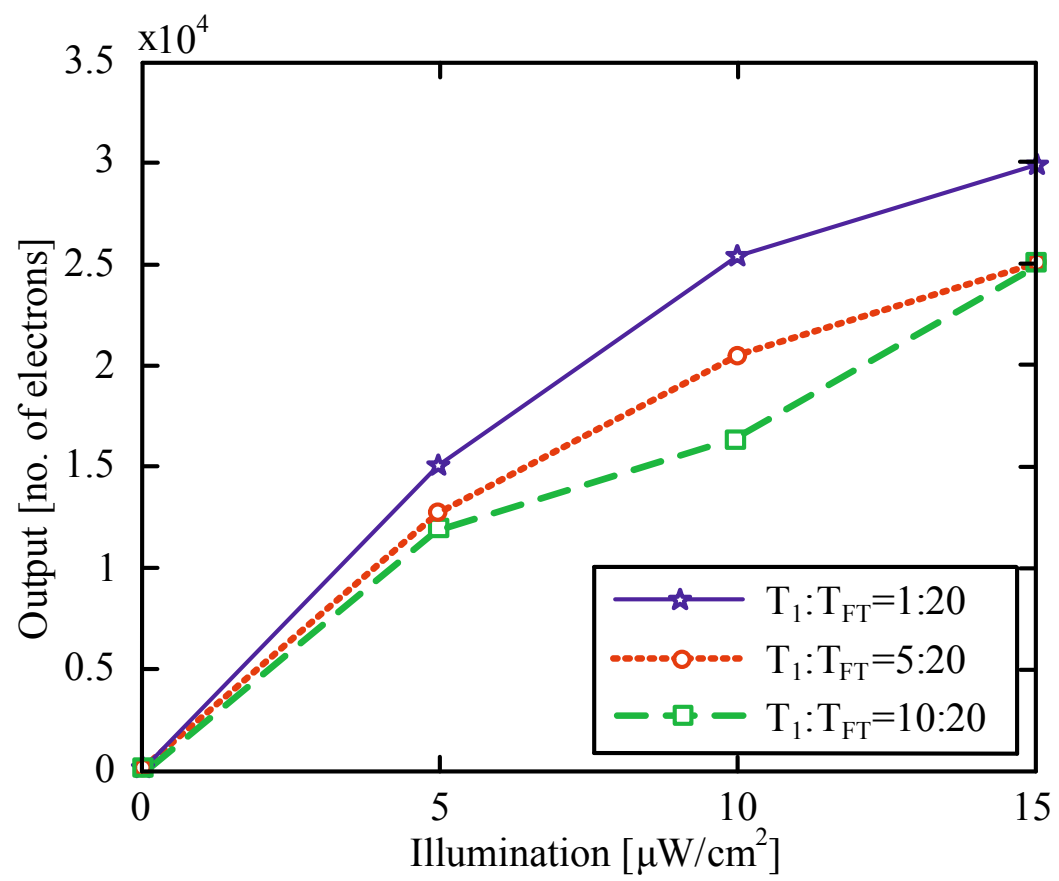

Figure 6.18: Non-linearity at high illumination for partial charge transfer.

$\mathrm{mV} / \mathrm{ms}, 12.89 \mathrm{mV} / \mathrm{ms}$ and $14.37 \mathrm{mV} / \mathrm{ms}$ respectively.

The associated non-linearity at high illumination with partial charge transfer is interesting, as in motion detection a non-linear interaction between two spatially separated signals is usually desired [6.34]. In insects the photoreceptors adapt to the mean luminance in the enviornment and give an approximately logarithmic response to changes in light intensity [6.35]. This compressive non-linearity allows a better response to contrast than absolute luminance. Non-linearity in the Hassenstein-Reichardt EMD model is introduced either by thresholding or rectification to obtain a saturating contrast response curve for motion adaptation [6.36], [6.37]. The effect of the non-linearities associated with high illumination obtained with partial charge transfer on motion detection is yet to be explored.

The collision detection algorithm in section 6.3 is designed using onedimensional binary optical flow. Figure 6.19 shows the percentage of active high pixels when the photodiode is operated in complete charge transfer $\left(T_{1}=0 \mathrm{~ms}\right)$ and partial charge transfer $\left(T_{1}=10 \mathrm{~ms}\right)$ respectively. It is observed that with partial charge transfer higher illumination levels are captured, thus increasing the digital dynamic range of the image sensor. 


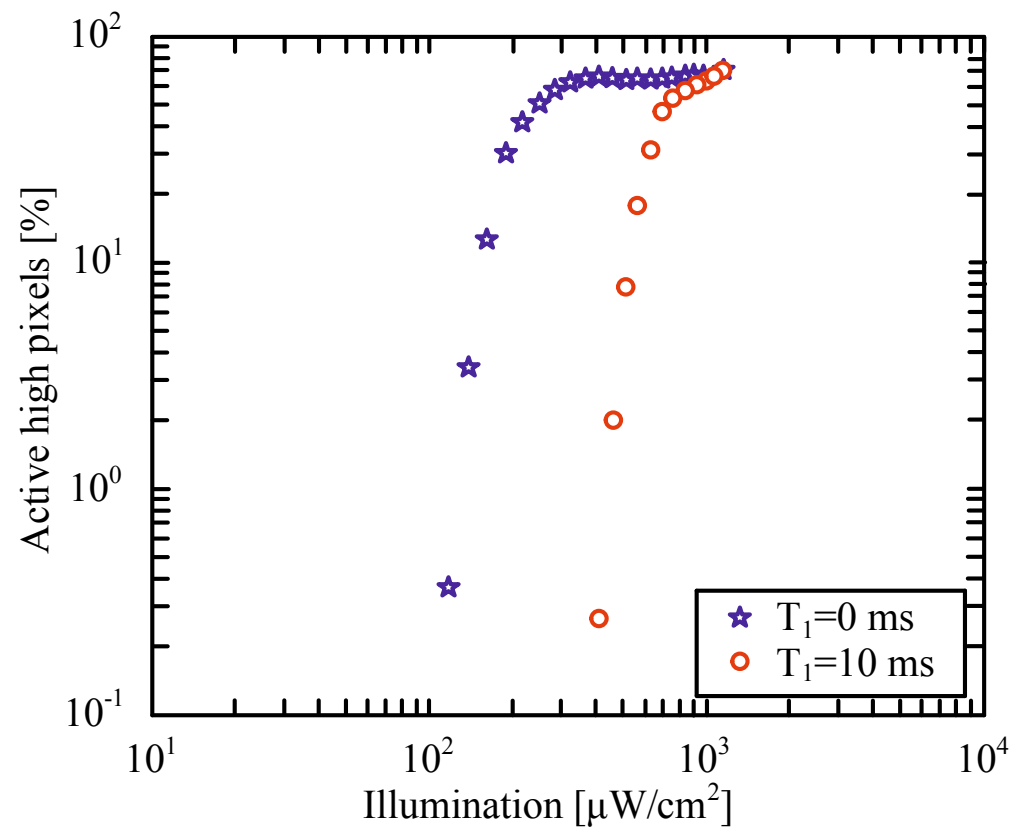

Figure 6.19: Variations in the optical flow for partial charge transfer.

\subsection{Digital polarization}

In chapter 3 a CMOS polarization sensor with embedded metallic wire gird was discussed. The image sensor was shown to respond to the changes in the polarization angle. This polarization sensitivity was further shown to be useful in machine vision applications like material classification and autonomous agent navigation in chapter 4 and 5 respectively. However the proposed solutions for these applications were based on using the analog signals from the pixels. In section 6.3, a simple way to calculate one-dimensional-correlational optical flow using the number of active high pixels at a given time was introduced. The number of active high pixels will vary depending on the horizontal and vertical motion of the object source and its relative intensity variations on the focal plane. Here only motion in vertical direction to the imaging plane is considered. Thus depending on the object moving close or far away from the image sensor, the spot on the focal plane increases or decreases. The size of the spot will be further dependent on the polarization angle of the incoming light ray and the one-dimensional binary optical flow can thus be used to represent polarization in digital format. 
The expected theoretical behaviour of the one-dimensional binary optical flow for the two polarization sense regions (chapter 3) is shown in figure 6.20.

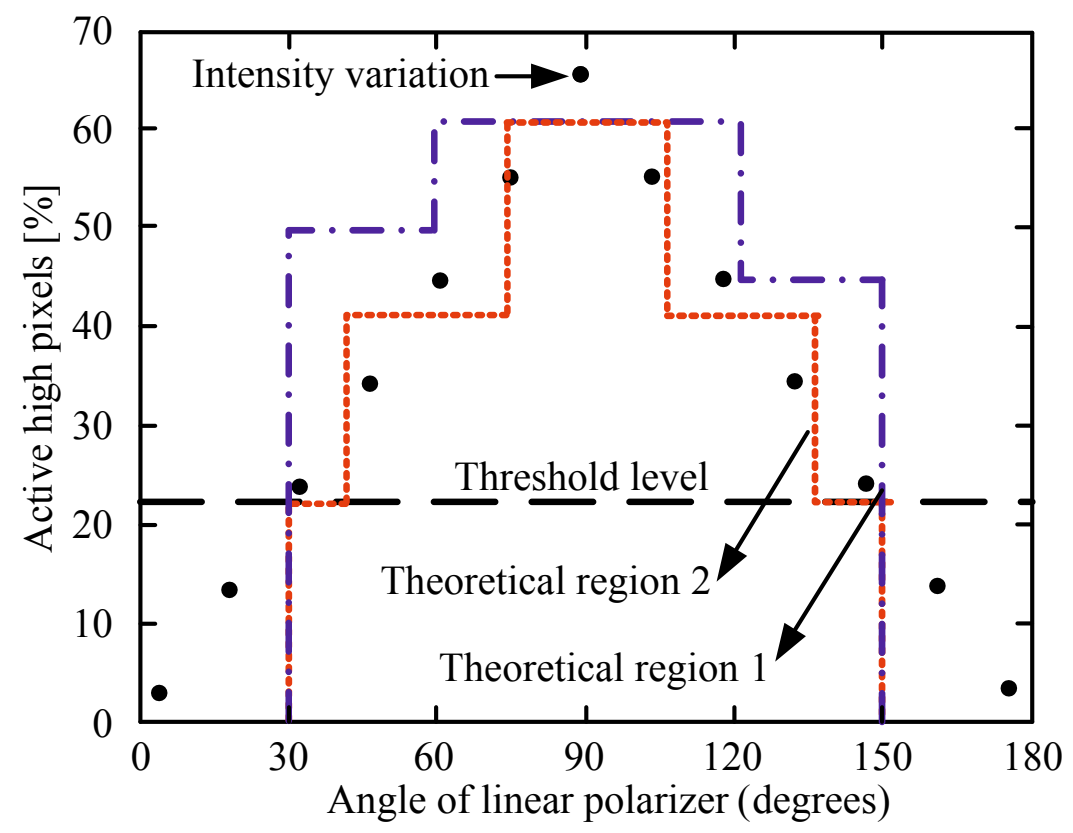

Figure 6.20: Theoretical behaviour of one-dimensional optical flow with variation in linear polarizer angle.

For the polarization sense region 1 , when the object is moving towards the imaging plane the intensity of the pixels slowly increases. As the light intensity reaches the threshold level, one expects the two intensity sensitive pixels to store a digital '1', the output of the comparator, in the SRAM cells. The percentage of active high pixels shows a step rise to around $50 \%$. As the intensity is further increased around $90^{\circ}$, the $90^{\circ}$ sensitive pixels will slowly start to have a have a digital ' 1 ', as their comparators' outputs, and hence the percentage of active high pixels will increase above 50\%. Ideally, when all the $90^{\circ}$ sensitive pixels turn high, $75 \%$ of the pixels in region 1 will be active high. However due to the attenuation of the light by the external polarizer not all $90^{\circ}$ sensitive pixels turn high. In the polarization sense region 2 , the theoretical behavior of the percentage of active high pixels would be the same as region 1, except that as the intensity increases, the $45^{\circ}$ sensitive pixels start to turn high. Thus there is an additional step rise when the polarizer angle is $45^{\circ}$. In the experiments, the optical flow is obtained by increasing the light spot gradually from the center to the periphery 
of the polarization sense regions, using a linear polarizer. This results in a linear increase in the percentage of active high pixels with the variations in the linear polarizer angle, instead of the expected step rise.

The experimental setup for the measurement is the same as described in chapter 3 for analog polarization measurements. The analog performance for the $90^{\circ}$ polarizer filter in the two polarization sense regions 1 and 2 is compared with the one-dimensional binary optical flow for varying angle of linear polarizer in figure 6.21.

The measured one-dimensional binary optical flow is shown to have an angular dependence on the angle of the linear polarizer and is very similar to the theoretically predicted behavior. The optical flow and analog representations of polarization in region 2 match closely. It can be predicted that by increasing the number of metallic wire grid orientations over the photodiode a digital representation of polarization very similar to the analog representation can be obtained [6.38].

A generalized algorithm to represent polarization information will have multiple advantages in low level polarization based machine vision applications. Based on the one-dimensional binary optical flow variations with the polarization angle, a way to determine the Stokes parameters, degree of polarization and polarization Fresnel ratio in binary format can be formulated which will allow focal plane processing of applications like material classification and autonomous agent navigation. Such a sensor would be miniaturized, bandwidth compressor and low power which are highly desirable in the future generations of sensors in machine vision applications.

\subsection{Conclusions}

The compound eyes of insects are better suited for detection of motion. Inspired by them a CMOS image senor is designed operating in temporal differential mode and spatial integration of one-dimensional binary optical flow to detect motion/collision of moving objects. The binary optical flow is generated in-pixel from multiple images stored in the in-pixel memories and spatially integrated using a counter. The computations are relatively easy and experimental results show the ability to detect collision with the approaching object as near as $2 \mathrm{~cm}$. This method allows the design of simple, miniaturized, low power and narrow path autonomous navigating agents. The dynamic range of a sensor can be enhanced using partial charge transfer, which also provides for a background illumination invariant motion detection system. 


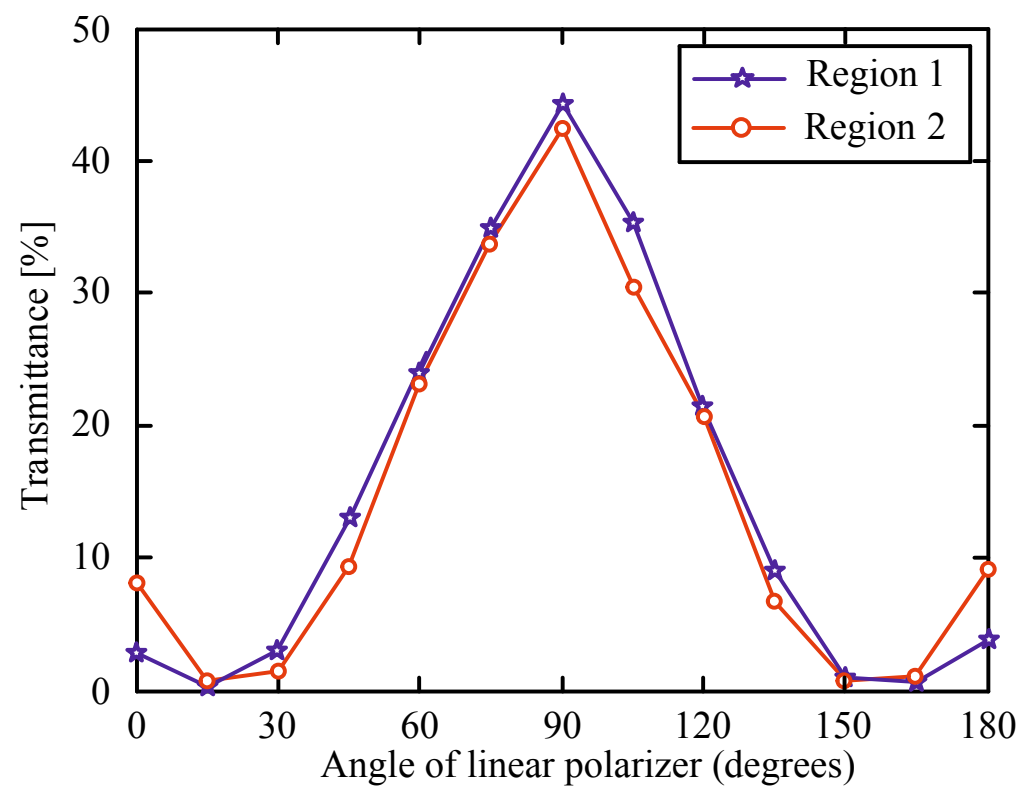

(a)

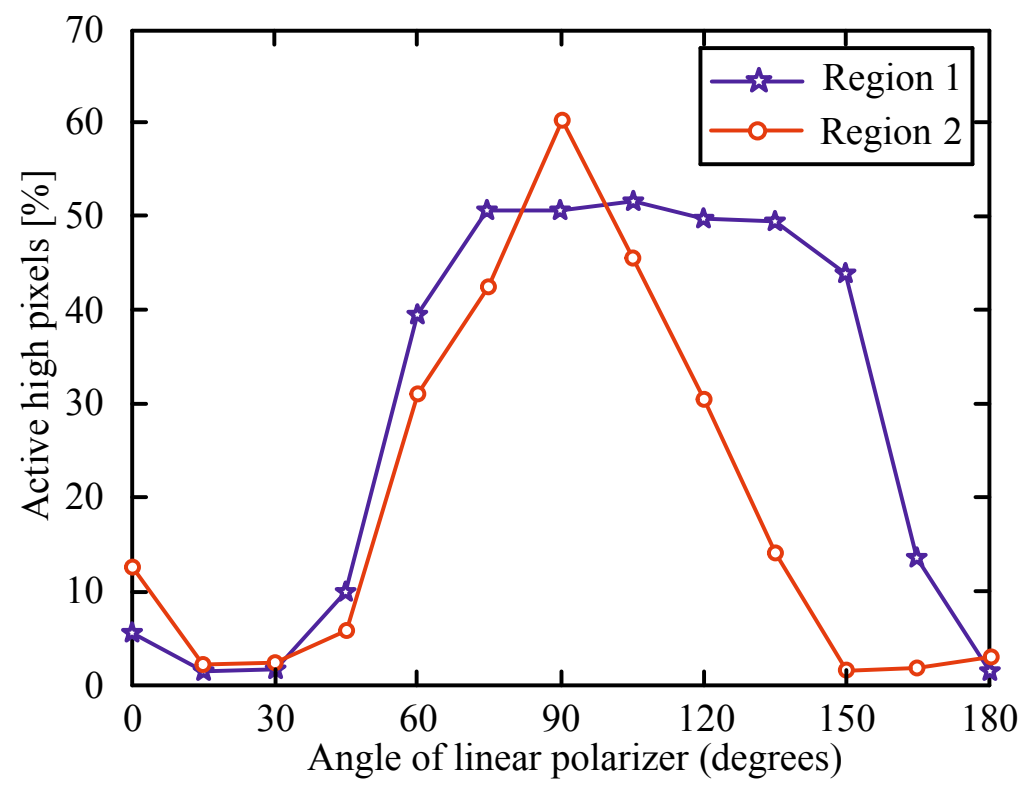

(b)

Figure 6.21: $90^{\circ}$ polarization in sense regions 1 and 2: (a) analog (b) digital (optical flow variation). 
The one-dimensional binary optical flow is shown to have an angular dependence on the angle of the linear polarizer. It is further observed that by increasing the number of wire grid orientations, a digital representation of the polarization very similar to the analog can be obtained.

\subsection{Reference}

[6.1] E. Adelson and J. Bergen, "The plenoptic function and the elements of early vision," In M. Landy and J. Movshon (Eds.), Computational Models of Visual Processing, Cambridge, MA: MIT Press, pp. 3-20, 1991.

[6.2] J. Barron, D. Fleet, and S. Beauchemin, "Systems and experiment performance of optical flow techniques," International Journal of Computer Vision, vol. 12, no. 1, pp. 43-77, 1994.

[6.3] S. Negahdaripour and B. Horn, "Direct passive navigation," IEEE Transactions on Pattern Analysis and Machine Intelligence, vol. 9, no. 1, pp. 168-176, 1987.

[6.4] C. Anderson, P. Burt, and G. van der Wal, "Change detection and tracking using pyramid transformation techniques," Proceedings of SPIE-Intelligent Robotics and Computer Vision, vol. 579, pp. 72-78, 1985.

[6.5] I. Haritaoglu, L. Davis, and D. Harwood, "W4 who? when? where? what? a real time system for detecting and tracking people," International Conference on Automatic Face and Gesture Recognition, pp. 222-227, 1998.

[6.6] C. Wren, A. Azarbayejani, T. Darrell, and A. Pentland, "Pfinder: Realtime tracking of the human body," IEEE Transactions on Pattern Analysis and Machine Intelligence, vol. 19, no. 7, pp. 780-785, 1997.

[6.7] G. Doretto and S. Soatto, "Editable dynamic textures," IEEE Proceedings of Computer Society Conference on Computer Vision and Pattern Recognition, vol. 2, pp. 137-142, 2003.

[6.8] A. Elgammal, D. Harwood, and L. Davis, "Non-parametric model for background subtraction," IEEE Proceedings of European Conference on Computer Vision, pp. 751-767, 2000.

[6.9] A. Borst, "How do flies land? from behavior to neuronal circuits," BioScience, vol. 40, no. 4, pp. 292-299, 1990. 
[6.10] R. Brinkworth and D. O'Carroll, "Robust models for optic codeing in natural scences inspired by insect biology," PLoS Computational Biology, vol. 5, no. 11, pp. 1-14, 2009.

[6.11] J. Tanner and C. Mead, "An integrated analog optical motion sensor," VLSI Signal Processing II, New York: IEEE Press, pp. 59-76, 1986.

[6.12] T. Delbrück, "Silicon retina with correlation-based, velocity-tuned pixels," IEEE Transactions on Neural Networks, vol. 4, no. 3, pp. 529-541, 1993.

[6.13] J. Kramer, "Compact integrated motion sensor with three-pixel interaction," IEEE Transactions on Pattern Analysis and Machine Intelligence, vol. 18, no. 4, pp. 455-460, 1996.

[6.14] A. Moini, A. Bouzerdoum, K. Eshraghian, A. Yakovleff, X. Nguyen, A. Blanksby, R. Beare, D. Abbott, and R. Bogner, "An insect vision based motion detection chip," IEEE Journal of Solid-State Circuits, vol. 32, no. 2, pp. 279-283, 1997.

[6.15] R. T. Collins, A. J. Lipton, T. Kanade, H. Fujiyoshi, D. Duggins, Y. Tsin, D. Tolliver, N. Enomoto, O. Hasegawa, P. Burt, and L. Wixson, "A system for video surveillance and monitoring," CMU VSAM final report, pp. 1-69, 1999.

[6.16] P. Lichtsteiner, C. Posch, and T. Delbrück, "A 128128 120db 30mw asynchronous vision sensor that responds to relative intensity change," IEEE ISSCC Digest of Technical Papers, pp. 508-509, 2006.

[6.17] M. Innocent and G. Meynants, "Differential image senor with high common mode rejection," Proceedings of European Solid-State Circuits Conference, pp. 483-486, 2005.

[6.18] S. Shafie, S. Kawahito, I. Halin, and W. Hasan, "Non-linearity in wider dynamic range CMOS image sensors utilizing a partial charge transfer technique," Sensors, vol. 9, pp. 9452-9467, 2009.

[6.19] M. Srinivasan, S. Zhang, J. Chahl, E. Barth, and S. Venkatesh, "How honeybees make grazing landings on flat surfaces," Biological Cybernetics, vol. 83, pp. 171-183, 2000.

[6.20] A. Borst, "Correlation versus gradient type motion detectors: the pros and cons," Philosophical Transactions of the Royal Society, B: Biological Sciences, vol. 362, no. 1479, pp. 369-374, 2007. 
[6.21] W. Reichardt, "Autocorrelation, a principle for the evaluation of sensory information by the central nervous system," Principles of Sensory Communication, pp. 303-317, 1961.

[6.22] H. Okuno and T. Yagi, "Bio-inspired real-time robot vision for collision avoidance," Journal of Robotics and Mechatronics, vol. 20, no. 1, pp. 6874, 2008.

[6.23] R. Harrison, "A biologically inspired analog IC for visual collision detection," IEEE Transactions on Circuits and Systems-I, vol. 52, no. 11, pp. 2308-2318, 2005.

[6.24] M. B. Reiser and M. Dickinson, "A test bed for insect-inspired robotic control," Philosophical Transactions of the Royal Society, A: Mathematical, Physical and Engineering Sciences, vol. 361, pp. 2267-2285, 2003.

[6.25] T. Neumann and H. Blthoff, "Behavior-oriented vision for biomimetic flight control," Proceedings of the International Workshop on Biologically Inspired Robotics, pp. 196-203, 2002.

[6.26] H. Krapp, "Neuronal matched filters for optic flow processing in flying insects," Neurological Processing of Optic Flow, San Diego: Academic Press, pp. 93-120, 2000.

[6.27] S.Tao and L. Zeng, "An elementary-motion-detector based on hardware lateral inhibition network," Journal of Physics: Conference Series 48, pp. 212-216, 2006.

[6.28] D. Lee, "A theory of visual control of braking based on information about time-to-collision," Perception, vol. 5, no. 4, pp. 437-459, 1976.

[6.29] B. Horn and P. Schunck, "Determining optical flow," Artificial Intelligence, vol. 17, pp. 185-203, 1981.

[6.30] S. Decker, R. McGrath, K. Brehmer, and C. Sodini, "A 256 x 256 CMOS imaging array with wide dynamic range pixels and column- parallel digital output," IEEE Journal of Solid-State Circuits, vol. 33, pp. 2081-2091, 1998.

[6.31] N. Akahane, S. Sugawa, S. Adachi, K. Mori, T. Ishiuchi, and K. Mizobuchi, "A sensitivity and linearity improvement of a 100db dynamic range CMOS image sensor using a lateral overflow integration capacitor," Symposium on VLSI Circuits, Digest of technical papers, pp. 62-65, 2005.

[6.32] O. Yadid-Pecht and E. Fossum, "Wide intrascene dynamic range cmos aps using dual sampling," IEEE Transactions on Electron Devices, vol. 44, pp. 1721-1723, 1997. 
[6.33] D. Yang, A. E. Gamal, B. Fowler, and H. Tian, "A 640 x 512 cmos image sensor with ultra-wide dynamic range floating-point pixel level ADC," IEEE Journal of Solid-State Circuits, vol. 34, pp. 1821-1834, 1999.

[6.34] E. Buchner, "Elementary movement detectors in an insect visual system," Biological Cybernetics, vol. 24, pp. 85-101, 1976.

[6.35] J. van Hateren and H. P. Snippe, "Information theoretical evaluation of parametric models of gain control in blowfly photoreceptor cells," Vision Research, vol. 41, no. 14, pp. 1851-1865, 2001.

[6.36] E. Buchner, "Behavioural analysis of spatial vision in insects," Photoreception and Vision in Invertebrates, vol.74 of A: Life Sciences, New York, London, pp. 561-621, 1984.

[6.37] A. Borst and M. Egelhaaf, "Principles of visual motion detection," Trends in Neurosciences, vol. 12, no. 8, pp. 297-306, 1989.

[6.38] M. Sarkar, D. S. Segundo, C. van Hoof, and A. Theuwissen, "An analog and digital representation of polarization using CMOS image sensor," Proceedings of 5th European optical society tropical meeting on advanced imaging techniques, 2010. ISBN: 9783000305030. 


\section{7}

Conclusions and future work

In this thesis the design of a CMOS image sensor inspired by the compound eye of insects, as well as several applications of such image sensor are presented. The sensor is able to detect polarization information using a micro-polarizer oriented at various angles and created using the metal layers in a standard CMOS process. The detected polarization information is shown to be useful in classifying materials as well as detecting incoming light ray directions for use in autonomous agent navigations. Different algorithms used by the eyes of insects to detect motion were also studied and a model for collision detection has been proposed.

In this final thesis chapter some of the general conclusions of the work are summarized in section 7.1. Overviews of possible future works are listed in section 7.2. 


\subsection{Summary of achievements}

Biological systems can be an inspiration for the development of small autonomous sensor nodes. The eye of an insect is a compound eye with individual lenses and their own photoreceptor arrays. This visual system allows an insect to fly with limited intelligence and brain processing power. Among the compact vision systems in nature, compound eyes are the smallest known vision sensors. They exhibit the lowest possible volume and weight as well as low power consumption [7.1], [7.2]. For example the diameter of a single receptor in a bee is around $25 \mu \mathrm{m}$ thus the volume occupied is around $0.0005 \mathrm{~mm}^{2}$. For compound eyes with around 10000 of these receptors the area occupied is around $5 \mathrm{~mm}^{2}$ which is very small compared to the area of $2500 \mathrm{~mm}^{2}$ occupied by human retina. Their low spatial resolution is partly compensated by high temporal imaging rate and parallel signal processing. These eyes have a unique superiority in detecting fast moving objects and polarization information, making them ideal for vision sensors in machine vision applications.

\subsubsection{Sensing polarization information}

The eyes of insects are able to detect polarization information using the spatial arrangement of the visual pigments in the optical channel as seen in chapter 3. Similar to the pattern of the visual molecules a wire grid micro-polarizer was implemented using the standard metal layers of CMOS technology. One half of the pixel array of the designed image sensor was used to analyze the polarization information while the remaining half for normal vision applications. The polarization region was further split into two regions. The region 1 has a micro-polarizer oriented at $0^{\circ}$ and $90^{\circ}$, while region 2 also has micro-polarizer oriented at $45^{\circ}$.

The transmission efficiency and extinction ratio for the two polarization sense regions was measured for incident electromagnetic waves. The implemented micro-polarizer showed polarization sensitivity, and extinction ratios of 6.3 and 7.7 were achieved in the two polarization sense regions of the sensor. The extinction ratios of available external linear polarizers range from 10 to over $10^{7}$. Higher the extinction ratio, greater is the precision in detecting the polarization component of the incident light. As discussed in chapter 3 , the extinction ratio is a function of the wire grid pitch and with the scaling of the technology the pitch can be lowered and thus would help to achieve higher extinction ratios.

The metal grid on top of the photodiode can also affect the wavelength 
responsivity. The one-dimensional metal pattern shows high transmittance but little or no wavelength selectivity in the visible wavelength range, while twodimensional metal patterns show high selectivity but low transmittance. Using the pattern of metal layers it is not only possible to detect polarization but also obtain a wavelength selective (color) filter inside the pixel, which would enable the elimination of additional color filter processing steps or thin films. The use of metal grid would severely attenuate the light and thus these image sensors would not be good for high sensitivity imaging. However they would be of interest in some machine vision applications where the polarization and color are derived separately using multiple filter layers, which severely affects the light efficiencies. The metal layers would provide both the polarization and color information and thus would aid to increase the light efficiency in those applications.

\subsubsection{Using polarization information in material classifica- tion}

As seen in chapter 4 , it is possible to classify among reflecting surfaces based on the polarization of the reflected light. The polarization state for the diffuse and specular components of the reflection depends on the reflecting surface. The magnitude of variations in the maximum and minimum transmitted irradiances due to the variation in the reflection pattern of metal and dielectric surfaces was found to be useful in classifying among them. The variation in the transmitted irradiance for aluminum and plastic surfaces was shown, which can be used to model an image sensor for real time material classification.

Various other measurement metrics, such as the degree of polarization (DOP) and the polarization Fresnel ratio $(P F R)$, were shown to measure the variations in the reflection pattern of the metal and dielectric surface. The degree of linear polarization obtained using Stokes parameters is also shown to be able to classify surfaces into metal and dielectrics.

The polarization of the reflected component of the light wave varies with the conductivity of the metallic surface and this was further explored and shown to be able to serve as a tool to classify metallic surfaces as highly conductive and lowly conductive.

\subsubsection{Using polarization information in navigation}

In the egocentric form of navigation often used by insects, the direction of travel is determined from the position of the sun or from skylight polarization. 
Polarization in the sky occurs because the sunlight is scattered by atmospheric molecules. The direction of polarization ( $e$-vector) and the degree of polarization observed in the sky rotates about the zenith with the position of the sun. The intensities measured using the embedded micro-polarizers in the pixel can be used to calculate the degree of polarization and polarization Fresnel ratio. As the degree of polarization varies with the change in position of the sun, this variation can be used as a compass (chapter 5). The transmitted intensities of the wire grid micropolarizer can also be used to calculate the Stoke parameters, which can be further used to obtain the positional information of the light source.

The ellipticity and azimuthal angles computed using the Stokes parameters of the perceived light ray are shown to be correlated to the incoming light ray direction. A correlation coefficient of 0.94 or higher was obtained in all the measurements.

A navigational sensor based on the polarization of light would be independent of the visual cues and use natural light to determine the directional reference.

\subsubsection{Regarding motion/collision detection}

The most popular bio-inspired visual guided motion detector is the correlationtype elementary motion detection $(E M D)$ proposed by Hassenstein and Reichardt [7.3]. It computes the optic flow by correlating the response of one photoreceptor to the delayed response of an adjacent photoreceptor, both looking in the same direction. The process of obtaining the optic flow is quite complex and needs complicated circuits.

In order to have compact and low-power biologically inspired motion detection systems, the estimation of the optic flow has to be simplified. The designed image sensor when operating either in temporal differential mode or spatial integration of one-dimensional binary optic flow mode was shown to be able to detect collision of moving objects with itself (chapter 6). The binary optic flow is generated in-pixel from multiple images stored in the in-pixel memories and spatially integrated using a counter.

The computations of the binary flow are relatively easy and are shown to be able to detect collision successfully. This allows for the design of simple, miniaturized, low power and narrow path navigation of autonomous agents. 


\subsection{Future works}

The field of view of an imaging polarimeter is usually limited by the imaging optics. Commercially available photographic and video cameras have a field of view of about $30^{\circ}-50^{\circ}$ (horizontal) by $20^{\circ}-40^{\circ}$ (vertical) depending on the focal length and aperture. Ideally $180^{\circ}$ field of view imaging polarimetry is desired to study the polarization patterns.

A $124^{\circ}$ field of view camera with less than $1^{\circ}$ angular resolution has been discussed in chapter 2. Such a camera would help to increase the sensitivity of the polarization measurements while allowing to gather wide field of view information. A multichannel imaging system is discussed along with its possible applications in the following subsections.

\subsubsection{Multichannel imaging system}

Leonardo da Vinci [7.4] stated that "every body in the light and shade fills the surrounding air with infinite images of itself; and these, by infinite pyramids defused in the air, represent this body throughout space and on every side" as shown in figure 7.1.

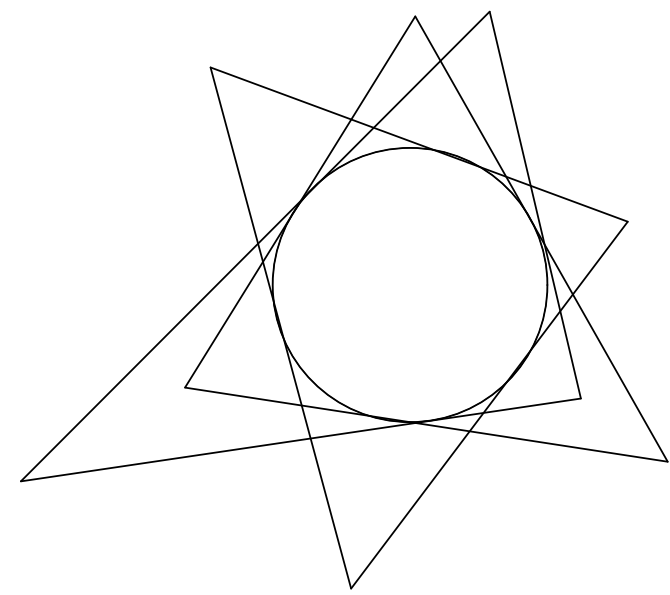

Figure 7.1: Diagram of optical "pyramids" from Leonardo's notebook [7.5].

In chapter 6, the plenoptic function used for the visual representation of the scene was described. This function changes differentially over time. Each of the "pyramids" in figure 7.1 denotes a subset of the plenoptic function at a given time which can be captured by an imaging device. 
In the conventional imaging approach using a single pinhole camera, only one of the pyramids in its field of view is visible and thus forms a single image. A single-axis lens focuses an image on the focal plane of the image sensor, the resolution of which is determined by the size of the optical window. Through this arrangement, cross talk, i.e. the coupling of one signal into the adjacent channel is negligible even for significant aberrations or misalignment errors and a high resolution is obtained over a continuous area. However conventional imaging systems are not able to convey the shape and size of the object of interest. In the multichannel aperture imaging system discussed in section 2.2, captures the plenoptic function from many different viewing points.

Multichannel electro-optical systems are superior in detecting motion and determining the shape of the object in motion. To determine the structure of an object from motion two conditions need to be satisfied: high field of view and linearity of estimation [7.6]. In single pin-hole cameras, one ray from each point in space is captured and from this ray at different times the structure of motion must be estimated. This makes the estimation of the viewing geometry a non-linear problem. The additional information in the multichannel imaging system (multiple rays from the same scene point) makes estimation of the viewing geometry linear.

In chapter 2, the micro-optical lens designed by the Vrije Universiteit Brussel $(V U B)$ which is to be integrated with the designed image sensor was described. To sample a wide field of view ( $F O V$ ) of $124^{\circ}$, an overlap of the different angular regions mapped by the separate optical channels is allowed which results in distorted images. However it is possible to reconstruct the single image from the different distorted image regions as there is a direct relationship between the angle of incidence and the position of the detector. In the following sub-section the imaging mapping and distortion correction methods are discussed.

\subsubsection{Image mapping and distortion correction}

The captured images from the multiple lens optical system are processed to reconstruct the proper image. Each optical channel captures light from a certain $F O V$, but due to the mapping of spherical angular distribution onto a square detector, overlapping of the FOVs is not completely avoidable. Thus certain directions will be sampled by more than one pixel of the CMOS detector. This introduces non-linearities or distortion in every zone by the optical system, as shown in figure 7.2. The figure shows the simulated spot resolution on the detector for channels one to nine from the designed 25 channels as described 
in section 2.3. The spot distribution obtained by the different optical channels are well separated which shows that there are no stray-light. Furthermore the energy decreases towards the outer region of the sub-FOVs due to vignetting. In ideal case the spots in each optical channel should be easily distinguishable, however due to the distortion of the optical channel nonlinearities are introduced deforming the shape of the optical channels.
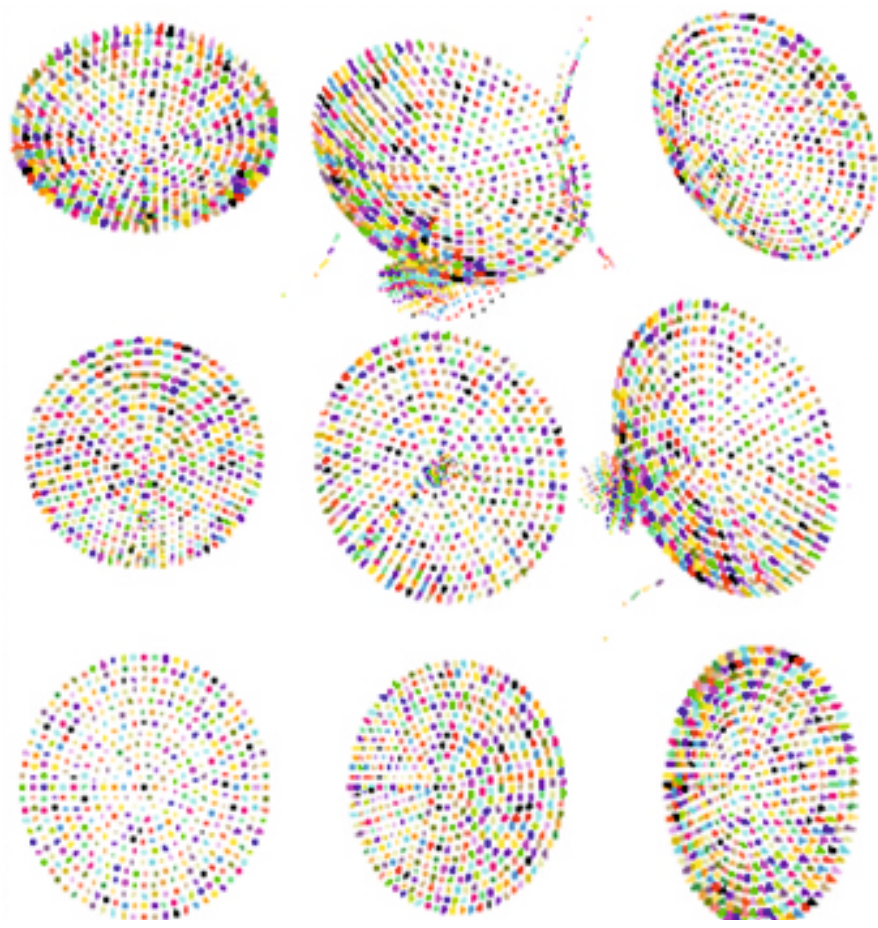

Figure 7.2: Obtained distorted sub-image from the nine optical channels for the designed lens array.

Nonlinearities of an optical system can be largely described by two types of distortion: lens geometric distortion and photometric distortion. The photometric distortion refers to a position-dependent loss of light in the output of the optical system. It is caused by the varying $P S F$ at each pixel due to blurring, scattering of light rays or overlapping of the light rays. This degrades the spatial resolution of the system's final image and it causes gradual fading of the image points near the periphery. The geometric distortion is caused by the variation in the focusing distance. The projection function of a multi lens camera can be observed as that of a projection from a perspective (central) camera, followed by a non parametric 
displacement of the imaged point in the direction of the distortion center [7.7]. The displacement of the imaged points induces a non-linear distortion, i.e. straight lines in space are not imaged as straight line unless passing through the center.

Image mapping is limited by the nonlinearities of the optical system. Image mapping is defined as the direct correspondence of the image at the pixel and the viewing direction. A high resolution image can be obtained from the set of low resolution images by pixel rearranging. However it is important to remove the photometric and geometric nonlinearities before trying to rearrange the subimages pixels on the virtual image.

The photometric distortion can be corrected using the model proposed by [7.8]. The output image $g(x, y)$ can be expressed as

$$
g(x, y)=f(x, y) * h(x, y)+n(x, y)
$$

where $f(x, y)$ is the original object on the image plane, $n(x, y)$ is noise, $h(x, y)$ is the system's point spread function (PSF) containing the information of the degradation introduced by the system. The noise and the PSF for each lens channel is known, thus the original image $f(x, y)$ can be easily obtained.

Geometric distortion can be further sub-classified into radial distortion, decentering distortion and thin prism distortion. Radial distortion is caused by imperfect lens shape and manifests itself as radial positional error. Decentering distortion and thin prism distortion are caused by the improper lens and camera assembly, generating both radial and tangential errors in point position. Among the three forms of geometric distortion, radial distortion is of prime concern. In figure 7.2, two types of radial distortion are observed: pincushion and barrel. Pincushion distortion distorts a square object into a pincushion shape while barrel distorts a square object into more of a barrel shaped object. The barrel distortion is more dominant in a lens system, mainly in lenses with short focal length.

Radial distortion is strictly symmetrical about the optical axis of the lens and is governed by the equation [7.9]

$$
r^{\prime}=r+k_{1} r^{3}+k_{2} r^{5}+k_{3} r^{7} \ldots
$$

Equation (7.2) can be safely approximated by using only the first term of the infinite series as

$$
r^{\prime}=r+k_{1} r^{3}
$$


where $r^{\prime}$ is the distorted radius, $k$ is the coefficient of radial distortion and $r$ is the original radius. The sign of $k$ in equation (7.3) affects the type of radial distortion. If $k$ is negative then it is a barrel radial distortion and if positive then it is a pincushion radial distortion.

In order to correct for the radial distortion, the center of the radial distortion and the coefficient of radial distortion are to be computed. The center of the distortion is not the center of the image. However, as the true position is not well constrained and it is necessary to fix it somewhere, the center of the image turns out to be a good approximation [7.10]. The center of the sub-image needs to be calibrated with high accuracy for two reasons:

a) Each microlens has a relatively small field of view with a dimension of around $25 \times 25$ pixels in the designed electro-optical system. Thus if the image center calculation varies by approximately five pixels, an error of $20 \%$ is introduced in the image reconstruction, which is quite high. A one pixel variation of the center point would reduce the error to $4 \%$.

b) Accurate image center position leads to the prediction of accurate position of the optical axis for each eyelet. The radial distortion is symmetrical around the optical axis, thus the accurate determination of the optical axis would help to eliminate or reduce the radial distortion.

The sub-image center point can be determined using various methods. Most algorithms in literature use the center of the image buffer frame as the center of the sub-image. This method introduces considerable error compared to the field of view. The other common method used in the literature is the center point detection using vanishing point. By prospective projection, all parallel lines in the object space except the lines parallel to the image plane will appear to intersect at a point called a vanishing point $P$ as described in [7.11]. However this involves large scale computations and the goal is to have an on-chip mapping based on the scene of the image in the field of view of the eyelet with less computational overload.

The best method for an accurate on-chip sub-image center point computations is image centroiding. The image center is computed using the following equations [7.12]:

$$
\begin{aligned}
C_{x} & =\sum \frac{I_{i} \times x_{i}}{I_{i}} \\
C_{y} & =\sum \frac{I_{i} \times y_{i}}{I_{i}}
\end{aligned}
$$


where $C_{x}, C_{y}$ are the centroid points, $I_{i}$ can be taken as the photo detector voltage at pixel $i$ and $x_{i}$ and $y_{i}$ are horizontal and vertical position of the pixel $i$.

The possibility of on-chip image correction and reconstruction from the multiple sub-images would drastically reduce the bandwidth of the sensor, contributing to low power mode of operation. Such a system would enable us to reduce the volume of the imaging optics and pave the way to realize a compact image capturing system.

\subsubsection{Wide field of view imaging system with polarization sensitivity}

Wide field of view imaging systems can potentially improve applications such as security surveillance, robot navigation or endoscopy. Such a system can collect image data points from a relatively larger area than the rectilinear lenses and thus can be employed in both contact and contactless endoscopy. The fish eye lenses which are generally used in endoscopy for images produce a significant distortion in the visual content. Contactless endoscopy is becoming the standard in the endoscopy world. The image sensor with wide field of view, high angular sensitivity and polarization sensitivity would enhance the application areas of the endoscopes.

The common requirements for a wireless capsule endoscope are high resolution, very low power, high dynamic range and high sensitivity. Resolution is needed to be able to see things clearly inside the digestive tract or the intestines. The state-of-the-art commercial wireless capsule endoscope product, the PillCam capsule, developed by Given Imaging Ltd transmits 256x256x8 bit data at a frame rate of 2 frames per second [7.13]. The sensor described in chapter 3 is able to transmit $128 \times 128 \times 12$ bits of data at a frame rate of $15 \mathrm{fps}$. Besides resolution, endoscopy applications demand a dynamic range of $60-80 \mathrm{~dB}$ which is easily achievable. The dynamic range can be varied using the partial charge transfer as seen in chapter 6 . The ability to do in-pixel processing of the data helps in data compression, which naturally helps to lower the output data rate and lower the power consumption of the system.

The ability of the designed sensor to detect polarization of the reflected light adds value to medical imaging. It is known that when light interacts with tissue, the reflectance, absorption and polarization of light is affected. The polarization parameters of light scattered from biological tissues contains rich morphological and functional information useful for medical purposes. Potential applications of such electro-optical system include imaging of superficial cancers 
and other skin lesions, early detection of diseased cells and microscopic analysis of tissues [7.14].

A potential application of linear polarization imaging techniques in dermatology has been proposed by [7.15]. This technique records a series of images corresponding to different combinations of illumination and polarization and calculates the intensity difference between orthogonal direction polarizations pixel by pixel. The degree of polarization information obtained from the surrounding muscles or blood can also be evaluated. In chapter 3, it was shown that it is possible to detect polarization information using metallic wire grids and it is also possible to compute the orthogonal polarization intensity difference and degree of polarization of polarization pixel by pixel. For the current version, these calculations are done off-chip but they can be easily incorporated onto the chip, which will further help in miniaturization of the endoscopes.

Besides endoscopy, wide field of view optical systems can also be used in imaging fluorescence microscopy. Fluorescence microcopy is used to investigate dynamic phenomenon in cells and living tissues and have seen dramatic increase in life science application in the last decade. Conventional CCD/CMOS cameras are not able to image the time-critical cellular events which change dynamically in less than a few seconds. To be able to capture these fast changes, multiple channel imaging optics are required to simultaneously track changes in the fluorescence signals. Besides fluorescence microscopy, fluorescence polarization imaging is also becoming popular in investigating the biochemical properties of samples, such as protein denaturation, protein-ligand interactions, protein-protein interactions, protein-DNA interactions, and the rotational rates of proteins [7.16], [7.17]. A wide field of view optical system with polarization sensitivity is ideal for biological investigations because it provides the ability to image simultaneously both the parallel and perpendicular polarization components of a fluorescence emission.

\subsubsection{High angular resolution imaging system with polar- ization sensitivity}

In chapter 3, it was elaborated that the performance parameters of a wire grid micro-polarizer depend on the pitch used. To obtain a high transmission efficiency and extinction ratio $(E R)$ in the visible region, the pitch has to be smaller than the incident wavelength. The $0.18 \mu \mathrm{m}$ CMOS technology used to fabricate the designed image sensor provides a minimum metal spacing of $0.24 \mu \mathrm{m}$. Nevertheless, as CMOS technologies keep scaling and smaller feature 
sizes become available, it is possible to fabricate the suggested structures with smaller spacings.

The polarization sensitivity also depends on the wavelength of the incident electromagnetic wave and the angle of incidence. As wavelength and grating spacing becomes nearly equal, the angle of incidence of the light ray has more pronounced effects on the polarization detection sensitivity. In the case of a wide field of view lens with $1^{\circ}$ angular resolution, it offers two advantages. Firstly, the polarization ratio for a specific angle of incidence is known and secondly, it is possible to spatially sum the polarization response over a wide field of view. For the current version of the sensor, the polarization profile was measured for only a single angle of incidence; however after the integration of the lens the polarization measurement for the entire field of view can be obtained which will increase the resolution and sensitivity of the polarization measurement.

\subsubsection{Real time material classification}

In chapter 4 , it was shown that it is possible to classify materials based on the polarization information from the reflected surface. To keep the system simple, the degree of polarization and $P F R$ calculations were done off-chip. These calculations can be easily implemented on-chip, allowing for real time material classification. Further the transmitted intensities of the reflected light were measured for a single angle of incidence of the light ray. The measurement of the polarization Fresnel ratio $(P F R)$ at each pixel is not very accurate when the diffuse component of the reflection dominates over the specular component. In such an scenario the measurement of the reflected transmitted intensities for varying angle of incidence of the light ray would serve to increase the resolution and the sensitivity of the PFR measurement.

The multichannel imaging system with an angular resolution of $1^{\circ}$ would provide the polarization information of the reflected light over a wide field of view, thus serving as a more reliable measurement and classification system. The increased angular resolution with corresponding increase in the number of Fresnel reflection coefficients can provide a more intuitive way of investigating the specular and diffuse reflection changes as a function of physical parameters of the reflecting surface.

Furthermore, in this work only the differential changes in the intensities of the parallel and perpendicular component of the specular and diffuse reflections were explored and not the phase difference. Metals alter the phase of polarization of the incident light upon specular reflection while dielectrics do not [7.18]. This 
is important in the scenario of highly diffuse dielectric materials, which would often be misclassified as metals when using PFR. Ways to determine the change in phase of the incident and the reflected light by the reflecting surface need to be explored.

\subsubsection{Real time navigation}

In chapter 5, it was stated that insects use the egocentric form of navigation which computes the home vector from the direction of travel and the distance travelled. The possibility of using polarized light as a compass to determine the direction of travel was shown. The distance of travel computation were not discussed, however they are not very difficult to compute. One such method is proposed here which can be easily implemented on chip for distance of travel calculations.

Assuming the initial position of the object to be $(x, y, t)$ then if the point has travelled to a new location after a time $\Delta t$ the new location $X$ and $Y$ can be described as

$$
\begin{aligned}
& X(t+\Delta t)=x(t)+\cos \theta(t) \times v(t) \times \Delta t \\
& Y(t+\Delta t)=y(t)+\sin \theta(t) \times v(t) \times \Delta t
\end{aligned}
$$

where $\theta$ is the angle of travel and $v(t)$ is the velocity of travel. $\Delta t$ is the integration time to obtain the next frame information. The velocity can be computed from the optic flow as discussed in chapter 6 . However the computation of the velocity from the optic flow would be computationally demanding. The velocity of travel is proportional to the change in the intensity of the image over time [7.19] and is expressed as

$$
\Delta i \approx \Delta v \times \Delta t
$$

where $\Delta i$ is the change in intensity over the time interval $\Delta t$. Substituting equation (7.8) in equations (7.6) and (7.7) we get

$$
\begin{aligned}
& X(t+\Delta t)=x(t)+\cos \theta(t) \times \Delta i \\
& Y(t+\Delta t)=y(t)+\sin \theta(t) \times \Delta i
\end{aligned}
$$


The new position is depended on the angular travel and the change in intensity during the time $\Delta t$. The change in intensity can be computed from the difference in two frames separated by $\Delta t$ using partial charge transfer as discussed in chapter 6 , while the angular information can be obtained from the compass discussed in chapter 5 . Thus it would indeed be possible to have both the direction and distance information which would simplify the algorithms currently used for autonomous agent navigation.

\subsubsection{Real time sun position detection}

The proposed design will make it possible for an alternate method of angular position determination of the sun besides the slit sun digital sensors. The celestial compass based on skylight polarization is described in chapter 5. Depending on the angle the light from the sun hits the atmosphere; the light is polarized in a special way. These patterns maintain an interesting characteristic all over the day: they are always perpendicular to the solar meridian. Thus the degree of polarization has a maximum when the sun is at $90^{\circ}$ angular position (with respect to solar meridian it is at $0^{\circ}$ ). It was shown that it is indeed possible to determine the elliptical and azimuthal position of an object based on the polarization patterns of the incoming light ray.

Due to the measurement setup constraints, it was not possible for the first version of the designed image sensor to test for the variability of the degree of polarization with respect to the angular position of the sun under open sky. The measurement setup can be simplified and an open sky measurement should be a valuable extension of the project. The study of the change of the degree of polarization with the position of the sun would immensely help in the navigation of autonomous agents which usually depend on heavy computations to determine the angle of travel. Further it is known that sun moves at a constant angular velocity. The change from the solar meridian (the point of highest degree of polarization) and the calculated orientation would also be able to predict the time elapsed.

A comparison between different sensors to determine the sun position is shown in the table 7.1 [7.12]. 
7.3. Reference

\begin{tabular}{|c|c|c|c|c|}
\hline & GPS & Magnetometer & SSC [7.12] & proposed \\
\hline Mass & $\sim 20 \mathrm{gm}$ & $\sim 50 \mathrm{gm}$ & $\begin{array}{l}\sim 20 \mathrm{gm} \\
\text { including } \\
\text { processor }\end{array}$ & same as SSC \\
\hline Power & $\sim 0.5 \mathrm{~W}$ & $\sim 0.3-0.5 \mathrm{~W}$ & $\sim 0.2 \mathrm{~W}$ & $\sim 0.1 \mathrm{~W}$ \\
\hline $\begin{array}{l}\text { Acquisition } \\
\text { time }\end{array}$ & $1 \mathrm{~min}$ & $1 \mathrm{sec}$ & $10 \mathrm{~min}$ & $10 \mathrm{~ms}-1 \mathrm{sec}$ \\
\hline Accuracy & $0.15^{\circ}-0.5^{\circ}$ & $\begin{array}{l}0.5^{\circ} \text { if } \\
\text { position is } \\
\text { known }\end{array}$ & $0.1^{\circ}$ & $\begin{array}{c}\text { Minimum } \\
\text { of } 1^{\circ}\end{array}$ \\
\hline $\begin{array}{l}\text { Operation on } \\
\text { moving } \\
\text { vehicles }\end{array}$ & Yes & Yes & No & Yes \\
\hline $\begin{array}{c}\text { Operational } \\
\text { constraint }\end{array}$ & $\begin{array}{c}\text { GPS } \\
\text { coverage }\end{array}$ & $\begin{array}{c}\text { Initial position } \\
\text { must be known. } \\
\text { Will not } \\
\text { operate in } \\
\text { polar regions }\end{array}$ & $\begin{array}{l}\text { Needs to } \\
\text { see the sun }\end{array}$ & $\begin{array}{l}\text { High extinction } \\
\text { ratio for } \\
\text { increased } \\
\text { polarization } \\
\text { sensitivity }\end{array}$ \\
\hline
\end{tabular}

Table 7.1: Comparison of sun position detection sensors.

\subsection{Reference}

[7.1] P. Shoemaker and D. O'Carroll, "Insect small-target motion detection for seeker applications," tech. rep., Final report, Tanner Research, Inc., 2003.

[7.2] J. Zufferey, Bioinspired vision based flying robots. PhD thesis, EPFL, 2005.

[7.3] W. Reichardt, "Autocorrelation, a principle for the evaluation of sensory information by the central nervous system," Principles of Sensory Communication, Wiley, New York, pp. 303-317, 1961.

[7.4] J. Richter, The Notebooks of Leonardo da Vinci, vol. 1, p. 39. New York: Dover, 1970.

[7.5] E. Adelson and J. Wang, "Single lens stereo with a plenoptic camera," IEEE Transaction on Pattern Analysis and Machine Intelligence, vol. 14, no. 2, pp. 99-106, 1992.

[7.6] J. Neumann, C. Fermueller, and Y. Aloimonos, "Eyes from eyes: analysis of camera design using plenoptic video geometry," tech. rep., Technical Report CAR-973, 2001. 
[7.7] J. P. Tardiff, P. Strum, and S. Roy, "Plane based self calibration of radial distortion," Proceedings of International Conference on Computer Vision, pp. 1-8, 2007.

[7.8] R. Tudela, A. Brueckner, J. Duparre, and A. Braeuer, "An image restoration approach for artificial compound eyes," Proceedings of Electronic Imaging SPIE, vol. 6812, pp. 681200.1-681200.1, 2008.

[7.9] R. Tsai, "An efficient and accurate camera calibration technique for $3 \mathrm{~d}$ machine vision," Proceedings of Conference on Computer Vision and Pattern Recognition, pp. 364-374, 1986.

[7.10] B. Tordoff and D. Murray, "Violating rotating camera geometry: The effect of radial distortion on self-calibration," Proceedings of International Conference on Pattern Recognition, vol. 1, pp. 423-427, 2000.

[7.11] D. Forsyth and J. Ponce, Computer Vision a modern Approach. New Jersey: Prentice Hall Inc, 2nd. ed. ed., 2003. ISBN: 9780130851987.

[7.12] C. Liebe, "Solar compass chip," IEEE Sensors Journal, vol. 4, no. 6, pp. 779-786, 2004.

[7.13] Y. Metzger, S. Adler, A. Shitrit, B. Koslowsky, and I. Bjarnason, "Comparison of a new pillcam sb2 video capsule versus the standard pillcam sb for detection of small bowel diseases," Reports in Medical Imaging, vol. 2, pp. 7-11, 2009.

[7.14] N. Ghosh, J. Soni, M. Wood, M. Wallenberg, and I. Vitkin, "Mueller matrix polarimetry for the characterization of complex random medium like biological tissues," Pramana - Journal of Physics, vol. 75, no. 6, pp. 10711086, 2010.

[7.15] Z. Nan, J. Xiaoyu, G. Qiang, H. Yonghong, and M. Hui, "Linear polarization difference imaging and its potential applications," Applied Optics, vol. 48, no. 35, pp. 6734-6739, 2009.

[7.16] K. Elgass, K. Caesar, F. Schleifenbaum, Y. Stierhof, A. Meixer, and K. Harter, "Novel application of fluorescence lifetime and fluorescence microscopy enables quantitative access to subcellular dynamics in plant cells," PLOS ONE, vol. 4, no. 5, pp. e5716-1-13, 2009.

[7.17] G. Mashanov, T. Nenasheva, M. Peckham, and J. Molloy, "Cell biochemistry studied by single molecule imaging," Biochemical Society Transactions, vol. 34, no. 5, pp. 983-988, 2006. 
[7.18] H. Chen and L. Wolff, "Polarization phase-based method for material classification in computer vision," International Journal of Computer Vision, vol. 28, no. 1, pp. 73-83, 1998.

[7.19] L. Lopez, "Neural processing for control of artificial compound eye," IEEE International Conference on Computational Intelligence, vol. 5, pp. 2749-2753, 1994. 


\section{Summary}

Biological systems are a source of inspiration in the development of small autonomous sensor nodes. The two major types of optical vision systems found in nature are the single aperture human eye and the compound eye of insects. The latter are among the most compact and smallest vision sensors. The visual system of insects allows them to fly with a limited intelligence and brain processing power. A CMOS image sensor replicating the perception of vision by insects is discussed and designed in this thesis for industrial (machine vision) and medical applications.

Visual perception is the ability to detect light and interpret it. In chapter 1 of the thesis different vision perceptions found in the animal kingdom are briefly described. Among the known optical vision systems in nature, the single aperture eye is more popular with camera researchers for its ability to form high resolution images. Compound eyes are typically not suitable for high resolution images. However they are known to process information more efficiently and are good examples of low power vision systems. Light emanating from reflections is partially polarized. Single aperture eyes are polarization blind, but compound eyes are able to detect polarization. The compound eyes are also more suited for motion detection, have high sensitivity in low light conditions and offer wide field of view at a cost of low optical resolution. Though the compound eyes are not the best in terms of image quality, the advantages that they offer makes them better suited for machine vision applications than the single aperture eyes.

Compound eyes are composed of many ommatidia or facets. The natural compound eyes are classified broadly into apposition and superposition eyes. In apposition eyes each facet receives light only from one direction while in superposition eyes the light rays from different facets are superimposed. To design an artificial compound eye inspired by the natural compound eye, a microoptical lens array and a detector array are required. In chapter 2 , a realization of a 
single camera system wherein the scene is optically distributed across multiples lenses using a single detector array is presented. To optically distribute the scene, a compound lens structure has been designed by Vrije Universiteit Brussel (VUB) within the project of which this work is also part of. The designed lens has a field of view of $124^{\circ}$, a maximum contrast of 0.3 line pairs per degree $\left(\mathrm{LP} /{ }^{\circ}\right)$ and an angular resolution of less than $1^{\circ}$.

In chapter 2, the design of the detector array for said optical system is presented. The designed image sensor consists of an array of 128 by 128 pixels, it occupies an area of $5 \mathrm{~mm} \times 4 \mathrm{~mm}$ and it has been designed and fabricated in the $0.18 \mu \mathrm{m}$ CMOS CIS process from UMC. The pixel used in the design is a smart pixel, which is a form of an active pixel sensor. Each pixel contains a pinned photodiode $\left(p^{+} / n / p\right)$ and 33 transistors to perform low level image processing. The size of the pixel is $25 \mu \mathrm{m} \times 25 \mu \mathrm{m}$ and the size of the photodiode is $10 \mu \mathrm{m} \times 10 \mu \mathrm{m}$ which corresponds to $16 \%$ fill factor. The pixel also has an in-pixel analog and digital memory, which enables in-pixel digitization and realtime dynamic range adaptation. The designed image sensor can be operated in two modes: double differential mode and differential imaging mode. In case of double differential mode each pixel samples the voltages at the photodiode node twice: once after reset and again after the exposure period. In the differential mode two samples are obtained after different exposure periods.

In the designed image sensor only half of the available pixel array of 128 by 128 is used for normal imaging. The other half of the pixel array is used to analyze polarization information using metallic wire grid structures discussed in chapter 3. The three basic characteristics of light are intensity, color and polarization. Polarization vision and the mechanisms of the polarization of light from unpolarized light beam along with the representation of the polarization information are also discussed in detail in chapter 3. Polarization vision is a generalization of intensity and is analogous to color vision. In section 3.5 a metallic wire grid micropolarizer operating in spatial mode is presented which is designed using the metal layers available with the standard CMOS technology. Normally such a wire grid structure would function as a simple diffraction grating but when the pitch of the wires is less than half the wavelength of the incoming light, it becomes a polarizer. The designed polarization sensing sensor has an embedded linear polarizer in each pixel with varying orientations. The linear wire grid polarizer is implemented using thin metal strips with a line/space of $240 \mathrm{~nm} / 240 \mathrm{~nm}$ (pitch of $480 \mathrm{~nm}$ ). The absorption of the EM waves to completely $s$-polarize or $p$-polarize the transmitted wave through the wire grid is dependent on the pitch of the wire grid and so the performance of the wire grid as a polarizer 
also varies with the pitch. With the scaling of the CMOS technologies, the pitch of the wire grid will also scale thus improving their performance as a polarizer.

The pixel array used for polarization sensing is further split into two 64 by 64 pixel regions designated sense regions 1 and 2 . In sense region 1 , the wire grid is oriented horizontally and vertically to measure the $0^{\circ}$ and $90^{\circ}$ polarization intensity. While in sense region 2 beside the horizontal and vertical orientations the wire grid oriented in $45^{\circ}$ is also present. Linear polarizers are characterized by two main specifications: transmittance and extinction ratio. In the sense region 1 , for $0^{\circ}$ polarization the maximum and the minimum transmittances observed are $38.9 \%$ and $7 \%$ respectively. For $90^{\circ}$ polarization the maximum and the minimum transmittances observed are $44.4 \%$ and $0.1 \%$ respectively. Similarly, in sense region 2 for $0^{\circ}$ polarization the maximum and the minimum transmittances observed are $38.4 \%$ and $5.4 \%$ respectively while for $90^{\circ}$ polarization the maximum and the minimum transmittances observed are $42.4 \%$ and $0.6 \%$ respectively. The extinction ratios obtained in the sense region 1 and 2 are 6.3 and 7.7 respectively. These extinction ratios are higher than reported in literature for configuration similar to the designed sensor.

In chapter 4, material classification using polarization information is presented. Materials are broadly classified into metals and dielectrics. The classification of the material type can provide vital information about the scene in computer or machine vision. Unpolarized light becomes partially polarized after specular reflection. The state of polarization for the diffuse and specular components of the reflection depends on the reflecting surface and the measurement of the state of polarization of the reflected light serves as an indicator for the type of material surface. The magnitude of oscillations of the maximum and minimum transmitted irradiance due to the variations in the reflection pattern of the metal and dielectric surface was shown to be useful in classifying them. Various other measurement metrics, such as the degree of polarization and the polarization Fresnel ratio were also shown to measure the variations in the reflection pattern of the metal and dielectric surface. The Stokes degree of polarization was also evaluated to classify materials. The polarization of the reflected light also varies with the conductivity of the metallic surface and this was further explored and was shown to be able to serve as a tool to classify among highly conductive and lowly conductive metallic surfaces.

In chapter 5, navigation using polarized light is discussed. The navigational strategies of insects using skylight polarization are interesting for applications in autonomous agent navigation because they rely on very little information. The polarization pattern in the skylight varies in a systematic fashion both in 
$e$-vector and degree of polarization, according to the position of the sun. The Stokes parameters are used to calculate the ellipticity and azimuthal angles of a Poincaré sphere and these angles are shown to be correlated to the incoming light ray direction. A correlation coefficient higher than 0.94 was obtained in all the measurements. This method thus provides a way to determine the incoming polarized light direction which could be used to determine the angular positional information useful for autonomous agent navigation. The ability to compute onchip or in real-time the positional information would result in highly miniaturized navigational sensors and saving computational power. Further the degree of polarization was shown to vary with the polarization of the incoming light ray direction which could also serve as a compass for autonomous agent navigation algorithms. The variation in the degree of polarization with the position of the sun can further be explored in sun position detection based on skylight polarization.

In chapter 6 the ability of the insect to detect fast motion is explored and also the concept of representing polarization information in binary format is presented. The ability of flying insects to detect fast motion in the visual scene and avoid collision using low level image processing and little computational power makes their visual processing interesting for real time motion/collision detection in machine vision applications. The designed image sensor can also be operated in temporal differential mode and spatial integration of one-dimensional binary optical flow to detect motion/collision of moving objects replicating the "flickering effects" of the insects. The binary optical flow is generated in-pixel from multiple images stored in the in-pixel memories and spatially integrated using a counter. Both horizontal and vertical motions are easily detected using the binary optical flow which is computed relatively easily in the pixel. A collision alert algorithm to an approaching object as close as $2 \mathrm{~cm}$ is demonstrated. Such a collision detection algorithm would allow for the design of simple, miniaturized, low power and narrow path autonomous navigating agents. The designed sensor when operated in differential imaging mode provides for a background illumination invariant motion detection system. The generated binary optical flow is shown to have an angular dependence to the linear polarizer and with increasing the wire grid orientations the digital representation of the polarization resembles the analog form.

In chapter 7, along with the summary of achievements a multichannel imaging system was discussed. The integration of the compound lens from $V U B$ with the designed detector array would result in a multichannel imaging system. With high field of view and a low angular resolution, these optical vision systems would find applications not only in machine vision but also in medical applications. 


\section{Samenvatting}

Biologische systemen zijn een bron van inspiratie in de ontwikkeling van kleine autonome sensor nodes. De belangrijkste types van optische vision-systemen zijn in de natuur aanwezig: het enkelvoudige menselijk oog en het facet-oog van insecten. De laatstgenoemde behoort tot de meest compacte en kleinste visionsensoren. Het visuele systeem van insecten stelt hen in staat om te vliegen met een beperkte intelligentie en hersencapaciteit. In dit proefschrift wordt een CMOS-beeldsensor die de perceptie van het gezichtsvermogen bij insecten nabootst, besproken en ontworpen. De beeldsensor is geschikt voor industriële (machine vision) en medische toepassingen.

Visuele waarneming is het vermogen om licht te detecteren en te interpreteren. In hoofdstuk 1 worden verschillende manieren van visuele waarnemingen in het dierenrijk kort beschreven. Onder de bekende optische vision-systemen in de natuur, is het enkelvoudige oog populairder bij de camera-onderzoekers vanwege het vermogen om beelden van hoge resolutie te creëren. Facet-ogen zijn niet geschikt voor hoge resolutie beelden. Maar ze staan bekend om het efficiënter verwerken van beschikbare informatie en zijn een goed voorbeeld van low power vision systemen. Het licht afkomstig van reflecties is gedeeltelijk gepolariseerd. Een enkelvoudig oog is hiervoor blind, maar facet-ogen zijn in staat om polarisatie te detecteren. De facet-ogen zijn ook meer geschikt voor bewegingsdetectie, hebben een hoge gevoeligheid met weinig licht en bieden een ruim gezichtsveld tegen de kostprijs van een lage optische resolutie. Hoewel facet-ogen niet de beste zijn op het gebied van de beeldkwaliteit, maken de voordelen die zij bieden ze beter geschikt voor machine vision toepassingen dan een enkelvoudig oog.

Facet-ogen zijn samengesteld uit vele ommatidia of facetten. De natuurlijke facet-ogen zijn globaal ingedeeld in appositie en superpositie ogen. 
In appositie ogen ontvangt elk facet slechts licht uit een enkele richting, terwijl in superpositie-ogen de lichtstralen van verschillende facetten wordt gesuperponeerd. In het ontwerp van een kunstmatig facet-oog geïnspireerd door het natuurlijke facet-oog, zijn een micro-array optische lens en een detectorarray nodig. In hoofdstuk 2 wordt een realisatie van een camera systeem gepresenteerd, waarbij de scene optisch verdeeld is over een veelvoud van lenzen, gebruikmakende van één detector-array. Om de scene optisch te verdelen is een samengestelde lensstructuur ontworpen door de Vrije Universiteit Brussel (VUB) binnen een samenwerkingsverband. Het ontworpen objectief heeft een beeldhoek van $124^{\circ}$, een maximaal contrast van 0,3 lijnparen per graad $\left(\mathrm{LP} /{ }^{\circ}\right)$ en een hoek resolutie van minder dan $1^{\circ}$.

In hoofdstuk 2 is het ontwerp van de detector-array voor het gewenste optische systeem gepresenteerd. De ontworpen beeldsensor bestaat uit een raster van 128 bij 128 pixels, beslaat een oppervlakte van $5 \mathrm{~mm}$ x $4 \mathrm{~mm}$, en het is ontworpen en gefabriceerd in het $0.18 \mu \mathrm{m}$ CMOS CIS proces van UMC. De pixel die wordt gebruikt in het ontwerp is een smart pixel, dit is een soort van actieve pixel sensor. Elke pixel bevat een pinned fotodiode $\left(p^{+} / n / p\right)$ en 33 transistors om laag niveau beeldverwerking uit te kunnen voeren. De grootte van de pixel is $25 \mu \mathrm{m} \times 25 \mu \mathrm{m}$ en de grootte van de fotodiode is $10 \mu \mathrm{m} \times 10 \mu \mathrm{m}$, wat overeenkomt met $16 \%$ van de totale oppervlakte. De pixel heeft ook een in-pixel analoge en digitale geheugen, welke in-pixel digitalisering en real-time aanpassingen voor dynamisch bereik mogelijk maken. De ontworpen beeldsensor kan bediend worden in twee modi: een dubbele differentiële modus en een differentiële imaging-modus. In geval van dubbele differentiële modus, wordt de spanning van de fotodiode op elke pixel tweemaal gesampled: één keer na een reset en één keer na de belichtingsperiode. In de differentiële modus worden twee samples verkregen na verschillende belichtingsperioden.

In de ontworpen beeldsensor wordt slechts de helft van het beschikbare pixel array van 128 bij 128 gebruikt voor de normale beeldvorming. De andere helft van het pixel array wordt gebruikt om polarisatie informatie te analyseren met behulp van een metalen draadrooster structuur die besproken wordt in hoofdstuk 3. De drie basiskenmerken van licht zijn intensiteit, kleur en polarisatie. Polarisatie visie en de mechanismen van de polarisatie van licht uit een ongepolariseerde lichtbundel samen met de representatie van de polarisatie gegevens worden ook in detail besproken in hoofdstuk 3. Polarisatie visie is een generalisatie van intensiteit en is analoog aan kleurwaarneming. In paragraaf 3.5 wordt een metalen draadrooster micropolarizer gepresenteerd, die werkzaam is in de ruimtelijke-modus, en is ontworpen met behulp van de metaallagen 
van een standaard CMOS-technologie. Normaalgesproken zou een dergelijke draadrooster structuur fungeren als een eenvoudig diffractierooster, maar wanneer de onderlinge afstand van de draden minder is dan de helft van de golflengte van het invallende licht, wordt het een polarisator. De ontworpen polarisatie sensing sensor heeft een geïntegreerde lineaire polarisator in elke pixel met verschillende oriëntaties. De lineaire draadrooster polarisator is geïmplementeerd met behulp van dunne metalen strips met een lijnbreedte/tussen-ruimte van 240nm/240nm ("pitch" van 480nm). De absorptie van de EM golven om volledige $s$-polarisatie of $p$-polarisatie van de uitgezonden golf te bereiken met behulp van het draad grid is afhankelijk van de pitch van hetgrid. Zodoende varieert de prestatie van het draadrooster als een polarisator ook met de pitch. Met de schaling van de CMOS-technologie zal de pitch van het draadrooster verkleinen, en daardoor verbetert de prestatie als een polarisator.

Het pixel array gebruikt voor polarisatie sensing is verder opgesplitst in twee 64 bij 64 pixels regio's en worden benoemd als sensor-regio 1 en 2 . In sensorregio 1 is het draadrooster horizontaal en verticaal georiënteerd om de $0^{\circ}$ en $90^{\circ}$ polarisatie te meten. In sensor-regio 2 is naast de horizontale en verticale richting ook $45^{\circ}$ oriëntatie van het draadrooster toegepast. Lineaire polarisators worden gekarakteriseerd door twee belangrijke specificaties: de transmissie en de extinctie verhouding. In sensor-regio 1 is de maximaal waargenomen doorlaatbaarheid voor $0^{\circ}$ polarisatie $38,9 \%$, en de minimale doorlaatbaarheid $7 \%$. Voor $90^{\circ}$ polarisatie werden een maximum van $44,4 \%$ en een minimum van $0,1 \%$ waargenomen. Evenzo voor sensor-regio 2 , is de waargenomen doorlaatbaarheid voor $0^{\circ}$ polarisatie maximaal $38,4 \%$ en minimaal $5,4 \%$, terwijl dit voor $90^{\circ}$ polarisatie maximaal $42,2 \%$ en minimaal $0,6 \%$ is. De extinctie factoren verkregen in sensor regio's 1 en 2 zijn respectievelijk 6,3 en 7,7. Deze extinctie factoren zijn hoger dan gemeld in de literatuur voor configuraties die vergelijkbaar zijn met de ontworpen sensor.

In hoofdstuk 4 wordt materiaal classificatie op basis van polarisatie informatie gepresenteerd. Materialen zijn globaal ingedeeld in metalen en diëlektrica. De classificatie van de materiaalsoort kan vitale informatie geven over de omgeving in computer of machine vision. Ongepolariseerd licht wordt gedeeltelijk gepolariseerd na spiegelende reflectie. De status van polarisatie voor de diffuse en spiegelende elementen van de reflectie hangt af van het reflecterende oppervlak, en de meting van de toestand van de polarisatie van het gereflecteerde licht fungeert als een indicator voor het type materiaal oppervlak. De grote van de oscillaties van de maximale en minimale uitgezonden straling ten gevolge van de variaties in het reflectie patroon van het metaal en het diëlektrische 
oppervlak bleken nuttig te zijn in hun classificatie. Van diverse andere gemeten parameters, zoals de mate van polarisatie en de polarisatie Fresnel ratio, werd ook aangetoond dat ze maatgevend zijn voor variaties in het reflectie patroon van de metaal en diëlektrische oppervlakken. De Stokes mate van polarisatie werd ook geëvalueerd om materialen te classificeren. De polarisatie van het gereflecteerde licht varieert ook met de geleidbaarheid van het metalen oppervlak en dit werd verder onderzocht. Het is gebleken dat het mogelijk is dat dit kan dienen als een instrument bij het indelen van hoog en lager geleidende metalen oppervlakken.

In hoofdstuk 5 wordt navigatie met behulp van gepolariseerd licht besproken. De navigatie-strategieën van insecten met natuurlijk-licht (skylight) polarisatie zijn interessant voor toepassingen in de autonome robot navigatie, omdat ze heel weinig informatie nodig hebben. Het polarisatie patroon in natuurlijklicht varieert op een systematische manier, zowel in e-vector en de mate van polarisatie, afhankelijk van de stand van de zon. De Stokes parameters worden gebruikt om de ellipticiteit en azimuth hoeken van een Poincare bol te berekenen en deze hoeken blijken gecorreleerd te zijn met de richting van de inkomende lichtstraal. Een correlatiecoëfficiënt hoger dan 0,94 werd verkregen in alle metingen. Deze methode biedt dus een manier om de richting van inkomend gepolariseerd licht te bepalen die kan worden gebruikt om de hoek positionele informatie te bepalen, die nuttig is voor de autonome robot navigatie. De mogelijkheid om de positie-informatie op de chip te berekenen of in real-time te bepalen kan resulteren in sterk geminiaturiseerde navigatie-sensoren en in het beperken van de rekenkracht. Verder bleek de polarisatie-hoek te variëren met de polarisatie van de richting van de inkomende lichtstraal. Deze informatie kan ook dienen als een kompas voor autonome robot navigatie algoritmes. De variatie in de mate van polarisatie met de positie van de zon kan verder worden onderzocht om de stand van de zon te detecteren op basis van natuurlijk-licht polarisatie.

In hoofdstuk 6 wordt het vermogen van een insect om snel bewegingen te detecteren onderzocht en wordt ook het concept van de weergave van polarisatie informatie in binaire vorm gepresenteerd. Het vermogen van vliegende insecten om snelle bewegingen te detecteren in de visuele omgeving en botsingen te voorkomen met laag niveau beeldverwerking en weinig rekenkracht maakt hun visuele verwerking interessant voor real-time bewegings/botsings detectie in machine vision toepassingen. De ontworpen beeldsensor kan ook worden gebruikt in de temporele differentiële modus en ruimtelijke integratie van eendim-ensionale binaire optische flow om beweging te detecteren/botsing van bewegende objecten repliceren van de "flikkerende effecten"van de insecten. De binaire optische flow wordt gegenereerd in de pixel, gebaseerd op meerdere 
beelden die zijn opgeslagen in het in-pixel geheugen, en ruimtelijk worden geïntegreerd met behulp van een teller. Zowel horizontale als verticale bewegingen kunnen gemakkelijk gedetecteerd worden met behulp van de binaire optische flow die relatief eenvoudig berekend worden in de pixel. Een botsing waarschuwings algoritme voor een naderend object tot $2 \mathrm{~cm}$ afstand is operationeel aangetoond. Dergelijke nauwkeurige botsing waarschuwings algoritmes maken het mogelijk om eenvoudige, geminiaturiseerde en laag-vermogen autonome navigatie robots te ontwerpen. In de differentiële beeldbewerking modus biedt de ontworpen sensor een bewegingsdetectie systeem, dat invariant is voor de achtergrondverlic-hting. Het is aangetoond dat de gegenereerde binaire optische flow een hoekafhankelijk-heid heeft met de lineaire polarisator, en door het verhogen van de draadrooster oriëntaties zal de digitale representatie van de polarisatie de analoge vorm benaderen.

In hoofdstuk 7 wordt, samen met de samenvatting van de resultaten, een meerkanaals beeldverwerkingssysteem besproken. De integratie van de samengestelde lens van de VUB met de ontworpen detector-array kan resulteren in een meerkanaals beeldverwerkingssysteem. Met een breed gezichtsveld en een lage hoek resolutie, zouden deze optische vision systemen niet alleen een toepassing kunnen vinden in machine vision, maar ook in medische applicaties. 


\section{Acknowledgments}

"You can complain because roses have thorns, or you can rejoice because thorns have roses", exactly summarizes how you want to look into the four long years spent on this $\mathrm{PhD}$. It is so very obvious to be influenced by many during the course of these seemingly never ending years.

There are many whom I want to thank for helping me finish with my thesis. I will start with my promoter Albert Theuwissen, who has been very supportive and encouraging over these years. I am really thankful to him for accepting me as a $\mathrm{PhD}$ student even with me not having any background knowledge on image sensors and for being a constant source of motivation.

I would also like to thank Imec, Eindhoven for financing the project and particularly Bert Gyselinckx who offered me the PhD position at Imec and Margot Nijkamp who took care of the official requirements. For the New Year celebrations, I was gifted twice by Holst, the normal Christmas gift and a new supervisor to work with which made life more interesting. I started the project with Guy Meynants and I am immensely grateful to him for imparting to me his knowledge on image sensors. I designed my first working chip under his supervision and it will remain an unforgettable achievement for years to come. Partick Merken taught me to be patient and it was fun to have him around. If my technical writing ability has improved the credit has to go to San Segundo Bello David, for his constant guidance on this aspect. He has been very supportive of my work and has always motivated me. David has also immensely helped me in writing my thesis.

I would also like to thank Chris Van Hoof for all the travel budgets for the conferences that I attended. I imagined Chris declining the idea every time I approached him for a conference attendance but he never did. The exposure that I got from the conferences was really very helpful for my scientific and personal growth, and I am really indebted to him for that. Thank you, Chris.

The office colleagues in Imec, Eindhoven have always supported me in my 
work. I would particularly like to mention a few names, Jiawei with whom I had lots of discussions on amplifiers and general analog circuit design. Besides circuit design we often ended up talking about the share markets, one passion that we both shared and it helped that we were in the same office. Richard van der Hoven, my coffee partner and Friday evening party mate. I always had a company to go out with and that helped to charge up after a long week at work. Thanks to Richard and Pieter Harpe for helping me to translate my summary and propositions to Dutch. All the other office colleagues were also supportive always. Amongst all, I would like to particularly acknowledge Phillips Mattelaer, the Business Development Manager of Holst. Occasionally he would drop by my office and would ask his famous question "Can your facet eye do this?"to which I always replied "Sure, why not". Thank you Phillip for all the discussions we have had on crazy technologies possible using the insect's vision and for always motivating me to think for ideas and possibilities.

I would also like to sincerely thank Adri Mierop of Dalsa for allowing me to use his experimental setup for measurements and helping me with the debugging process that eventually brought the sensor to life. The thesis writing has been a long journey and thanks Mini for helping me with it.

I would also like to thank Hans Boeve and Martin Ouwerkerk from Philips research, Eindhoven who helped me to move to Netherlands from Germany for my master thesis and later was when applying for a $\mathrm{PhD}$ position at Holst. Particularly I want to thank Hans who showed a lot of confidence in me and encouraged me to go for a $\mathrm{PhD}$ in the first instance.

And last but not the least, I would like to thank my family who has been a pillar of support all these years. I particularly thank my Mom who supported me in all my engagements and had confidence in me all the way even when others doubted. Love you, Mom.

Mukul Sarkar

Eindhoven, January 2011 


\section{List of publications}

\section{Journal papers}

M. Sarkar, D. San Segundo, C. Van Hoof and A. J. P. Theuwissen, "Integrated polarization analyzing CMOS image sensor for real time material classification". IEEE Sensors Journal. (Accepted for publication)

M. Sarkar, D. San Segundo, C. Van Hoof and A. J. P. Theuwissen, "Integrated polarization analyzing CMOS for navigation and incoming light ray direction". IEEE Transactions of Instrumentation and Measurement. (Accepted for publication)

\section{Conference papers}

M. Sarkar, D. San Segundo, C. Van Hoof and A. J. P. Theuwissen, "A biologically inspired collision detection algorithm using differential optic flow imaging". Proceedings of Biomedical Circuits and Systems, pp. 250-253, 2010.

M. Sarkar, D. San Segundo, C. Van Hoof and A. J. P. Theuwissen, "Biologically inspired autonomous agent navigation using Stokes parameters and an integrated polarization analyzing CMOS image sensor". Proceedings of Eurosensors XXIV conference, vol. 5, pp. 673676, 2010.

M. Sarkar, D. San Segundo, C. Van Hoof and A. J. P. Theuwissen, "An analog and digital representation of polarization using CMOS image sensor". Proceedings of 
5th European optical society tropical meeting on advanced imaging techniques, ISBN 978-3-00-030503-0, 2010.

M. Sarkar, D. San Segundo, C. Van Hoof and A. J. P. Theuwissen, "Integrated polarization analyzing CMOS image sensor for autonomous agent navigation using polarized light". Proceeding of IEEE International conference on Intelligent Systems, pp. 224-229, 2010.

M. Sarkar, D. San Segundo, C. Van Hoof and A. J. P. Theuwissen, "Integrated polarization analyzing CMOS image sensor". Proceeding of IEEE International Symposium on circuits and systems, pp. 621-624, 2010.

E. Moens, Y. Meuret, H. Ottevaere, M. Sarkar, D. San Segundo, P. Merken and H. Thienpont, "Design of a miniaturized imaging system with a very large field of view". Proceeding of SPIE, vol. 7716, 77162D, 2010.

M. Sarkar, D. San Segundo, C. Van Hoof and A. J. P. Theuwissen, "Integrated polarization analyzing CMOS image sensor for detecting incoming light ray direction". Proceeding of IEEE Sensors Application Symposium, pp. 194-199, 2010. 


\section{About the author}

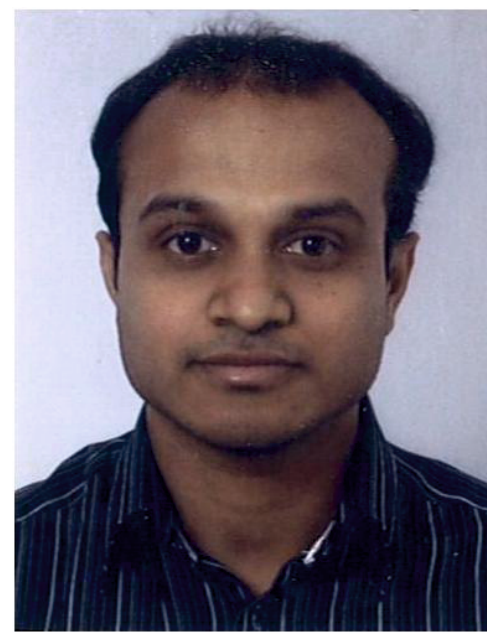

Mukul Sarkar received his B.E. degree in Electronic and Instrumentation from Andhra University, Visakhapatnam, India in 2002 and MSc. degree in Biomedical Engineering from University of Technology, Aachen, Germany in 2006. The title of his MSc. thesis was "Sensor module architecture for small autonomous networked devices".In 2007 he started to work towards $\mathrm{PhD}$ degree in the electronics instrumentation laboratory, Faculty of EEMCS, Delft, University of Technology (TU Delft)with Prof. A.J.P. Theuwissen on the subject of biologically inspired CMOS image sensors.

From December 2003 till May 2005, he continued to work in the Philips Institute of medical information, Helmholtz institute, Aachen, Germany as a student research assistant mainly in the field of detection and analysis of biosignals. From September 2005 to March 2006, he took an internship with Philips research, Eindhoven, Netherlands working on digital algorithm implementation on small asynchronous networked decides. His professional interest includes research on biomedical instrumentation and bio-inspired vision sensors. 


